\title{
Artificial gauge fields with ultracold atoms in optical lattices
}

Monika Aidelsburger

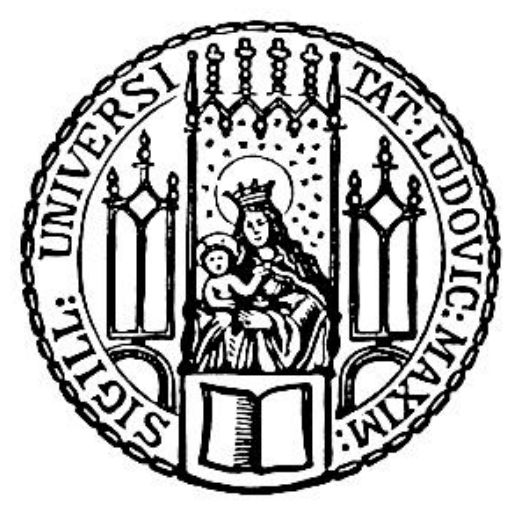

München 2015 



\section{Artificial gauge fields with ultracold atoms in optical lattices}

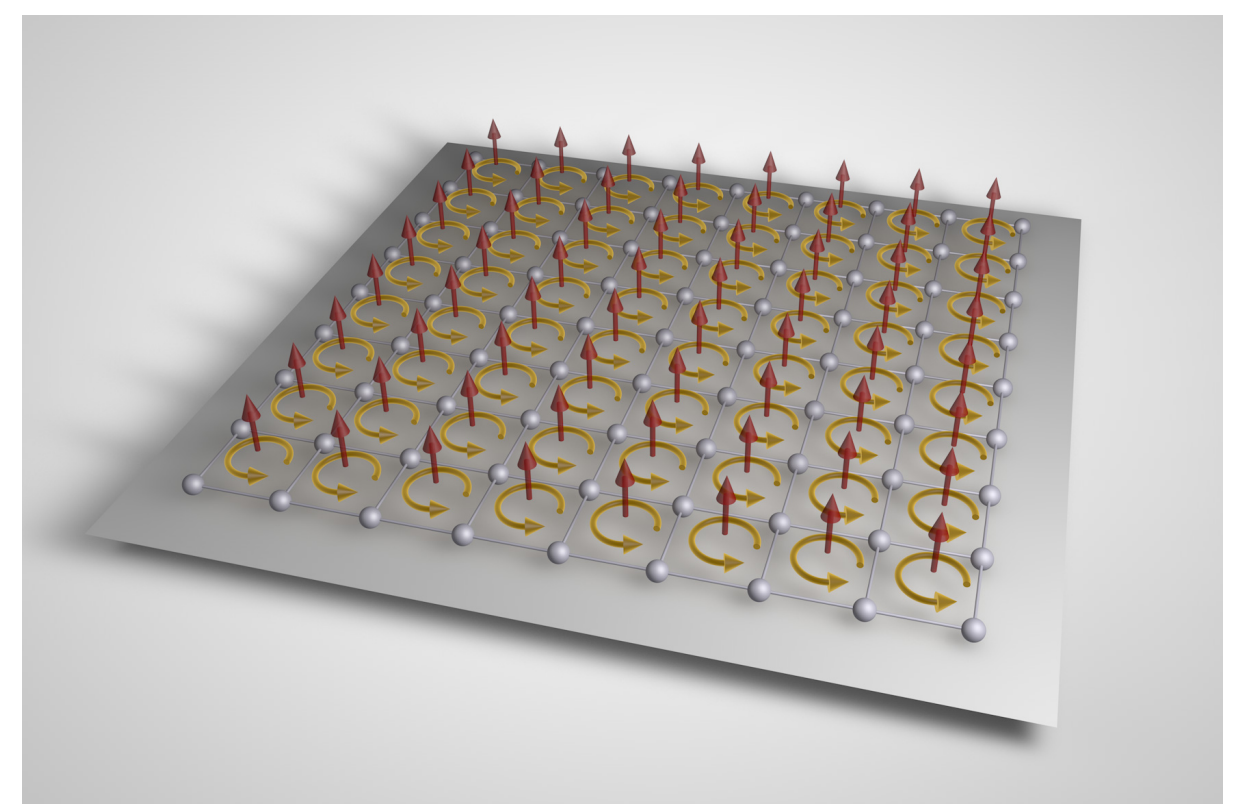

Dissertation der Fakultät für Physik

der Ludwig-Maximilians-Universität München

vorgelegt von

Monika Aidelsburger

geboren in Aichach

München, 19. Dezember 2014 
Tag der mündlichen Prüfung: 4. Februar 2015

Erstgutachter: Prof. I. Bloch

Zweitgutachter: Prof. J. Dalibard

Weitere Prüfungskommissionsmitglieder: Prof. J. von Delft, Prof. W. Zinth 


\section{Zusammenfassung}

Diese Doktorarbeit befasst sich mit der Erzeugung von künstlichen Magnetfeldern für ultrakalte Atome in optischen Gittern mithilfe von Laser-induziertem Tunneln sowie mit der ersten experimentellen Bestimmung der Chernzahl in einem nicht-elektronischen System.

Kalte Atome in optischen Gittern lassen sich experimentell sehr gut kontrollieren, was sie zu guten Modellsystemen für die Simulation von Festkörpern macht, wobei die Atome die Rolle der Elektronen übernehmen. Allerdings können Magnetfeldeffekte in diesen Systemen nicht direkt im Experiment simuliert werden, da die Atome elektrisch neutral sind, weshalb auf sie keine Lorentzkraft wirkt. Im Rahmen dieser Doktorarbeit wird eine neue Methode vorgestellt künstliche Magnetfelder basierend auf Laser-induziertem Tunneln zu erzeugen um somit die Physik geladener Teilchen in realen Magnetfeldern nachzuahmen. Dabei verursachen Laserstrahlen eine periodische Modulation der einzelnen Gitterplätze, deren Phase von der Gitterposition abhängt und dadurch zu komplexen Tunnelkopplungen führt. Ein Atom, welches sich entlang einer geschlossenen Bahn in diesem System bewegt, erfährt eine Phase, die als Aharonov-Bohm-Phase eines geladenen Teilchens in einem Magnetfeld interpretiert werden kann. Das modulierte Gitter wird durch einen zeitabhängigen Hamilton-Operator beschrieben, der typischerweise durch einen effektiven zeitunabhängigen Floquet Hamilton-Operator genähert wird. Im Rahmen dieser Arbeit wird darüber hinaus die vollständige Zeitabhängigkeit innerhalb einer Modulationsperiode beschrieben und mit den experimentellen Daten verglichen. Mithilfe des Laser-induzierten Tunnelns wurden alternierende sowie gleichgerichtete Magnetfelder im Experiment erzeugt, wobei letztere eine Realisierung des Harper-Hofstadter-Modells für einen Fluss $\Phi=\pi / 2$ pro Gittereinheitszelle darstellen. Durch die Verwendung eines zusätzlichen PseudospinFreiheitsgrades konnte zudem der Spin-Hall-Effekt in einem optischen Gitter beobachtet werden. Unter Benutzung der einzigartigen Detektions- und Manipulationstechniken eines zweidimensionalen Übergitters konnte die Stärke und Verteilung des künstlichen Magnetfeldes auf lokaler Ebene durch die Beobachtung von Zyklotronorbits experimentell bestimmt werden. Die Bandstruktur in einem periodischen Potential mit externem Magnetfeld weist interessante topologische Eigenschafen auf, die durch Chernzahlen beschrieben werden, welche beispielsweise dem Quanten-Hall-Effekt zugrunde liegen. Um topologische Bandeigenschaften mit kalten Atomen beobachten zu können, wurden die genannten experimentellen Techniken weiterentwickelt. Mit einem neuen Aufbau, der nur auf optischen Potentialen beruht, konnte erstmals die Chernzahl in einem nicht-elektronischen System bestimmt werden.

Die vorgestellten experimentellen Methoden eröffnen einzigartige Möglichkeiten die Eigenschaften von topologischen Materialien mit kalten Atomen in optischen Gittern zu untersuchen. Die Techniken wurden mit bosonischen Atomen implementiert, sie lassen sich allerdings ohne weiteres auch auf fermionische Systeme anwenden. 


\section{Abstract}

This thesis reports on the generation of artificial magnetic fields with ultracold atoms in optical lattice potentials using laser-assisted tunneling, as well as on the first Chernnumber measurement in a non-electronic system.

The high experimental controllability of cold atoms in optical lattices makes them suitable candidates to study condensed matter Hamiltonians, where the atoms play the role of the electrons. However, the observation of magnetic field effects in these systems is challenging because the atoms are charge neutral and do not experience a Lorentz force. In the context of this thesis a new experimental technique for the generation of effective magnetic fields with laser-assisted tunneling was demonstrated, which mimics the physics of charged particles in real magnetic fields. The applied laser beams create a periodic on-site modulation whose phase depends on the position in the lattice and leads to complex tunnel couplings. An atom that hops around a closed loop in this system picks up a non-zero phase, which is reminiscent of the Aharonov-Bohm phase acquired by a charged particle in a magnetic field. The corresponding time-dependent Hamiltonian is typically described in terms of an effective time-independent Floquet Hamiltonian. In this work a theoretical description of the underlying full-time dynamics that occurs within one driving period and goes beyond the simple time-independent picture is presented. In the experiment the laser-assisted-tunneling method was implemented for staggered as well as uniform flux distributions, where the latter is a realization of the Harper-Hofstadter model for a flux $\Phi=\pi / 2$ per lattice unit cell. By exploiting an additional pseudo-spin degree of freedom the same experimental setup led to the observation of the spin Hall effect in an optical lattice. Using the unique experimental detection and manipulation techniques offered by a two-dimensional bichromatic superlattice potential the strength of the artificial magnetic field and its spatial distribution could be determined through the observation of quantum cyclotron orbits on the level of isolated four-site square plaquettes. The band structure in the presence of a uniform magnetic field is topologically non-trivial and is characterized by the Chern number, a 2D topological invariant, which is at the origin of the quantized Hall conductance observed in electronic systems. In order to probe the topology of the bands the techniques mentioned above were refined by developing a new all-optical laser-assisted tunneling setup, which enabled the first experimental determination of the Chern number in a non-electronic system.

The presented measurements and techniques offer a unique setting to study the properties of topological systems with ultracold atoms. All experimental techniques that were developed in the context of this thesis with bosonic atoms can be directly applied to fermionic systems. 


\section{Contents}

1 Introduction 1

2 Square lattice with magnetic field $\quad 7$

2.1 Peierls phase-factors . . . . . . . . . . . . . . 8

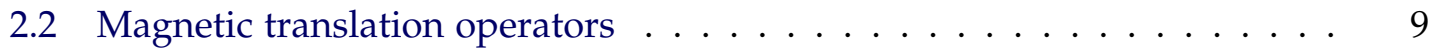

2.2.1 Homogeneous magnetic fields . . . . . . . . . . . . . . . . . 11

2.2.2 Magnetic translation operators for $\alpha=1 / 4 \ldots \ldots \ldots 13$

2.3 Harper-Hofstadter Hamiltonian ．.. . . . . . . . . . . . . . . . 15

2.3.1 Single particle energy spectrum . . . . . . . . . . . . . . . . . . . . . . . . 15

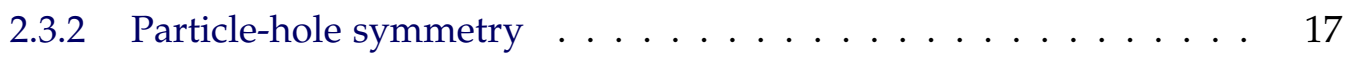

2.4 Chern number . . . . . . . . . . . . . . . . . . . . . . . . . . . 19

2.4.1 Distribution in the Hofstadter model . . . . . . . . . . . . 20

2.4 .2 Diophantine equation . . . . . . . . . . . . 20

2.4.3 Numerical calculation of the Chern number . . . . . . . . . . . 21

3 Artificial gauge fields with laser-assisted tunneling 23

3.1 Periodically driven quantum systems . . . . . . . . . . . . . . . . 24

3.1.1 Floquet formalism . . . . . . . . . . . . . . . . . . 24

3.1.2 Mapping to time-independent Hamiltonian . . . . . . . . . . . 27

3.1.3 Magnus-expansion approach . . . . . . . . . . . . . . 30

3.1.4 Comparison of the two methods: Two-level system . . . . . . . . . 31

3.2 Peierls phases and multi-photon processes . . . . . . . . . . . . . 34

3.3 Periodically driven one-dimensional lattices . . . . . . . . . . . . . . 35

3.3.1 Micro-motion staggered superlattice potential . . . . . . . . . . . . . 36

3.3.2 Micro-motion Wannier-Stark ladder . . . . . . . . . . . . . . . . 38

3.4 Extension to two dimensions: artificial gauge fields . . . . . . . . . . . 39

4 Overview of the experimental setup and measurement techniques 43

4.1 Towards quantum degeneracy . . . . . . . . . . . . . . . . . 44

4.2 Optical lattice potentials . . . . . . . . . . . . . . . . 47

4.3 Superlattice potential . . . . . . . . . . . . . . . . . . . . . 48

4.3 .1 Phase calibration . . . . . . . . . . . . . . . . . 49 
4.3.2 Calibration of the staggered superlattice potential . . . . . . . . . 51

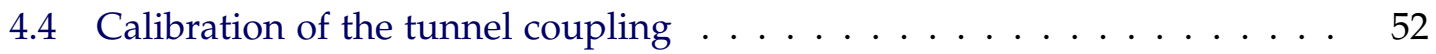

4.5 Filtering sequence . . . . . . . . . . . . . . . . 54

4.6 Site-resolved detection $\ldots \ldots \ldots \ldots \ldots \ldots$

5 Staggered magnetic flux $\quad 59$

5.1 Single-particle Hamiltonian ．.. . . . . . . . . . . . . . . . . . . . . . . 60

5.2 Gauge-dependence of expansion images . . . . . . . . . . . . . . 63

5.3 Experimental setup . . . . . . . . . . . . . . . . 65

5.4 Effective Floquet Hamiltonian and micro-motion $\ldots \ldots \ldots 7$

5.5 Ground state of the staggered flux lattice . . . . . . . . . . . . . 71

5.5 .1 Isotropic coupling $J=K \ldots \ldots \ldots \ldots \ldots \ldots \ldots$

5.5 .2 Dependence on the coupling ratio $J / K \ldots \ldots$. . . . . . 75

5.5 .3 Micro-motion in the staggered flux lattice . . . . . . . . . . 78

5.6 Local probe of the artificial gauge field . . . . . . . . . . . . . . 79

5.6 .1 Phase evolution . . . . . . . . . . . . . . 80

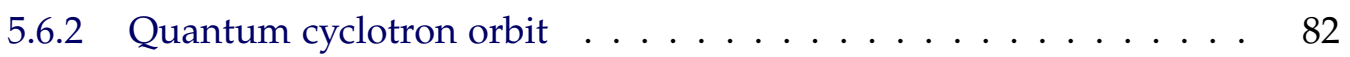

5.7 Full-time dynamics of the cyclotron orbits . . . . . . . . . . . . . . 84

5.7 .1 Off-resonant driving . . . . . . . . . . . . . 85

5.7 .2 Initial kick-operator and micro-motion $\ldots \ldots \ldots$. . . . . . 87

6 Harper-Hofstadter model and spin Hall effect 93

6.1 Experimental setup . . . . . . . . . . . . . . . . . . . 94

6.1 .1 Harper-Hofstadter model . . . . . . . . . . . . . . . . . . . . 94

6.1.2 Spin-dependent Harper-Hofstadter model . . . . . . . . . . . . 96

6.2 Laser-assisted tunneling in a tilted lattice potential . . . . . . . . . . . 97

6.3 Probing the magnetic flux distribution . . . . . . . . . . . . . . . 99

6.3.1 Experimental sequence . . . . . . . . . . . . . . . 102

6.3 .2 Numerical simulations . . . . . . . . . . . . . . . . . . . . 103

6.4 Spin Hall effect in an optical lattice . . . . . . . . . . . . . . . . 106

$\begin{array}{lll}7 & \text { All-optical setup for flux rectification } & 109\end{array}$

7.1 Laser-assisted tunneling on every other bond . . . . . . . . . . . . . . 110

7.2 Uniform flux in a staggered potential . . . . . . . . . . . . . . . . . 112

7.3 Experimental setup . . . . . . . . . . . . . . . . . . 116

7.3.1 Intensity modulation . . . . . . . . . . . . . . . . . 118

7.3.2 Controlling the relative phase of the modulation . . . . . . . . 121

7.4 Staggered flux distribution $\ldots \ldots \ldots \ldots . \ldots \ldots$ 
8 Chern-number measurement of Hofstadter bands $\quad \mathbf{1 2 5}$

8.1 Hofstadter model for a flux $\Phi=\pi / 2 \ldots \ldots$. . . . . . . . . 126

8.2 Anomalous Hall velocity and Chern-number measurement . . . . . . . . 128

8.3 Adiabatic loading into the Hofstadter bands . . . . . . . . . . . . . . . . 131

8.3.1 Loading sequence: general idea . . . . . . . . . . . . . . . . . 131

8.3.2 Experimental sequence . . . . . . . . . . . . . . 133

8.3.3 Momentum distribution and initial band population . . . . . . . . 133

8.4 Transport measurements . . . . . . . . . . . . . . . . . . . . 135

8.4.1 Short-time dynamics . . . . . . . . . . . . . . . . 136

8.4.2 Long-time dynamics and band populations . . . . . . . . . . . 138

8.4.3 Characterization of the topological phase transition . . . . . . . 143

9 Conclusions and Outlook $\quad 147$

$\begin{array}{ll}\text { A Magnetic translation operators } & 151\end{array}$

$\begin{array}{ll}\text { B Propagation operator } & 153\end{array}$

$\begin{array}{ll}\text { C Staggered flux distribution } & 155\end{array}$

$\begin{array}{ll}\text { References } & 157\end{array}$ 


\section{Chapter 1}

\section{Introduction}

Quantum states of matter have been successfully characterized by the concept of spontaneous symmetry breaking [1]. A descriptive example is the transition from a paramagnetic to a ferromagnetic state of a metal. Above a critical temperature, known as the Curie temperature, the direction of the spins in the material is random and the average magnetization $\langle\mathbf{M}\rangle$ is zero. Below a critical temperature the system enters a ferromagnetic phase, where the spins are at least partially aligned, such that the magnetization $\langle\mathbf{M}\rangle$ takes a finite value. The ferromagnetic state is no longer invariant under spin rotation; an additional symmetry is broken compared to the paramagnetic state. The transition is characterized by the order parameter $\langle\mathbf{M}\rangle$, which is zero for the symmetric state and non-zero for the symmetry-breaking state.

With the discovery of the integer quantum Hall $(\mathrm{QH})$ effect $[2,3]$ a new family of quantum states was found which did not fit into this simple classification of condensed matter systems described above [4-6]. In the QH effect electrons form a $\mathrm{QH}$ insulator, that is insulating in the bulk but displays current-carrying states at its boundaries. Each of these states contributes one quantum of conductance $e^{2} / h$, which gives rise to the perfectly quantized Hall conductance discovered in 1980 [2, 3]. Surprisingly, this quantization was found independent of the microscopic details of the material $[7,8]$. In fact the number of edge states is mathematically determined by the value of an integer topological invariant such that the quantization is topologically protected against small perturbations of the system.

In mathematics the term topology was introduced by Johann Benedict Listing in the 19th century [9] to classify the shape of geometric objects. He started to establish a new language to describe what one could call the qualitative shape of a geometric object without making use of ordinary quantities that are usually employed to define the shape of an object. Intuitively one would say that a sphere is intrinsically different from a torus because they cannot be continuously transformed into each other. The torus belongs to a class of objects with genus $g=1$, that are characterized by a hole independent of the specific details of its shape. In contrast a sphere belongs to a class with genus $g=0$ 
and does not exhibit a hole. In this sense a coffee cup is topologically equivalent to a torus and the genus is a topological invariant. It was found that mathematically these numbers can be described as integrals over the local curvature of a surface. Even though the integrands locally depend on the details of the geometry, the integral itself however does not.

In condensed matter systems the abstract concept of topology can be applied to determine the intrinsic properties of the corresponding energy bands. Two systems with a gapped energy spectrum can only be continuously transformed into each other if they belong to the same topological class. Otherwise the system undergoes a quantum phase transition during the transformation where the energy spectrum becomes gapless. Accordingly, smooth deformations can be defined as transformations that do not close an energy gap [4,6]. It turns out that observables such as the quantized conductivity in the integer $\mathrm{QH}$ effect, that arise due to the topological properties of the edge states, are rooted in the topological properties of the bulk [10-12], which are described by the Chern number, a topological invariant defined as an integral in momentum space [8].

Since the discovery of $\mathrm{QH}$ insulators the field of topological materials has been rapidly growing $[4,6,13]$. Initially, it was believed that topological quantum states can only exist in 2D and if time-reversal (TR) symmetry is broken by applying a magnetic field. In 1988 Haldane [14] proposed a model Hamiltonian that supports QH states on a honeycomb lattice without external magnetic field. Such insulators are generally known as Chern insulators and even though these insulators are conceptually different from QH systems they belong to the same class. A generalization of Haldane's model was put forward by Kane \& Mele [15] and Bernevig \& Zhang [16], who predicted TR-symmetric topological insulators due to spin-orbit interaction. In 2D topological insulators are synonymously called quantum spin Hall (QSH) insulators and were observed experimentally in $\mathrm{HgTe} / \mathrm{CdTe}$ quantum well structures [17-19]. QSH materials are closely related to $\mathrm{QH}$ insulators; they are characterized by a charge excitation gap in the $2 \mathrm{D}$ bulk and gapless helical edge states that lie in the bulk gap and are protected by TR symmetry. The edge states appear in pairs at the surface and their propagation direction depends on the spin of the particle. Soon after also topological insulators in $3 \mathrm{D}$ were predicted [20-22] and observed in experiments [23, 24].

The origin of topological insulators can be understood in a single-particle framework. However, the physics becomes even richer in the presence of interactions as was first demonstrated with the discovery of the fractional quantum Hall $(\mathrm{FQH})$ effect $[25,26]$. In addition to the integer $\mathrm{QH}$ plateaus there exist plateaus at fractional values of $e^{2} / h$, which cannot be understood in a single-particle picture. FQH states are particularly interesting because they can feature fractionally-charged excitations which obey unusual statistics that are neither fermionic nor bosonic. In the laboratories $\mathrm{FQH}$ states only exist under extreme conditions; very low temperatures and strong magnetic fields. Similar states are predicted at zero magnetic fields in fractional Chern insulators (FQI), which potentially exist even up to room temperatures $[13,27]$. So far related experimental 
observations have not been reported, however, very recently a Chern insulator without external magnetic field has been observed experimentally [28].

\section{Ultracold atoms in optical lattices}

Synthetic materials that consist of ultracold atoms in optical lattices have proven to be very well suited to simulate and study condensed matter Hamiltonians [29, 30]. Neutral atoms are routinely cooled and trapped in periodic potentials created by interfering laser beams [31]. Using these techniques various lattice geometries can be engineered with a high degree of experimental control [32-37]. The physics of interacting atoms in such periodic potentials can be described by the Hubbard model [38], which is an essential model Hamiltonian to describe strongly-correlated electrons in condensed matter systems. The characteristic parameters are the tunnel coupling between neighboring sites and the on-site interaction [39-41]. For bosonic atoms with repulsive interactions the system undergoes a quantum phase transition at a critical ratio of interaction to kinetic energy [42, 43], which has been observed experimentally in 2001 [44]. With ultracold atoms in optical lattices the Hubbard parameters can be tuned individually in a very clean, defect-free realization by changing the lattice depth, which changes the kinetic energy, or by controlling the interaction strength via Feshbach resonances [45].

Due to the high experimental controllability, these systems constitute promising candidates to gain deeper insight into the rich physics of topological materials. In particular they may provide access to physical observables typically not attainable in solid-state experiments [29]. Ultracold quantum gases are commonly probed after time-of-flight to obtain information about their momentum distribution and coherence properties [29]. New generation experiments have increased the number of accessible observables to a great extent through high-resolution detection techniques [46-51]. Single-site and singleatom resolved density distributions of atoms in optical lattices have become available $[49,50]$. This new imaging techniques provide access to complex spatial correlations [52] and enabled single-site spin control of individual atoms in the lattice [53].

Cold-atom setups might further allow the generation of synthetic topological matter without any counterpart in nature. The most recent example is the implementation of the Haldane model [14] which was considered unlikely to be realized in condensedmatter systems but was demonstrated in a cold-atom setup [54] by breaking TR symmetry in a honeycomb lattice with circular modulation of the lattice position [55].

Typically QH states are associated with 2D electron gases confined in a two-dimensional crystalline potential and subjected to a very large magnetic field. In cold-atom setups related physical effects cannot be observed directly because the charge neutrality of the atoms prevents a direct application of the Lorentz force. To overcome this limitation several experimental techniques have been developed to engineer artificial magnetic fields which mimic the effect of real magnetic fields and many of them were already successfully demonstrated experimentally [56-59]. However, the realization of very strong 
synthetic magnetic fields has so far remained out of reach. It has been shown that atoms in optical lattices might be well suited to enter this regime by coupling their motional and internal degrees of freedom [60-62]. In this thesis, a similar method is discussed, where artificial gauge fields are implemented in optical lattices using laser-assisted tunneling. This scheme has the advantage that it does not rely on the internal structure of the atom but makes use of periodic on-site modulation of the lattice using far-detuned running-wave beams [63-66]. Therefore it is less susceptible to heating due to spontaneous emission. This has lead to the successful implementation of staggered [67-69] as well as uniform flux distributions [70-73] in the strong-field regime and enabled the first experimental observation of a $2 \mathrm{D}$ topological invariant, the Chern number, in a cold-atom setup [73].

\section{Contents of this thesis}

The energy spectrum of an electron moving in a periodic potential is altered due to the presence of a magnetic field in a rather dramatic way even in a single-particle picture [74-76]. Due to the magnetic field the discrete translational symmetry of the Hamiltonian is no longer determined by the symmetry of the underlying crystal structure but by the magnetic translation symmetries which are related to the strength of the magnetic field. A theoretical discussion of the properties of the single-particle Hamiltonian of a square lattice in the presence of an external magnetic field is presented in chapter 2. Amongst others, synthetic magnetic fields can be engineered with laser-assisted tunneling in cold-atom setups. The corresponding time-periodic Hamiltonian can be described using the Floquet theorem, which is similar to Bloch's theorem for spatially periodic Hamiltonians. In chapter 3 the theoretical background of time-periodic Hamiltonians and the generation of artificial gauge fields with laser-assisted tunneling is introduced. All measurements presented in this thesis were performed with bosonic atoms. Each experimental cycle started with the preparation of a Bose-Einstein condensate (BEC) of Rubidium atoms $\left({ }^{87} \mathrm{Rb}\right)$, which was then loaded into several optical lattice and superlattice potentials. A description of the main underlying experimental setup can be found in chapter 4 .

The first successful implementations of artificial gauge fields in 2D optical lattices were staggered flux distributions in triangular [67] and square lattices [68, 69]. In these configurations the direction of the field alternates in sign across the lattice and exhibits a zero mean value. An experimental realization of such a staggered flux distribution with laser-assisted tunneling in a staggered superlattice potential is discussed in chapter 5. The corresponding setup was modified in order to generate uniform flux configurations $[70,71]$ by replacing the staggered potential with a linear one (chapter 6). This constitutes a realization of the Harper-Hofstadter Hamiltonian [74-76] which describes charged particles on a square lattice with uniform magnetic field; it breaks TR symmetry and gives rise to topologically non-trivial bands. Moreover, using the exact same exper- 
imental setup a Hamiltonian [77] underlying the QSH effect $[15,16]$ was implemented by making use of an additional pseudo-spin degree of freedom. This further led to the observation of the spin Hall effect in an optical lattice (chapter 6).

The previously mentioned scheme, which is discussed in chapter 6, relies on a magnetic field gradient and did not allow for an observation of the dynamics in the fully connected two-dimensional lattice but only on a local scale. The main limitation was to find an adiabatic way to connect the topologically trivial 2D lattice without flux to the topologically non-trivial one which exhibits a completely different energy spectrum. In chapter 7 a new all-optical setup is introduced, which generates an effective uniform magnetic field without the requirement of a magnetic field gradient. This greatly increases the experimental flexibility and enabled an adiabatic loading of the atoms into the topologically non-trivial energy bands.

It further provided the basis for the first measurement of a 2D topological invariant in a cold-atom setup [73]. Due to the non-trivial topology of the energy bands the atoms experience a transverse motion as a response to an applied force, which is proportional to the Chern number. The experimental results are reported in chapter 8 .

\section{Publications}

The following articles have been published in the context of this thesis. The articles most relevant for this thesis are marked in bold font.

- Y.-A. Chen, S. Nascimbène, M. Aidelsburger, M. Atala, S. Trotzky, I. Bloch, Controlling Correlated Tunneling and Superexchange Interactions with ac-Driven Optical Lattices, Phys. Rev. Lett. 107, 210405 (2011).

- M. Aidelsburger, M. Atala, S. Nascimbène, S. Trotzky, Y.-A. Chen, I. Bloch, Experimental realization of strong effective magnetic fields in an optical lattice, Phys. Rev. Lett. 107, 255301 (2011).

- S. Nascimbène, Y.-A. Chen, M. Atala, M. Aidelsburger, S. Trotzky, B. Paredes, I. Bloch, Experimental realization of plaquette resonating valence-bond states with ultracold atoms in optical superlattices, Phys. Rev. Lett. 108, 205301 (2012).

- M. Aidelsburger, M. Atala, S. Nascimbène, S. Trotzky, Y.-A. Chen, I. Bloch, $E x$ perimental realization of strong effective magnetic fields in optical superlattice potentials, Invited article Appl. Phys. B 113, 1 (2013).

- M. Atala*, M. Aidelsburger*, J. T. Barreiro, D. Abanin, T. Kitagawa, E. Demler, I. Bloch, Direct measurement of the Zak phase in topological Bloch bands, Nature Physics 9, 795-800 (2013).

* these authors contributed equally to this work 
- M. Aidelsburger, M. Atala, M. Lohse, J. T. Barreiro, B. Paredes, I. Bloch, Realization of the Hofstadter Hamiltonian with ultracold atoms in optical lattices, Phys. Rev. Lett. 111, 185301 (2013).

- M. Atala, M. Aidelsburger, M. Lohse, J. T. Barreiro, B. Paredes, I. Bloch, Observation of chiral currents with ultracold atoms in bosonic ladders, Nature physics 10, 588-593 (2014).

- M. Aidelsburger, M. Lohse, C. Schweizer, M. Atala, J. T. Barreiro, S. Nascimbène, N. R. Cooper, I. Bloch, N. Goldman, Measuring the Chern number of Hofstadter bands with ultracold bosonic atoms, Nature Physics 11, 162-166 (2015).

- N. Goldman, J. Dalibard, M. Aidelsburger, N. R. Cooper, Periodically-driven quantum matter: the case of resonant modulations, Phys. Rev. A 91, 033632 (2015). 


\section{Chapter 2}

\section{Square lattice with magnetic field}

Electrons moving in a periodic potential experience a quantized energy spectrum, where the discrete energy bands are known as Bloch bands. In a magnetic field the spectrum further splits into highly degenerate Landau levels. The interplay between both effects leads to a complex fractal energy spectrum known as Hofstadter's butterfly [76]. In order to observe related effects experimentally, magnetic fields on the order of one flux quantum per lattice unit cell are required. In solid state setups the lattice constants are rather small, i.e. on the order of a few angstroms. Consequently, unfeasible large magnetic fields would need to be applied to the material to enter this regime. To overcome this limitation artificial materials with larger lattice constants can be designed. Recently this was demonstrated by engineering superlattice structures with graphene placed on hexagonal boron nitride [78-80]. Additionally the same experimental regimes became accessible in photonics [81, 82] and with ultracold atoms [70, 71].

Already the single particle physics in a periodic potential with large magnetic field shows very interesting phenomena. The motion of a charged particle in a magnetic field is accompanied by a geometric phase, the Aharonov-Bohm phase [83]. On a lattice these phases are introduced in the form of so-called Peierls phases that a particle picks up when hopping in the lattice (Sect. 2.1). Unlike the zero-field case the magnetic Hamiltonian is not invariant under the usual translation by a lattice unit vector. Instead one has to consider the magnetic translation symmetries of the Hamiltonian which effectively enlarge the usual lattice unit cell depending on the magnetic flux. The new unit cell is denoted as magnetic unit cell. Its area is determined by the strength of the flux but its dimensions are not unique (Sect. 2.2). The resulting single-particle energy spectrum shows a fractal structure as a function of the magnetic flux per unit cell, which is known as Hofstadter's butterfly (Sect. 2.3). Depending on the flux the lowest tight-binding band splits into several subbands, whose topological properties are characterized by topological invariants called Chern numbers, which are directly related to the quantization of the Hall conductivity in the integer QH effect (Sect. 2.4). 


\subsection{Peierls phase-factors}

The physics of electrons moving in a periodic potential can be described by the Hubbard model, which was first introduced by John Hubbard in 1963 [38]. This model is a good approximation for systems at low temperatures where all particles occupy the lowest energy band $[84,85]$. It is typically characterized by two terms: a kinetic term that describes the hopping of particles between neighboring sites in the potential and an on-site interaction term. For a single electron in a 2D lattice potential the Hamiltonian consists only of the kinetic term and can be written in the following form

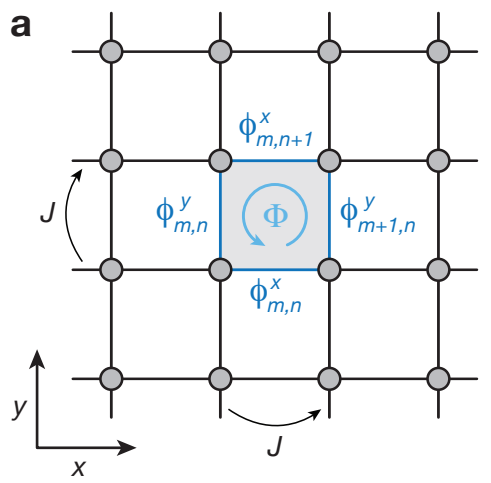

b

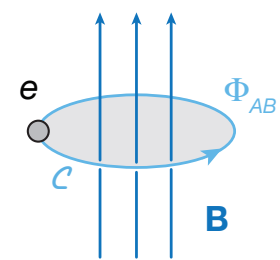

Figure 2.1: Equivalence between complex tunneling amplitudes on a square lattice and the Aharonov-Bohm phase. a Schematic drawing of a 2D lattice with complex tunneling amplitudes determined by the Peierls phases $\phi_{m, n}^{i}, i=\{x, y\}$. The coupling strength along both directions is given by $J$. An electron that tunnels around the borders of one lattice unit cell (gray shaded area) picks up a phase $\Phi=\phi_{m, n}^{x}+\phi_{m+1, n}^{y}-\phi_{m, n+1}^{x}-\phi_{m, n}^{y}$ due to the presence of the vector potential A. $\mathbf{b}$ Illustration of an electron moving along a closed path $\mathcal{C}$ in an external magnetic field $\mathbf{B}=\nabla \times \mathbf{A}$. The particle picks up a geometric phase $\Phi_{A B}$ known as Aharonov-Bohm phase.

$$
\hat{H}_{0}=-J \sum_{m, n}\left(\hat{a}_{m+1, n}^{+} \hat{a}_{m, n}+\hat{a}_{m, n+1}^{\dagger} \hat{a}_{m, n}+\text { h.c. }\right)
$$

where $\hat{a}_{m, n}^{+}$and $\hat{a}_{m, n}$ are the creation and annihilation operators on site $(m, n)$ respectively, $m$ is the site index along $x$ and $n$ the one along $y$. The model is based on the tight-binding approximation where the electrons are assumed to occupy the standard orbitals of the atoms and the overlap between atomic wave functions on neighboring sites is small. The tunneling amplitude for an electron to hop from one atom to the next is determined by the coupling matrix element $J$.

In the presence of an external magnetic field $\mathbf{B}=\nabla \times \mathbf{A}$, where $\mathbf{A}$ is the vector potential, Hamiltonian (2.1) is modified according to the Peierls substitution [86]. As a result the tunneling matrix elements become complex and hopping in the lattice is accompanied by a phase $\phi_{m, n}^{i}=-e A_{m, n}^{i} / \hbar, i=\{x, y\}$, which is known as Peierls phase (Fig. 2.1a), $e$ 
is the electron charge and $\hbar=h /(2 \pi)$ the reduced Planck constant. Accordingly, the tight-binding Hamiltonian takes the following form

$$
\hat{H}=-J \sum_{m, n}\left(\mathrm{e}^{i \phi_{m, n}^{x}} \hat{a}_{m+1, n}^{\dagger} \hat{a}_{m, n}+\mathrm{e}^{i \phi_{m, n}^{y}} \hat{a}_{m, n+1}^{\dagger} \hat{a}_{m, n}+\text { h.c. }\right) .
$$

The Peierls phases are a manifestation of the Aharonov-Bohm phase experienced by a charged particle moving in a magnetic field (Fig. 2.1b)

$$
\Phi_{A B}=-\frac{e}{\hbar} \oint_{\mathcal{C}} \mathbf{A} \cdot \mathrm{d} \mathbf{r}=-2 \pi \Phi_{B} / \Phi_{0}
$$

where $\Phi_{B}$ is the magnetic flux through the area enclosed by the contour $\mathcal{C}$ and $\Phi_{0}=h / e$ is the magnetic flux quantum [83]. Equivalently one can define the magnetic flux per lattice unit cell in units of the magnetic flux quantum as

$$
\alpha=\frac{1}{2 \pi} \Phi=\frac{1}{2 \pi}\left(\phi_{m, n}^{x}+\phi_{m+1, n}^{y}-\phi_{m, n+1}^{x}-\phi_{m, n}^{y}\right) .
$$

In the following $\Phi$ will be denoted as the flux per unit cell of the underlying lattice or simply the flux per plaquette.

\subsection{Magnetic translation operators}

In the zero-field case the lattice translation operators $\hat{T}_{i}^{0}$ commute with Hamiltonian (2.1) for all Bravais lattice vectors [84, 85],

$$
\hat{T}_{x}^{0}=\sum_{m, n} \hat{a}_{m+1, n}^{\dagger} \hat{a}_{m, n}, \quad \hat{T}_{y}^{0}=\sum_{m, n} \hat{a}_{m, n+1}^{\dagger} \hat{a}_{m, n} ;
$$

they further commute with each other $\left[\hat{T}_{x}^{0}, \hat{T}_{y}^{0}\right]=0$, which allows us to apply the wellknown Bloch theorem [84, 85]. In the presence of a vector potential, however, the Hamiltonian is no longer invariant under the translation by one lattice unit vector because the corresponding vector potential $\mathbf{A}_{m, n}$ is not invariant under this discrete translation even though the magnetic field $\mathbf{B}$ itself might be. Hence, the translation operators $\hat{T}_{i}^{0}$ do not commute with Hamiltonian (2.2). For the following discussion it is convenient to write Hamiltonian (2.2) in the following form

$$
\hat{H}=\hat{T}_{x}+\hat{T}_{y}+\text { h.c., }
$$

where $\hat{T}_{x}$ and $\hat{T}_{y}$ describe the translation by one lattice constant along the $x$ - and $y$ direction in the presence of a vector potential $\mathbf{A}_{m, n}$ :

$$
\hat{T}_{x}=\sum_{m, n} \hat{a}_{m+1, n}^{\dagger} \hat{a}_{m, n} \mathrm{e}^{i \phi_{m, n}^{x}}, \quad \hat{T}_{y}=\sum_{m, n} \hat{a}_{m, n+1}^{\dagger} \hat{a}_{m, n} \mathrm{e}^{i \phi_{m, n}^{y}} .
$$

For simplicity the tunnel coupling is set to $J=-1$. It can be easily verified that in general the operators $\hat{T}_{i}$ do not commute with each other $\left[\hat{T}_{x}, \hat{T}_{y}\right] \neq 0$, thus, they neither 


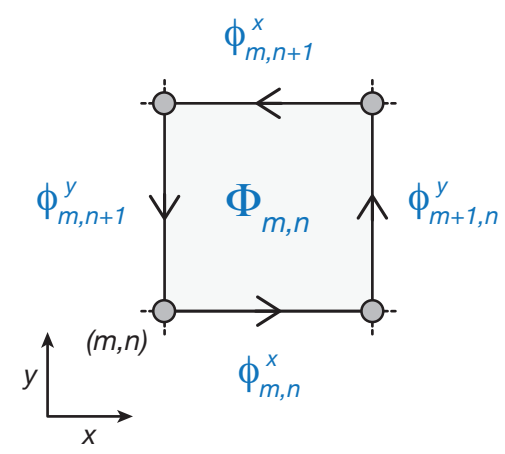

Figure 2.2: Effective magnetic flux $\Phi_{m, n}$ per plaquette. The vector potential $\mathbf{A}_{m, n}$ gives rise to and effective flux per plaquette (gray shaded area) as defined in Eq. (2.10), with the convention that the magnetic field is pointing along the $+\hat{e}_{z}$-direction. The arrows illustrate the direction of the tunneling.

commute with the Hamiltonian, $\left[\hat{T}_{x}, \hat{H}\right] \neq 0$ and $\left[\hat{T}_{y}, \hat{H}\right] \neq 0$. To find the new symmetries of the lattice Hamiltonian with flux and to recover translational invariance new operators have to be constructed, which form a complete set of commuting operators with Hamiltonian (2.6). These operators are a combination of translation and gauge transformation

$$
\hat{T}_{x}^{M}=\sum_{m, n} \hat{a}_{m+1, n}^{\dagger} \hat{a}_{m, n} \mathrm{e}^{i \theta_{m, n}^{x},} \quad \hat{T}_{y}^{M}=\sum_{m, n} \hat{a}_{m, n+1}^{\dagger} \hat{a}_{m, n} \mathrm{e}^{i \theta_{m, n}^{y}},
$$

and are called magnetic translations operators (MTOs) [87-89]. In general the new magnetic translation symmetry will differ from the one of the underlying lattice potential. The phases $\theta_{m, n}^{i}$ are determined by the formal requirement that the MTOs have to commute with the Hamiltonian, $\left[\hat{T}_{i}^{M}, \hat{H}\right]=0$, leading to

$$
\theta_{m, n}^{x}=\phi_{m, n}^{x}+\Phi_{m, n} n, \quad \theta_{m, n}^{y}=\phi_{m, n}^{y}-\Phi_{m, n} m .
$$

A detailed derivation of these expressions can be found in Appendix A, which closely follows Ref. [90]. The flux per unit cell $\Phi_{m, n}$ is allowed to vary across the lattice and its index is determined by the lattice site on the lower left corner (Fig. 2.2)

$$
\Phi_{m, n}=\phi_{m, n}^{x}+\phi_{m+1, n}^{y}-\phi_{m, n+1}^{x}-\phi_{m, n}^{y} .
$$

Although the derivation of Eq. (2.9) was carried out for general flux distributions $\Phi_{m, n}$, it might not be directly applicable in the case of more complicated configurations such as staggered flux distributions (chapter 5). In this case the corresponding MTOs can still be derived using the methods described in Appendix A. The MTOs obtained in this way do commute with the Hamiltonian by construction but they do not necessarily commute with each other. The value of the commutator $\left[\hat{T}_{x}^{M}, \hat{T}_{y}^{M}\right]$ can be computed using the single-particle state $\psi_{i, j}=\hat{a}_{i, j}^{\dagger}|0\rangle$ on lattice site $(i, j)$ : 


$$
\begin{aligned}
& \hat{T}_{x}^{M} \hat{T}_{y}^{M} \psi_{i, j}=\hat{T}_{x}^{M} \mathrm{e}^{i \theta_{i, j}^{y}} \psi_{i, j+1}=\mathrm{e}^{i\left(\theta_{i, j+1}^{x}+\theta_{i, j}^{y}\right)} \psi_{i+1, j+1} \\
& \hat{T}_{y}^{M} \hat{T}_{x}^{M} \psi_{i, j}=\hat{T}_{y}^{M} \mathrm{e}^{i \theta_{i, j}^{x}} \psi_{i+1, j}=\mathrm{e}^{i\left(\theta_{i, j}^{x}+\theta_{i+1, j}^{y}\right)} \psi_{i+1, j+1} .
\end{aligned}
$$

The specific form of the MTOs depends on the particular form of the vector potential $\mathbf{A}_{m, n}$, which in turn depends on the choice of gauge. However, there is no fundamental reason for the two magnetic translations operators $\hat{T}_{x}^{M}$ and $\hat{T}_{y}^{M}$ defined in Eq. (2.8) to commute with each other.

\subsubsection{Homogeneous magnetic fields}

Let us consider a homogeneous magnetic field with $\Phi_{m, n} \equiv \Phi=2 \pi \alpha$ per plaquette (Fig. 2.3a). Inserting Eq. (2.9) into the expressions given in Eq. (2.11) and (2.12) leads to the following result

$$
\mathrm{e}^{-i \Phi} \hat{T}_{x}^{M} \hat{T}_{y}^{M}=\hat{T}_{y}^{M} \hat{T}_{x}^{M}
$$

Consequently, the commutator vanishes only if $\Phi$ is an integer multiple of $2 \pi$. Such a flux configuration is however gauge-equivalent to the trivial case of zero flux per plaquette and does not correspond to the situation we are interested in. Nevertheless equation (2.13) does provide us with an intuitive picture of the MTOs by acting with them on a single-particle state $\psi_{i, j}$ around the borders of one lattice unit cell that is pierced by a flux $\Phi$. Choosing the direction illustrated in Fig. 2.3b the single particle state $\psi_{i, j}$ picks up a phase $-\Phi$, which corresponds to a flux pointing in the opposite direction.

For flux values different from $\Phi=v \times 2 \pi, v \in \mathbb{Z}$, this intuitive picture suggests that commuting magnetic translation operators can be constructed if they enclose a supercell on the lattice pierced by a magnetic flux equal to an integer multiple of $2 \pi$. For a super-cell of dimension $k \times l$ one obtains

$$
\begin{aligned}
\left(\hat{T}_{x}^{M}\right)^{k}\left(\hat{T}_{y}^{M}\right)^{l} \psi_{i, j} & =\left(\hat{T}_{x}^{M}\right)^{k} \exp \left(i \sum_{v=0}^{l-1} \theta_{i, j+v}^{y}\right) \psi_{i, j+l} \\
& =\exp \left(i \sum_{\mu=0}^{k-1} \theta_{i+\mu, j+l}^{x}+i \sum_{v=0}^{l-1} \theta_{i, j+v}^{y}\right) \psi_{i+k, j+l}, \\
\left(\hat{T}_{y}^{M}\right)^{l}\left(\hat{T}_{x}^{M}\right)^{k} \psi_{i, j} & =\left(\hat{T}_{y}^{M}\right)^{l} \exp \left(i \sum_{\mu=0}^{k-1} \theta_{i+\mu, j}^{x}\right) \psi_{i+k, j} \\
& =\exp \left(i \sum_{v=0}^{l-1} \theta_{i+k, j+v}^{y}+i \sum_{\mu=0}^{k-1} \theta_{i+\mu, j}^{x}\right) \psi_{i+k, j+l} .
\end{aligned}
$$



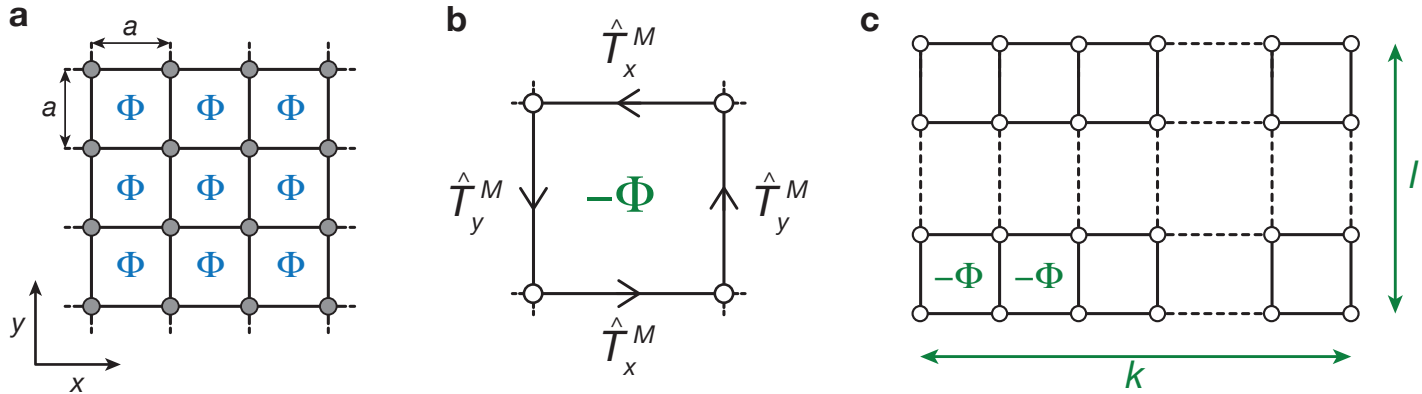

Figure 2.3: Magnetic translation operators for a homogeneous magnetic field. a Schematic drawing of a square lattice with lattice constant $a$ and homogeneous flux $\Phi$ per plaquette. $\mathbf{b}$ Action of the magnetic translation operators along a closed path around one lattice unit cell, $\left(\hat{T}_{y}^{M}\right)^{\dagger}\left(\hat{T}_{x}^{M}\right)^{\dagger} \hat{T}_{y}^{M} \hat{T}_{x}^{M} \psi_{i, j}=\exp (-i \Phi) \psi_{i j}$. c If the path shown in (b) is enlarged around a supercell of dimension $k \times l$ the action of the MTOs along that path corresponds to a phase shift of $-k l \Phi=-2 \pi \alpha \cdot k l$.

Hence, the phase acquired by the single-particle state $\psi_{i, j}$ which was translated along the borders of the super-cell by acting on it with the MTOs is simply given by the sum of the corresponding phase terms $\theta_{m, n}^{i}$ along the borders of the super-cell. This sum can be decomposed in $k \cdot l$ lattice unit cells, for which the phase term was determined in Eq. (2.13), and one obtains

$$
\mathrm{e}^{-i k l \Phi}\left(\hat{T}_{x}^{M}\right)^{k}\left(\hat{T}_{y}^{M}\right)^{l}=\left(\hat{T}_{y}^{M}\right)^{l}\left(\hat{T}_{x}^{M}\right)^{k}
$$

as illustrated in Fig. 2.3c. For rational values of $\alpha=p / q(p, q \in \mathbb{Z})$ the commutator vanishes if

$$
k l \Phi=2 \pi p \frac{k l}{q} \stackrel{!}{=} 2 \pi \times v, \quad v \in \mathbb{Z} .
$$

The smallest possible super-cell for which $\left[\left(\hat{T}_{x}^{M}\right)^{k},\left(\hat{T}_{y}^{M}\right)^{l}\right]=0$ is given by $k l=q$ and is called magnetic unit cell. The area of the magnetic unit cell $A_{M U}$ is $q$ times larger than the area of the normal lattice unit cell and contains $q$ sites. The new operators $\left(\hat{T}_{x}^{M}\right)^{k} \equiv \hat{M}_{x}^{k}$ and $\left(\hat{T}_{y}^{M}\right)^{l} \equiv \hat{M}_{y}^{l}$ together with $\hat{H}$ (2.6) form a complete set of commuting operators such that one can find simultaneous eigenstates $\Psi_{m, n}$ by formulating a generalized Bloch theorem based on the magnetic translation symmetries:

$$
\begin{aligned}
& \hat{M}_{x}^{k} \Psi_{m, n}=\mathrm{e}^{i \mu_{m, n}^{x}} \Psi_{m+k, n}=\mathrm{e}^{i k_{x} k a} \Psi_{m, n}, \\
& \hat{M}_{y}^{l} \Psi_{m, n}=\mathrm{e}^{i \mu_{m, n}^{y}} \Psi_{m, n+l}=\mathrm{e}^{i k_{y} l a} \Psi_{m, n},
\end{aligned}
$$

with $k l=q, a$ the lattice constant and $\mathbf{k}=\left(k_{x}, k_{y}\right)$ defined within the first magnetic Brillouin zone (FBZ): $-\pi /(k a) \leq k_{x}<\pi /(k a),-\pi /(l a) \leq k_{y}<\pi /(l a)$. An explicit form of the eigenstates will be derived in the following section for $\alpha=1 / 4$. Note that the 

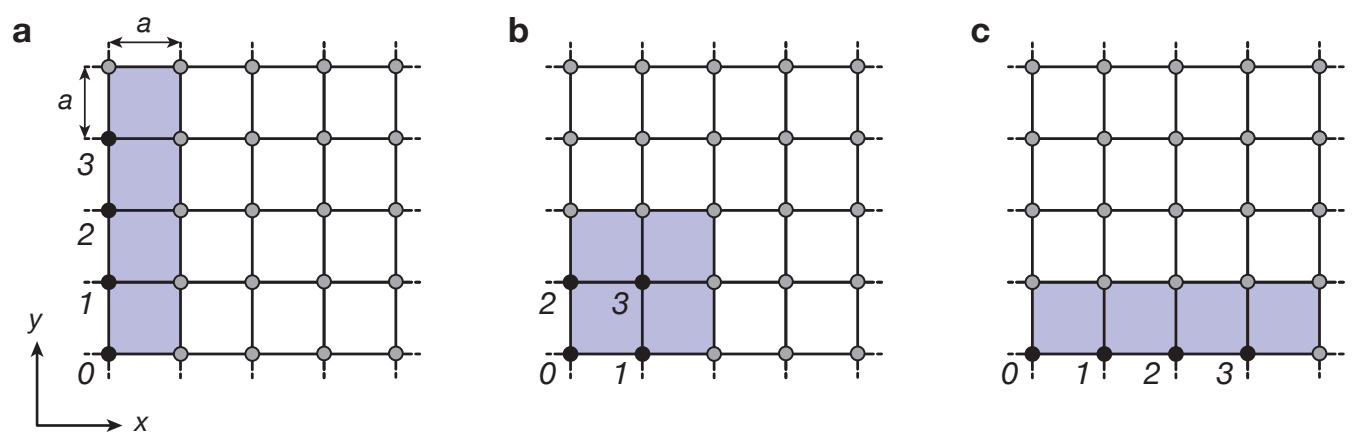

Figure 2.4: Magnetic unit cells of a square lattice with flux $\alpha=1 / 4$. Schematic drawing of a square lattice with constant $a$. The area of the magnetic unit cell (blue shaded are) depends on the magnetic flux; for $\alpha=1 / 4$ it contains $q=4$ sites (black circles) and covers an area of four lattice unit cells $A_{M U}=4 a^{2}$. There are three different possibilities to choose its shape: a rectangular and oriented along $y$ with $A_{M U}=1 a \times 4 a, \mathbf{b}$ symmetric with $A_{M U}=2 a \times 2 a$ and $\mathbf{c}$ rectangular and oriented along $x$ with $A_{M U}=4 a \times 1 a$.

area of the magnetic unit cell is fixed by the strength of the magnetic flux $\alpha=p / q$, its dimensions, however, are not.

\subsubsection{Magnetic translation operators for $\alpha=1 / 4$}

In this section the MTOs for $\alpha=1 / 4$ are introduced. According to Eq. (2.17) the magnetic unit cell consists of four lattice unit cells, such that its area is given by $A_{M U}=4 a^{2}$. For this value of the flux there are three different possibilities to choose its dimensions (Fig. 2.4). The specific form of the MTOs is gauge dependent, therefore the following example is carried out choosing the Landau gauge $\phi_{m, n}=(-2 \pi \alpha n, 0)$. The noncommuting magnetic translation operators in this gauge are

$$
\hat{T}_{x}^{M}=\sum_{m, n} \hat{a}_{m+1, n}^{\dagger} \hat{a}_{m, n}, \quad \hat{T}_{y}^{M}=\sum_{m, n} \hat{a}_{m, n+1}^{\dagger} \hat{a}_{m, n} \mathrm{e}^{-i 2 \pi \alpha m},
$$

and the commuting ones are given by

$$
\hat{M}_{x}^{k}=\sum_{m, n} \hat{a}_{m+k, n}^{\dagger} \hat{a}_{m, n}, \quad \hat{M}_{y}^{l}=\sum_{m, n} \hat{a}_{m, n+l}^{\dagger} \hat{a}_{m, n} \mathrm{e}^{-i 2 \pi \alpha m l},
$$

with $k l=4$. In the following explicit forms of the eigenfunctions for the different choices of the magnetic unit cell are determined.

Rectangular magnetic unit cell oriented along y (Fig. 2.4a): In the literature this is the most common choice for the magnetic unit cell in the Landau gauge because the MTOs take the form of usual lattice translation operators

$$
\hat{M}_{x}^{1}=\sum_{m, n} \hat{a}_{m+1, n}^{\dagger} \hat{a}_{m, n}, \quad \hat{M}_{y}^{4}=\sum_{m, n} \hat{a}_{m, n+4}^{\dagger} \hat{a}_{m, n},
$$


and the eigenfunctions satisfying the generalized Bloch's theorem in Eq. (2.18) can be written in the following form

$$
\Psi_{m, n}=\mathrm{e}^{i k_{x} m a} \mathrm{e}^{i k_{y} n a} \psi_{n}, \quad \psi_{n+4}=\psi_{n},
$$

where $\Psi_{m, n}$ is expanded in single-particle on-site wave functions; $\psi_{i}, i=\{0,1,2,3\}$, is the complex amplitude of the wave function on the four sites of the magnetic unit cell and $\mathbf{k}$ is defined within the FBZ: $-\pi / a \leq k_{x}<\pi / a,-\pi /(4 a) \leq k_{y}<\pi /(4 a)$. One can verify that this ansatz fulfills the generalized form of Bloch's theorem (2.18)

$$
\begin{aligned}
& \hat{M}_{x}^{1} \Psi_{m, n}=\Psi_{m+1, n}=\mathrm{e}^{i k_{x} a} \Psi_{m, n}, \\
& \hat{M}_{y}^{4} \Psi_{m, n}=\Psi_{m, n+4}=\mathrm{e}^{4 i k_{y} a} \Psi_{m, n} .
\end{aligned}
$$

Symmetric magnetic unit cell (Fig. 2.4b): For the square symmetric magnetic unit cell the MTO along $y$ is slightly more complicated. It is a combination of a usual translation by two lattice sites and an additional phase factor,

$$
\hat{M}_{x}^{2}=\sum_{m, n} \hat{a}_{m+2, n}^{\dagger} \hat{a}_{m, n}, \quad \hat{M}_{y}^{2}=\sum_{m, n} \hat{a}_{m, n+2}^{\dagger} \hat{a}_{m, n} \mathrm{e}^{-i \pi m} .
$$

In this case the wave function has to fulfill the following relations

$$
\begin{aligned}
& \hat{M}_{x}^{2} \Psi_{m, n}=\Psi_{m+2, n}=\mathrm{e}^{2 i k_{x} a} \Psi_{m, n} \\
& \hat{M}_{y}^{2} \Psi_{m, n}=\mathrm{e}^{-i \pi m} \Psi_{m, n+2}=\mathrm{e}^{2 i k_{y} a} \Psi_{m, n} .
\end{aligned}
$$

For $m$ even the eigenfunctions take the usual form Bloch functions; for $m$ odd, however, additional phase terms have to be introduced. The combined solution can be written as

$$
\Psi_{m, n}=\mathrm{e}^{i k_{x} m a} \mathrm{e}^{i k_{y} n a} \begin{cases}\psi_{0}, & \text { for } m, n \text { even } \\ \psi_{1} \mathrm{e}^{-i n \pi / 2}, & \text { for } m \text { odd, } n \text { even } \\ \psi_{2}, & \text { for } m \text { even, } n \text { odd } \\ \psi_{3} \mathrm{e}^{-i n \pi / 2}, & \text { for } m, n \text { odd }\end{cases}
$$

with $\mathbf{k}$ defined within the FBZ, $-\pi /(2 a) \leq k_{x}<\pi /(2 a)$ and $-\pi /(2 a) \leq k_{y}<\pi /(2 a)$.

Rectangular magnetic unit cell oriented along x (Fig. 2.4c): The third possible choice is a rectangular magnetic unit cell oriented along $x$, where the corresponding MTOs are

$$
\hat{M}_{x}^{4}=\sum_{m, n} \hat{a}_{m+4, n}^{\dagger} \hat{a}_{m, n}, \quad \hat{M}_{y}^{1}=\sum_{m, n} \hat{a}_{m, n+1}^{\dagger} \hat{a}_{m, n} \mathrm{e}^{-i \pi m / 2} .
$$


For this choice of the magnetic unit cell, the translation along $x$ is again of the usual form but the one along $y$ is not. For every site $m$ an additional phase term has to be introduced in $\Psi_{m, n}$ to fulfill Bloch's theorem (2.18)

$$
\begin{aligned}
& \hat{M}_{x}^{4} \Psi_{m, n}=\Psi_{m+4, n}=\mathrm{e}^{4 i k_{x} a} \Psi_{m, n}, \\
& \hat{M}_{y}^{1} \Psi_{m, n}=\mathrm{e}^{-i \pi m / 2} \Psi_{m, n+1}=\mathrm{e}^{i k_{y} a} \Psi_{m, n} .
\end{aligned}
$$

The form of the eigenfunctions involves an additional phase factor that depends on the site index $(m, n)$ and can be written as

$$
\Psi_{m, n}=\mathrm{e}^{i k_{x} m a} \mathrm{e}^{i k_{y} n a} \mathrm{e}^{i \pi m n / 2} \psi_{m}, \quad \psi_{m+4}=\psi_{m},
$$

where $\mathbf{k}$ is defined in the range $-\pi /(4 a) \leq k_{x}<\pi /(4 a)$ and $-\pi / a \leq k_{y}<\pi / a$.

\subsection{Harper-Hofstadter Hamiltonian}

The theoretical description of a lattice Hamiltonian with flux depends on the choice of the gauge since the explicit form of the MTOs depends on the particular form of the vector potential as was shown above. The physical observables investigated in the following (energy spectrum, topological invariants) are gauge independent and one can choose a vector potential where the calculations are particularly simple. As in the previous section the vector potential will be written in the Landau gauge $\phi_{m, n}=(-\Phi n, 0)$, which corresponds to a uniform magnetic field with flux $\Phi=2 \pi \alpha$ per plaquette

$$
\hat{H}=-J \sum_{m, n}\left(\mathrm{e}^{-i \Phi n} \hat{a}_{m+1, n}^{\dagger} \hat{a}_{m, n}+\hat{a}_{m, n+1}^{\dagger} \hat{a}_{m, n}+\text { h.c. }\right) \text {. }
$$

In this gauge only tunneling along the $x$-direction is complex while tunneling along the $y$-direction is real. This Hamiltonian is known as the famous Harper-Hofstadter Hamiltonian [74-76], whose single-particle energy spectrum exhibits a fractal self-similar structure as a function of the flux $\alpha$, known as Hofstadter's butterfly [76].

\subsubsection{Single particle energy spectrum}

In order to solve the Schrödinger equation first the commuting magnetic translation operators need to be constructed using the MTOs given in Eq. (2.19), which were derived in the same gauge. For rational values of the flux $\alpha=p / q$ one can always choose a magnetic unit cell oriented along the $y$-direction with dimensions $(1 \times q) \cdot a^{2}$ (see also Fig. 2.4a for $\alpha=1 / 4)$. For this particular choice of the magnetic unit cell the commuting MTOs can be written in the following form,

$$
\hat{M}_{x}^{1}=\sum_{m, n} \hat{a}_{m+1, n}^{+} \hat{a}_{m, n}, \quad \hat{M}_{y}^{q}=\sum_{m, n} \hat{a}_{m, n+q}^{+} \hat{a}_{m, n} .
$$


Both operators are equivalent to the usual lattice translation operators as defined in Eq. (2.5), where the one along $x$ corresponds to a translation by one lattice constant and the one along $y$ by $q$ lattice constants. The magnetic unit cell contains a flux $\Phi_{M U}=$ $p \times 2 \pi$. In order to solve the Schrödinger equation one can make the following ansatz for the wave function

$$
\Psi_{m, n}=\mathrm{e}^{i k_{x} m a} \mathrm{e}^{i k_{y} n a} \psi_{n}, \quad \psi_{n+q}=\psi_{n},
$$

where $k_{x}, k_{y}$ are defined in the range $-\pi / a \leq k_{x}<\pi / a$ and $-\pi /(q a) \leq k_{y}<\pi /(q a)$. As shown above, this ansatz fulfills the generalized Bloch theorem (2.18)

$$
\begin{aligned}
& \hat{M}_{x}^{1} \Psi_{m, n}=\Psi_{m+1, n}=\mathrm{e}^{i k_{x} a} \Psi_{m, n}, \\
& \hat{M}_{y}^{q} \Psi_{m, n}=\Psi_{m, n+q}=\mathrm{e}^{i k_{y} g a} \Psi_{m, n} .
\end{aligned}
$$

By inserting Eq. (2.32) into the Schrödinger equation associated with the Harper-Hofstadter Hamiltonian (2.30)

$$
E \Psi_{m, n}=-J\left(\mathrm{e}^{-i \Phi n} \Psi_{m+1, n}+\mathrm{e}^{i \Phi n} \Psi_{m-1, n}+\Psi_{m, n+1}+\Psi_{m, n-1}\right)
$$

one obtains the following simplified equation

$$
E \psi_{n}=-J\left[2 \cos \left(k_{x} a-\Phi n\right) \psi_{n}+\mathrm{e}^{i k_{y} a} \psi_{n+1}+\mathrm{e}^{-i k_{y} a} \psi_{n-1}\right] .
$$

Consequently, the problem reduces to a $q$-dimensional eigenvalue equation

$$
E(\mathbf{k})\left(\begin{array}{c}
\psi_{0} \\
\psi_{1} \\
\vdots \\
\psi_{q-1}
\end{array}\right)=H(\mathbf{k})\left(\begin{array}{c}
\psi_{0} \\
\psi_{1} \\
\vdots \\
\psi_{q-1}
\end{array}\right)
$$

where the $q \times q$ matrix is defined as

$$
H(\mathbf{k})=-J\left(\begin{array}{ccccc}
h_{0} & \mathrm{e}^{i k_{y} a} & 0 & \cdots & \mathrm{e}^{-i k_{y} a} \\
\mathrm{e}^{-i k_{y} a} & h_{1} & \mathrm{e}^{i k_{y} a} & \cdots & 0 \\
0 & \mathrm{e}^{-i k_{y} a} & h_{2} & \cdots & 0 \\
\vdots & \vdots & \vdots & \ddots & \vdots \\
\mathrm{e}^{i k_{y} a} & 0 & 0 & \cdots & h_{q-1}
\end{array}\right),
$$

with $h_{q}=2 \cos \left(k_{x} a-q \Phi\right)$. Without magnetic field, or more generally for $\alpha \in \mathbb{Z}$, one obtains a single energy band with dispersion relation

$$
E(\mathbf{k})=-2 J \cos \left(k_{x} a\right)-2 J \cos \left(k_{y} a\right),
$$




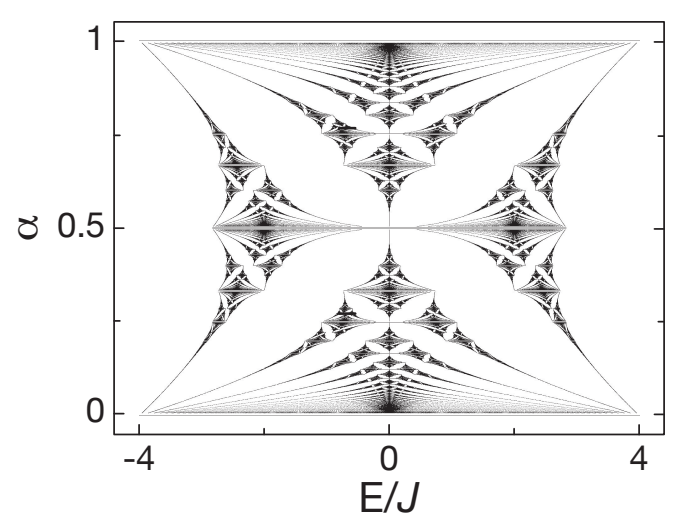

Figure 2.5: Single-particle energy spectrum of an electron in a periodic potential exposed to large magnetic fields, known as Hofstadter's butterfly. Energy spectrum of the lowest tightbinding band as a function of the flux per unit cell $\alpha=\Phi /(2 \pi)$ displaying a fractal, self-similar structure. The number of energy bands depends crucially on the value of the flux per lattice unit cell. (Data taken from Ref. [76])

where the corresponding bandwidth is given by $E_{\mathrm{bw}}=2 \times 4 \mathrm{~J}$. In the presence of a rational flux per plaquette $\alpha=p / q$ this band splits into $q$ subbands (Fig. 2.5) with dispersion relations $E_{\mu}(\mathbf{k}), \mu=\{1, \ldots, q\}$. This leads to the famous fractal structure of the Hofstadter butterfly which displays the single-particle energy as a function of the magnetic flux $\alpha$. In Figure 2.6 two examples of such a spectrum are shown for $\alpha=1 / 5$ and $\alpha=1 / 6$, which were computed using Eqs. (2.36)-(2.37).

For irrational values of the flux the spectrum splits into an infinite number of energy levels forming a Cantor set [76]. Similar spectra were further computed for graphenetype lattices [91, 92]. The nature of the single-particle energy spectrum is determined by rational and irrational values of $\alpha$ respectively. In a Penrose lattice two kinds of elementary tilings may exist such that one of them is pierced by a rational flux $\alpha$ while the second one is pierced by an irrational one. It has been shown that such a configuration can lead to interesting electronic properties and the spectrum is butterfly-like with a periodicity that is characteristic of the underlying quasicrystal [93].

\subsubsection{Particle-hole symmetry}

An important property of the Hofstadter model is the particle-hole symmetry, which gives rise to certain symmetries in the energy spectrum and the Chern number distribution of the energy bands (Sect. 2.4). Let us consider the following transformation

$$
\Psi_{m, n} \quad \rightarrow \quad \tilde{\Psi}_{m, n}=(-1)^{m+n} \Psi_{m, n} .
$$

The new wave function $\tilde{\Psi}_{m, n}$ satisfies the Harper equation (2.34) 

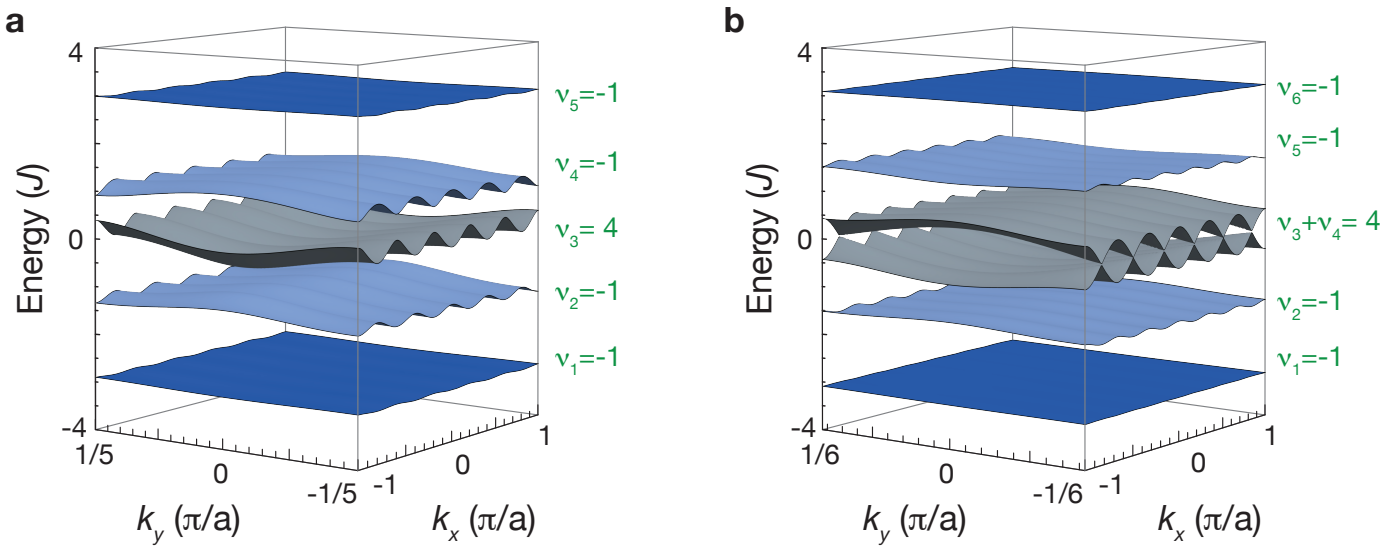

Figure 2.6: Single-particle energy spectrum and Chern number distribution of the Hofstadter model for $\alpha=1 / 5$ (a) and $\alpha=1 / 6$ (b). For $\alpha=1 / 5$ the spectrum splits into five subbands, while for $\alpha=1 / 6$ it splits into six. The Hamiltonian is particle-hole symmetric, which manifests itself in certain symmetries between the dispersion relations of the individual subbands (Sect. 2.3.2). It further leads to a symmetric Chern number distribution around $E=0$.

$$
-E \tilde{\Psi}_{m, n}=-J\left(\mathrm{e}^{-i \Phi n} \tilde{\Psi}_{m+1, n}+\mathrm{e}^{i \Phi n} \tilde{\Psi}_{m-1, n}+\tilde{\Psi}_{m, n+1}+\tilde{\Psi}_{m, n-1}\right),
$$

which is similar to Eq. (2.34) but with different energy $E \rightarrow-E$. This means that if there exists a state $\Psi_{m, n}$ with energy $E$ there necessarily also exists a state $\tilde{\Psi}_{m, n}$ with opposite energy $-E$. This result illustrates the particle-hole symmetry present in the system. The state can be also written in the following way

$$
\tilde{\Psi}_{m, n}=\mathrm{e}^{i k_{x} m a} \mathrm{e}^{i k_{y} n a} \tilde{\psi}_{n}, \quad \tilde{\psi}_{n+q}=\tilde{\psi}_{n} .
$$

Inserting this state into Eq. (2.40) leads to an eigenvalue equation for the new periodic function $\tilde{\psi}_{n}$, which can be written as follows

$$
E \tilde{\psi}_{n}=-J\left[2 \cos \left(k_{x} a+\pi-\Phi n\right) \tilde{\psi}_{n}+\mathrm{e}^{i\left(k_{y} a+\pi\right)} \tilde{\psi}_{n+1}+\mathrm{e}^{-i\left(k_{y} a+\pi\right)} \tilde{\psi}_{n-1}\right] .
$$

Comparing this result with Eq. (2.35) shows that the eigenstate associated with the band $E(\mathbf{k})$ located at positive energies is related to the state in the lower band at negative energies through the relation

$$
\psi_{n}\left(k_{x}, k_{y}\right)=\tilde{\psi}_{n}\left(k_{x}+\pi / a, k_{y}+\pi / a\right) .
$$

Consequently the particle-hole transformation (2.39) maps a state at energy $+E$ to a state at energy $-E$. Additionally this transformation corresponds to a shift in momentum space $\left(k_{x}, k_{y}\right) \rightarrow\left(k_{x}+\pi / a, k_{y}+\pi / a\right)$. Hence, the dispersion relation of a band $\mu$ which 
is located around a mean energy $\bar{E}_{\mu}>0$ is related to the dispersion relation of a band $\tilde{\mu}$ located around a mean value $\bar{E}_{\tilde{\mu}}<0$ according to

$$
E_{\mu}\left(k_{x}, k_{y}\right)=-E_{\tilde{\mu}}\left(k_{x}+\pi / a, k_{y}+\pi / a\right) .
$$

In addition the particle-hole symmetry has important consequences for the Chern number distribution as will be discussed in the following section.

\subsection{Chern number}

The topology of an energy band is robust against continuous deformations of the underlying Hamiltonian and is characterized by topological invariants. These topological properties can have important physical consequences. It has been shown that the quantization of the Hall conductance discovered by Klaus von Klitzing et al. in 1980 [2] is directly related to an integer topological invariant known as the Chern number [8].

In solid-state experiments the quantization of the Hall conductance $\sigma_{H}$ is observed by sending a constant current through the sample and measuring the voltage difference in the transverse direction. At low temperatures all energy bands below the Fermi energy $E_{F}$ are filled. If the Fermi energy lies within a spectral gap the Hall conductance is determined by

$$
\sigma_{H}=\frac{e^{2}}{h} \sum_{E_{\mu}<E_{F}} v_{\mu}
$$

where $v_{\mu}$ is the Chern number of the $\mu$-th band $E_{\mu}$ and the sum runs over all occupied bands below the Fermi energy $E_{\mu}<E_{F}$.

A QH device is insulating in the bulk if the Fermi energy is located in an energy gap and the value of the Hall conductivity is determined by the number of gapless chiral edge states, that are contributing to the current. The existence of these modes can be seen as a manifestation of the topological order of the bulk. The connection between the topological properties of the bulk energy bands and the quantization of the Hall conductance was first identified in the work by Thouless, Kohmoto, Nightingale, den Nijs [8]. The corresponding topological invariant, the Chern number of the $\mu$-th energy band can be expressed in terms of the periodic eigenfunctions $\left|u_{\mu}(\mathbf{k})\right\rangle$, which are solutions of the eigenvalue equation (2.36) given above,

$$
v_{\mu}=\frac{i}{2 \pi} \int_{F B Z} \underbrace{\left(\left\langle\frac{\partial u_{\mu}(\mathbf{k})}{\partial k_{x}} \mid \frac{\partial u_{\mu}(\mathbf{k})}{\partial k_{y}}\right\rangle-\left\langle\frac{\partial u_{\mu}(\mathbf{k})}{\partial k_{y}} \mid \frac{\partial u_{\mu}(\mathbf{k})}{\partial k_{x}}\right\rangle\right)}_{=-i \Omega_{\mu}(\mathbf{k})} \mathrm{d}^{2} k,
$$

where $\Omega_{\mu}(\mathbf{k})$ is know as the Berry curvature of the $\mu$-th band [94] and the integral is carried out over the first magnetic Brillouin zone. This invariant was derived for an infinite system without edges. Using this expression the topological role of the edge states 
is not yet clear. The relation between the topological properties of the edge modes and the bulk is commonly denoted as bulk-edge correspondence [10-12]. In particular, it was shown by Hatsugai, that the topology of the edge states is characterized by an integer, which is equal to the bulk topological invariant [10,11].

In chapter 8 we are going to present an experimental observation of the Chern number of the lowest Hofstadter band for $\alpha=1 / 4$ with bosonic atoms [73]. In these measurements the topological properties of the bulk were probed through measurements of the anomalous Hall velocity, which occurs transverse to an applied force and is proportional to the Berry curvature defined above [94].

\subsubsection{Distribution in the Hofstadter model}

The particle-hole symmetry (Sect. 2.3.2) inherent to the Hofstadter model has important consequences on the Chern number distribution $v_{\mu}$ of the Hofstadter bands. Taking into account the symmetry properties of the wave-function amplitudes $\psi_{n}$ in equation (2.43) leads to an equivalent relation for the eigenfunctions

$$
\left|u_{\mu}\left(k_{x}, k_{y}\right)\right\rangle=\left|u_{\tilde{\mu}}\left(k_{x}+\pi / a, k_{y}+\pi / a\right)\right\rangle .
$$

As a consequence the Berry curvature of the $\mu$-the band is related the Berry curvature of the opposite band $\tilde{\mu}$ according to

$$
\Omega_{\mu}\left(k_{x}, k_{y}\right)=\Omega_{\tilde{\mu}}\left(k_{x}+\pi / a, k_{y}+\pi / a\right) .
$$

Hence, both bands share the same Berry curvature shifted in momentum space by $\left(\delta k_{x}, \delta k_{y}\right)=(\pi / a, \pi / a)$. The characteristic Chern number $v_{\mu}$ of the band defined in Eq. (2.46) is obtained by integrating the Berry curvature over the first magnetic Brillouin zone. As a result the two bands share the same Chern number $v_{\mu}=v_{\tilde{\mu}}$ and the distribution is symmetric around $E=0$ (Fig. 2.6). Note, that the Chern number of the total tight-binding band necessarily vanishes, i.e.

$$
\sum_{\mu} v_{\mu}=0
$$

\subsubsection{Diophantine equation}

It has been shown that the fractal structure of the Hofstadter butterfly follows a simply relation, which allows for an analytical computation of the Chern number. For a rational flux $\alpha=p / q$ the energy gaps are characterized by two integers $s_{r}$ and $t_{r}$, which are determined by a Diophantine equation $[95,96]$

$$
r=q s_{r}+p t_{r}, \quad\left|t_{r}\right| \leq \frac{q}{2}, \quad s_{r}, t_{r} \in \mathbb{Z},
$$


where $r$ denotes the $r$-th energy gap of the Hofstadter spectrum. Since the spectrum is split into $q$-subbands, $r$ can only take values in the interval $0 \leq r \leq q$. The solutions of equation Eq. (2.50) are uniquely defined and the two numbers $s_{r}, t_{r}$ are topological numbers characterizing the gap, where the integer $t_{r}$ determines the value of the Hall conductivity $[8,97,98]$ according to

$$
\sigma_{H}=-\frac{e^{2}}{h} t_{r}
$$

Thus, $t_{r}$ is given by the sum of the Chern numbers $\sum_{1}^{r} v_{r}$ of all occupied bands and the $r$ th band, which lies between the $r$ th and the $(r-1)$ st energy gap, carries an integral Hall conductance determined by the Chern number of the $r$-th band $v_{r}$ which is a solution of the following Diophantine equation

$$
-1=q\left(s_{r-1}-s_{r}\right)+p\left(t_{r-1}-t_{r}\right)=q \sigma_{r}+p v_{r}, \quad \sigma_{r} \in \mathbb{Z} .
$$

For generic values of the flux with $p=1$ one can show that the bands with $r<q / 2$ exhibit a Chern number $v_{r}=-1$.

For $r$ even, the middle two bands touch at $q$ Dirac cones (Fig. 2.6b) and the Diophantine equation above can only predict the sum of the two Chern numbers $v_{r}=q-2$. For $r$ odd, the middle band carries a Chern number $v_{r}=q-1$. These analytical results are in agreement with the numerical calculations depicted in Fig. 2.6.

A Chern number of the lowest band larger than one $\left|v_{1}\right|>1$ can be achieved e.g. with a flux $\alpha=4 / 9$, where the Chern number of the lowest band is $v_{1}=2$.

\subsubsection{Numerical calculation of the Chern number}

An efficient way to calculate the Berry curvature and the Chern number of non-degenerate bands was proposed by Fukui et al. [99] and will be briefly reviewed here. For the sake of simplicity the band index $\mu$ is omitted in the following discussion. The fundamental idea is to compute the Berry curvature numerically by discretizing the Brillouin zone using a grid in momentum space defined according to

$$
\begin{aligned}
\mathbf{k}_{\alpha}=\left(k_{x}, k_{y}\right), \quad k_{x} & =\frac{2 \pi \alpha_{1}}{N_{1}}, \quad\left(\alpha_{1}=0, \ldots, N_{1}-1\right), \\
k_{y} & =\frac{2 \pi \alpha_{2}}{q N_{2}}, \quad\left(\alpha_{2}=0, \ldots, N_{2}-1\right),
\end{aligned}
$$

where $\alpha=\left(1, \ldots, N_{1} N_{2}\right)$ and $N_{1}, N_{2}$ define the size of the unit cell of the grid. The unit vectors of the grid in momentum space along the two directions are

$$
\hat{\mathbf{e}}_{1}=\frac{2 \pi}{N_{1}}(1,0), \quad \hat{\mathbf{e}}_{2}=\frac{2 \pi}{q N_{2}}(0,1)
$$


Using this notation the discrete distribution of the Berry curvature $\tilde{\Omega}_{12}\left(\mathbf{k}_{\alpha}\right)$ in the FBZ is determined by

$$
\tilde{\Omega}_{12}\left(\mathbf{k}_{\alpha}\right) \equiv \ln \mathcal{U}_{1}\left(\mathbf{k}_{\alpha}\right) \mathcal{U}_{2}\left(\mathbf{k}_{\alpha}+\hat{\mathbf{e}}_{1}\right) \mathcal{U}_{1}\left(\mathbf{k}_{\alpha}+\hat{\mathbf{e}}_{2}\right)^{-1} \mathcal{U}_{2}\left(\mathbf{k}_{\alpha}\right)^{-1},
$$

where $\mathcal{U}_{1,2}$ is defined as the link variable

$$
\mathcal{U}_{1}\left(\mathbf{k}_{\alpha}\right)=\frac{\left\langle u\left(\mathbf{k}_{\alpha}\right) \mid u\left(\mathbf{k}_{\alpha}+\hat{\mathbf{e}}_{1}\right)\right\rangle}{\left|\left\langle u\left(\mathbf{k}_{\alpha}\right) \mid u\left(\mathbf{k}_{\alpha}+\hat{\mathbf{e}}_{1}\right)\right\rangle\right|}, \quad \mathcal{U}_{2}\left(\mathbf{k}_{\alpha}\right)=\frac{\left\langle u\left(\mathbf{k}_{\alpha}\right) \mid u\left(\mathbf{k}_{\alpha}+\hat{\mathbf{e}}_{2}\right)\right\rangle}{\left|\left\langle u\left(\mathbf{k}_{\alpha}\right) \mid u\left(\mathbf{k}_{\alpha}+\hat{\mathbf{e}}_{2}\right)\right\rangle\right|} .
$$

From this distribution the Chern number can be simply computed by taking the sum over all possible momenta $\mathbf{k}_{\alpha}$

$$
\tilde{v}=\frac{1}{2 \pi i} \sum_{\alpha} \tilde{\Omega}_{12}\left(\mathbf{k}_{\alpha}\right) .
$$

The numerical determination of the Chern number using the above equations is accurate already for very coarse grids as demonstrated in Ref. [99]. The Chern numbers of the different bands illustrated in Fig. 2.6 were evaluated using this method.

\section{The case of band touching points}

For bands that are not well separated as it is the case for the two middle bands in Fig. 2.6b the method described above cannot be applied directly but it can be generalized as shown in Ref. [99]. Assuming that there are $M$ touching bands $E_{1}(\mathbf{k}), E_{2}(\mathbf{k}), \ldots, E_{M}(\mathbf{k})$, the link variables can be substituted by determinants of $M \times M$ matrices associated with the multiplet $\psi=\left(\left|u_{1}\right\rangle,\left|u_{2}\right\rangle, \ldots,\left|u_{M}\right\rangle\right)$

$$
\tilde{\mathcal{U}}_{\gamma}\left(\mathbf{k}_{\alpha}\right)=\frac{\operatorname{det} U_{\gamma}\left(\mathbf{k}_{\alpha}\right)}{\left|\operatorname{det} U_{\gamma}\left(\mathbf{k}_{\alpha}\right)\right|}, \quad \gamma=\{1,2\} .
$$

The $M$-dimensional matrices $U_{\gamma}\left(\mathbf{k}_{\alpha}\right)$ are defined as

$$
U_{\gamma}\left(\mathbf{k}_{\alpha}\right)=\left(\begin{array}{ccc}
\left\langle u_{1}\left(\mathbf{k}_{\alpha}\right) \mid u_{1}\left(\mathbf{k}_{\alpha}+\hat{\mathbf{e}}_{\gamma}\right)\right\rangle & \cdots & \left\langle u_{1}\left(\mathbf{k}_{\alpha}\right) \mid u_{M}\left(\mathbf{k}_{\alpha}+\hat{\mathbf{e}}_{\gamma}\right)\right\rangle \\
\vdots & \ddots & \vdots \\
\left\langle u_{M}\left(\mathbf{k}_{\alpha}\right) \mid u_{1}\left(\mathbf{k}_{\alpha}+\hat{\mathbf{e}}_{\gamma}\right)\right\rangle & \cdots & \left\langle u_{M}\left(\mathbf{k}_{\alpha}\right) \mid u_{M}\left(\mathbf{k}_{\alpha}+\hat{\mathbf{e}}_{\gamma}\right)\right\rangle
\end{array}\right) .
$$

The corresponding field strength and Chern number of the multiband is defined according to Eqs. (2.55) and (2.57). The Chern number of the middle band in Fig. 2.6b was evaluated for $\alpha=1 / 6$ using this technique. The result is in agreement with the analytical solution obtained using the Diophantine equation given in the previous section: $v_{3}+v_{4}=q-2=4$. 


\section{Chapter 3}

\section{Artificial gauge fields with laser-assisted tunneling}

In ultracold-atom setups the physics of magnetic field effects cannot be simulated directly because of the charge neutrality of the atoms. Therefore new experimental methods had to be developed to circumvent this limitation by designing effective systems whose dynamics are governed by a Hamiltonian analog to the one of a charged particle in a magnetic field $[58,59]$. One possibility is to exploit the equivalence between the Lorentz force and the Coriolis force in rotating systems [56, 57], which was successfully implemented in several experiments [100-102]. Other methods rely on the realization of synthetic magnetic fields by engineering spatially dependent optical couplings $[103,104]$ that lead to Berry phases $[94,105]$ which can be interpreted as the Aharonov-Bohm phase [83] of a charged particle. It has been shown in several proposals that cold atoms in optical lattices are well suited to reach the regime of large magnetic fields on the order of one flux quantum per unit cell by engineering Peierls phases (Sect. 2.1) with the help of laser-assisted tunneling [60,61]. These methods intrinsically lead to effective magnetic fluxes on the order of one flux quantum per unit cell and hence provide direct access to the physics of the Harper-Hofstadter model (Sect. 2.3). Similar ideas led to the development of "optical flux lattices" based on optical dressing in weak periodic potentials [106, 107]. Since then a number of experimental realizations were reported regarding the observation of complex tunneling amplitudes in $1 D[108,109]$ and 2D optical lattices $[67,68]$. Many realizations are based on periodically driven systems that can be mapped onto effective time-independent Hamiltonians (Sect. 3.1) which exhibit the desired physical properties. In particular one can show that simple periodic onsite modulations can give rise to complex tunneling amplitudes (Sect. 3.2). The effective time-independent Hamiltonian however does not provide any information about the full time evolution of the system (Sect. 3.3), which can be of importance for the experimental implementation of artificial magnetic fields in 2D lattices (Sect. 3.4). 


\subsection{Periodically driven quantum systems}

Time-periodic Hamiltonians $\hat{H}(t+T)=\hat{H}(t)$ can be treated using Floquet's theorem, which states that the evolution of the system after one period $T=2 \pi / \omega$ can be described by an effective time-independent Hamiltonian. In most cases it is not possible to find an analytic expression for the effective Floquet Hamiltonian. However, in the high-frequency limit, where $\omega$ is much larger than all other energy scales in the system the effective Hamiltonian can be derived perturbatively. After a short introduction to Floquet theory (Sect. 3.1.1) two different approaches to derive the effective Hamiltonian are presented (Sect. 3.1.2 and 3.1.3), one of them based on the Magnus expansion $[110,111]$ and a second one based on the formalism introduced by Rahav [112]. Eventually both methods are compared using the example of a simple driven two-level system (Sect. 3.1.4).

\subsubsection{Floquet formalism}

According to Floquet theory [113-115] the solutions of the time-dependent Schrödinger equation

$$
i \hbar \frac{\partial}{\partial t}|\Psi(t)\rangle=\hat{H}(t)|\Psi(t)\rangle
$$

associated with a time-periodic Hamiltonian can be written in the following form

$$
\left|\Psi_{\alpha}(t)\right\rangle=\exp \left[-i \epsilon_{\alpha} t / \hbar\right]\left|\Phi_{\alpha}(t)\right\rangle,
$$

where $\left|\Phi_{\alpha}(t)\right\rangle$ is the Floquet mode, which has the same periodicity as the Hamiltonian, $\left|\Phi_{\alpha}(t+T)\right\rangle=\left|\Phi_{\alpha}(t)\right\rangle$. In analogy to Bloch's theorem, $\epsilon_{\alpha}$ is called quasi-energy. By inserting Eq. (3.2) into the time-dependent Schrödinger equation one obtains an eigenvalue equation for the Floquet modes

$$
\left(\hat{H}(t)-i \hbar \frac{\partial}{\partial t}\right)\left|\Phi_{\alpha}(t)\right\rangle=\epsilon_{\alpha}\left|\Phi_{\alpha}(t)\right\rangle \text {. }
$$

One can show that by making the following Fourier expansion

$$
\left|\Phi_{\alpha}(t)\right\rangle=\sum_{\beta} \exp [i \beta \omega t]\left|n_{\alpha}^{\beta}\right\rangle,
$$

the time-dependent problem (3.3) can be transformed into a time-independent one, which involves an infinite matrix [113]. Due to the specific form of the Floquet states, the quasi-energies $\epsilon_{\alpha}$ are only defined up to a multiple of $\hbar \omega$. This can be readily seen by looking at the following expression

$$
\begin{aligned}
\left|\Psi_{\alpha}(t)\right\rangle & =\exp \left[-i\left(\epsilon_{\alpha}+\beta \hbar \omega\right) t / \hbar\right] \exp [i \beta \omega t]\left|\Phi_{\alpha}(t)\right\rangle \\
& =\exp \left[-i \epsilon_{\alpha}^{\beta} t / \hbar\right]\left|\Phi_{\alpha}^{\beta}(t)\right\rangle .
\end{aligned}
$$


Evindently, $\left|\Phi_{\alpha}^{\beta}(t)\right\rangle=\exp [i \beta \omega t]\left|\Phi_{\alpha}(t)\right\rangle$ is the same physical state as $\left|\Phi_{\alpha}(t)\right\rangle$, see also Eq. (3.4). The corresponding quasi-energies are given by $\epsilon_{\alpha}^{\beta}=\epsilon_{\alpha}+\beta \hbar \omega$, where $\beta$ is an integer. Similar to spatially periodic systems, where the quasimomentum is defined within the first Brillouin zone, the quasi-energy can be defined in the range $-\hbar \omega / 2 \leq$ $\epsilon_{\alpha}<\hbar \omega / 2$. The particular structure of the Floquet energy spectrum can have important consequences for the scattering properties of the particles in periodically-driven systems in the presence of interactions [116].

\section{Evolution operator}

Here, we are going to focus on the theoretical description in terms of the unitary timeevolution operator $\hat{U}\left(t, t_{0}\right)$, which evolves a state $\left|\psi\left(t_{0}\right)\right\rangle$ at time $t=t_{0}$ to $|\psi(t)\rangle$ according to

$$
|\psi(t)\rangle=\hat{U}\left(t, t_{0}\right)\left|\psi\left(t_{0}\right)\right\rangle .
$$

The operator $\hat{U}\left(t, t_{0}\right)$ is a solution to the time-dependent Schrödinger equation

$$
i \hbar \frac{\partial}{\partial t} \hat{U}\left(t, t_{0}\right)=\hat{H}(t) \hat{U}\left(t, t_{0}\right), \quad \hat{U}\left(t_{0}, t_{0}\right)=\mathbb{1} .
$$

In general it can be written in the following form

$$
\hat{U}\left(t, t_{0}\right)=\mathcal{T}_{t} \exp \left[-\frac{i}{\hbar} \int_{t_{0}}^{t} \hat{H}\left(t^{\prime}\right) \mathrm{d} t^{\prime}\right],
$$

where $\mathcal{T}_{t}$ is the time-ordering operator, which is a short notation of an infinite series of commutator relations. One can show that for periodic systems the evolution operator fulfills the following properties [117]

$$
\hat{U}(t+T, 0)=\hat{U}(t, 0) \hat{U}(T, 0), \quad \hat{U}(t+T, T)=\hat{U}(t, 0) .
$$

Using these relations one obtains

$$
\hat{U}(n T, 0)=[\hat{U}(T, 0)]^{n}=[\hat{U}(T)]^{n} \text {, with } \hat{U}(T, 0) \equiv \hat{U}(T) \text { and } n \in \mathbb{N} \text {, }
$$

where $\hat{U}(T)$ is the evolution operator over one period $T$. The long-time behavior of the system can be described stroboscopically with $\hat{U}(t)$ at times $t=n T$. This is an essential part of Floquet's theorem [113, 117-120], which tells us that the evolution of the system after multiples of one driving period can be described by an effective time-independent Floquet Hamiltonian $\hat{H}_{F}$

$$
\hat{U}(n T)=[\hat{U}(T)]^{n}=\exp \left[-\frac{i}{\hbar} n T \hat{H}_{F}\right], \quad \hat{U}(T)=\exp \left[-\frac{i}{\hbar} T \hat{H}_{F}\right]
$$


where $\hat{H}_{F}$ is a Hermitian matrix. Note that there exists a whole family of effective Hamiltonians which describe the long-time dynamics of the system. These Hamiltonians are related to each other via gauge transformations; they share the same spectrum and the same topological properties. In general it is not possible to find an analytic expression for $\hat{H}_{F}$. However, in the high frequency limit, where the frequency $\omega=2 \pi / T$ associated with one period $T$ is much faster than all other time-scales of the system, the effective Hamiltonian $\hat{H}_{F}$ can be calculated perturbatively; two different approaches are presented in the following two subsections. Having the Floquet Hamiltonian at hand the theoretical discussion simplifies and the properties of the system can be easily studied with usual techniques that apply for time-independent Hamiltonians. Certainly, this has the disadvantage that information about the evolution within one driving period is lost; this is the so-called micro-motion, which can be important for experiments since it might lead to large oscillations of experimental observables [66, 121, 122]. In a more general form of Floquet's theorem the evolution operator can be partitioned as [112]

$$
\hat{U}\left(t_{f}, t_{i}\right)=\hat{P}\left(t_{f}\right) \mathrm{e}^{-\frac{i}{\hbar} \hat{H}_{F}\left(t_{f}-t_{i}\right)} \hat{P}^{\dagger}\left(t_{i}\right)=\mathrm{e}^{-i \hat{K}\left(t_{f}\right)} \mathrm{e}^{-\frac{i}{\hbar} \hat{H}_{F}\left(t_{f}-t_{i}\right)} \mathrm{e}^{i \hat{K}\left(t_{i}\right)}
$$

where $\hat{P}(t)=\hat{P}(t+T)$ is a time-periodic unitary operator, which was identified in Ref. [121] as initial and final kick-operators; $t_{i}$ is the initial time of the evolution and $t_{f}$ the final one. The validity of Eq. (3.12) can be proven using general properties of the evolution operator

$$
\hat{U}\left(t_{f}, t_{i}\right)=\hat{U}\left(t_{f}, 0\right) \hat{U}\left(0, t_{i}\right)=\hat{U}\left(t_{f}, 0\right) \hat{U}\left(t_{i}, 0\right)^{-1}=\hat{U}\left(t_{f}, 0\right) \hat{U}\left(t_{i}, 0\right)^{\dagger},
$$

together with the well known form of Floquet's theorem [117, 123]

$$
\hat{U}(t, 0)=\hat{P}(t) \mathrm{e}^{-\frac{i}{\hbar} \hat{H}_{F} t}, \quad \hat{P}(0)=\mathbb{1} .
$$

The proof for the time-periodicity of the propagation operator $\hat{P}(t)$ can be found in Ref. [123] and Appendix B.

The general form of the evolution operator (3.12) motivates the following intuitive interpretation. The eigenvalues and eigenstates of the effective time-independent Hamiltonian $\hat{H}_{F}$ are denoted as $\epsilon$ and $|v\rangle$ respectively so that the eigenstates satisfy the following equations

$$
i \hbar \frac{\partial}{\partial t}|v(t)\rangle=\hat{H}_{F}|v(t)\rangle \text {, with }|v(t)\rangle=\mathrm{e}^{-i \epsilon t / \hbar}|v\rangle
$$

Let us further assume that we can find a unitary transformation $\mathrm{e}^{i \hat{K}(t)}$ which maps the system described by the time-periodic Hamiltonian $\hat{H}(t)$ onto a time-independent one governed by $\hat{H}_{F}$. Then the eigenfunctions of the original Hamiltonian are given by

$$
|\Psi(t)\rangle=\mathrm{e}^{-i \hat{K}(t)}|v(t)\rangle=\mathrm{e}^{-i \epsilon t / \hbar} \underbrace{\mathrm{e}^{-i \hat{K}(t)}|v\rangle}_{=|\Phi(t)\rangle}=\mathrm{e}^{-i \epsilon t / \hbar}|\Phi(t)\rangle .
$$


Hence $|\Psi(t)\rangle$ is a solution of the time-periodic Hamiltonian $\hat{H}(t)$ with quasi-energy $\epsilon$ and periodic eigenfunction $|\Phi(t+T)\rangle=|\Phi(t)\rangle$, see also Eqs. (3.1)-(3.3). Consequently, the evolution of the system can be computed by first making a unitary transformation to the time-independent Hamiltonian using the kick-operator $\hat{K}(t)$, then evolving the state according to $\hat{H}_{F}$ for a time $\left(t_{f}-t_{i}\right)$ and at the end transforming it back with $\hat{K}(t)$.

\subsubsection{Mapping to time-independent Hamiltonian}

Following the discussion in the previous section it becomes obvious that one way of solving the time-dependent problem builds on the computation of the unitary transformation $\mathrm{e}^{i \hat{K}(t)}$ that maps the time-dependent Hamiltonian $\hat{H}(t)$ onto a time-independent one $\hat{H}_{F}[112,121]$. We start with the time-dependent Schrödinger equation (3.1). Applying the operator $\mathrm{e}^{i \hat{K}(t)}$ from the left and adding $i \hbar\left[(\partial / \partial t) \mathrm{e}^{i \hat{K}(t)}\right]|\psi(t)\rangle$ leads to the following equation

$$
i \hbar \frac{\partial}{\partial t}\left(\mathrm{e}^{i \hat{K}(t)}|\psi(t)\rangle\right)=\mathrm{e}^{i \hat{K}(t)} \hat{H}(t)|\psi(t)\rangle+i \hbar\left(\frac{\partial}{\partial t} \mathrm{e}^{i \hat{K}(t)}\right)|\psi(t)\rangle,
$$

and together with Eqs. (3.15) and (3.16) we can identify the time-independent Hamiltonian

$$
\hat{H}_{F}=\mathrm{e}^{i \hat{K}(t)} \hat{H}(t) \mathrm{e}^{-i \hat{K}(t)}+i \hbar\left(\frac{\partial}{\partial t} \mathrm{e}^{i \hat{K}(t)}\right) \mathrm{e}^{-i \hat{K}(t)}
$$

In the high-frequency limit the Hamiltonian $\hat{H}_{F}$ and the operator $\hat{K}(t)$ can be expanded in orders of $1 / \omega$

$$
\hat{H}_{F}=\sum_{n=0}^{\infty} \frac{1}{\omega^{n}} \hat{H}_{F}^{(n)} \quad \text { and } \quad \hat{K}=\sum_{n=1}^{\infty} \frac{1}{\omega^{n}} \hat{K}^{(n)} .
$$

The strategy is to compute $\hat{H}_{F}^{(n)}$ as a function of $\hat{K}^{(1)}, \ldots, \hat{K}^{(n+1)}$ and to choose $\hat{K}^{(n+1)}$ iteratively such that $\hat{H}_{F}^{(n)}$ is time-independent. In this way one assures that $\hat{H}_{F}$ is timeindependent in any order $n$. This is achieved using the following operator expansions [112]

$$
\begin{aligned}
& \mathrm{e}^{i \hat{K}(t)} \hat{H}(t) \mathrm{e}^{-i \hat{K}(t)}=\hat{H}+i[\hat{K}, \hat{H}]-\frac{1}{2 !}[\hat{K},[\hat{K}, \hat{H}]]-\frac{i}{3 !}[\hat{K},[\hat{K},[\hat{K}, \hat{H}]]]+\cdots, \\
&\left(\frac{\partial}{\partial t} \mathrm{e}^{i \hat{K}(t)}\right) \mathrm{e}^{-i \hat{K}(t)}=i \frac{\partial \hat{K}}{\partial t}-\frac{1}{2 !}\left[\hat{K}, \frac{\partial \hat{K}}{\partial t}\right]-\frac{i}{3 !}\left[\hat{K},\left[\hat{K}, \frac{\partial \hat{K}}{\partial t}\right]\right]+\cdots
\end{aligned}
$$

\section{Application: Off-resonant driving}

In Ref. [121] the above formalism was applied to periodic Hamiltonians of the following form 


$$
\hat{H}(t)=\hat{H}_{0}+\hat{V}(t)=\hat{H}_{0}+\sum_{j=1}^{\infty}\left(\hat{V}^{(j)} \mathrm{e}^{i j \omega t}+\hat{V}^{(-j)} \mathrm{e}^{-i j \omega t}\right),
$$

where the driving frequency $\omega$ is much larger than all other energy scales of the system $\omega \rightarrow \infty$. Making use of the operator expansions (3.20) and (3.21) one can derive general expressions for the time-independent Hamiltonian $\hat{H}_{F}$ and the kick-operator $\hat{K}(t)$

$$
\begin{aligned}
& \hat{H}_{F}=\hat{H}_{0}+\frac{1}{\hbar \omega} \sum_{j=1}^{\infty} \frac{1}{j}\left[\hat{V}^{(j)}, \hat{V}^{(-j)}\right] \\
&+\frac{1}{2(\hbar \omega)^{2}} \sum_{j=1}^{\infty} \frac{1}{j^{2}}\left(\left[\left[\hat{V}^{(j)}, \hat{H}_{0}\right], \hat{V}^{(-j)}\right]+\left[\left[\hat{V}^{(-j)}, \hat{H}_{0}\right], \hat{V}^{(j)}\right]\right)+\mathcal{O}\left(\frac{1}{\omega^{3}}\right) \\
& \hat{K}(t)=\frac{1}{\hbar} \int^{t} \hat{V}(\tau) \mathrm{d} \tau+\mathcal{O}\left(\frac{1}{\omega^{2}}\right)=\sum_{j \neq 0} \frac{1}{i j \hbar \omega} \hat{V} \mathrm{e}^{i j \omega t}+\mathcal{O}\left(\frac{1}{\omega^{2}}\right) .
\end{aligned}
$$

A more complete expression including higher order terms as well as second order terms that mix different harmonics can be found in Ref. [121]. This formalism applies for example to periodically shaken lattices as realized experimentally in Refs. [54, 67, 109, 124, 125].

\section{Application: resonant driving}

Recently, the formalism described above was extended to resonant driving [66], where the driving frequency $\omega$ is still large but resonant with an energy scale of the static part of the Hamiltonian $\hat{H}_{0}$, which is the case in the experimental setups of Refs. [68-73, 126]. The effective Hamiltonian in Eq. (3.23) was calculated using the perturbative expansion in powers of $1 / \omega(3.19)$. This derivation is based on the assumption that the periodic Hamiltonian $\hat{H}(t)$ remains finite in the limit $\omega \rightarrow \infty$. For the Hamiltonians considered here, however, the static Hamiltonian contains a term which is proportional to $\hbar \omega$, hence it diverges in the limit $\omega \rightarrow \infty$. We write the static Hamiltonian in the following form

$$
\hat{H}_{0}=\sum_{\alpha \beta} \hat{P}^{\alpha} \hat{H}_{\alpha \beta}^{(0)} \hat{P}^{\beta}+\hbar \omega \sum_{\alpha} \alpha \hat{P}^{\alpha}, \quad \alpha, \beta \in \mathbb{Z},
$$

where $\hat{P}^{\alpha}$ is a projection operator, which divides the full Hilbert space into a set of orthogonal sectors $\left(\hat{P}^{\alpha} \hat{P}^{\beta}=\delta_{\alpha \beta} \hat{P}^{\alpha}\right)$ labelled by the integer $\alpha$, and $\sum_{\alpha} \hat{P}^{\alpha}=\mathbb{1}$. The number of sectors $\alpha$ depends on the specific problem. Later in this chapter we are going to present simple examples such as the dynamics of a particle hopping on a superlattice potential (Fig. 3.1a) in the presence of periodic driving (Sect. 3.3). In this example the potential energy is increased on every other site by an amount $\hbar \omega$ and the Hilbert space separates into two parts $\alpha=\{0,1\}$. In general, terms of the form $\hat{H}_{\alpha \beta}(\alpha \neq \beta)$ couple different sectors, while the terms $\hat{H}_{\alpha \alpha}$ describe additional diagonal terms such as on-site 
interactions or on-site potentials. We assume that all divergent terms of the Hamiltonian are contained in the term $\hbar \omega \sum_{\alpha} \alpha \hat{P}^{\alpha}$ and all remaining components do not consist of terms that diverge with $\omega$. The time-periodic part is consistently written as

$$
\hat{V}(t)=\sum_{j>0}\left[\hat{V}^{(j)} \mathrm{e}^{i j \omega t}+\hat{V}^{(-j)} \mathrm{e}^{-i j \omega t}\right], \quad \hat{V}^{(j)}=\sum_{\alpha \beta} \hat{P}^{\alpha} \hat{H}_{\alpha \beta}^{(j)} \hat{P}^{\beta}
$$

We start by applying a time-dependent unitary transformation to the Hamiltonian $\hat{H}(t)=$ $\hat{H}_{0}+\hat{V}(t)$ according to

$$
|\psi(t)\rangle \rightarrow\left|\psi^{\prime}(t)\right\rangle=\hat{R}(t)|\psi(t)\rangle, \quad \text { with } \quad \hat{R}(t)=\exp \left[i \sum_{\alpha} \alpha \omega t \hat{P}^{\alpha}\right] .
$$

Hence, the new Hamiltonian $\hat{\mathcal{H}}(t)$ takes the following form

$$
\begin{aligned}
& \hat{\mathcal{H}}(t)=\hat{R} \hat{H}(t) \hat{R}^{\dagger}-i \hbar \hat{R} \mathrm{~d}_{t} \hat{R}^{\dagger}=\sum_{j} \hat{\mathcal{H}}(j) \mathrm{e}^{i j \omega t}, \\
& \hat{\mathcal{H}}(j)=\sum_{\alpha \beta} \hat{P}^{\alpha} \hat{H}_{\alpha \beta}^{(j-\alpha+\beta)} \hat{P}^{\beta} .
\end{aligned}
$$

The transformed Hamiltonian does not contain any divergent terms proportional to $\omega$ and is periodic in time. Moreover it can be recast into the form of Hamiltonian (3.22), which was studied in Ref. [121], such that the formalism described above can be directly applied. The time-evolution operator now reads

$$
\hat{U}\left(t_{f}, t_{i}\right)=\hat{R}^{\dagger}\left(t_{f}\right) \mathrm{e}^{-i \hat{\mathcal{K}}\left(t_{f}\right)} \mathrm{e}^{-\frac{i}{\hbar} \hat{\mathcal{H}}_{F}\left(t_{f}-t_{i}\right)} \mathrm{e}^{i \hat{\mathcal{K}}\left(t_{i}\right)} \hat{R}\left(t_{i}\right),
$$

which is essentially the evolution operator given in Eq. (3.12) accompanied by the unitary transformation used to treat the divergent terms of the static Hamiltonian $\hat{H}_{0}$ (3.25). The effective time-independent Hamiltonian $\hat{\mathcal{H}}_{F}$ and the kick-operator $\hat{\mathcal{K}}(t)$ can be computed using analogue expressions as given in Eqs. (3.23) and (3.24)

$$
\begin{aligned}
\hat{\mathcal{H}}_{F} & =\hat{\mathcal{H}}^{(0)}+\frac{1}{\hbar \omega} \sum_{j>0} \frac{1}{j}\left[\hat{\mathcal{H}}^{(+j)}, \hat{\mathcal{H}}^{(-j)}\right]+\mathcal{O}\left(\frac{1}{\omega^{2}}\right), \\
\hat{\mathcal{K}}(t) & =\frac{1}{i \hbar \omega} \sum_{j>0}\left[\hat{\mathcal{H}}^{(+j)} \mathrm{e}^{i j \omega t}-\hat{\mathcal{H}}^{(-j)} \mathrm{e}^{-i j \omega t}\right]+\mathcal{O}\left(\frac{1}{\omega^{2}}\right) .
\end{aligned}
$$

The full time-evolution operator can be written as

$$
\hat{\mathcal{U}}\left(t_{f}, t_{i}\right)=\mathrm{e}^{-i \hat{\mathcal{M}}\left(t_{f}\right)} \mathrm{e}^{-\frac{i}{\hbar} \hat{\mathcal{H}}_{F}\left(t_{f}-t_{i}\right)} \mathrm{e}^{i \hat{\mathcal{M}}\left(t_{i}\right)}, \quad \mathrm{e}^{i \hat{\mathcal{M}}(t)} \equiv \mathrm{e}^{i \hat{\mathcal{K}}(t)} \hat{R}(t),
$$

where from now on we refer to $\hat{\mathcal{M}}(t)$ as the micro-motion operator. 


\subsubsection{Magnus-expansion approach}

The perturbative approach presented above is very powerful in computing the full time evolution of periodic Hamiltonians. In this section we are going to compare this approach to a different perturbative treatment based on the Magnus expansion $[110,111,127]$. This method is particularly useful for describing the stroboscopic longtime evolution of the system at times $t=n T$, with $n \in \mathbb{N}$. According to Floquet theory this evolution can be described by an effective time-independent Floquet Hamiltonian $\hat{H}_{F}$ as given in Eq. (3.11). In the high-frequency limit $\omega \rightarrow \infty$ the Floquet Hamiltonian can be computed in a perturbative manner using the Magnus expansion [110, 111]

$$
\hat{H}_{F}=\sum_{n=0}^{\infty} \hat{H}_{F}^{(n)}
$$

where the lowest two orders are given by

$$
\begin{aligned}
\hat{H}_{F}^{(0)} & =\frac{1}{T} \int_{0}^{T} \hat{H}(t) \mathrm{d} t \\
\hat{H}_{F}^{(1)} & =\frac{-i}{2 \hbar T} \int_{0}^{T} \int_{0}^{t_{2}}\left[\hat{H}\left(t_{2}\right), \hat{H}\left(t_{1}\right)\right] \mathrm{d} t_{1} \mathrm{~d} t_{2} .
\end{aligned}
$$

Higher orders of the Magnus expansion scale as $1 / \omega^{n}$, such that in the high frequency limit the effective time-independent Hamiltonian is well approximated by the lowest order of the expansion $\hat{H}_{F} \simeq \hat{H}_{F}^{(0)}$. The explicit form of the effective time-averaged Hamiltonian depends on the time interval chosen for the integration, $[0, T]$ and $\left[t_{0}, t_{0}+T\right]$ respectively. This gauge freedom is also known as Floquet gauge freedom [127]. In the following we consider Hamiltonians of the form $\hat{H}(t)=\hat{H}_{0}+\hat{V}(t)$ as defined in Eqs. (3.25) and (3.26). The static term of the Hamiltonian contains diverging components proportional to $\omega$. Similar to the method described above we perform a unitary transformation. However, this time it further involves the time-periodic part of the Hamiltonian $\hat{V}(t)$ $[127,128]$ and is defined according to

$$
\left|\psi_{M}(t)\right\rangle=\hat{R}_{M}(t)|\psi(t)\rangle, \text { with } \quad \hat{R}_{M}(t)=\exp \left[i \sum_{\alpha} \alpha \omega t \hat{P}^{\alpha}+i \frac{1}{\hbar} \int^{t} \hat{V}\left(t^{\prime}\right) \mathrm{d} t^{\prime}\right] .
$$

This transformation leads to the new Hamiltonian

$$
\hat{\mathcal{H}}_{M}(t)=\hat{R}_{M} \hat{H}(t) \hat{R}_{M}^{+}-i \hbar \hat{R}_{M} \mathrm{~d}_{t} \hat{R}_{M}^{+} .
$$

The Hamiltonian in the rotating frame $\hat{\mathcal{H}}_{M}(t)$ is time-periodic so that in general the full time-evolution operator can be partitioned as

$$
\hat{U}\left(t_{f}, t_{i}\right)=\hat{R}_{M}^{+}\left(t_{f}\right) \mathrm{e}^{-i \hat{\mathcal{K}}_{M}\left(t_{f}\right)} \mathrm{e}^{-\frac{i}{\hbar} \hat{\mathcal{H}}_{F}^{M}\left(t_{f}-t_{i}\right)} \mathrm{e}^{i \hat{\mathcal{K}}_{M}\left(t_{i}\right)} \hat{R}_{M}\left(t_{i}\right)
$$


with the corresponding micro-motion operator

$$
\mathrm{e}^{i \hat{\mathcal{M}}_{M}(t)} \equiv \mathrm{e}^{i \hat{\mathcal{K}}_{M}(t)} \hat{R}_{M}(t) .
$$

In a stroboscopic analysis the lowest order of the Floquet Hamiltonian $\hat{\mathcal{H}}_{F}^{M}$ in the rotating frame can be computed using the Magnus expansion in Eq. (3.35)

$$
\hat{\mathcal{H}}_{F}^{M} \simeq \frac{1}{T} \int_{0}^{T} \hat{\mathcal{H}}_{M}(t) \mathrm{d} t
$$

In this case the stroboscopic evolution of the system in the lab-frame is determined by

$$
\hat{U}(t, 0)=\hat{R}_{M}^{\dagger}(t) \mathrm{e}^{-\frac{i}{\hbar} \hat{\mathcal{H}}_{F}^{M} t} \hat{R}_{M}(0)
$$

where $t=n T, n \in \mathbb{N}$.

\subsubsection{Comparison of the two methods: Two-level system}

The two perturbative methods presented above are compared based on a two-level system with levels $|0\rangle$ and $|1\rangle$, that are separated by a large energy offset $\Delta$. The system is described by the static Hamiltonian

$$
\hat{H}=J(|0\rangle\langle 1|+| 1\rangle\langle 0|)+\Delta \hat{P}^{1}, \quad \hat{P}^{1}=|1\rangle\langle 1|,
$$

where $J$ is the coupling between the two levels. The energy difference between the two eigenstates is given by

$$
E_{\text {gap }}=\sqrt{\Delta^{2}+4 J^{2}}
$$

This sets the exact value for the driving frequency to couple the two levels resonantly $\hbar \omega=E_{\text {gap }}$. In the limit of a large energy offset $\Delta \gg J$, this difference is approximately given by $E_{\text {gap }} \simeq \Delta$ with the resonance condition $\hbar \omega \simeq \Delta$. The dynamics of the system in the presence of a resonant periodic modulation with frequency $\omega$ is described by the following time-periodic Hamiltonian

$$
\hat{H}(t)=J(|0\rangle\langle 1|+| 1\rangle\langle 0|)+\hbar \omega \hat{P}^{1}+V_{0} \cos (\omega t+\varphi) \hat{P}^{0}, \quad \hat{P}^{\alpha}=|\alpha\rangle\langle\alpha|,
$$

with $\hat{P}^{\alpha}$ the projectors on the two sectors $\alpha=\{0,1\}$ and $V_{0}$ the modulation amplitude. Following the method presented in Sect. 3.1 .3 we perform a unitary transformation into the rotating frame $[127,128]$ as given in Eqs. (3.37) and (3.38) with

$$
\hat{R}_{M}(t)=\exp \left[i \omega t \hat{P}^{1}+i \frac{V_{0}}{\hbar \omega} \sin (\omega t+\varphi) \hat{P}^{0}\right] .
$$

The only component in the Hamiltonian which is affected by this transformation is the coupling term proportional to $J$, which results in a time-dependent coupling 


$$
\begin{aligned}
& \hat{\mathcal{H}}_{M}(t)=J|0\rangle\left\langle 1\left|\mathrm{e}^{i \eta(t)}+J\right| 1\right\rangle\langle 0| \mathrm{e}^{-i \eta(t)}, \\
& \eta(t)=-\left[\omega t-\frac{V_{0}}{\hbar \omega} \sin (\omega t+\varphi)\right] .
\end{aligned}
$$

The transformed Hamiltonian $\hat{\mathcal{H}}_{M}(t)$ is periodic in time without any static components. To lowest order the effective Hamiltonian is determined by Eq. (3.41)

$$
\begin{aligned}
\hat{\mathcal{H}}_{F}^{M} & =\frac{1}{T} \int_{0}^{T} \hat{\mathcal{H}}_{M}(t) \mathrm{d} t \\
& =\frac{1}{2 \pi} \int_{0}^{2 \pi}\left[J|0\rangle\langle 1| \mathrm{e}^{-i\left[\tau-\frac{V_{0}}{\hbar \omega} \sin (\tau+\varphi)\right]}+\text { h.c. }\right] \mathrm{d} \tau \\
& =\underbrace{J \mathcal{J}_{1}\left(\frac{V_{0}}{\hbar \omega}\right)}_{=: J_{\text {eff }}} \mathrm{e}^{i \varphi}|0\rangle\langle 1|+\text { h.c., }
\end{aligned}
$$

where $\tau=\omega t$ and $\mathcal{J}_{1}(x)=\frac{1}{2 \pi} \int_{0}^{2 \pi} \mathrm{e}^{-i(\tau-x \sin \tau)} \mathrm{d} \tau$ is the first-order Bessel function of the first kind. The coupling between the two levels $|0\rangle$ and $|1\rangle$ is restored in the presence of resonant driving Jeff $=J \mathcal{J}_{1}\left(V_{0} /[\hbar \omega]\right) \simeq J V_{0} /(2 \hbar \omega)$ and is accompanied by an additional complex term $\mathrm{e}^{i \varphi}$, which can be used to generate artificial magnetic fields as will be shown in the following sections.

The micro-motion operator $\hat{\mathcal{K}}_{M}(t)$ associated with the Hamiltonian $\hat{\mathcal{H}}_{M}(t)$ can be calculated perturbatively in the high-frequency limit $\hbar \omega \gg J[110,111,129]$. The two lowest orders of the expansion are given by

$$
\begin{aligned}
\mathrm{e}^{-i \hat{\mathcal{K}}_{M}(t)} & =\mathbb{1}-\frac{i}{\hbar} \int_{0}^{t} \hat{\mathcal{H}}_{M}\left(t^{\prime}\right) \mathrm{d} t^{\prime}+\cdots \\
& \simeq \mathbb{1}-\underbrace{\frac{i J}{\hbar \omega} \int_{0}^{\tau}\left[|0\rangle\langle 1| \mathrm{e}^{-i\left[\tau^{\prime}-\frac{V_{0}}{\hbar \omega} \sin \left(\tau^{\prime}+\varphi\right)\right]}+\text { h.c. }\right] \mathrm{d} \tau^{\prime}}_{\rightarrow 0 \text { for } \omega \rightarrow \infty} .
\end{aligned}
$$

Consequently, in the limit of infinite driving frequencies $\omega \rightarrow \infty$ the full-time dynamics associated with the time-periodic Hamiltonian $\hat{\mathcal{H}}_{M}(t)$ in the rotating frame (3.47) is exactly described by the effective time-independent Hamiltonian $\hat{\mathcal{H}}_{F}^{M}$ (3.48). Hence, the full time evolution of the driven two-level system is approximately given by Eq. (3.42) for arbitrary times $t \neq n T$, with $n \in \mathbb{N}$, and the micro-motion in the lab-frame is solely determined by the unitary transformation $\hat{R}_{M}(t)$ (3.46). Note, however, that this is not true in general.

The same system is now analyzed again using the formalism developed in [66] as discussed in Sect. 3.1.2. The Hamiltonian describing the two-level system (3.45) is indeed of the form (3.25)-(3.26) and we perform the unitary transformation to remove the diverging terms of the Hamiltonian as defined in Eqs. (3.27)-(3.29) using the unitary operator 


$$
\hat{R}(t)=\exp \left[i \omega t \hat{P}^{1}\right] .
$$

Note that this operator differs from the transformation used for the Magnus-expansion approach (3.46) and contains only the static diverging terms of $\hat{H}_{0}$. The new Hamiltonian reads

$$
\begin{aligned}
& \hat{\mathcal{H}}(t)=\hat{\mathcal{H}}^{(+1)} \mathrm{e}^{i \omega t}+\hat{\mathcal{H}}^{(-1)} \mathrm{e}^{-i \omega t}, \\
& \hat{\mathcal{H}}^{(+1)}=J|1\rangle\langle 0|+\frac{V_{0}}{2} \hat{P}^{0} \mathrm{e}^{i \varphi}, \\
& \hat{\mathcal{H}}^{(-1)}=J|0\rangle\langle 1|+\frac{V_{0}}{2} \hat{P}^{0} \mathrm{e}^{-i \varphi} .
\end{aligned}
$$

Using Eq. (3.31) we can derive the effective time-independent Hamiltonian

$$
\begin{aligned}
\hat{\mathcal{H}}_{F} & =\frac{1}{\hbar \omega}\left[\hat{\mathcal{H}}^{(+1)}, \hat{\mathcal{H}}^{(-1)}\right] \\
& =\frac{-1}{\hbar \omega}(\underbrace{[J|0\rangle\langle 1|, J| 1\rangle\langle 0|]}_{=J^{2}\left[\hat{P}^{0}-\hat{P}^{1}\right]}+\left[\frac{V_{0}}{2} \hat{P}^{0} \mathrm{e}^{-i \varphi}, J|1\rangle\langle 0|\right]+\left[J|0\rangle\langle 1|, \frac{V_{0}}{2} \hat{P}^{0} \mathrm{e}^{i \varphi}\right]) \\
& \simeq \frac{J V_{0}}{2 \hbar \omega}\left(|0\rangle\left\langle 1\left|\mathrm{e}^{i \varphi}+\right| 1\right\rangle\langle 0| \mathrm{e}^{-i \varphi}\right),
\end{aligned}
$$

where we have omitted the additional detuning term

$$
\hat{\mathcal{H}}_{\text {det }}=-\frac{J^{2}}{\hbar \omega}\left[\hat{P}^{0}-\hat{P}^{1}\right]
$$

This term also appears in the second order of the Magnus expansion [127]. For typical experimental parameters used for the measurements discussed in the context of this thesis, $\hbar \omega / J=\Delta / J \simeq 30$, and the detuning can be safely neglected. One exception where this assumption does not hold anymore is presented in Sect. 5.7 where the ratio is about one order of magnitude smaller $\Delta / J \simeq 3.56$.

The effective Hamiltonian (3.52) is in agreement with the result obtained using the Magnus expansion (3.48) in the limit $V_{0} /(\hbar \omega) \ll 1$. The main contribution to the kickoperator according to Eq. (3.32) is given by

$$
\begin{aligned}
\hat{\mathcal{K}}(t) & =\frac{1}{i \hbar \omega}\left[J|1\rangle\langle 0|+\frac{V_{0}}{2} \hat{P}^{0} \mathrm{e}^{i \varphi}\right] \mathrm{e}^{i \omega t}-\frac{1}{i \hbar \omega}\left[J|0\rangle\langle 1|+\frac{V_{0}}{2} \hat{P}^{0} \mathrm{e}^{-i \varphi}\right] \mathrm{e}^{-i \omega t} \\
& \simeq \frac{V_{0}}{\hbar \omega} \sin (\omega t+\varphi) \hat{P}^{0}
\end{aligned}
$$

where we considered again the limit $J \ll \hbar \omega$. As a result the main contributions to the micro-motion operator are

$$
\hat{\mathcal{M}}(t)=\omega t \hat{P}^{1}+\frac{V_{0}}{\hbar \omega} \sin (\omega t+\varphi) \hat{P}^{0},
$$

which is in agreement with the Magnus-expansion approach discussed above. 


\subsection{Peierls phases and multi-photon processes}

For the remaining part of this chapter we restrict our discussion to the perturbative treatment of time-periodic Hamiltonians based on the Magnus expansion. For simplicity we omit the index $M$ and denote the Floquet Hamiltonian in the rotating frame as $\hat{\mathcal{H}}_{F}^{M} \equiv \hat{\mathcal{H}}_{F}$. The theoretical description of the two-level system can be easily extended to more general situations sometimes called multi-photon processes where an integer multiple of the driving frequency $\omega$ is used to restore resonant tunneling between the two levels $|0\rangle$ and $|1\rangle[130,131]$. Let us consider the following periodically driven twolevel system described by the Hamiltonian

$$
\hat{H}(t)=J(|0\rangle\langle 1|+| 1\rangle\langle 0|)+\Delta \hat{P}^{1}+V_{0} \cos (\omega t+\varphi) \hat{P}^{0},
$$

where $\Delta$ is the energy difference between the two levels. For resonant driving $\Delta=v \hbar \omega$, with $v \in \mathbb{Z}$, the effective time-independent Hamiltonian takes a very simple form using the integral representation of the Bessel functions of the first kind $\mathcal{J}_{v}(x)=$ $\frac{1}{2 \pi} \int_{0}^{2 \pi} \mathrm{e}^{i(v \tau-x \sin \tau)} \mathrm{d} \tau$

$$
\hat{\mathcal{H}}_{F}=J \mathcal{J}_{v}\left(\frac{V_{0}}{\hbar \omega}\right)\left[\mathrm{e}^{i v \varphi}|0\rangle\left\langle 1\left|+\mathrm{e}^{-i v \varphi}\right| 1\right\rangle\langle 0|\right]
$$

In the limit $\omega \rightarrow \infty$ this Hamiltonian is exact for the dynamics described in the rotating frame as shown in Sect. 3.1.4. The phase factor $v \varphi$ scales with the order $v$ of the multi-photon process. In the context of this thesis we consider zero-order $v=0$ as well as first-order $v= \pm 1$ processes. Zero-order processes do not lead to complex couplings but the strength of the tunnel coupling is renormalized according to the zeroth order Bessel function $\mathcal{J}_{0}\left(V_{0} /[\hbar \omega]\right)$, which was observed experimentally in Ref. [124]. Firstorder processes on the other hand lead to complex tunneling matrix elements; these processes are typically referred to as laser-assisted tunneling. Note that there is an important difference regarding the sign of the energy offset $\Delta$ or driving frequency $\omega$, which is summarized in the table below.

\begin{tabular}{|c|c|c|}
\hline Resonance condition & Effective coupling strength & Phase factor \\
\hline$\hbar \omega=\Delta$ & $J \mathcal{J}_{1}\left(V_{0} /[\hbar \omega]\right)$ & $\varphi$ \\
$\hbar \omega=-\Delta$ & $J \mathcal{J}_{1}\left(V_{0} /[\hbar \omega]\right)$ & $-\varphi+\pi$ \\
\hline
\end{tabular}

The effective coupling strength induced by the laser-assisted tunneling is independent of the sign in the resonance condition $\hbar \omega= \pm \Delta$, the phase factor, however, does depend on it. In particular, the sign of the phase changes, which will be important for the experimental realization of different flux distributions as discussed in chapter 5-7. 
a

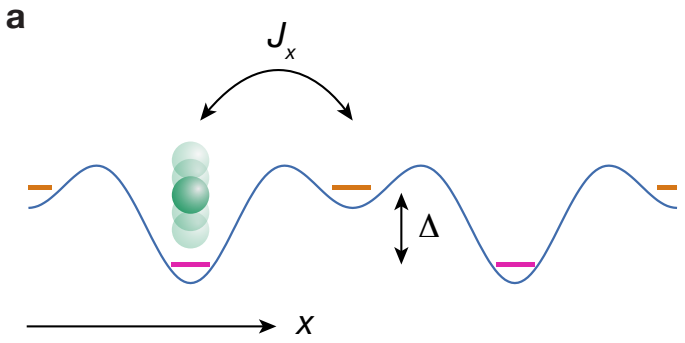

b

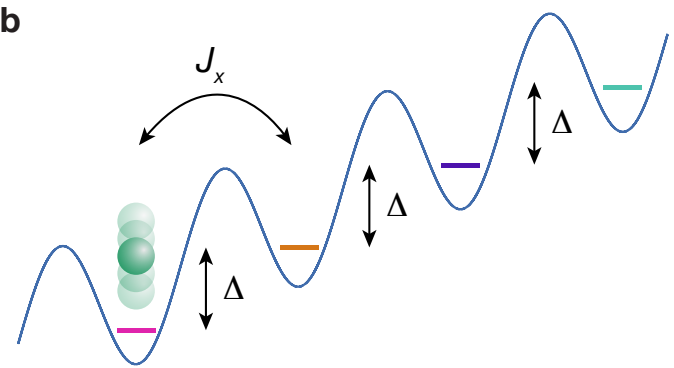

Figure 3.1: Schematic drawings of the periodically modulated one-dimensional lattice potentials. a Superlattice potential with two non-equivalent sites $(\alpha=\{0,1\})$ with energy offsets $\Delta_{m}=(-1)^{m} \Delta / 2$. $\mathbf{b}$ Wannier-Stark ladder with linearly increasing on-site energy $\Delta_{m}=m \Delta$ $(\alpha=\{0,1,2,3, \ldots\})$. The different colors refer to different sectors $\alpha$. In both cases the energy offset between neighboring sites is $\Delta$ which inhibits tunneling along the $x$-direction for $\Delta \gg J_{x}$, with bare tunnel coupling $J_{x}$. Resonant tunneling can be restored in both cases with a singleharmonic driving at frequency $\hbar \omega=\Delta$. (Figure adapted from Ref. [66])

\subsection{Periodically driven one-dimensional lattices}

The description of a time-periodic system in terms of a time-independent Floquet Hamiltonian $\hat{\mathcal{H}}_{F}$ might not always be sufficient since the evolution within one period might give rise to large oscillations of experimental observables. In this section we discuss the micro-motion $\hat{\mathcal{M}}(t)$ within one Floquet period for periodically modulated onedimensional lattice potentials using two examples, which are illustrated in Fig. 3.1. We consider the dynamics of atoms in a one-dimensional lattice potential with additional site-dependent energy offsets $\Delta_{m}$ described by the following tight-binding Hamiltonian

$$
\hat{H}(t)=-J_{x} \sum_{m}\left(\hat{a}_{m+1}^{\dagger} \hat{a}_{m}+\text { h.c. }\right)+\sum_{m}\left(V_{0} \sin \left(\omega t+\varphi_{m}\right)+\Delta_{m}\right) \hat{n}_{m},
$$

where $\varphi_{m}$ is the site-dependent phase of the driving. In the context of this thesis only potentials of the form $\Delta_{m+1}-\Delta_{m}= \pm \Delta= \pm \hbar \omega$ are considered but the model can be extended to other potentials where the energy offset between neighboring sites is an integer multiple of $\Delta$ as demonstrated in Ref. [66]. The Hamiltonian in Eq. (3.58) is periodic in time $\hat{H}(t+T)=\hat{H}(t)$ and is indeed of the form given in Eqs. (3.25) and (3.26). The static divergent term of the Hamiltonian can be written in the following form

$$
\hat{S}=\sum_{m} \Delta_{m} \hat{n}_{m}=\hbar \omega \sum_{\alpha} \alpha \hat{N}_{\alpha}, \quad \hat{N}_{\alpha}=\sum_{m \in \alpha} \hat{n}_{m}
$$

where $\hat{N}_{\alpha}$ is the number operator for the sector $\alpha$ and $\alpha \in \mathbb{Z}$. The sites $m$ that belong to one sector $\alpha$ are defined by the on-site potential $\Delta_{m}$. For the potential illustrated in Fig. 3.1a there are two different sectors $\alpha=\{0,1\}$, where every other site belongs to the same sector. In the case of the Wannier-Stark ladder depicted in Fig. 3.1b, the number 
of sectors is infinite and each site belongs to a different sector. The number operator $\hat{N}_{\alpha}$ on the other hand can be written in terms of projectors

$$
\hat{N}_{\alpha}=\sum_{n_{\alpha}} n_{\alpha} \hat{P}_{n_{\alpha}}, \quad \hat{N}_{\alpha}\left|n_{\alpha}\right\rangle=n_{\alpha}\left|n_{\alpha}\right\rangle .
$$

Using this expression together with Eq. (3.59) we obtain

$$
\hat{S}=\hbar \omega \sum_{\alpha, n_{\alpha}}\left(\alpha n_{\alpha}\right) \hat{P}_{n_{\alpha}}
$$

We start by performing a unitary transformation to the rotating frame as defined in Eqs. (3.37)-(3.38) using the unitary operator

$$
\hat{R}(t)=\exp \left[i \sum_{m}\left(-\frac{V_{0}}{\hbar \omega} \cos \left(\omega t+\varphi_{m}\right)+\frac{\Delta_{m} t}{\hbar}\right) \hat{n}_{m}\right]=\exp \left[i \sum_{m} \chi_{m}(t) \hat{n}_{m}\right],
$$

which leads to the new transformed Hamiltonian

$$
\hat{\mathcal{H}}(t)=-J_{x} \sum_{m}\left(\mathrm{e}^{i \eta_{m}(t)} \hat{a}_{m+1}^{\dagger} \hat{a}_{m}+\text { h.c. }\right),
$$

with $\eta_{m}(t)=\chi_{m+1}(t)-\chi_{m}(t)$ determined by the following equations

$$
\begin{aligned}
\eta_{m}(t) & =\left[-\cos \left(\omega t+\varphi_{m+1}\right)+\cos \left(\omega t+\varphi_{m}\right)\right] V_{0} /(\hbar \omega)+\left(\Delta_{m+1}-\Delta_{m}\right) t / \hbar \\
& =-\frac{2 V_{0}}{\hbar \omega} \sin \left(\omega t+\frac{\varphi_{m+1}+\varphi_{m}}{2}\right) \sin \left(\frac{\varphi_{m+1}-\varphi_{m}}{2}\right)+\left(\Delta_{m+1}-\Delta_{m}\right) t / \hbar \\
& =:-\eta_{0} \sin \left(\omega t+\frac{\varphi_{m+1}+\varphi_{m}}{2}\right)+\left(\Delta_{m+1}-\Delta_{m}\right) t / \hbar .
\end{aligned}
$$

Using the equations above we study the micro-motion for different on-site potentials $\Delta_{m}$, which is fully determined by the unitary operator $\hat{R}(t)$ in Eq. (3.62) as shown above (Sect. 3.1.4).

\subsubsection{Micro-motion staggered superlattice potential}

The staggered superlattice potential illustrated in Fig. 3.1a consists of two non-equivalent lattice sites (two sectors $\alpha$ ) with on-site potential energies that alternate in sign along the direction of the staggered potential $\Delta_{m}=(-1)^{m} \Delta / 2$. Hence, the micro-motion operator is determined by

$$
\hat{\mathcal{M}}_{\text {stagg }}(t)=\sum_{m}\left(-\frac{V_{0}}{\hbar \omega} \cos \left(\omega t+\varphi_{m}\right)+\frac{\Delta t}{2 \hbar}(-1)^{m}\right) \hat{n}_{m} .
$$

First of all we note that $\hat{\mathcal{M}}(t)$ is proportional to the number operator $\hat{n}_{m}$. Consequently, the density distribution of the wave function is not affected by the micro-motion contrary 
to the quasimomentum distribution. With non-interacting bosonic atoms this distribution can be probed using standard time-of-flight (TOF) imaging (see also Sect. 5.2) [29]. The micro-motion operator consists of two distinct parts:

$$
\hat{\mathcal{M}}(t)_{\bmod }=\sum_{m}\left(-\frac{V_{0}}{\hbar \omega} \cos \left(\omega t+\varphi_{m}\right)\right) \hat{n}_{m}
$$

and

$$
\hat{\mathcal{M}}(t)_{\text {pot }}=\sum_{m}\left(\frac{\Delta t}{2 \hbar}(-1)^{m}\right) \hat{n}_{m}
$$

The first one is associated with the periodic on-site modulation and is proportional to $\gamma=V_{0} /(\hbar \omega)$. To gain more insight into the properties of this operator we study the evolution of a state $|G S\rangle$ under the action of $\hat{\mathcal{M}}(t)_{\bmod }$,

$$
|\psi(t)\rangle=\exp \left[i \hat{\mathcal{M}}(t)_{\mathrm{mod}}\right]|G S\rangle
$$

The initial state at $t=0$ is chosen as the ground state $|G S\rangle$ of the normal lattice without periodic driving $\left(V_{0}=0\right)$ and without potential energy offset between neighboring sites $(\Delta=0)$. This state exhibits a single quasimomentum component at zero momentum (black solid line in Fig. 3.2). For $V_{0}>0$ the operator $\hat{\mathcal{M}}(t)_{\bmod }$ generally gives rise to additional momentum components. For a linear phase distribution $\varphi_{m}=2 \pi \alpha m$ these components are separated by multiples of $\delta k=2 \pi \alpha / a$, where $a$ is the lattice constant and the position in the lattice is defined as $\mathbf{R}=m a \hat{\mathbf{e}}_{x}$, with $\hat{\mathbf{e}}_{x}$ the unit vector along $x$ [61]. In Figure 3.2 the quasimomentum distribution is plotted for $\alpha=1 / 4$, different values of the modulation amplitude $V_{0}$ and different evolution times $t$. As expected additional momentum components appear in the distribution at period $\delta k=\pi /(2 a)$. The amplitudes of the individual components oscillate periodically with period $T \alpha$, but their positions remain unchanged.

The second part $\hat{\mathcal{M}}(t)_{\text {pot }}$ is determined by the potential which inhibits bare tunneling for $\Delta \gg J_{x}$. We perform similar calculations as before but this time we act with $\hat{\mathcal{M}}(t)_{\text {pot }}$ on the ground state $|G S\rangle$ of the normal lattice

$$
|\psi(t)\rangle=\exp \left[i \hat{\mathcal{M}}(t)_{\mathrm{pot}}\right]|G S\rangle
$$

The quasimomentum distribution of $|\psi(t)\rangle$ features oscillations between the momentum components at zero and $\pm \pi / a$ with an oscillation period $T=h / \Delta$ (Fig. 3.3a). The evolution starts at zero momentum for $t=0$. After half an oscillation $t=T / 2$ the component at zero momentum disappears and the distribution shows only components at the edge of the Brillouin zone $( \pm \pi / a)$. For longer times the amplitudes of the different momentum components oscillate but no additional components are populated. 

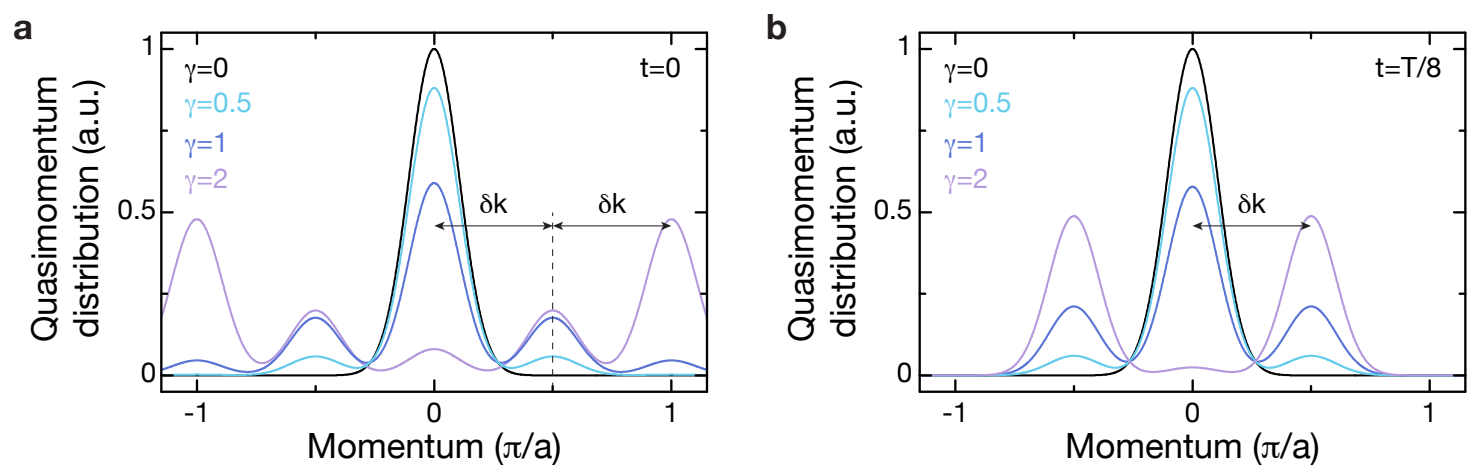

Figure 3.2: Typical momentum distribution after acting with the modulation-induced micromotion operator $\hat{\mathcal{M}}(t)_{\text {mod }}$ on an initial state with zero quasimomentum. The initial state is the ground state of the unperturbed lattice potential with lattice constant $a$ for $V_{0}=0$ and $\Delta=0$. The momentum distribution was evaluated for various values of the modulation amplitude $\gamma=V_{0} /(\hbar \omega)$ and $\varphi_{m}=m \pi / 2$ at time $\mathbf{a} t=0$ and $\mathbf{b} t=T / 8$. The distribution exhibits additional momentum components separated by $\delta k=\pi /(2 a)$ whose amplitudes oscillate with period $T / 4, T=2 \pi / \omega$.

\subsubsection{Micro-motion Wannier-Stark ladder}

The Wannier-Stark ladder denotes a periodic potential with linearly increasing on-site potential along the direction of the lattice structure $\Delta_{m}=m \Delta$ as illustrated in Fig. 3.1b. For this lattice potential the micro-motion operator is determined according to

$$
\hat{\mathcal{M}}_{W S}(t)=\sum_{m}\left(-\frac{V_{0}}{\hbar \omega} \cos \left(\omega t+\varphi_{m}\right)+\frac{\Delta t}{\hbar} m\right) \hat{n}_{m}
$$

which is very similar to the case of the staggered superlattice potential. The modulationinduced component $\hat{\mathcal{M}}(t)_{\text {mod }}$ is again the same causing an equivalent micro-motion in quasimomentum space as illustrated in Fig. 3.2. The second component however differs from the two-site superlattice potential and is determined by

$$
\hat{\mathcal{M}}(t)_{\text {pot }}=\sum_{m}\left(\frac{\Delta t}{\hbar} m\right) \hat{n}_{m}
$$

For a driving frequency $\omega=\Delta / \hbar$ the micro-motion is associated with a constant drift in momentum space with $\delta k(t)=\omega t / a$. Hence, if the system is initially prepared in the ground state of the unperturbed lattice with $V_{0}=0$ and $\Delta=0$, the momentum distribution travels across the Brillouin zone while the corresponding amplitudes remain unchanged. In Figure 3.3b we illustrate the evolution for a state $|\psi(t)\rangle$ using Eqs. (3.69) and (3.71), where $|G S\rangle$ is again the ground state in the unperturbed lattice. After each period of the driving $T$ the momentum components travelled across the Brillouin zone once. This contribution to the micro-motion operator is independent of the on-site modulation. Consequently, all driving schemes based on the Wanner-Stark ladder will exhibit 

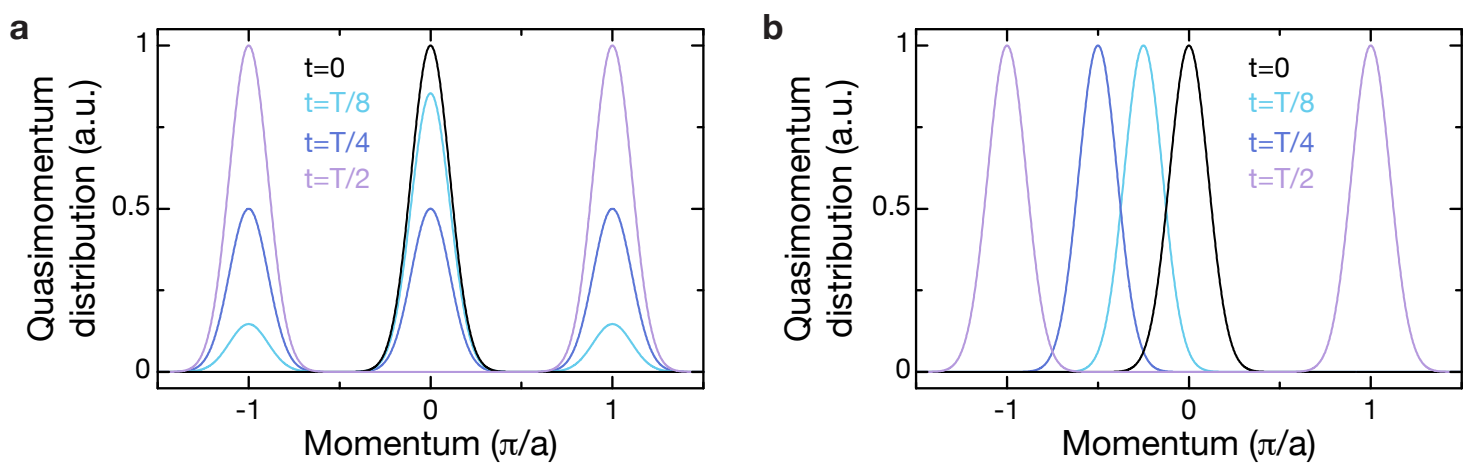

Figure 3.3: Typical momentum distribution after acting with the on-site-potential induced micro-motion operator $\hat{\mathcal{M}}(t)_{\text {pot }}$ on an initial state with zero quasimomentum. The initial state is the ground state of the unperturbed lattice potential with lattice constant $a$ for $V_{0}=0$ and $\Delta=0$. a The staggered on-site potential illustrated in Fig. 3.1a $(\Delta \neq 0)$ causes oscillations between the quasimomentum components at zero and $\pm \pi / a$ with period $T=h / \Delta$. $\mathbf{b}$ In the case of the Wannier-Stark ladder shown in Fig. 3.1b $(\Delta \neq 0)$ the micro-motion results in a constant drift of the quasimomentum components with $\delta k(t)=\Delta t / h \times 2 \pi / a$.

a drift of the momentum components in reciprocal space.

\subsection{Extension to two dimensions: artificial gauge fields}

In one dimension complex tunnel couplings can be engineered but they do not give rise to a magnetic flux. The schemes presented above can be further extended to two dimensions by introducing another lattice potential in the perpendicular direction. For the $2 \mathrm{D}$ lattice the tight-binding Hamiltonian can be written as

$$
\begin{aligned}
\hat{H}(t)= & \sum_{m, n}\left(-J_{x} \hat{a}_{m+1, n}^{\dagger} \hat{a}_{m, n}-J_{y} \hat{a}_{m, n+1}^{\dagger} \hat{a}_{m, n}+\text { h.c. }\right) \\
& +\sum_{m, n}\left(V_{0} \sin \left(\omega t+\varphi_{m, n}\right)+\Delta_{m}\right) \hat{n}_{m, n},
\end{aligned}
$$

where $J_{y}$ is the tunneling amplitude along $y$. Note that tunneling is only inhibited along the $x$-direction with the static potential $\Delta_{m}$. The modulation contains site-dependent phases $\varphi_{m, n}$ which depend both on the $x$ - and $y$-coordinate and the position in the lattice is defined as $\mathbf{R}=m a \hat{\mathbf{e}}_{x}+n a \hat{\mathbf{e}}_{y}$, with $\hat{\mathbf{e}}_{y}$ the unit vector along $y$. The derivation of the effective Hamiltonian discussed above also applies to modulated 2D lattices. We perform the following unitary transformation as defined in Eqs. (3.37)-(3.38) using

$$
\hat{R}(t)=\exp \left[i \sum_{m, n}\left(-\frac{V_{0}}{\hbar \omega} \cos \left(\omega t+\varphi_{m, n}\right)+\frac{\Delta_{m} t}{\hbar}\right) \hat{n}_{m, n}\right]=\exp \left[i \sum_{m, n} \chi_{m, n}(t) \hat{n}_{m, n}\right] .
$$



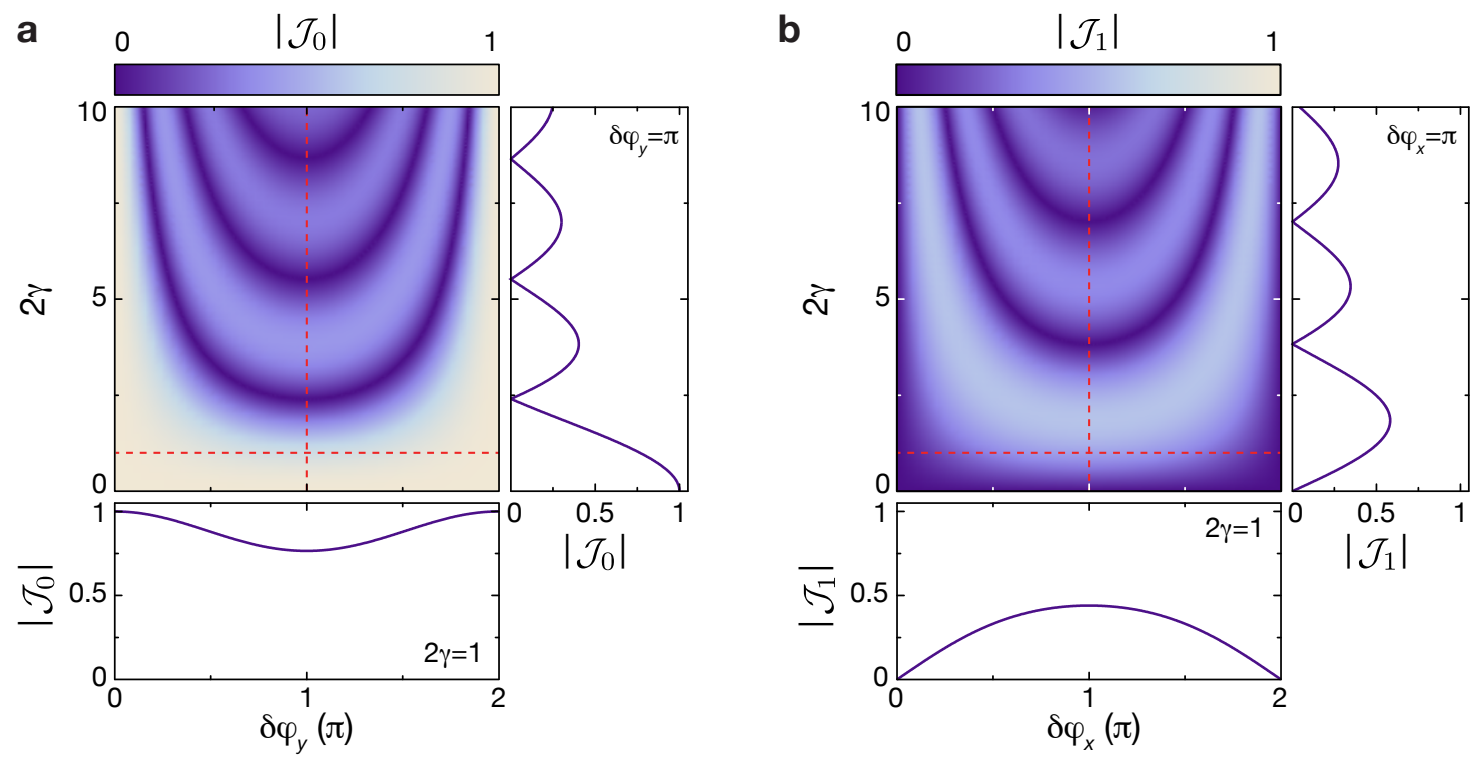

Figure 3.4: Bessel functions $\mathcal{J}_{0}\left(\eta_{0 y}\right)$ and $\mathcal{J}_{1}\left(\eta_{0 x}\right)$ for the zero- and first-order process. a Tunneling along the $y$-direction is proportional to the zeroth order Bessel function of the first kind $J / J_{y}=\mathcal{J}_{0}\left(\eta_{0 y}\right)$ as given in Eq. (3.78), which depends on the driving amplitude $\gamma=V_{0} /(\hbar \omega)$ and the phase difference $\delta \varphi_{y}=\varphi_{m, n+1}-\varphi_{m, n}$ of the on-site modulation. $\mathbf{b}$ Restored effective coupling along the $x$ axis, which is proportional to the first order Bessel function of the first kind $K / J_{x}=\mathcal{J}_{1}\left(\eta_{0 x}\right)$. It also depends on the driving amplitude $\gamma$ as well as the phase difference of the modulation along the corresponding axis $\delta \varphi_{x}=\varphi_{m+1, n}-\varphi_{m, n}$. The red dashed lines mark the values used for the calculations depicted in the side panels.

The transformed Hamiltonian can be written in the following form, where we have to take into account that the periodic modulation potentially affects the tunneling in both directions

$$
\begin{aligned}
\hat{\mathcal{H}}(t) & =-J_{x} \sum_{m, n}\left(\mathrm{e}^{i \eta_{m, n}^{x}(t)} \hat{a}_{m+1, n}^{\dagger} \hat{a}_{m, n}+\text { h.c. }\right) \\
& =-J_{y} \sum_{m, n}\left(\mathrm{e}^{i \eta_{m, n}^{y}(t)} \hat{a}_{m, n+1}^{\dagger} \hat{a}_{m, n}+\text { h.c. }\right),
\end{aligned}
$$

with the differential modulation amplitudes $\eta_{m, n}^{x}(t)=\chi_{m+1, n}(t)-\chi_{m, n}(t)$ and $\eta_{m, n}^{y}(t)=$ $\chi_{m, n+1}(t)-\chi_{m, n}(t)$ given by

$$
\begin{aligned}
& \eta_{m, n}^{x}(t)=-\eta_{0 x} \sin \left(\omega t+\frac{\varphi_{m+1, n}+\varphi_{m, n}}{2}\right)+\left(\Delta_{m+1}-\Delta_{m}\right) t / \hbar \\
& \eta_{m, n}^{y}(t)=-\eta_{0 y} \sin \left(\omega t+\frac{\varphi_{m, n+1}+\varphi_{m, n}}{2}\right)
\end{aligned}
$$

where 


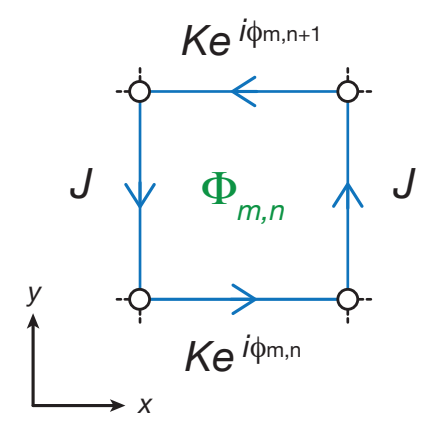

Figure 3.5: Magnetic flux per plaquette generated by Hamiltonian (3.79). The artificial flux is determined by the phase difference $\Phi_{m, n}=\phi_{m, n}-\phi_{m, n+1}$ using the convention that the magnetic field is pointing in the $+\hat{\mathbf{e}}_{z}$-direction. Its value only depends on the $y$-coordinate of the phase perpendicular to the direction of laser-assisted tunneling.

$$
\begin{aligned}
& \eta_{0 x}=\frac{2 V_{0}}{\hbar \omega} \sin \left(\frac{\varphi_{m+1, n}-\varphi_{m, n}}{2}\right)=: 2 \gamma \sin \left(\delta \varphi_{x} / 2\right), \\
& \eta_{0 y}=\frac{2 V_{0}}{\hbar \omega} \sin \left(\frac{\varphi_{m, n+1}-\varphi_{m, n}}{2}\right)=: 2 \gamma \sin \left(\delta \varphi_{y} / 2\right) .
\end{aligned}
$$

In the following we consider only on-site potentials with $\left|\Delta_{m+1}-\Delta_{m}\right|=\Delta$. Hence, for resonant laser-assisted tunneling $\hbar \omega=\Delta$, the effective time-independent Hamiltonian can be written as

$$
\hat{\mathcal{H}}_{F}=-\underbrace{J_{x} \mathcal{J}_{1}\left(\eta_{0 x}\right)}_{=: K} \sum_{m, n} \mathrm{e}^{i \phi_{m, n}} \hat{a}_{m+1, n}^{\dagger} \hat{a}_{m, n}-\underbrace{J_{y} \mathcal{J}_{0}\left(\eta_{0 y}\right)}_{=: J} \sum_{m, n} \hat{a}_{m, n+1}^{\dagger} \hat{a}_{m, n}+\text { h.c., }
$$

where the phases $\phi_{m, n} \propto\left(\varphi_{m+1, n}+\varphi_{m, n}\right) / 2$ depend on the phase distribution $\varphi_{m, n}$ as well as on the particular shape of the potential $\Delta_{m}$ (Sect. 3.2). Along the $x$-direction tunneling is restored by the periodic modulation resulting in an effective tunnel coupling $K$ accompanied by a complex phase $\phi_{m, n}$. In the perpendicular direction tunneling is potentially renormalized but real. In the limit $V_{0} \ll \hbar \omega$ the effective coupling $J$ is determined by

$$
\frac{J}{J_{y}}=1-\frac{1}{4} \eta_{0 y}^{2}+\mathcal{O}\left(\eta_{0 y}^{4}\right) \simeq 1-\gamma^{2} \sin ^{2}\left(\delta \varphi_{y} / 2\right)
$$

For small driving amplitudes the coupling is essentially given by the bare coupling $J_{y}$ and it decreases with increasing modulation strength $\gamma$. Additionally it also depends on the phase difference between neighboring sites $\delta \varphi_{y}$ (Fig. 3.4a). If neighboring sites are modulated in phase the differential modulation amplitude vanishes and the bare tunneling remains unchanged. The largest effect occurs for $\delta \varphi_{y}=\pi$ where the differential 
modulation amplitude is largest. A similar situation occurs along the $x$-direction with the difference that without the driving, tunneling is inhibited by the potential energy offset $\Delta$. Its strength $K$ depends on the phase difference $\delta \varphi_{x}$ and increases linearly with the driving amplitude $\gamma$. In the limit $V_{0} \ll \hbar \omega$ it is given by

$$
\frac{K}{J_{x}}=\frac{1}{2} \eta_{0 x}+\mathcal{O}\left(\eta_{0 x}^{3}\right) \simeq \gamma \sin \left(\delta \varphi_{x} / 2\right) .
$$

If the modulation is in phase $\left(\delta \varphi_{x}=0\right)$ between neighboring sites tunneling cannot be restored and the largest effect occurs once more for $\delta \varphi_{x}=\pi$. This phase dependence allows for a local control of the laser-assisted tunneling as discussed in chapter 7 and 8 $[66,73]$.

The Peierls phase-factors $\phi_{m, n}$ in the effective Hamiltonian (3.79) appear along the direction of the laser-assisted tunneling and the flux per plaquette is defined according to

$$
\Phi_{m, n}=\phi_{m, n}-\phi_{m, n+1},
$$

where the direction of the magnetic field is defined along the $+\hat{\mathbf{e}}_{z}$-direction. A schematic drawing of the relevant tunnel couplings is shown in Fig. 3.5. Note that the value of the flux only depends on the phase of the modulation along the $y$-axis $\delta \varphi_{y}$. The evolution of the phase along the $x$-axis $\delta \varphi_{x}$ only determines the strength of the effective coupling. The model system presented in this chapter naturally gives rise to artificial fluxes that are on the order of $2 \pi$ and therefore constitute good candidates to enter the regime of very strong magnetic fields on the order of one magnetic flux quantum. Similar methods were developed for photonic crystals [82] and ion traps [65, 132]. 


\section{Chapter 4}

\section{Overview of the experimental setup and measurement techniques}

The experiments presented in the context of this thesis were performed with bosonic atoms $\left({ }^{87} \mathrm{Rb}\right)$. For all measurements the atoms were first cooled below the critical temperature for Bose-Einstein condensation (BEC) [133, 134], which for our experimental parameters corresponds to about $100 \mathrm{nK}$. After that the atoms were loaded into optical lattice potentials where the final measurements were carried out. The main experimental apparatus was designed about 15 years ago and is well described in Refs. [135, 136] and number of successive PhD theses [137-140]. During the past several modifications have been made in particular regarding the final stage of the BEC preparation, which takes place in a crossed optical dipole trap. The most recent description of the current experimental setup can be found in Ref. [141].

The main apparatus consists of two vacuum chambers connected via a differential pumping stage. This allows for a pressure gradient between the two chambers, such that the pressure in the science chamber, where the experiments take place, can be a few orders of magnitude smaller than the one in the chamber to which the Rubidium reservoir is attached. In this first chamber the pressure $p \approx 10^{-8}-10^{-9}$ mbar is dominated by the partial pressure of Rubidium, which allows for an efficient loading into a standard three-dimensional magneto-optical trap (MOT) [142]. After pre-cooling the atoms in this configuration they are transported to an ultra-high vacuum (UHV) or science chamber, with $p \approx 10^{-11}$ mbar, using a magnetic transportation scheme [135, 143]. There, the atoms are cooled further to reach quantum degeneracy via evaporative cooling (Sect. 4.1). Subsequently they are loaded into optical lattice and superlattice potentials (Sect. 4.2-4.3). Relevant experimental techniques for state manipulation and detection are introduced at the end of this chapter (Sect. 4.4-4.6). 


\subsection{Towards quantum degeneracy}

The cooling sequence is carried out in three distinct sections of the main apparatus (Fig. 4.1) and involves several stages: pre-cooling in a magneto-optical trap (red coils) and optical molasses, magnetic transfer to the science chamber (green coils) and a twostage evaporative cooling in a magnetic quadrupole (yellow coils) and a crossed optical dipole trap.

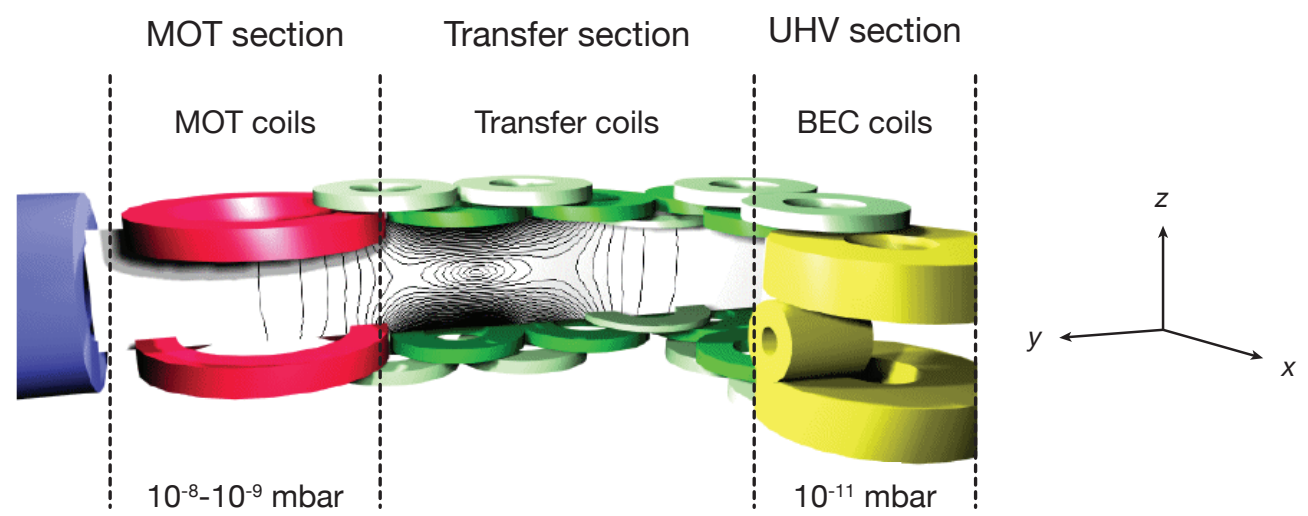

Figure 4.1: Schematic drawing of the coil arrangement around the vacuum chamber. The experimental apparatus is divided into three parts: the first chamber with a magneto-optical trap (MOT, red coils) for initial trapping and cooling of the atoms, the transfer section (magnetic transport using the green coils) with the differential pumping stage and the ultra-high vacuum (UHV) or science chamber with a magnetic quadrupole trap (yellow coils), where the atoms are further cooled evaporatively. The black lines show the equipotential lines of the trapping potential at a certain time during the magnetic transfer. (Figure adapted from Ref. [136])

Magneto-optical trap and optical molasses Each experimental cycle starts by trapping ${ }^{87} \mathrm{Rb}$ atoms in a standard three-dimensional magneto-optical trap (3D-MOT) [142, $144,145]$. Cooling in such a trap is achieved via the combined effect of slowing down the atoms due to absorption of photons and the spacial confinement of the underlying magnetic trap (Fig. 4.1). In a 3D-MOT the atoms are typically cooled along all three spatial directions using two counter-propagating laser beams along each direction. The MOT is operated on the $D_{2}$-line of Rubidium $\left(\lambda_{D 2}=780 \mathrm{~nm}\right)^{1}$, which corresponds to the $\left|S_{1 / 2}\right\rangle \rightarrow\left|P_{3 / 2}\right\rangle$ transition as illustrated in Fig. 4.2a [146]. After $15 \mathrm{~s}$ operation time about $10^{9}$ atoms are obtained with final temperatures below $1 \mathrm{mK}$. In order to reach higher densities the magnetic field gradients are increased at the end of the MOT phase [147]; this causes additional heating which is subsequently compensated by cooling the atoms in an optical molasses [148]. At the end final temperatures in the low $\mu \mathrm{K}$-regime

\footnotetext{
${ }^{1}$ TOPTICA, DL-PRO 100 (output power: $80 \mathrm{~mW}$ ); TOPTICA, DL-PRO 100 with an additional tapered amplifier (output power: $1 \mathrm{~W}$ )
} 
a

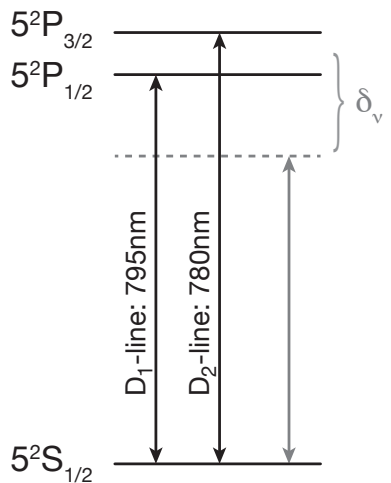

b

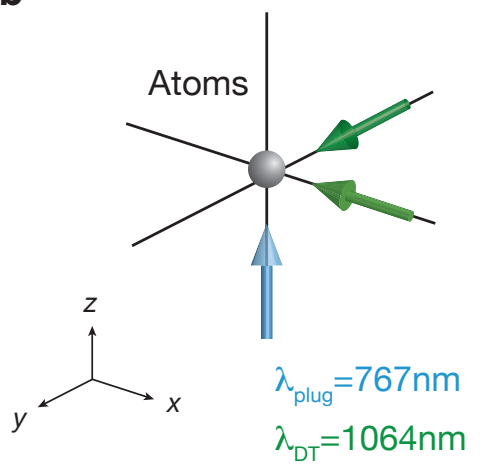

C

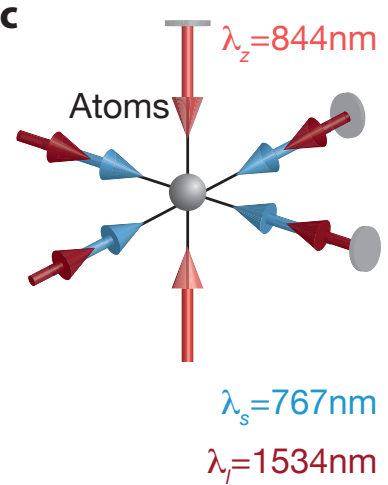

Figure 4.2: Illustration of the atomic transitions and laser beams used in the experimental setup. a Simplified level scheme of ${ }^{87} \mathrm{Rb}$ with $\left|S_{1 / 2}\right\rangle$ being the ground state and $\delta_{v}$ the detuning relative to the atomic transition $|S\rangle \rightarrow|P\rangle$. Laser beams with negative frequency detuning $\delta_{v}<0$ are called red-detuned, while for positive detuning $\delta_{v}>0$ they are denoted as blue-detuned. b Schematics of the laser-beam configuration involved in the two-stage evaporative cooling (see main text). The optical plug $\left(\lambda_{\text {plug }}=767 \mathrm{~nm}\right)$ used to minimize Majorana losses during the rfevaporation is aligned along the vertical direction. The second evaporation step is performed in a crossed optical dipole trap $\left(\lambda_{\mathrm{DT}}=1064 \mathrm{~nm}\right)$, where the beams are aligned along the principal axes in the horizontal plane. c Schematic drawing of the optical lattices, which are created by retro-reflected laser beams. The corresponding mirrors are illustrated as gray plates. The vertical lattice is red-detuned to the atomic transition with $\lambda_{z}=844 \mathrm{~nm}$. In the horizontal plane there are two lattice potentials along each axis, a long and a short one with wavelengths $\lambda_{s}=767 \mathrm{~nm}$ and $\lambda_{l}=1534 \mathrm{~nm}$ respectively. The superposition of these two standing waves generates a bichromatic superlattice potential. The atomic cloud is illustrated as a gray sphere.

are reached. As a last step, all atoms are optically pumped into to the $\left|F=1, m_{F}=-1\right\rangle$ state of the ground-state manifold $\left|S_{1 / 2}\right\rangle$, which is a low field seeking state. To initialize the magnetic transport the atoms are loaded into a magnetic quadrupole trap with efficiencies up to $70 \%$. The lifetime in this trap is about $\tau \simeq 8 \mathrm{~s}$ [141], where $\tau$ is defined as the $1 / e$-decay of the atom number.

Magnetic transport Starting from the MOT-chamber the atoms are transferred magnetically across an "L-shaped" distance of about $33 \mathrm{~cm}$ to the science chamber; $20 \mathrm{~cm}$ along the $y$-axis and $13 \mathrm{~cm}$ along $x$. The efficiency of the transfer can be up to $98 \%$ and takes $4 \mathrm{~s}$ in the current setup. The coil configuration consists of nine pairs of overlapping quadrupole coils (Fig. 4.1) that are designed such that the trap geometry stays constant during the transport to minimize heating effects [143]. At the end of the transport the atoms are loaded into a final magnetic quadrupole trap in the science chamber (large yellow coils in Fig. 4.1). There are two main advantages arising from the transport design. First, it allows for a simple implementation of the MOT because it is efficiently loaded from the background vapor at a relatively high pressure; there is no need for an addi- 
tional deceleration of the atoms by using a Zeeman slower [149] or a two-dimensional MOT [150]. At the same time the pressure in the science chamber can be much lower which increases the lifetime of the atoms by roughly one order of magnitude [141]. The second advantage is the large optical access around the science chamber due to the spatial separation of the MOT setup. This increases the experimental flexibility regarding the implementation of additional laser beams for state preparation and manipulation.

Evaporative cooling The final cooling stage consists of a two-stage evaporative cooling process [151-153]: Radio-frequency (rf) induced evaporation in an optically plugged magnetic quadrupole trap and forced evaporation in a crossed optical dipole trap. The general operation principle of evaporative cooling is to successively remove the hottest atoms from the trap and letting the remaining atoms rethermalize while increasing the phase-space density at the same time. Evaporative cooling with Alkali atoms was first demonstrated in magnetic traps [154, 155]. Thereby rf-fields are used to transfer atoms from a magnetically trappable to a magnetically untrappable state in an energy selective manner. The trap configuration is kept constant during the evaporation, which allows for an efficient rethermalization of the atoms by maintaining large collision rates.

During the rf-evaporation in the magnetic quadrupole trap atoms can undergo nonadiabatic spin flips (Majorana losses) at the center of the trap where the magnetic field vanishes. This potentially leads to undesired atom losses. To overcome this problem various other types of traps could be used which do not exhibit a zero-crossing of the magnetic field, such as TOP (time-averaged orbiting potential) [154], Ioffe [156] or Ioffetype traps [157]. In previous experiments the small yellow coil in Fig. 4.1 was used to realize a Ioffe-type trap. In the current experimental setup we use a tightly focused blue-detuned laser beam ${ }^{2}\left(\lambda_{\text {plug }}=767 \mathrm{~nm}, \delta_{v}>0\right.$ ) along the $z$-direction (Fig. $4.2 \mathrm{~b}$ ), which is focused at the atom position to a waist of about $20 \mu \mathrm{m}$ with a typical power of $\sim 500 \mathrm{~mW}$. It creates a repulsive potential [158] at the point of zero-magnetic field and prevents the atoms from reaching field regimes where the probability for Majorana losses is high. The total rf-evaporation takes $8.5 \mathrm{~s}$ and the final cut-off frequency is $v_{\mathrm{rf}}=2 \mathrm{MHz}$. After this pre-evaporation stage the atoms are loaded into a crossed optical dipole trap ${ }^{3}$ with wavelength $\lambda_{D T}=1064 \mathrm{~nm}$ (Fig. 4.2b) and a maximum potential depth of about $U_{0}=k_{B} \times 40 \mu \mathrm{K}$, where $k_{B}$ is the Boltzmann constant.

In principle evaporation in an optical trap can be achieved by lowering the intensities of the optical dipole trap beams, however, this leads to a decrease of the collision rate, which may in turn cause inefficient rethermalization [159]. One possibility to overcome this issue and to reach runaway evaporative cooling is to apply an external force during the evaporation [160]. This causes only a weak deconfinement of the dipole trap and

\footnotetext{
${ }^{2}$ Chapter 5: Ti:sapphire laser Coherent, MBR-110 (output power: $4.5 \mathrm{~W}$ ) pumped by Coherent Verdi V18 (output power: $18 \mathrm{~W}$ ); chapter 6-8: Ti:sapphire laser M Squared, SolsTis (output power: 2.4 W) pumped by Lighthouse Photonics Sprout-G V10 (output power: $10 \mathrm{~W}$ )

${ }^{3}$ YLR-20-LP IPG-Laser, output power: 20W
} 
forces the hottest atoms to leave the trap in the direction of the force. In our experiments we use gravity as a force, which allows us to reach quantum degeneracy within an additional evaporation time of $8 \mathrm{~s}$.

\subsection{Optical lattice potentials}

All measurements presented in the scope of this thesis were carried out in periodic optical potentials [158] of different dimensionality (1D-3D) by adiabatic loading of a BEC into several standing waves of laser light [161]. Each standing-wave potential is created by focusing a far-detuned laser beam onto the atom position with a waist of typically $\sim 125 \mu \mathrm{m}$ and retro-reflecting it with a mirror (Fig. 4.2c). Depending on the sign of the frequency detuning $\delta_{v}$ relative to the atomic transition (Fig. 4.2a) the atoms are either located at the intensity maxima (red detuned, $\delta_{v}<0$ ) or minima (blue detuned, $\delta_{v}>0$ ). These periodic structures are called optical lattice potentials. In the horizontal plane there are two blue detuned lattices with wavelength $\lambda_{s}=767 \mathrm{~nm}$ for which we use the same laser as for the optical plug described in the previous section. The vertical lattice ${ }^{4}$ is red detuned with wavelength $\lambda_{z}=844 \mathrm{~nm}$ (Fig. 4.2c). Each of these beams passes through an acousto-optic modulator which is employed to stabilize the intensity of the beams individually with a feedback loop [162] and to introduce a frequency offset of at least $20 \mathrm{MHz}$ between lattice beams, that are generated with the same laser, in order to avoid cross-interference. Additionally the polarizations of the beams are chosen to be orthogonal to further reduce possible cross-interference terms. Neglecting the overall confinement due to the Gaussian beam profile the 3D potential can be described by the following equation

$$
V_{\text {Lat }}(\mathbf{r})=-V_{x} \cos ^{2}\left(k_{s} x\right)-V_{y} \cos ^{2}\left(k_{s} y\right)-V_{z} \cos ^{2}\left(k_{z} z\right),
$$

where $V_{i}, i=\{x, y, z\}$ is the potential depth and $k_{i}=2 \pi / \lambda_{i}, i=\{s, z\}$ is the wave vector. The typical energy scale of an optical lattice is determined by the corresponding photon recoil energy $\mathrm{E}_{\mathrm{ri}}=\hbar^{2} k_{i}^{2} /(2 m), i=\{s, z\}$, where $m$ is the mass of the atom. The motion of the atoms in such a periodic crystal-like structure mimics the behavior of electrons in a solid state crystal, where the atoms play the role of the electrons $[29,44]$. The typical lattice constant $a_{i}=\lambda_{i} / 2$ of optical crystals is several orders of magnitude larger than typical lattice spacings in a real solid, which are on the order of a few angstroms. This opens up many new possibilities to study condensed matter Hamiltonians by looking at observables not accessible in typical solid state experiments as demonstrated e.g. with high-resolution quantum gas microscopes that can resolve single atoms on individual lattice sites [49, 50, 163].

The Schrödinger equation of a particle in a periodic potential can be solved using Bloch's

\footnotetext{
${ }^{4}$ Ti:sapphire laser Coherent, MBR-110 (output power: $1.5 \mathrm{~W}$ ) pumped by Coherent Verdi V10 (output power: $10 \mathrm{~W}$ )
} 


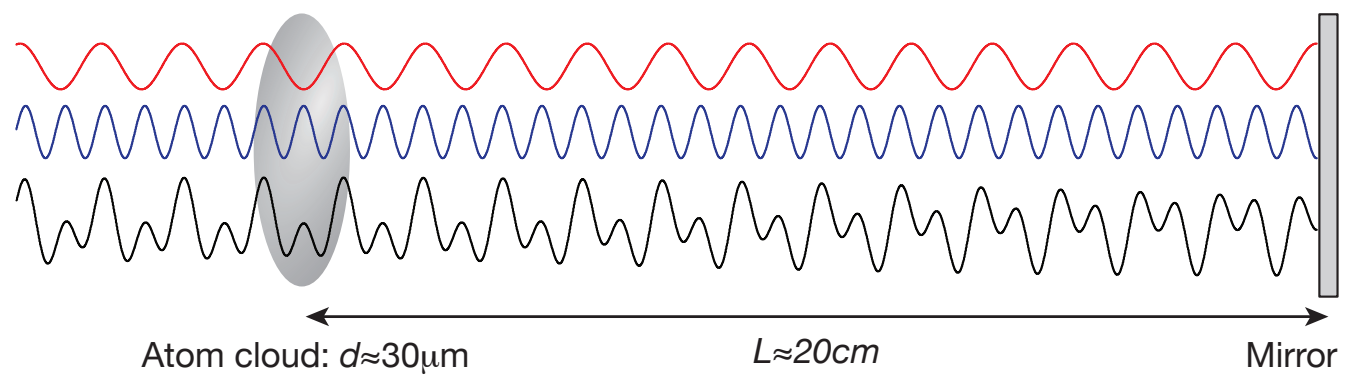

Figure 4.3: Illustration of the experimental setup of the bichromatic superlattice. The superlattice potential (black) consists of two standing waves with $\lambda_{s}=767 \mathrm{~nm}$ (blue) and $\lambda_{l}=2 \lambda_{s}$ (red). Due to a small frequency offset $\Delta v$ between the two lasers the standing waves accumulate a relative phase until they reach the atom position which is proportional to the distance $L$ between the atoms and the mirror. The relative phase is approximately constant over the extent of the cloud as $d \ll L$. Note that distances are not to scale.

theorem [84, 85], which is very similar to the more general Floquet's theorem, that was introduced in Sect. 3.1 for time-periodic Hamiltonians. For shallow lattices $V<5 E_{r}$ the atoms are delocalized over several lattice sites and the system is well described in terms of Bloch functions. For deeper lattice potentials the atomic wave functions become more and more localized and it is convenient to choose a localized basis to describe the dynamics. These are the so-called Wannier functions, which are a superposition of Bloch functions. Considering only the lowest vibrational band the dynamics can be described by the Bose-Hubbard Hamiltonian [43]

$$
\hat{H}_{\mathrm{BH}}=-J \sum_{\langle m, n\rangle} \hat{a}_{m}^{\dagger} \hat{a}_{n}+\sum_{m} \Delta_{m} \hat{n}_{m}+\frac{1}{2} U \sum_{m} \hat{n}_{m}\left(\hat{n}_{m}-1\right),
$$

where $\hat{a}_{m}^{\dagger}$ and $\hat{a}_{m}$ are the bosonic creation and annihilation operators on site $m$ and $\hat{n}_{m}$ is the corresponding number operator. The Hubbard parameter $J$ is the tunneling matrix element between neighboring sites $n$ and $m, \Delta_{m}$ is the potential energy offset on the corresponding site and $U$ determines the on-site interaction of two atoms that are located on the same lattice site. For the measurements described in the context of this thesis the on-site interaction $U$ is neglected, unless stated otherwise, because it is either much smaller than all other energy scales of the system or the experiments were performed with single particles in isolated few-site potentials.

\subsection{Superlattice potential}

Besides the dimensionality also the unit cell of the crystal can be modified. By implementing superlattice potentials in the horizontal plane a periodic potential whose unit cell contains two or four non-equivalent lattice sites can be realized. Along each of the 
two axes a bichromatic superlattice potential can be created by superimposing an additional standing wave ${ }^{5}$ with wavelength $\lambda_{l}=2 \lambda_{s}=1534 \mathrm{~nm}$ (Fig. 4.2c). The phase of each standing wave is fixed at the position of the retro-reflecting mirror, however, by introducing a small frequency difference $\Delta v=2 v_{l}-v_{s}, v_{l(s)}=c / \lambda_{l(s)}$, between the two lasers the two standing waves accumulate a relative phase until they reach the atom position according to

$$
\varphi_{S L}=\frac{2 \pi}{c} \Delta v L,
$$

where $c$ is the speed of light and $L$ is the distance between the retro-reflecting mirror and the position of the atomic cloud (Fig. 4.3). The variation of the phase over the extent of the atomic cloud with a typical diameter of $d \approx 30 \mu \mathrm{m}$ can be safely neglected. For our experimental parameters it varies by less than $1 \mathrm{mrad}$. A detailed description of the setup and the frequency stabilization can be found in Ref. [139, 141, 164]. The resulting superlattice potential along $x$ can be written in the following form

$$
V(x)=V_{x l} \cos ^{2}\left(k_{l} x+\varphi_{S L}^{x} / 2\right)+V_{x} \cos ^{2}\left(k_{s} x\right),
$$

where $k_{l}=2 \pi / \lambda_{l}=k_{s} / 2$ and $V_{x l}$ is the depth of the long lattice. The lattice depths $V_{x}$ and $V_{x l}$ can be controlled independently. The superlattice potential along $y$ is given by an analog expression, where $V_{y l}$ and $V_{y}$ are the corresponding lattice depths along $y$. The relative phases $\varphi_{S L}^{x}$ and $\varphi_{S L}^{y}$ can be adjusted individually for the two axes and determine the shape of the superlattice potential as illustrated in Fig. 4.4a.

\subsubsection{Phase calibration}

To calibrate the phase of the superlattice potential [139] we typically load a BEC adiabatically within $200 \mathrm{~ms}$ into the ground state of a three-dimensional optical lattice using the following parameters: $V_{x}=V_{y}=5.0(2) \mathrm{E}_{\mathrm{rs}}, V_{z}=5.0(2) \mathrm{E}_{\mathrm{z}}$ and $V_{x l}=10.0(3) \mathrm{E}_{\mathrm{rl}}$, with $\mathrm{E}_{\mathrm{rl}}=\hbar^{2} k_{l}^{2} /(2 m)$; the phase $\varphi_{S L}^{x}$ is adjusted for each measurement by controlling the frequency difference $\Delta v_{x}$ as defined in Eq. (4.3). The lattices are shallow enough to remain in the superfluid regime [44] where the atoms are delocalized over the lattice and the phase of the ground-state wave function between different lattice sites is constant. After releasing the atoms suddenly from the trap the corresponding momentum distribution can be obtained after TOF [29] using standard absorption imaging [165]. For the symmetric double well configuration $\left(\varphi_{S L}^{x}=0\right)$ the periodicity of the superfluid wave function is determined by the lattice constant of the short lattice $a=\lambda_{s} / 2$. For this reason we observe a distribution with momentum peaks separated by $k_{x}= \pm 2 k_{s}$ (Fig. 4.4b), which is the reciprocal lattice vector of the periodic potential. The envelope

\footnotetext{
${ }^{5}$ Chapter 5-7: Two Erbium doped fiber amplifier from NP Photonics (output power: $5 \mathrm{~W}$ ) one for each axis; chapter 8: Laser for $x$-lattice was changed to: Seed laser RIO Orion Laser Source (output power: $5-10 \mathrm{~mW}$ ) with fiber amplifier Nufern NuAMP (output power: $6 \mathrm{~W}$ )
} 
a

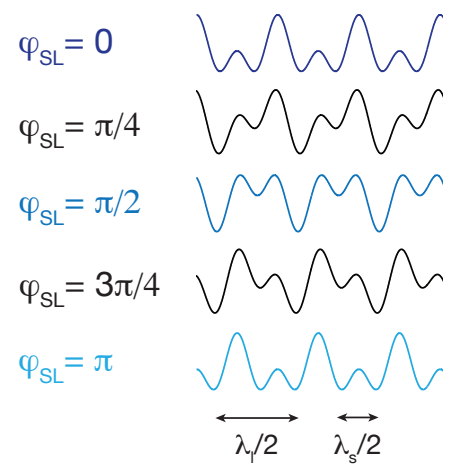

b

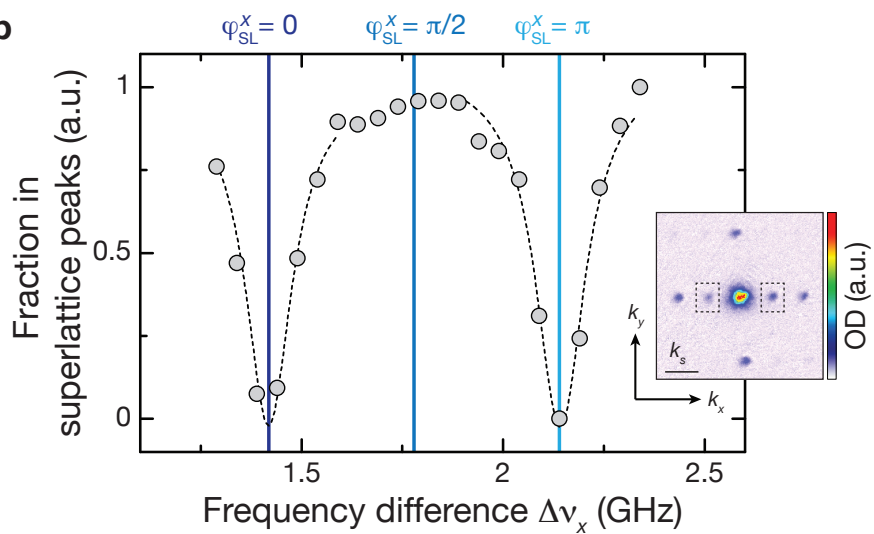

Figure 4.4: Influence of the relative phase between the two standing waves used to create the superlattice potential. a Illustration of the superlattice potential given in Eq. (4.4) for $V_{l}=V$ and different values of the relative phase $\varphi_{S L}$. For $\varphi_{S L}=\{0, \pi, \ldots\}$ we refer to the potential as symmetric double-well configuration while for $\varphi_{S L}=\{\pi / 2,3 \pi / 2, \ldots\}$ we denote it as staggered superlattice. Any value in between results in a tilted double-well configuration. $\mathbf{b}$ Phase calibration of the superlattice potential along $x$. Fraction of atoms in the momentum components $k_{l}= \pm 2 k_{l}= \pm k_{s}$ (dashed boxes in the inset) corresponding exclusively to the long lattice spacing $a_{l}=\lambda_{l} / 2$ as a function of the superlattice phase $\varphi_{S L}^{x}$, which is controlled by the frequency difference $\Delta v_{x}$ according to Eq. (4.3). The color code illustrates the connection between the shape of the superlattice potential and the measured fraction. For the symmetric double-well configuration the lattice constant is given by the short lattice spacing $a_{s}=\lambda_{s} / 2$ and the amplitude of the momentum components at $k_{x}= \pm 2 k_{l}$ almost disappears. The separation between the two minima $\Delta v_{x}=0.74(1) \mathrm{GHz}$ corresponds to a phase shift $\delta \varphi_{S L}^{x}=\pi$. The dashed lines are guides to the eye. The inset shows a typical experimental image obtained after $10 \mathrm{~ms}$ TOF using standard absorption imaging.

of the pattern is determined by the Fourier transform of the on-site wave function.

If the phase is increased away from the symmetric configuration the double-well potential becomes more and more asymmetric and momentum components at $k_{x}= \pm 2 k_{l}$ appear in the TOF images. The amplitude of the momentum components depends on the relative population of the atoms on the two sites of the unit cell. Above a certain value of the phase atoms only populate the sites with lower energy and the periodicity is solely given by the long lattice constant $a_{l}=\lambda_{l} / 2$. At this value of the phase the fraction of atoms diffracted into the momentum peak at $k_{x}= \pm 2 k_{l}$ is largest. Increasing the phase even further does not influence the distribution anymore since only the lower energy sites are populated and the particular shape of the potential does not influence the periodicity of the ground-state wave function.

For even larger values of the phase again a symmetric double-well configuration is reached where the peaks at $k_{x}= \pm 2 k_{l}$ almost disappear. The fraction of atoms appearing at $k_{x}= \pm 2 k_{l}$ is expected to change periodically as a function of the superlattice phase in agreement with the experimental data depicted in Fig. 4.4. This calibration 

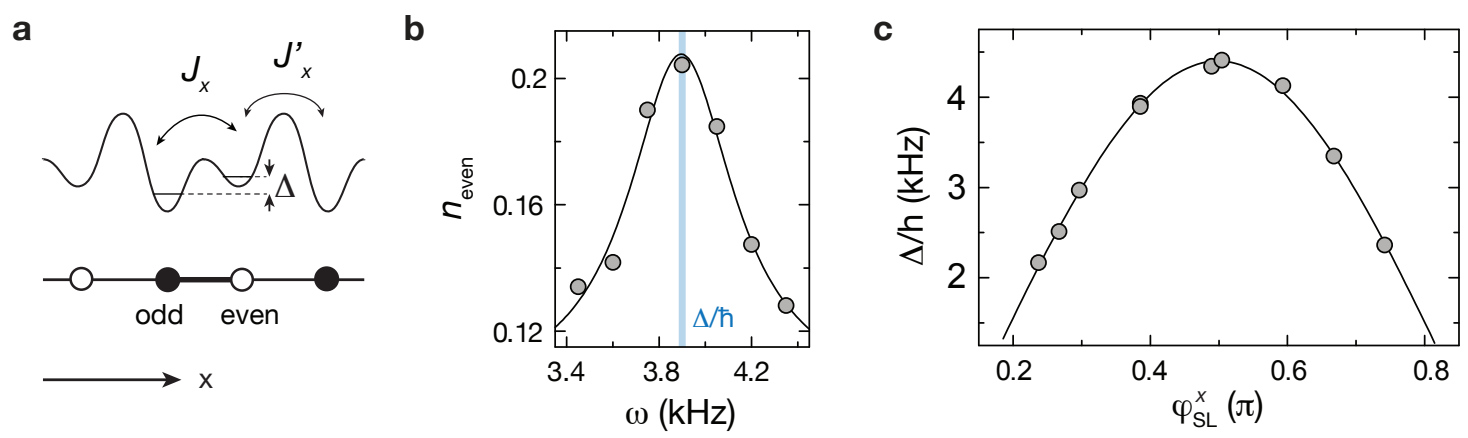

Figure 4.5: Calibration of the superlattice phase $\varphi_{S L}^{x}$ using spectroscopy measurements. a Schematic drawing of the superlattice potential with energy offset $\Delta$, inner-well coupling $J_{x}$ and inter-well coupling $J_{x}^{\prime}$. b Typical experimental data obtained from a spectroscopy measurement for $\varphi_{S L}^{x}=0.39(2) \pi$. The fraction of atoms transferred to even sites is measured as a function of the modulation frequency $\omega$. The solid line shows the fit of a Lorentzian to determine the resonance condition $\omega=\Delta / \hbar$. c Measured energy offset $\Delta$ obtained from spectroscopy measurements as shown in (a) as a function of the superlattice phase $\varphi_{S L}^{x}$. The offset exhibits a maximum for $\varphi_{S L}^{x}=\pi / 2$, which corresponds to the staggered superlattice potential. The solid line is a guide to the eye. (Figure adapted from Ref. [69])

method is very well suited for a first calibration of the superlattice phase [141, 164]. For a more precise characterization of the superlattice potential spectroscopy measurements can be performed as discussed in the following section.

\subsubsection{Calibration of the staggered superlattice potential}

Depending on the value of the superlattice phase $\varphi_{S L}$ an energy offset $\Delta$ is introduced between neighboring sites (Fig. 4.5a), which exhibits a maximum for $\varphi_{S L}=\pi / 2$ (Fig. 4.4a). This offset can be determined experimentally with spectroscopy measurements using lattice modulation or laser-assisted tunneling [69-71, 126], see also chapter 3. If the energy offset is much larger than the coupling between neighboring sites $\Delta \gg J, J^{\prime}$ tunneling is inhibited and all dynamics is frozen along the corresponding axis. By applying a periodic driving with frequency $\hbar \omega=\Delta$ tunneling can be restored resonantly. In this section calibration measurements of the superlattice potential along $x$ are presented (Fig. 4.5).

The experimental sequence started by loading the atoms adiabatically within $200 \mathrm{~ms}$ into a 3D optical potential. The lattice parameters were $V_{x l}=5.0(2) \mathrm{E}_{\mathrm{rl}}, V_{x}=9.0(3) \mathrm{E}_{\mathrm{rs}}$, $V_{y}=20(1) \mathrm{E}_{\mathrm{rs}}, V_{z}=20(1) \mathrm{E}_{\mathrm{z}}$ and $\varphi_{S L}^{x}>0$. The vertical lattice was used to isolate different planes but in general it has no influence on the calibration measurements. The ground state in this superlattice potential corresponds to a state, where all atoms occupy the lower energy sites, which are denoted as odd sites (Fig. 4.5a). The energy offset was chosen to be large $\Delta \gg J_{x}, J_{x}^{\prime}$ compared to the bare hopping amplitudes 
in order to inhibit tunneling to even sites. After suddenly switching on the modulation facilitated by the setup described in Sect. 5.3, the fraction of atoms transferred to even sites $n_{\text {even }}=N_{\text {even }} / N$ was measured as a function of the modulation frequency $\omega$ (Fig. 4.5b); here $N$ is the total atom number and $N_{\text {even }}$ the atom number on even sites, which was evaluated using the site-resolved detection technique introduced in Sect. 4.6. In all spectroscopy measurements the modulation was switched on for less than half a Rabi oscillation, $t<h /(4 K)$, where $K$ is the restored tunnel coupling given in Eq. (3.81) for $\delta \varphi_{x}=\pi / 2$. The measured transfer exhibits a maximum if the frequency difference is resonant with the energy offset $\omega=\Delta / \hbar$.

Note that there can be corrections to the resonance frequency on the order of $J_{x} / \Delta$. Related effects are discussed in Sect. 3.1.4 for the case of a two-level system. The resonance frequency which is calibrated experimentally using spectroscopy measurements always corresponds to the exact energy gap $E_{\text {gap }}$ between the energy levels as defined in Eq. (3.44). For typical experimental parameters $\Delta / J_{x} \simeq 30$ the resonance condition is well approximated by $\omega=\Delta / \hbar$ and corrections of order $J_{x} / \Delta$ can be safely neglected in the theoretical discussion. In Sect. 5.7, however, we are going to discuss an exception, where $\Delta / J_{x} \simeq 3.56$ and this approximation is not valid anymore. In this case the exact resonance condition has to be incorporated in the derivation of the effective Hamiltonian.

Beyond that the energy offset $\Delta$ was measured as a function of the superlattice phase $\varphi_{S L}^{x}$ as illustrated in Fig. 4.5c. It exhibits a maximum at $\varphi_{S L}^{x}=\pi / 2$, which corresponds to the staggered superlattice configuration. For our parameters the maximum energy offset was evaluated to be $\Delta / h=4.4(1) \mathrm{kHz}$. The effective coupling strengths for all measurements depicted in Fig. $4.5 \mathrm{~b}$ and c were on the order of $K / h=30 \mathrm{~Hz}$ [69]. The maximum amplitude of the resonance shown in Fig. $4.5 \mathrm{a}$ is $n_{\mathrm{even}}=0.5$, which is a state with equal population on all lattice sites.

\subsection{Calibration of the tunnel coupling}

The tunnel coupling between neighboring sites is determined experimentally by observing Josephson oscillations in isolated double well potentials, where the inner-well coupling $J$ is much larger than the inter double-well coupling $J^{\prime}$, so that all dynamics is restricted within the two sites of the double-well potential (Fig. 4.5a). The measurements discussed in the following section were performed along the $x$-axis making use of the laser-assisted tunneling method described in Sect. 5.3. We note however that this scheme is very general and can be applied equally well to measure bare tunnel couplings or renormalization of the tunneling in the presence of a periodic on-site modulation as derived in Eq. (3.80). Here we apply a periodic driving with amplitude $V_{0}$, frequency $\hbar \omega=\Delta$ and a phase difference $\delta \varphi_{x}=\pi / 2$ between neighboring sites, such that the restored resonant tunnel coupling is given by $K=J_{x} \mathcal{J}_{1}\left(\sqrt{2} V_{0} / \Delta\right)$ as determined by 

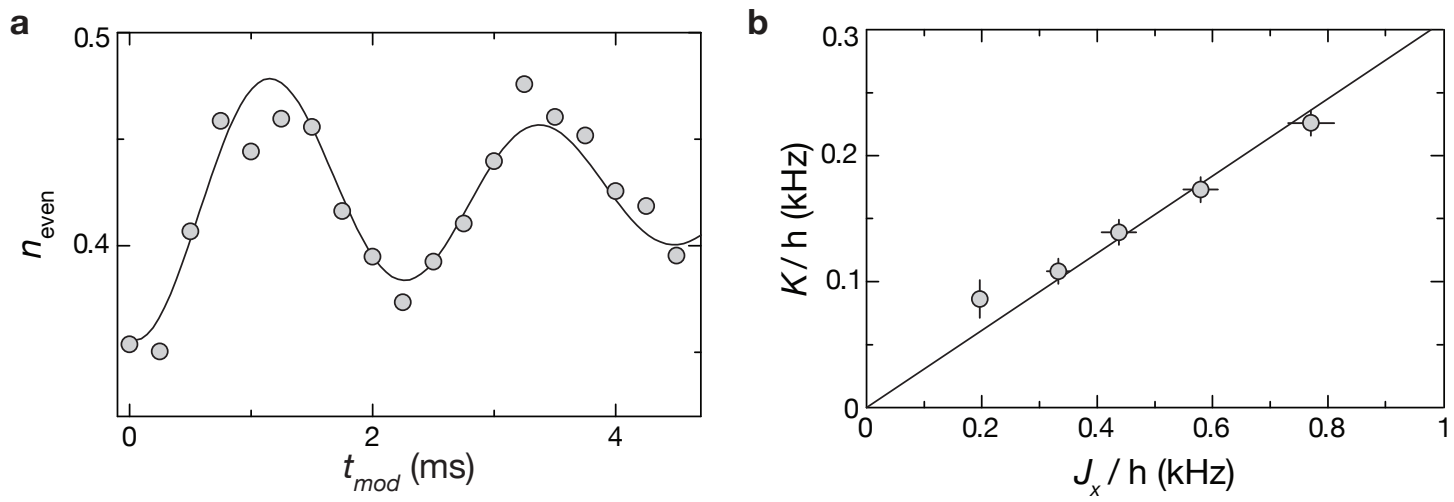

Figure 4.6: Calibration of the effective tunnel coupling induced by the laser-assisted tunneling method introduced in Sect. 5.3. a Rabi oscillations between odd and even sites triggered by the lattice modulation as a function of the modulation time $t_{\text {mod }}$ for $J_{x} / h=0.77(4) \mathrm{kHz}$. The solid line is a damped-sine fit to extract the oscillation frequency $\omega_{K}=2 K / \hbar$ which corresponds to $K / h=0.23(1) \mathrm{kHz}$. $\mathbf{b}$ Measured effective inner-well coupling $K$ as a function of the bare tunnel coupling $J_{x}$. The solid line is a linear fit to our data, where the offset was fixed to zero. The horizontal error bars depict the uncertainty in the calibration of $J_{x}$, which is mainly determined by the uncertainty of the lattice-depth calibration. The vertical error bars show the fit error obtained from the damped-sine fits as depicted in (a). (Figure adapted from Ref. [69])

Eq. (3.81).

The experimental sequence started by loading a BEC within $200 \mathrm{~ms}$ into a 3D optical lattice in the Mott-insulating (MI) regime [44] with $V_{x l}=35(1) \mathrm{E}_{\mathrm{rl}}, V_{y}=30(1) \mathrm{E}_{\mathrm{rs}}$ and $V_{z}=30(1) \mathrm{E}_{\mathrm{z}}$. The atoms were then loaded into the odd sites of the double-well potential by ramping up the short lattice along $x$ within $10 \mathrm{~ms}$ to its final value, which was varied in the range $V_{x}=8.5(1)-13.5(1) \mathrm{E}_{\mathrm{rs}}$. The relative phase was chosen in order to create a tilted double-well potential (Fig. 4.5a). The energy offset $\Delta / h \approx 4.4 \mathrm{kHz}$ was calibrated independently for each potential configuration $V_{x}$ by performing spectroscopy measurements as described in the previous section. The energy offset between neighboring sites was much larger than the coupling strength $\Delta \gg J_{x}$ such that all atoms initially populate odd sites and tunneling to even sites is inhibited. After instantaneously switching on the resonant modulation, atoms undergo tunnel oscillations between even and odd sites (Fig. 4.6a). To determine the effective coupling strength we measured the fraction of atoms in even sites $n_{\text {even }}$ as a function of the modulation time $t_{\text {mod }}$. The populations oscillate with frequency $\omega_{K}=2 \mathrm{~K} / \hbar$.

Ideally the oscillations would start at $n_{\text {even }}=0$ with an oscillation amplitude of one. The reduced contrast is most likely due to an imperfect initial state preparation and different atom numbers inside the double-wells. The signal can be improved by applying a filtering sequence as introduced in the following section, where all atoms on doubly occupied sites are removed from the trap. The damping of the oscillations is most likely due to inhomogeneities caused by the harmonic trap which leads to a dephasing of the 

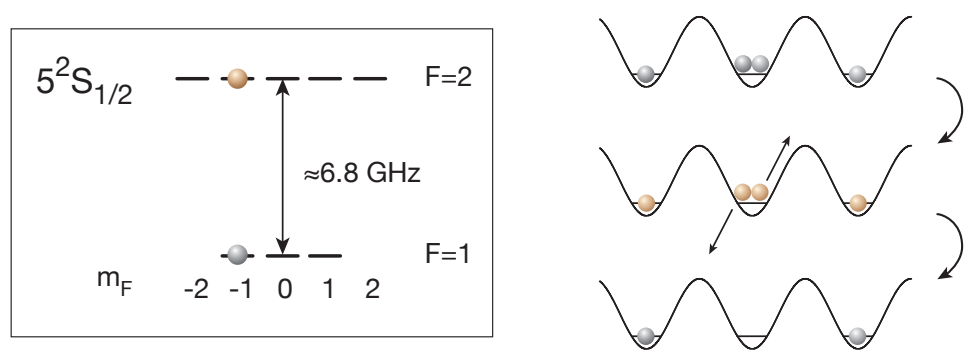

Rapid adiabatic passage

to $\left|F=2, m_{F}=-1\right\rangle$

Spin relaxation collisions

Rapid adiabatic passage to $\left|F=1, m_{F}=-1\right\rangle$

Figure 4.7: Schematic drawing of the filtering sequence used to remove atoms on doubly occupied sites. The filtering is applied on a MI state with at most two atoms per lattice site. The sequence starts by transferring all atoms from the $\left|F=1, m_{F}=-1\right\rangle$ state to the $\left|F=2, m_{F}=-1\right\rangle$ state. In this state atoms on doubly occupied sites undergo spin relaxation collisions. After a waiting time of typically $50 \mathrm{~ms}$ all double occupancies are removed from the trap. At the end the atoms are transferred back to the $\left|F=1, m_{F}=-1\right\rangle$ state.

oscillations in the individual double-well potentials.

The induced inner-well coupling $K$ was further measured as a function of the bare coupling $J_{x}$ (Fig. 4.6b). In agreement with theory, $K=J_{x} \mathcal{J}_{1}\left(\sqrt{2} V_{0} / \Delta\right)$, we found a linear relation between the induced and the bare tunnel coupling, where the proportionality constant $K / J_{x}=0.31$ (1) was evaluated from the linear fit depicted in (Fig. 4.6b). The modulation amplitude $V_{0}$ was additionally calibrated by loading the atoms into the diagonal lattice created by the two running-wave beams for $\omega=0$ using parametric heating [166]. The corresponding modulation amplitude was $V_{0}=2.1(1) \mathrm{E}_{\mathrm{rl}}$, which results in $\mathcal{J}_{1}\left(\sqrt{2} V_{0} / \Delta\right)=0.32(1)$ in agreement with the value extracted from the data shown in (Fig. 4.6b). For very small coupling strengths the damping time is on the order of the oscillation period, which is most likely the reason for the larger deviation of the measured coupling strength at $J_{x} / h=0.20(1) \mathrm{kHz}$ (Fig. 4.6b).

\subsection{Filtering sequence}

For some of the experiments it is required to prepare the atoms in a three dimensional optical lattice with at most one atom per lattice site. This can be achieved by loading a MI state where the inner-most shell has a density of $n=1$ atoms per lattice site. However, this regime crucially depends on the atom number and the external trapping potential. In order to be more flexible regarding the experimental parameters we apply a filtering sequence which allows us to remove possible double occupancies from the trap (Fig. 4.7).

The experimental sequence starts by loading a MI state within $100-200 \mathrm{~ms}$ in a parameter regime with at most two atoms per lattice site. The final lattice depths are typically about $20-30 \mathrm{E}_{\mathrm{ri}}, i=\{z, s, l\}$. After that the potential depths are ramped up 


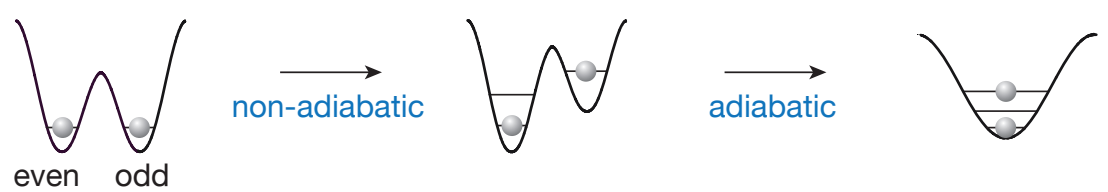

Figure 4.8: Schematic drawing of the site-resolved detection sequence in an isolated doublewell potential. Initially the atoms are located in even and odd sites of the double-well potential. Tunnel between neighboring sites is inhibited due to large potential barriers. As a first step the atoms in odd sites are transferred non-adiabatically to the third energy level of the doublewell potential by rapidly increasing the energy offset between neighboring sites. Then, the short lattice is removed adiabatically thereby preserving the band populations. Atoms initially located in odd sites are transferred to the third Bloch band in the long lattice and atoms initially in even sites stay in the lowest Bloch band.

to about $70-120 \mathrm{E}_{\mathrm{ri}}$ within $1 \mathrm{~ms}$ in order to increase the confinement and enhance the collision rate. At this point the atoms are transferred from the $\left|F=1, m_{F}=-1\right\rangle$ state to the $\left|F=2, m_{F}=-1\right\rangle$ state using a rapid adiabatic passage: In the presence of a microwave field at $v_{M W} \approx 6.8 \mathrm{GHz}$, which corresponds to the hyperfine-splitting of the ground state, a homogeneous offset magnetic field is swept across the resonance condition. The sweep rate is small enough to stay adiabatic and allows us to achieve an almost complete transfer to the $\left|F=2, m_{F}=-1\right\rangle$ state. In the $F=2$ manifold atoms can undergo hyperfine-changing collisions $|F=2\rangle \rightarrow|F=1\rangle$ leading to a decreased lifetime of atoms in the upper hyperfine manifold [167-169]. If two atoms collide at least one of them falls down to the $F=1$ manifold and an excess energy of about $h \times 6.8 \mathrm{GHz}$ is released. This is enough to expel both atoms from the trap. After a waiting time of $50 \mathrm{~ms}$ all atoms on doubly occupied sites are removed from the trap. The remaining atoms are transferred back to the $\left|F=1, m_{F}=-1\right\rangle$ state and we are left with at most one atom per lattice site.

\subsection{Site-resolved detection}

The superlattice potential offers a variety of manipulation and detection techniques $[33,34]$. Here, we discuss the site-resolved detection technique which is used for many experiments presented in the context of this thesis. It relies on the transfer of atoms that are located in certain sites of the lattice to higher Bloch bands and performing a subsequent band-mapping sequence [32], which allows us to determine the band occupations after TOF.

The detection sequence starts by ramping up all lattices rapidly within $1 \mathrm{~ms}$ in order to freeze out all dynamics; the long lattices are typically increased to about $V_{l}=70 \mathrm{E}_{\mathrm{rl}}$ and the short lattices to about $V=40 \mathrm{E}_{\mathrm{rs}}$. Subsequently the tilt between neighboring sites is increased non-adiabatically such that atoms located on odd sites are transferred 
to the third energy level (Fig. 4.8). As a second step the barrier of the double-well potential is ramped down adiabatically to $V_{s}=0 \mathrm{E}_{\mathrm{rl}}$. Hence, atoms initially located in even sites stay in the lowest Bloch band of the long lattice and atoms initially in odd sites end up in the third Bloch band. The higher-band occupations can be detected by applying a subsequent band-mapping, where atoms in the $n$-th Bloch band are mapped to the $n$-th Brillouin zone, $n \in \mathbf{N}$. Figure 4.9 illustrates the connection between the sitepopulations and higher band-occupations if the sequence is applied along $x$ (Fig. 4.9a), along $y$ (Fig. 4.9b) or along both directions (Fig. 4.9c). This technique is applicable for a single superlattice potential along $x$ or $y$ as well as a 2D superlattice potential. If both superlattices are used at the same time a lattice potential with a unit cell that contains four non-equivalent sites is formed. The new unit cell is typically denoted as plaquette. In this potential configuration the detection sequence applied along the $x$-axis gives access to the populations on the left and right bond in the plaquette,

$$
n_{\text {left }}=\frac{N_{A}+N_{D}}{N}, \quad n_{\text {right }}=\frac{N_{B}+N_{C}}{N},
$$

while along $y$ it determines the populations on the upper and lower bond,

$$
n_{\text {down }}=\frac{N_{A}+N_{B}}{N}, \quad n_{\text {up }}=\frac{N_{C}+N_{D}}{N} .
$$

By performing the site-resolved detection along both axes simultaneously (Fig. 4.9c) all site occupations

$$
n_{\mathcal{R}}=\frac{N_{\mathcal{R}}}{N}, \quad \mathcal{R}=\{A, B, C, D\},
$$

can be determined; here $N$ is the total atom number and $N_{\mathcal{R}}$ is defined as the atom number on site $\mathcal{R}$ in the plaquette [170]. 
a $\mathbf{i}$

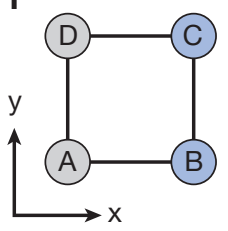

b i

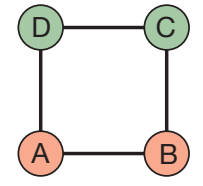

c i

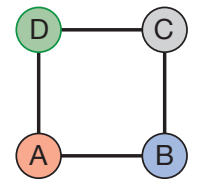

ii

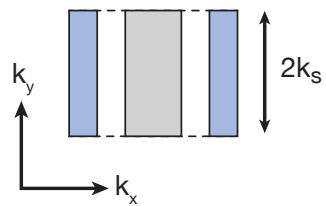

ii

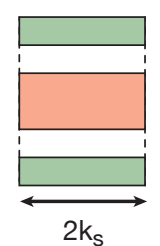

ii

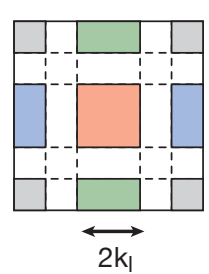

ii

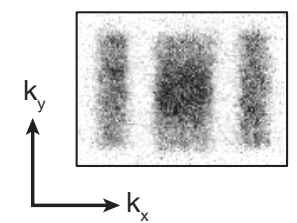

iii

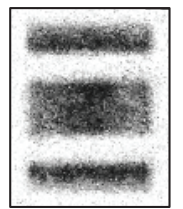

iii

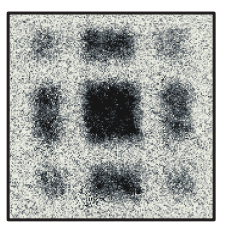

Figure 4.9: Schematic illustration of the connection between the site occupations and the corresponding Brillouin zones. i Illustration of the four non-equivalent lattice sites in the unitcell of a two-dimensional superlattice (plaquette). ii Schematic drawing of the Brillouin zones after applying the site-resolved detection technique $\mathbf{a}$ along $x, \mathbf{b}$ along $y$ or $\mathbf{c}$ along both directions simultaneously. iii Typical experimental images after applying the site-resolved detection along the corresponding directions measured after $10 \mathrm{~ms}$ TOF; atoms are shown in black. Applying the sequence along $x$ allows us to evaluate $n_{\text {left }}$ and $n_{\text {right }}$ and along $y$ to obtain $n_{\text {down }}$ and $n_{\text {up }}$. All site-populations $n_{\mathcal{R}}, \mathcal{R}=\{A, B, C, D\}$, can be detected simultaneously by applying the sequence along both directions at the same time. (Data in (a) and (b) taken from Ref. [70], data in (c) taken from Ref. [73]) 


\section{Chapter 5}

\section{Staggered magnetic flux}

The most straightforward implementation of artificial magnetic fields with laser-assisted tunneling (chapter 3) using the experimental apparatus described in the previous chapter is based on a staggered optical superlattice potential, which leads to a staggered effective flux distribution with zero mean $[68,69]$. The setup was inspired by the proposals of Jaksch \& Zoller [60] and subsequent work [61, 62], where complex hoppings arise due to coupling of the motional and internal degrees of freedom of the atoms. In contrast laser-assisted tunneling based on far-detuned running-wave beams does not rely on the internal structure of the atoms [63-65], which makes it applicable to a large variety of different systems. The resulting flux distribution alternates in sign along one of the lattice axis and is uniform along the other. It has been shown that such systems can feature interesting strongly-correlated phases as discussed in Ref. [171]. Other staggered flux distributions, arranged in a chequerboard-like pattern, were proposed using a time-dependent bichromatic optical potential [172]. These systems were studied theoretically in detail for Fermions, Bosons as well as Bose-Fermi mixtures [173-175]. Experimentally staggered fluxes were realized in triangular lattices, which served for simulations of magnetic systems [67, 125].

Here, we start with a theoretical analysis of the ground-state properties of the singleparticle Hamiltonian of a square lattice with staggered flux (Sect. 5.1). The degeneracy of the ground state depends on the field strength [171] as well as the tunnel couplings and can be probed experimentally by measuring the momentum distribution (Sect. 5.5). This experimental observable is indeed gauge-dependent (Sect. 5.2). In the experiment, the gauge is determined by the phases that are imprinted by the laser-assisted tunneling scheme. The configuration chosen in our setup [68, 69] and a detailed derivation of the corresponding Floquet Hamiltonian is given in Sect. 5.3 and 5.4, respectively. In addition, direct measurements of the flux are presented, which were obtained by partitioning the lattice into isolated four-site square plaquettes and detecting the evolution of single atoms in these plaquettes (Sect. 5.6). A theoretical discussion of the corresponding full-time dynamics can be found in Sect. 5.7. 


\subsection{Single-particle Hamiltonian}

The single-particle energy spectrum of a square lattice with staggered magnetic flux is studied as a function of the anisotropy in the tunnel couplings along $x$ and $y$ and the strength of the magnetic flux $\alpha$. The generating vector potential can be chosen similar to the usual Landau gauge (Sect. 2.3), such that the phase increases linearly along $y$ and alternates in sign along $x$. This system can be described by the following tight-binding Hamiltonian

$$
\begin{aligned}
& \hat{H}_{\text {stagg }}=-K \sum_{m, n} \mathrm{e}^{i \phi_{m, n}} \hat{a}_{m+1, n}^{+} \hat{a}_{m, n}-J \sum_{m, n} \hat{a}_{m, n+1}^{+} \hat{a}_{m, n}+\text { h.c. } \\
& \phi_{m, n}=2 \pi \alpha n(-1)^{(m+1)}
\end{aligned}
$$

where $K$ and $J$ are the coupling strengths along $x$ and $y$ respectively and tunneling along $x$ further involves the spatially-dependent phase factors $\phi_{m, n}$ (Fig. 5.1a). These phases give rise to a staggered magnetic field $\Phi_{m, n}=2 \pi \alpha(-1)^{m}$ which is uniform along $y$ and staggered along $x$ with zero mean (Fig. 5.1b) according to the definition in Eq. (3.82). The presence of the Peierls phase-factors breaks the translation symmetry of the underlying lattice and one needs to find the new magnetic translation symmetries of the Hamiltonian. As shown in Sect. 2.2 the flux through one magnetic unit cell equals an integer multiple of $2 \pi$. Hence, for the staggered flux lattice the smallest possible magnetic unit cell consists of two lattice unit cells, one with flux $\Phi_{m, n}=+2 \pi \alpha$ and one with $\Phi_{m, n}=-2 \pi \alpha$ (gray shaded area in Fig. 5.1b); it encloses an area that is pierced by a total flux $\Phi_{M U}=0$. The magnetic unit cell is two times larger than the normal lattice unit cell irrespective of the value of $\alpha$ and contains two non-equivalent sites, which we denote as odd and even.

The magnetic translation operators can be found in a similar way as described in Sect. 2.2. Note, however, that the phases $\theta_{m, n}^{i}$ are not directly determined by Eq. (2.9). Instead they can be derived following the calculations in Appendix A, Eqs. (A.1)-(A.4), by incorporating a priori the knowledge about the dimensions of the magnetic unit cell $A_{M U}=2 a \times 1 a$. As a result one obtains

$$
\theta_{m, n}^{x}=0, \quad \theta_{m, n}^{y}= \begin{cases}-2 \pi \alpha, & \text { for } m \text { odd } \\ 0, & \text { for } m \text { even }\end{cases}
$$

and

$$
\hat{M}_{x}^{2}=\sum_{m, n} \hat{a}_{m+2, n}^{\dagger} \hat{a}_{m, n}, \quad \hat{M}_{y}^{1}=\sum_{m, n} \hat{a}_{m, n+1}^{\dagger} \hat{a}_{m, n}\left\{\begin{array}{ll}
e^{-i 2 \pi \alpha}, & \text { for } m \text { odd } \\
1, & \text { for } m \text { even }
\end{array},\right.
$$

where $\hat{M}_{x}^{2}$ describes a translation by two lattice constants along $x$ and $\hat{M}_{y}^{1}$ a translation of one lattice constant along $y$. Both operators commute with the Hamiltonian as well 
a

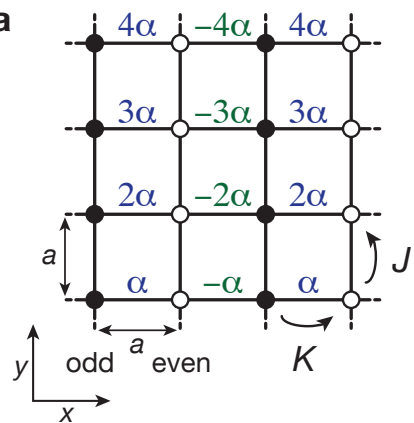

b

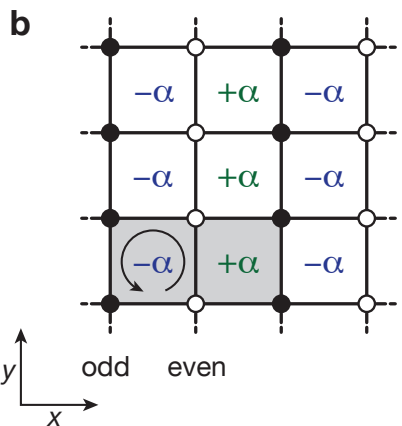

Figure 5.1: Peierls phase-factors $\phi_{m, n}$ and corresponding flux distribution $\Phi_{m, n}$ for a square lattice with staggered flux. a Phase distribution in units of $2 \pi$ as defined in Eq. (5.2). Along $y$ atoms can tunnel with amplitude $J$, while along $x$ the coupling strength is complex $K e^{i \phi_{m, n}}$ and depends on the position in the lattice. The lattice constant is denoted by $a$. $\mathbf{b}$ Illustration of the flux distribution $\Phi_{m, n}=2 \pi \alpha(-1)^{m}$ associated with Hamiltonian (5.1). The magnetic unit cell (gray shaded area) is twice as large as the normal lattice unit cell independent of $\alpha$ and contains two non-equivalent sites (odd and even).

as with each other. The simultaneous eigenstates which fulfill the generalized Bloch theorem in Eq. (2.18) can be written in the following form

$$
\Psi_{m, n}=\mathrm{e}^{i k_{x} m a} \mathrm{e}^{i k_{y} n a} \begin{cases}\psi_{0} \mathrm{e}^{i 2 \pi \alpha n}, & \text { for } m \text { odd } \\ \psi_{e}, & \text { for } m \text { even }\end{cases}
$$

where $k_{x}$ and $k_{y}$ are defined within the first magnetic Brillouin zone (FBZ), $-k_{s} / 2 \leq$ $k_{x}<k_{s} / 2$ and $-k_{s} \leq k_{y}<k_{s}$ and $k_{s}=\pi / a$. Inserting this ansatz into the Schrödinger equation associated with Hamiltonian (5.1)

$$
E \Psi_{m, n}=-K\left(\mathrm{e}^{i \phi_{m, n}} \Psi_{m+1, n}+\mathrm{e}^{-i \phi_{m-1, n}} \Psi_{m-1, n}\right)-J\left(\Psi_{m, n+1}+\Psi_{m, n-1}\right),
$$

the problem reduces to a two-dimensional eigenvalue equation

$$
H(\mathbf{k})\left(\begin{array}{l}
\psi_{e} \\
\psi_{0}
\end{array}\right)=E\left(\begin{array}{l}
\psi_{e} \\
\psi_{0}
\end{array}\right)
$$

with

$$
H(\mathbf{k})=-2\left(\begin{array}{cc}
J \cos \left(k_{y} a\right) & K \cos \left(k_{x} a\right) \\
K \cos \left(k_{x} a\right) & J \cos \left(k_{y} a+2 \pi \alpha\right)
\end{array}\right) .
$$

\section{Energy spectrum}

Due to the larger size of the magnetic unit cell, the unperturbed tight-binding band splits into two subbands (Fig. 5.2a). The bands touch at two Dirac cones in the FBZ 
a

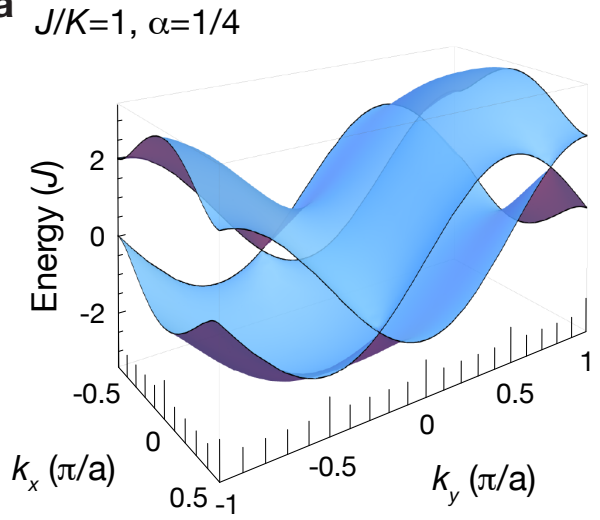

b

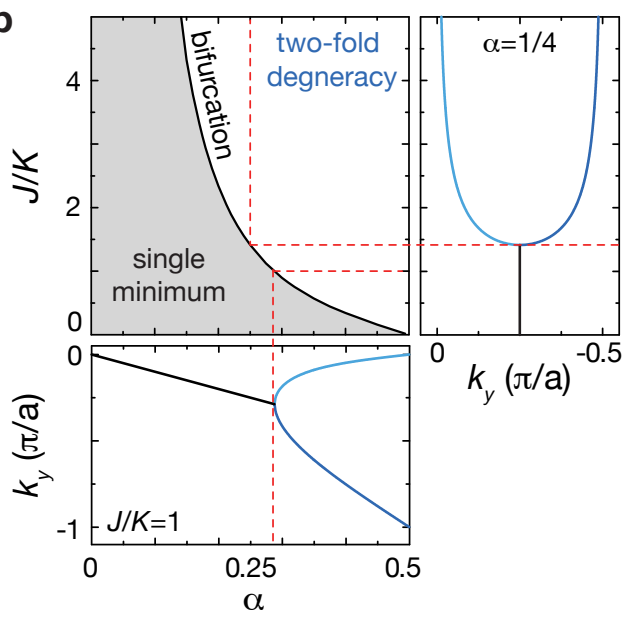

Figure 5.2: Single-particle energy spectrum and ground states. a Energy spectrum for $J / K=1$ and $\alpha=1 / 4$. The spectrum displays two subbands, that touch at the two Dirac cones at the border of the magnetic Brillouin zone. $\mathbf{b}$ Number of ground states as a function of the flux per plaquette $\alpha$ and coupling ratio $J / K$. Below a critical value of the flux, that depends on the coupling ratio, there is a single minimum in the lowest band (gray shaded area). Above that value the minimum splits into two and the spectrum exhibits two degenerate ground states. The lower panel shows the momentum component along $y$ of the lowest energy state for $J / K=1$ and the panel on the right for $\alpha=1 / 4$.

located at $\left(k_{x}, k_{y}\right)=(0.5,-\alpha) \pi / a$ and $\left(k_{x}, k_{y}\right)=(0.5,1-\alpha) \pi / a$. Their positions only depend on $\alpha$ but not on the coupling ratio $J / K$.

The lowest energy band exhibits either one or two minima within the FBZ depending on the flux $\alpha$ and $J / K$ (Fig. 5.2b). The momentum component along $x$ of the lowest energy state is always located at $k_{x}=0$, independent of $J / K$ or $\alpha$. Along $y$, however, the spectrum exhibits either a single or a two-fold degenerate minimum.

For isotropic coupling $J / K=1$ and below a critical value of the flux $\alpha<\alpha_{c} \simeq 0.29$ the lowest band exhibits a single minimum at $k_{y}=-\pi \alpha / a$. For larger values $\alpha>\alpha_{c}$ the minimum splits and there are two degenerate ground states in the system (lower panel in Fig. 5.2b). The critical value of the flux at which the bifurcation point occurs depends on the coupling ratio. The function $\alpha_{c}(J / K)$ is depicted with the solid black line in the upper left panel of Fig. 5.2b.

The right panel in Fig. 5.2b shows the momentum components of the ground state for constant flux $\alpha=1 / 4$. This situation was further studied experimentally in Sect. 5.5. Below a critical value $(J / K)_{c} \simeq 1.41$ the band exhibits a single minimum at $k_{y}=-\pi /(4 a)$, while above that value the minimum splits into two. In the limit $J \gg K$ the momenta approach the values $k_{y}=0$ and $k_{y}=-\pi /(2 a)$.

The absolute positions of the ground-state momentum components $\left(k_{x}, k_{y}\right)$ and the position of the Dirac cones are in general gauge-dependent. This has to be considered in 
cold-atom experiments, where the momentum distribution can be observed in TOF experiments [29]. The ground-state properties in the presence of interactions are discussed in Ref. [171] for isotropic couplings $J / K=1$.

\subsection{Gauge-dependence of expansion images}

In classical electromagnetism a gauge transformation is of the form $\mathbf{A} \rightarrow \mathbf{A}^{\prime}=\mathbf{A}-\nabla f$, where $\mathbf{A}$ is the vector potential generating a magnetic field $\mathbf{B}=\nabla \times \mathbf{A}$ and $f$ is a scalar field that depends on time and position. This transformation leaves all fields and therefore the Maxwell's equations invariant

$$
\mathbf{B}^{\prime}=\nabla \times \mathbf{A}^{\prime}=\nabla \times \mathbf{A}-\underbrace{\nabla \times \nabla f}_{=0}=\mathbf{B} .
$$

On a lattice with vector potential $\mathbf{A}_{m, n}=\left(A_{m, n}^{x}, A_{m, n}^{y}\right)$ a discrete version of the gauge transformation can be defined [171, 176, 177]

$$
\begin{aligned}
& A_{m, n}^{x} \rightarrow A_{m, n}^{\prime x}=A_{m, n}^{x}-\Delta_{x} f_{m, n} \\
& A_{m, n}^{y} \rightarrow \quad A_{m, n}^{\prime y}=A_{m, n}^{y}-\Delta_{y} f_{m, n}
\end{aligned}
$$

where $\Delta_{i}, i=\{x, y\}$ is the discrete form of the derivative

$$
\Delta_{x} f_{m, n}=f_{m+1, n}-f_{m, n}, \quad \Delta_{y} f_{m, n}=f_{m, n+1}-f_{m, n},
$$

and $f_{m, n}$ is a scalar function. The vector potential $\mathbf{A}_{m, n}$ gives rise to complex tunnel couplings as discussed in Sect. 2.1. For the sake of simplicity all pre-factors such as the electric charge $q$ as well as $\hbar$ are set to one such that the tight-binding Hamiltonian is given by the following expression

$$
\hat{H}=-J \sum_{m, n}\left(\mathrm{e}^{i A_{m, n}^{x}} \hat{a}_{m+1, n}^{\dagger} \hat{a}_{m, n}+\mathrm{e}^{i A_{m, n}^{y}} \hat{a}_{m, n+1}^{\dagger} \hat{a}_{m, n}+\text { h.c. }\right),
$$

and the flux per unit cell is determined by

$$
\Phi_{m, n}=A_{m, n}^{x}+A_{m+1, n}^{y}-A_{m, n+1}^{x}-A_{m, n}^{y} .
$$

The explicit form of Hamiltonian (5.12) depends on the gauge. The flux per unit cell, however, is gauge independent. For the transformed vector potential $\mathbf{A}_{m, n}^{\prime}$ it is given by

$$
\begin{aligned}
\Phi_{m, n}^{\prime}= & A_{m, n}^{x}+A_{m+1, n}^{y}-A_{m, n+1}^{x}-A_{m, n}^{y} \\
& \underbrace{-\left(f_{m+1, n}-f_{m, n}\right)-\left(f_{m+1, n+1}-f_{m+1, n}\right)+\left(f_{m+1, n+1}-f_{m, n+1}\right)+\left(f_{m, n+1}-f_{m, n}\right)}_{=0} .
\end{aligned}
$$



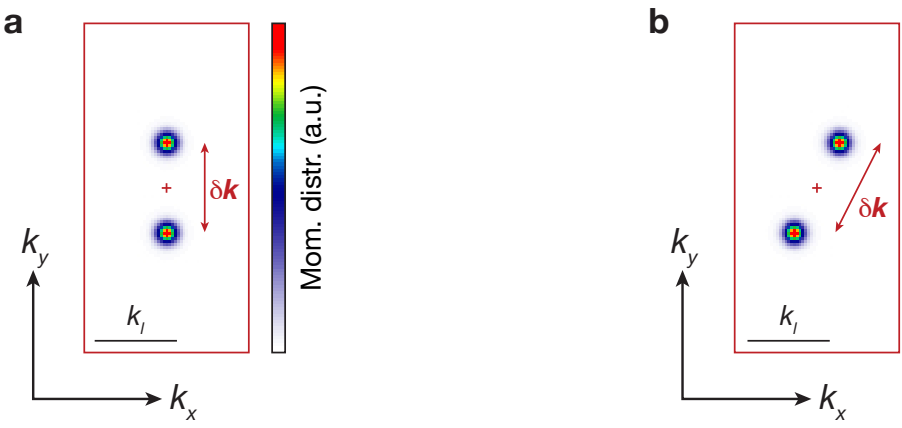

Figure 5.3: Momentum distribution in the FBZ of the ground state of Hamiltonian (5.1) for two different gauge fields that result in the same flux distribution. The distributions were calculated for isotropic coupling $J / K=1$ and two different phase distributions for fixed Wannier functions: a $\phi_{m, n}=(-1)^{m+1} n \pi / 2$ and $\mathbf{b} \phi_{m, n}=(-1)^{m+1}(n+m / 2) \pi / 2$. Both distributions give rise to the same flux distribution $\Phi_{m, n}=(-1)^{m} \pi / 2$ but the momentum distribution obtained in a TOF experiment differs due to the gauge-dependence of the corresponding observable. In both cases there are two momentum components in the FBZ at non-zero momentum and $\delta k_{y}=\pi /(2 a)$ (red arrows). The red crosses mark the center of the FBZ at zero momentum. The phases $\phi_{m, n}$ associated with the momentum distribution shown in (a) do not depend on the site index $m$. Therefore there is no displacement of the momentum components along $x$ so that $\delta k_{x}=0$. In contrast, the one associated with (b) does depend on $m$, which is the reason why the momenta additionally split along $x$ with $\delta k_{x}=\pi /(4 a)$. The ratio of the shift in momentum space $\delta k_{x} / \delta k_{y}=1 / 2$ is consistent with the spatial dependence of the phase $\phi_{m, n}$. The momentum distribution was obtained by exact diagonalization of a $31 \times 31$ matrix.

This proves the gauge invariance of the flux distribution $\Phi_{m, n}=\Phi_{m, n}^{\prime}$. Hamiltonian (5.12) can be brought back to its original form by redefining the creation and annihilation operators according to

$$
\hat{a}_{m, n} \rightarrow \hat{a}_{m, n}^{\prime}=\mathrm{e}^{i f_{m, n}} \hat{a}_{m, n}, \quad \hat{a}_{m, n}^{\dagger} \rightarrow \hat{a}_{m, n}^{\prime \dagger}=\mathrm{e}^{-i f_{m, n}} \hat{a}_{m, n}^{\dagger}
$$

Note that additional terms in the Hamiltonian that are proportional to the number operator $\hat{n}_{m, n}=\hat{a}_{m, n}^{+} \hat{a}_{m, n}$, such as on-site interactions or on-site potentials, are not affected by this transformation. All physical observables that depend only on the flux distribution $\Phi_{m, n}$ are gauge invariant and cannot distinguish between $\hat{a}_{m, n}$ and $\hat{a}_{m, n}^{\prime}$. This is true for example for the density of the wave function or the energy spectrum, which is gauge invariant up to a global shift in momentum space.

In cold-atom experiments the ground state can be probed through expansion imaging, where the momentum distribution is measured after TOF. This quantity, in contrast, depends on the choice of the gauge as will be shown in the following. In such experiments a BEC is typically prepared in the lowest energy state of a certain Hamiltonian. After switching off all fields abruptly (lattice potentials, trapping potential, gauge fields) and if interactions between the atoms can be neglected, the atoms expand ballistically 
for a period $t_{\mathrm{TOF}}$. The density distribution obtained after this time corresponds to the momentum distribution of the particles in the lattice and is given by

$$
\begin{aligned}
n(\mathbf{r}) & =\left(\frac{m_{a t}}{\hbar t_{\mathrm{TOF}}}\right)^{3}|\tilde{\omega}(\mathbf{k})|^{2} G(\mathbf{k}), \\
G(\mathbf{k}) & =\sum_{m, n, m^{\prime}, n^{\prime}} \mathrm{e}^{i\left(k_{x} a\left(m-m^{\prime}\right)+k_{y} a\left(n-n^{\prime}\right)\right)}\left\langle\hat{a}_{m, n}^{\dagger} \hat{a}_{m^{\prime}, n^{\prime}}\right\rangle
\end{aligned}
$$

where $m_{a t}$ is the mass of the particle, $\tilde{\omega}(\mathbf{k})$ is the Fourier transform of the on-site or Wannier function and $G(\mathbf{k})$ is the Fourier transform of the single-particle density matrix $\left\langle\hat{a}_{m, n}^{+} \hat{a}_{m^{\prime}, n^{\prime}}\right\rangle[29,178,179]$. The momentum $\mathbf{k}$ is related to the position $\mathbf{r}$ according to $\mathbf{k}=m_{a t} \mathbf{r} /\left(\hbar t_{\mathrm{TOF}}\right)$. Applying the gauge transformation as defined in Eq. (5.15) the single-particle density matrix becomes

$$
\left\langle\hat{a}_{m, n}^{\dagger} \hat{a}_{m^{\prime}, n^{\prime}}\right\rangle \quad \rightarrow \quad\left\langle\hat{a}_{m, n}^{\prime+} \hat{a}_{m^{\prime}, n^{\prime}}^{\prime}\right\rangle=\mathrm{e}^{i\left(f_{m^{\prime}, n^{\prime}}-f_{m, n}\right)}\left\langle\hat{a}_{m, n}^{\dagger} \hat{a}_{m^{\prime}, n^{\prime}}\right\rangle .
$$

The gauge transformation affects the single-particle density matrix and therefore also the momentum distribution, which is obtained experimentally using expansion imaging [171]. Figure 5.3 depicts the momentum distribution in the FBZ for two different vector potentials which give rise to the same staggered flux distribution $\Phi_{m, n}=(-1)^{m} \pi / 2$.

\subsection{Experimental setup}

The experimental setup consists of a two-dimensional lattice potential created by two orthogonal standing waves of wavelength $\lambda_{s}=767 \mathrm{~nm}$. Along the $x$-direction an additional standing wave with twice the wavelength $\lambda_{l}=2 \lambda_{s}$ is superimposed to introduce a staggered energy offset $\pm \Delta / 2$ (Fig. 5.4a), where the convention is used that odd lattice sites have lower on-site energy than even lattice sites.

For $\Delta \gg J_{x}$ tunneling is inhibited along the $x$-direction, while bare tunneling occurs along $y$. A pair of running-wave beams ${ }^{1}$ with electric fields $\mathbf{E}_{i}(\mathbf{r}, t), i=\{1,2\}$, wave vectors $\left|\mathbf{k}_{1}\right| \simeq\left|\mathbf{k}_{2}\right| \equiv k_{R}$ and frequencies $\omega_{i}$, is then used to periodically drive the system and restore resonant tunneling for $\omega=\omega_{1}-\omega_{2}= \pm \Delta / \hbar$. Each of the two beams is aligned along one of the two lattice axis

$$
\mathbf{E}_{1}(\mathbf{r}, t)=E_{0}^{x} \mathrm{e}^{i\left(k_{1} x+\omega_{1} t+\phi_{1}\right)}, \quad \mathbf{E}_{2}(\mathbf{r}, t)=E_{0}^{y} \mathrm{e}^{i\left(k_{2} y+\omega_{2} t+\phi_{2}\right)},
$$

as illustrated in Fig. 5.4b. The corresponding local optical potential is of the following form

$$
V(\mathbf{r}, t)=\underbrace{\left|V_{0}^{x}\right|^{2}+\left|V_{0}^{y}\right|^{2}}_{\text {constant }}+\underbrace{V_{0} \cos \left(\delta \mathbf{k} \cdot \mathbf{r}+\omega t+\phi_{0}\right)}_{=V_{K}(t)},
$$

\footnotetext{
${ }^{1}$ Erbium doped fiber amplifier from NP Photonics (output power: $5 \mathrm{~W}$ )
} 
a

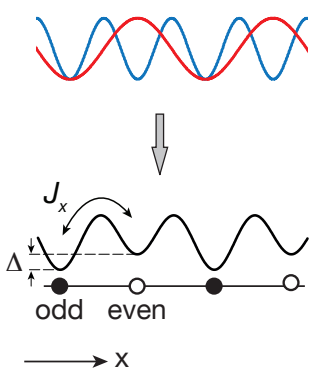

b

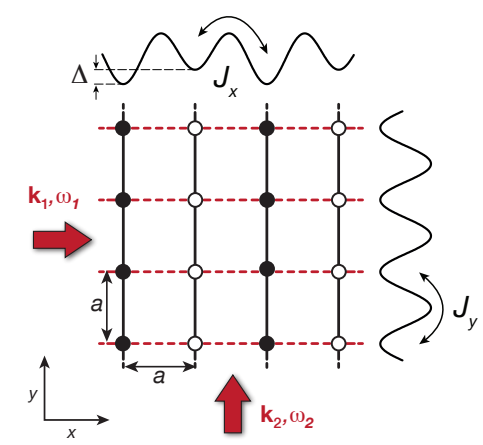

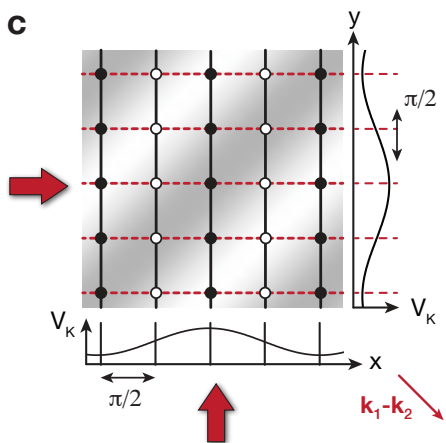

Figure 5.4: Experimental setup for the realization of a staggered artificial magnetic field. a Schematic drawing of the staggered lattice potential along the $x$-direction with energy offset $\Delta$ and tunnel coupling $J_{x}$ between neighboring sites. It consists of two standing waves with wavelength $\lambda_{s}=767 \mathrm{~nm}$ (blue) and $\lambda_{l}=2 \lambda_{s}$ (red). Odd lattice sites (black circles) denote sites of low on-site energy and even lattice sites (white circles) the ones of high energy. $\mathbf{b}$ Illustration of the full two-dimensional lattice with lattice constant $a=\lambda_{s} / 2$ and tunnel coupling $J_{y}$ along $y$. An additional pair of running-wave beams (red arrows) with wave vectors $\mathbf{k}_{i}, i \in\{1,2\}$ and frequencies $\omega_{i}$ are used to periodically modulate the lattice potential. c Local optical potential created by the interference between the two running-wave beams for a fixed time $t$. The phase fronts determine the site-dependent phases $\varphi_{m, n}$ of the time-dependent on-site potential.

with $\delta \mathbf{k}=\mathbf{k}_{1}-\mathbf{k}_{2}, \phi_{0}=\phi_{1}-\phi_{2}$ and the potential depth is proportional to the amplitude of the electric fields $V_{0}^{i} \propto\left|E_{0}^{i}\right|^{2}$ and $V_{0} \propto 2 E_{0}^{x} E_{0}^{y}$ [158]. The static term leads to a global shift of the potential energy and can be neglected. Only the time-periodic on-site modulation $V_{K}(t)$ is relevant. The site-dependent phases of this modulation are given by $\delta \mathbf{k} \cdot \mathbf{R}$, where $\mathbf{R}$ defines the position in the lattice, $\mathbf{R}=m a \hat{\mathbf{e}}_{x}+n a \hat{\mathbf{e}}_{y}$. This expression can be recast into the form

$$
\delta \mathbf{k} \cdot \mathbf{R}=k_{R} a(m-n),
$$

For our experimental parameters $k_{R}=\pi /(2 a)$ such that the phases are determined by

$$
\delta \mathbf{k} \cdot \mathbf{R}=\frac{\pi}{2}(m-n)
$$

The phase difference of the modulation between neighboring sites along the two directions is given by $\delta \varphi_{x}=-\delta \varphi_{y}=\pi / 2$ (Fig. 5.4c). By choosing a different geometry or a different wave vector $k_{R}$, in principle any other phase difference can be engineered. The additional phase $\phi_{0}$ in Eq. (5.19) is given by the relative phase between the two running-wave beams, which is not actively stabilized and differs between individual experimental realizations. 


\subsection{Effective Floquet Hamiltonian and micro-motion}

The dynamics of the system described in the previous section is governed by an explicitly time-dependent Hamiltonian

$$
\begin{aligned}
\hat{H}(t) & =\sum_{m, n}\left(-J_{x} \hat{a}_{m+1, n}^{\dagger} \hat{a}_{m, n}-J_{y} \hat{a}_{m, n+1}^{\dagger} \hat{a}_{m, n}+\text { h.c. }\right) \\
& +\sum_{m, n}\left(V_{0} \sin \left(\omega t+\varphi_{m, n}\right)+(-1)^{m} \frac{\Delta}{2}\right) \hat{n}_{m, n},
\end{aligned}
$$

with $\varphi_{m, n}=\frac{\pi}{2}(m-n+1)+\phi_{0}$ being the site-dependent phase generated through the application of the running-wave beams depicted in Fig. 5.4b. Since the Hamiltonian is periodic in time $\hat{H}(t+T)=\hat{H}(t)$, with $T=2 \pi / \omega$, Floquet's theorem applies. As discussed in Sect. 3.1 the evolution of the system over one period $T$ can be described by an effective time-independent Hamiltonian. The Hamiltonian above is of the form studied in Sect. 3.4 so that in the high frequency limit $\hbar \omega \gg J_{x}, J_{y}$ the formalism can be applied directly. The static terms, that are proportional to $\hbar \omega=\Delta$, diverge in the limit $\omega \rightarrow \infty$. Therefore a transformation into the rotating frame is performed as demonstrated in Sect. 3.4 using the unitary operator defined in Eq. (3.73) for $\Delta_{m}=(-1)^{m} \Delta / 2$,

$$
\hat{R}_{\text {stagg }}(t)=\exp \left[i \sum_{m, n}\left(-\frac{V_{0}}{\hbar \omega} \cos \left(\omega t+\varphi_{m, n}\right)+(-1)^{m} \frac{\Delta t}{2 \hbar}\right) \hat{n}_{m, n}\right] .
$$

This leads to the following transformed Hamiltonian, where the time-dependence appears in the tunnel couplings

$$
\begin{aligned}
\hat{\mathcal{H}}_{\text {stagg }}(t)= & -J_{x} \sum_{m, n}\left(\mathrm{e}^{i \eta_{m, n}^{x}(t)} \hat{a}_{m+1, n}^{\dagger} \hat{a}_{m, n}+\text { h.c. }\right) \\
& -J_{y} \sum_{m, n}\left(\mathrm{e}^{i \eta_{m, n}^{y}(t)} \hat{a}_{m, n+1}^{\dagger} \hat{a}_{m, n}+\text { h.c. }\right),
\end{aligned}
$$

with

$$
\begin{aligned}
& \eta_{m, n}^{x}(t)=-\eta_{0} \sin \left(\omega t+\frac{\varphi_{m+1, n}+\varphi_{m, n}}{2}\right)+(-1)^{m+1} \Delta t / \hbar, \\
& \eta_{m, n}^{y}(t)=\eta_{0} \sin \left(\omega t+\frac{\varphi_{m, n+1}+\varphi_{m, n}}{2}\right) .
\end{aligned}
$$

Since the phase difference of the modulation between neighboring sites along both directions is equal $\left|\delta \varphi_{x}\right|=\left|\delta \varphi_{y}\right|=: \delta \varphi=\pi / 2$, the differential modulation amplitude $\eta_{0}$ is also the same,

$$
\eta_{0}=2 \gamma \sin (\delta \varphi)=\frac{\sqrt{2} V_{0}}{\hbar \omega}
$$


For resonant driving $\hbar \omega=\Delta$ and $\hbar \omega \gg J_{x}, J_{y}$ the effective Floquet Hamiltonian in the rotating frame is well approximated by the lowest order of the Magnus expansion (Sect. 3.1.3), which results in the following Hamiltonian

$$
\hat{\mathcal{H}}_{F}^{\text {stagg }}=-K \sum_{m, n} \mathrm{e}^{i \phi_{m, n}} \hat{a}_{m+1, n}^{\dagger} \hat{a}_{m, n}-J \sum_{m, n} \hat{a}_{m, n+1}^{\dagger} \hat{a}_{m, n}+\text { h.c. },
$$

with $K=J_{x} \mathcal{J}_{1}\left(\eta_{0}\right)$ and $J=J_{y} \mathcal{J}_{0}\left(\eta_{0}\right)$. Due to the staggered energy offset $\Delta_{m}=$ $(-1)^{m} \Delta / 2$ the phases vary non-uniformly across the lattice (Sect. 3.2) and are determined by

$$
\phi_{m, n}= \begin{cases}-\left(\varphi_{m+1, n}+\varphi_{m, n}\right) / 2, & \text { for } m \text { odd } \\ +\left(\varphi_{m+1, n}+\varphi_{m, n}\right) / 2+\pi, & \text { for } m \text { even }\end{cases}
$$

\section{General form of the flux distribution}

The lattice contains two different kinds of plaquettes with fluxes $\Phi_{m, n}^{o}$ and $\Phi_{m, n}^{e}$ respectively (Fig. 5.5). For a positive energy offset between neighboring sites $\Delta_{m+1}-\Delta_{m}=\Delta$ ( $m$ odd), the Peierls phase-factor is given by $\tilde{\phi}_{m, n}:=-\left(\varphi_{m+1, n}+\varphi_{m, n}\right) / 2$. The corresponding flux through the lattice unit cell is defined as

$$
\Phi_{m, n}^{o}=\tilde{\phi}_{m, n}-\tilde{\phi}_{m, n+1} .
$$

For those plaquettes where the energy offset between neighboring sites is negative $\Delta_{m+1}-\Delta_{m}=-\Delta$ the Peierls phases change sign and are shifted by $\pi, \tilde{\phi}_{m, n} \rightarrow-\tilde{\phi}_{m, n}+\pi$. This is the case for $m$ even such that the corresponding flux is given by

$$
\Phi_{m, n}^{e}=-\tilde{\phi}_{m, n}+\tilde{\phi}_{m, n+1}=-\Phi_{m, n}^{o}
$$

This shows that laser-assisted tunneling using a staggered superlattice potential naturally gives rise to a staggered flux distribution.

\section{Gauge realized in the experiment}

For the laser-beam configuration depicted in Fig. 5.4b the phases of the on-site modulation are determined by $\varphi_{m, n}=\frac{\pi}{2}(m-n+1)+\phi_{0}$ so that the corresponding Peierls phases $\phi_{m, n}$ are given by

$$
\phi_{m, n}= \begin{cases}-\frac{\pi}{2}(m-n+3 / 2)-\phi_{0}, & \text { for } m \text { odd } \\ +\frac{\pi}{2}(m-n+7 / 2)+\phi_{0}, & \text { for } m \text { even }\end{cases}
$$

This precise form of the gauge will be important for the experiments where the momentum distribution is measured using expansion imaging (Sect. 5.5). As discussed in 

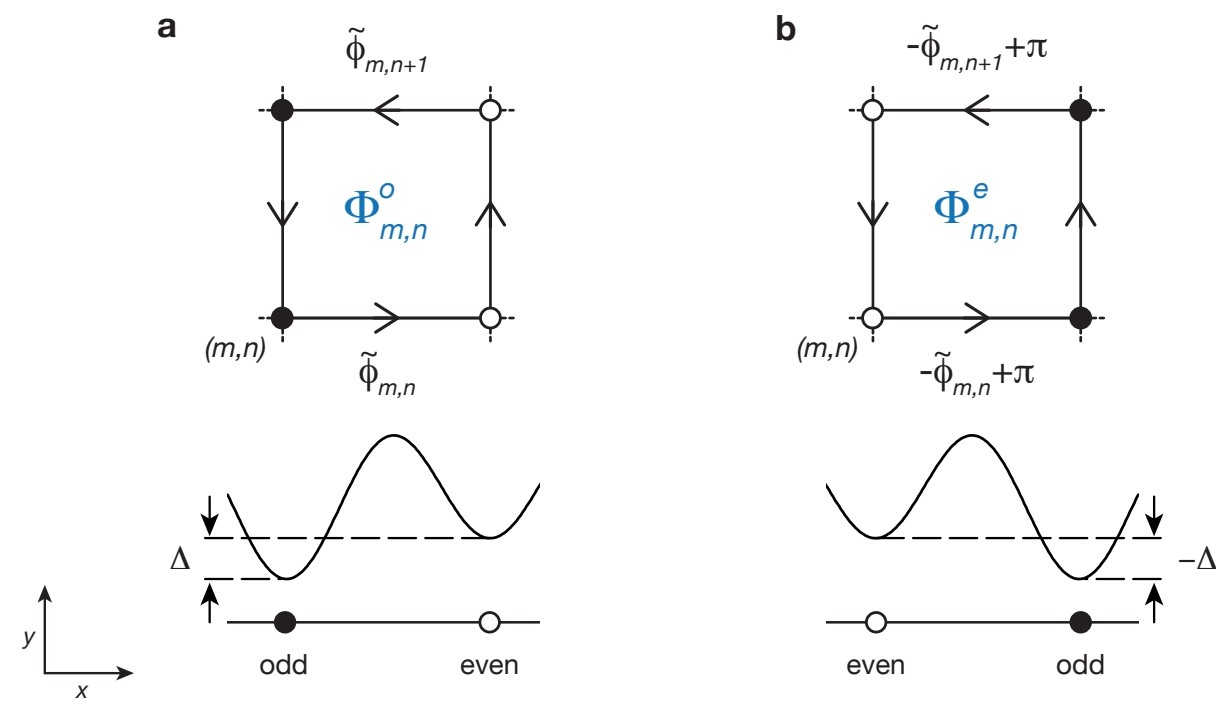

Figure 5.5: Schematic drawing of the two different plaquettes with fluxes $\Phi_{m, n}^{o}=-\Phi_{m, n}^{e}$ realized using a staggered superlattice potential. a For $m$ odd the energy offset between neighboring sites is positive $+\Delta$ and the flux through the plaquette is determined by $\Phi_{m, n}^{o}=$ $\tilde{\phi}_{m, n}-\tilde{\phi}_{m, n+1}$. b For $m$ even the offset is negative $-\Delta$ and the Peierls phases change sign $\tilde{\phi}_{m, n} \rightarrow-\tilde{\phi}_{m, n}+\pi$. The constant shift by $\pi$ does not depend on the site, hence, it cannot influence the value of the flux. As a result the flux per plaquette changes sign $\Phi_{m, n}^{e}=-\Phi_{m, n}^{o}$ compared to the one for $m$ odd.

Sect. 5.2 the particular choice of gauge manifests itself in the position of the momentum components. Since the experimental gauge is determined by the laser-beam configuration it is necessary to derive the effective Hamiltonian taking into account all relevant phase factor that might appear in the setup. Fortunately for the case of a staggered flux distribution the relative phase $\phi_{0}$ between the two running-wave beams, which is not stabilized in the experiment, has no impact on the momentum distribution (Appendix C). For the sake of simplicity we choose $\phi_{0}=-3 \pi / 4$ and rewrite the expression for the phases given in Eq. (5.32) as follows

$$
\phi_{m, n}=\frac{\pi}{2}(m+n)(-1)^{m+1} .
$$

The two expressions are equivalent as illustrated in Fig. 5.6 except that they appear to be shifted by two lattice constants along $y$ relative to each other. For the following discussion we are going to use the convention that the effective magnetic field is pointing along the $-\hat{e}_{z}$ direction such that the flux per lattice unit cell is given by

$$
\Phi_{m, n}=\frac{\pi}{2}(-1)^{m+1},
$$

which is uniform along $y$ and staggered along $x[68,69]$. An equivalent derivation of the effective Hamiltonian using the formalism developed in Sect. 3.1.2 can be found in 

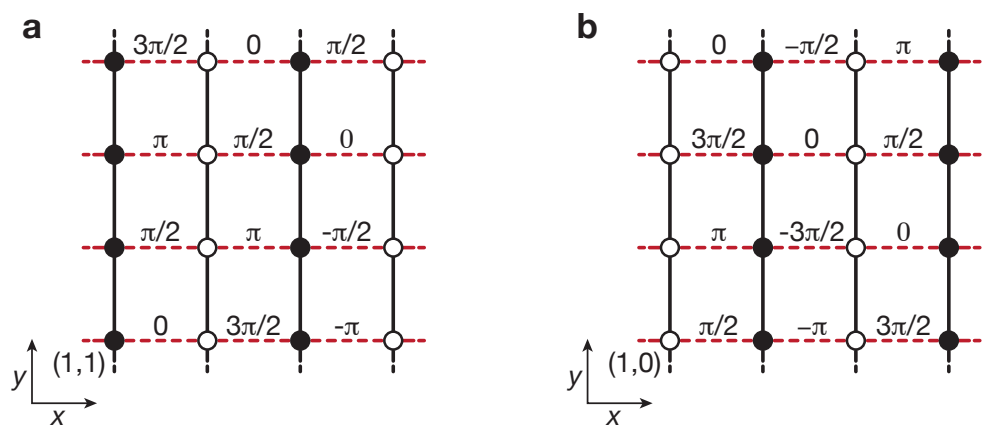

Figure 5.6: Schematic drawing of the vector potential realized in the experiment. a Phase distribution of the complex hopping as given in Eq. (5.32) for $\phi_{0}=-3 \pi / 4$. b The experimentally realized phase distribution can be represented by the equivalent expression $\phi_{m, n}=$ $\frac{\pi}{2}(m+n)(-1)^{m+1}$.

Ref. [66], which is in agreement with the results obtained using the Magnus expansion approach discussed above.

\section{Micro-motion operator}

Following the discussion of the micro-motion in 1D lattice geometries in Sect. 3.3 it is expected that the micro-motion also influences the momentum distribution in the staggered flux lattice. The full-time evolution according to Eqs. (3.39)-(3.40) is given by

$$
\hat{U}(t)=\exp \left[i \hat{\mathcal{M}}_{\text {stagg }}^{+}(t)\right] \exp \left[-\frac{i}{\hbar} \hat{\mathcal{H}}_{F}^{\text {stagg }} t\right] \exp \left[i \hat{\mathcal{M}}_{\text {stagg }}^{\dagger}(0)\right]
$$

where the micro-motion operator is determined be the unitary operator $\hat{R}_{\text {stagg }}(t)=$ $\exp \left[i \hat{\mathcal{M}}_{\text {stagg }}(t)\right]$ given in Eq. (5.23) such that

$$
\hat{\mathcal{M}}_{\text {stagg }}(t)=\sum_{m, n}\left(-\frac{V_{0}}{\hbar \omega} \cos \left(\omega t+\varphi_{m, n}\right)+(-1)^{m} \frac{\Delta}{2 \hbar} t\right) \hat{n}_{m, n}
$$

The micro-motion associated with this operator is very similar to the case of a periodically modulated 1D staggered superlattice potential. The part that is related to the staggered offset potential $\Delta_{m}=(-1)^{m} \Delta / 2$ is trivial and only leads to oscillations in the amplitudes of the different momentum components. The modulation-induced term, however, can lead to additional momentum components shifted by integer multiples of $\delta \mathbf{k}=(\pi /(2 a), \pi /(2 a))$. In the following section experimental signatures of these additional components will be revealed. 

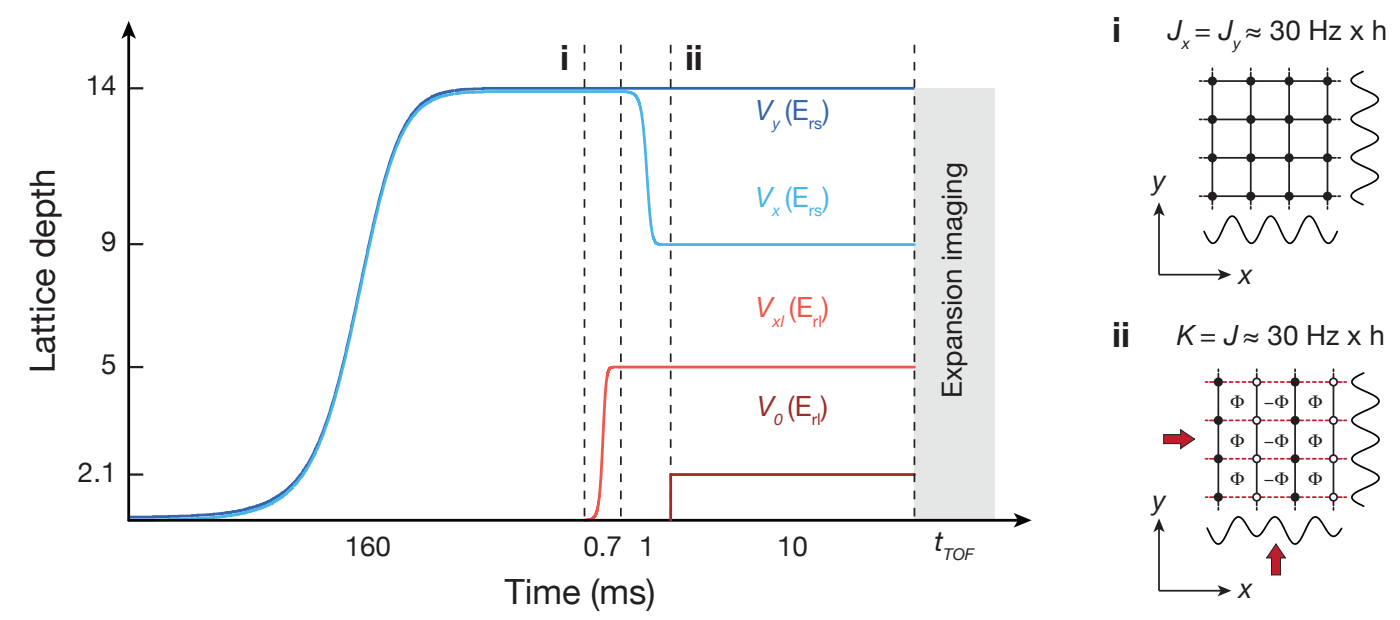

Figure 5.7: Schematic drawing of the experimental sequence. The atoms were loaded adiabatically within $160 \mathrm{~ms}$ into a two-dimensional lattice with isotropic tunnel coupling along both directions $J_{x} / h=J_{y} / h=31(2) \mathrm{Hz}$ as illustrated by the schematic drawing in (i). After switching on the staggered tilt $V_{x l}$ and decreasing the short lattice along $x$, spontaneous tunneling was inhibited by the staggered superlattice potential shown in (ii). Coupling was then restored by switching on the modulation $V_{0}$ (red arrows in ii). The modulation amplitude and lattice depths were chosen such that the final effective coupling strengths $K / h=J / h=30(2) \mathrm{Hz}$ matched the ones of the initial state. After an additional holding time of $10 \mathrm{~ms}$ coherence was restored and the momentum distribution was measured after $20 \mathrm{~ms}$ TOF.

\subsection{Ground state of the staggered flux lattice}

The ground state of the staggered flux lattice is studied experimentally for a fixed value of the flux $\Phi_{m, n}= \pm 2 \pi \alpha$, with $\alpha=1 / 4$, as a function of the coupling ratio $\mathrm{J} / \mathrm{K}$ using expansion imaging (Sect. 5.2). The properties of the ground state were studied theoretically at the beginning of this chapter. For $\alpha=1 / 4$ it was found that there is a bifurcation point at $(J / K)_{c} \simeq 1.41$, where the ground state of the system becomes two-fold degenerate. The staggered-flux Hamiltonian in the experimental gauge was derived in the previous section and can be written in the following form

$$
\begin{aligned}
& \hat{\mathcal{H}}_{F}^{\text {stagg }}=-K \sum_{m, n} \mathrm{e}^{i \phi_{m, n}} \hat{a}_{m+1, n}^{\dagger} \hat{a}_{m, n}-J \sum_{m, n} \hat{a}_{m, n+1}^{\dagger} \hat{a}_{m, n}+\text { h.c. } \\
& \phi_{m, n}=\frac{\pi}{2}(m+n)(-1)^{m+1},
\end{aligned}
$$

with $K=J_{x} \mathcal{J}_{1}\left(\eta_{0}\right), J=J_{y} \mathcal{J}_{0}\left(\eta_{0}\right)$ and $\eta_{0}=\sqrt{2} V_{0} /(\hbar \omega)$.

\subsubsection{Isotropic coupling $J=K$}

In order to prepare the ground state of the staggered-flux Hamiltonian we used the experimental sequence illustrated in Fig. 5.7. It started by loading a BEC of about $5 \times 10^{4}$ 


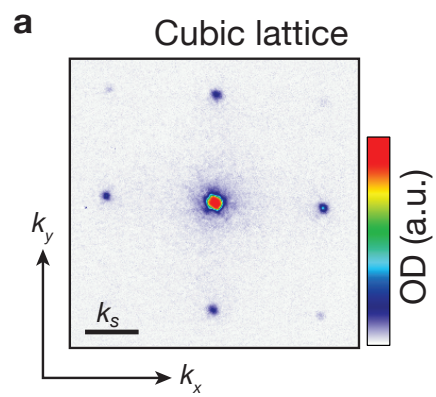

b

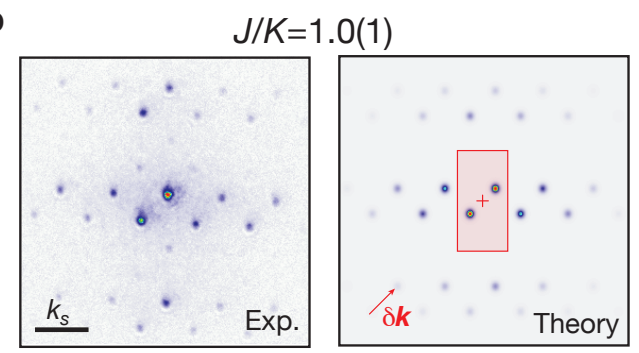

Figure 5.8: Momentum distribution measured after $t_{T O F}=20 \mathrm{~ms}$ for a simple cubic lattice and the staggered flux lattice with isotropic coupling. a The distribution was obtained by loading the atoms into the ground state of a simple cubic lattice with lattice constant $a=\lambda_{s} / 2$ without modulation $V_{0}=0$ and flux $\Phi=0$. b For the measured momentum distribution (left panel) the atoms were loaded into a staggered flux lattice with $\Phi=(-1)^{m+1} \pi / 2$ and $J / K=1.0(1)$. The theoretical momentum distribution (right panel) was obtained by an exact diagonalization of a $31 \times 31$ lattice with a harmonic confinement of $\omega_{x} /(2 \pi)=\omega_{y} /(2 \pi)=20 \mathrm{~Hz}$. The red rectangle marks the dimensions of the magnetic Brillouin zone and the cross marks the center position at zero momentum. There are two momentum components in the FBZ which are shifted by $\delta \mathbf{k}$ (red arrow). Each image was normalized to its maximum intensity. (Data taken from Ref. [68])

atoms without any discernible fraction of thermal atoms into a 2D optical lattice, which results in a 2D array of coupled 1D Bose gases. Perpendicular to the lattice the atoms were confined by a crossed dipole trap with a harmonic trapping potential of frequency $\omega_{z} /(2 \pi) \simeq 30 \mathrm{~Hz}$. The atoms were loaded adiabatically within $160 \mathrm{~ms}$ into the lattice with final depths $V_{x}=V_{y}=14(1) \mathrm{E}_{\mathrm{rs}}$ (see Sects. 4.2-4.3 for an overview of the lattice and superlattice potentials). The bare tunnel couplings along both directions correspond to $J_{x} / h=J_{y} / h=31(2) \mathrm{Hz}$ and were chosen to match the final effective coupling strengths $K$ and $J$ at the end of the sequence. After the initial loading the long lattice along $x$ was ramped up within $0.7 \mathrm{~ms}$ to $V_{x l}=5.0(2) \mathrm{E}_{\mathrm{rl}}$ in order to introduce a staggered energy offset between neighboring sites of $\Delta / h=4.4(1) \mathrm{kHz}$. This value was calibrated independently as described in Sect. 4.3.2. Subsequently the short lattice along $x$ was decreased to $V_{x}=9.0(3) \mathrm{E}_{\mathrm{rs}}$, which corresponds to a bare coupling strength $J_{x} / h=$ 94(4) Hz. Since $\Delta$ was much larger than the tunnel coupling $J_{x}$ all dynamics was frozen along $x$, while the atoms were free to tunnel along $y$. By switching on the modulation on resonance with amplitude $V_{0}=2.10(5) \mathrm{E}_{\mathrm{rl}}$ and frequency $\omega=2 \pi \times 4.4 \mathrm{kHz}$ tunneling along $x$ was restored. In the limit $\eta_{0} \ll 1$ the effective couplings can be approximated by $K \simeq J_{x} V_{0} /(\sqrt{2} \Delta)$ and $J \simeq J_{y}$ such that the effective coupling strength is given by $K / h=30(2) \mathrm{Hz}$ and the final coupling ratio is $J / K=1.0(1)$.

After holding the atoms in this configuration for $10 \mathrm{~ms}$ we observed a restored phase coherence (Fig. 5.8b) most likely due to a redistribution of entropy, which was introduced by the random phases between the one-dimensional condensates, along the longitudinal modes. For comparison the momentum distribution of a simple cubic lattice is depicted 

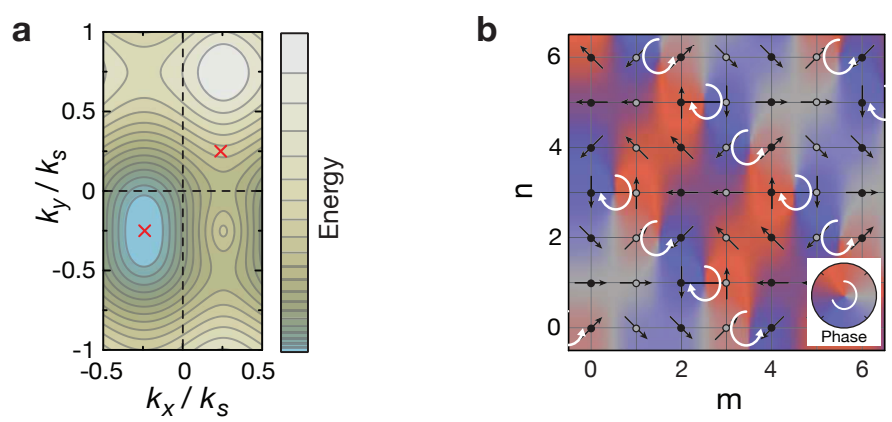

Figure 5.9: Dispersion relation and ground-state wave function for $J / K=1$. a The dispersion relation of the lowest band was calculated using Eq. (5.39). It exhibits a single minimum, which appears as two momentum components (red crosses) in the first magnetic Brillouin zone. $\mathbf{b}$ Spatial distribution of the phase of the ground-state wave function encoded in the direction of the black arrows. Their length illustrates the spatial distribution of the density, which is uniform. The background color further depicts the values of the phase interpolated between neighboring lattice sites. The position and chirality of the phase-vortices are illustrated with white arrows. (Data taken from Ref. [68])

in Fig. 5.8a. For this case a single momentum component appears in the FBZ at zero momentum $k_{x}=k_{y}=0$ together with additional momentum components separated by the reciprocal lattice vectors $\left(2 k_{s}, 0\right)$ and $\left(0,2 k_{s}\right)$, with $k_{s}=\pi / a$. In contrast to that there are four momentum components per cubic lattice Brillouin zone in the presence of the staggered magnetic flux (Fig. 5.8b). In order to understand this momentum distribution the Schrödinger equation has to be solved in the experimentally realized gauge since the position of the momentum components is gauge-dependent (Sect. 5.2). A detailed derivation can be found in Appendix C, where it is shown that the eigenstates can be written in the following form

$$
\Psi_{m, n}=\mathrm{e}^{i k_{x} m a} \mathrm{e}^{i k_{y} n a}\left\{\begin{array}{ll}
\psi_{0} \mathrm{e}^{i \frac{\pi}{2}(m+n)}, & \text { for } m \text { odd } \\
\psi_{e}, & \text { for } m \text { even }
\end{array} .\right.
$$

Inserting this ansatz into the Schrödinger equation associated with Hamiltonian (5.37) the problem reduces to the following two-dimensional eigenvalue equation

$$
\left(\begin{array}{cc}
-2 J \cos \left(k_{y} a\right) & -K\left(i \mathrm{e}^{i k_{x} a}+\mathrm{e}^{-i k_{x} a}\right) \\
-K\left(-i \mathrm{e}^{-i k_{x} a}+\mathrm{e}^{i k_{x} a}\right) & 2 J \sin \left(k_{y} a\right)
\end{array}\right)\left(\begin{array}{l}
\psi_{e} \\
\psi_{0}
\end{array}\right)=E(\mathbf{k})\left(\begin{array}{c}
\psi_{e} \\
\psi_{0}
\end{array}\right),
$$

where $k_{x}, k_{y}$ are defined within the FBZ, $-k_{s} / 2 \leq k_{x}<k_{s} / 2$ and $-k_{s} \leq k_{y}<k_{s}$, and $\psi_{e, o}$ are the amplitudes on even and odd lattice sites respectively. The dispersion relation of the lowest band for $J / K=1$ is depicted in Fig. 5.9a. It exhibits a single minimum at $\mathbf{k}_{e}=$ $\left(-k_{s} / 4,-k_{s} / 4\right)$ and the corresponding ground state has an equal weight on even and odd sites, $\left|\psi_{e}\right|=\left|\psi_{o}\right|$. According to the intrinsic structure of the eigenstates we expect 


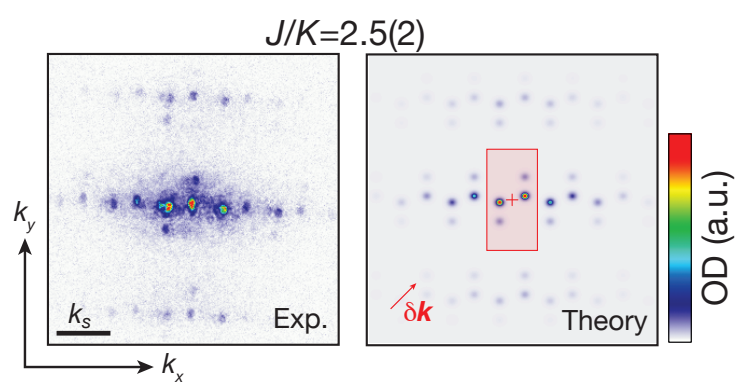

Figure 5.10: Momentum distribution after TOF for the staggered flux lattice with anisotropic coupling strength $J / K=2.5(2)$. The momentum distribution depicted in the left panel was measured after $t_{T O F}=20 \mathrm{~ms}$ using the sequence illustrated in Fig. 5.7 for $V_{y}=10.0(3) \mathrm{E}_{\mathrm{rs}}$. The theoretical momentum distribution (right panel) was calculated by exact diagonalization of a $31 \times 31$ lattice with harmonic confinement $\omega_{x} /(2 \pi)=\omega_{y} /(2 \pi)=20 \mathrm{~Hz}$. The red rectangle marks the dimensions of the FBZ and the cross marks its center position at zero momentum. The distribution exhibits four momentum components within the FBZ. The components that correspond to odd sites are shifted by $\delta \mathbf{k}=\left(k_{s} / 2, k_{s} / 2\right)$ relative to the momentum components of even sites. Each of the two images was normalized to its maximum intensity. (Data taken from Ref. [68])

to obtain a momentum component at $\mathbf{k}_{e}$ and an additional one at $\mathbf{k}_{o}=\mathbf{k}_{e}+\delta \mathbf{k}$ shifted by $\delta \mathbf{k}=\left(k_{s} / 2, k_{s} / 2\right)$ due to the additional phase factor corresponding to the wavefunction amplitude on odd sites (red crosses in Fig. 5.9a). In the measured TOF images additional momentum components appear at multiples of the reciprocal magnetic lattice vectors $\left(k_{s}, 0\right)$ and $\left(0,2 k_{s}\right)$ as shown in Fig. $5.8 \mathrm{~b}$, in agreement with theory.

The spatial distribution of the ground-state wave function is illustrated in Fig. 5.9b. The artificial gauge field causes the phase distribution of the atomic wave function to be non-uniform across the lattice. The value of the phase on each site is encoded in the direction of the black arrows in the figure. In order to make the appearance of phase vortices more clear these values were further interpolated for positions between neighboring lattice sites (background color in Fig. 5.9b). There is one vortex whenever there is a phase-winding of $2 \pi$, as depicted in the legend of Fig. $5.9 \mathrm{~b}$. The winding can be clockwise or anti-clockwise (white arrows), which is referred to as the chirality of the vortex. For our value of the flux $\alpha=1 / 4$, there is one vortex every four plaquettes if one follows one row along $y$ in the lattice and the chirality of the vortex alternates between neighboring rows along $x$, which coincides with the direction where the flux is alternating in sign. The atomic density of the ground state is uniform (length of the arrows in Fig. 5.9b), which is consistent with equal amplitudes on even and odd sites $\left|\psi_{e}\right|=\left|\psi_{o}\right|$. 

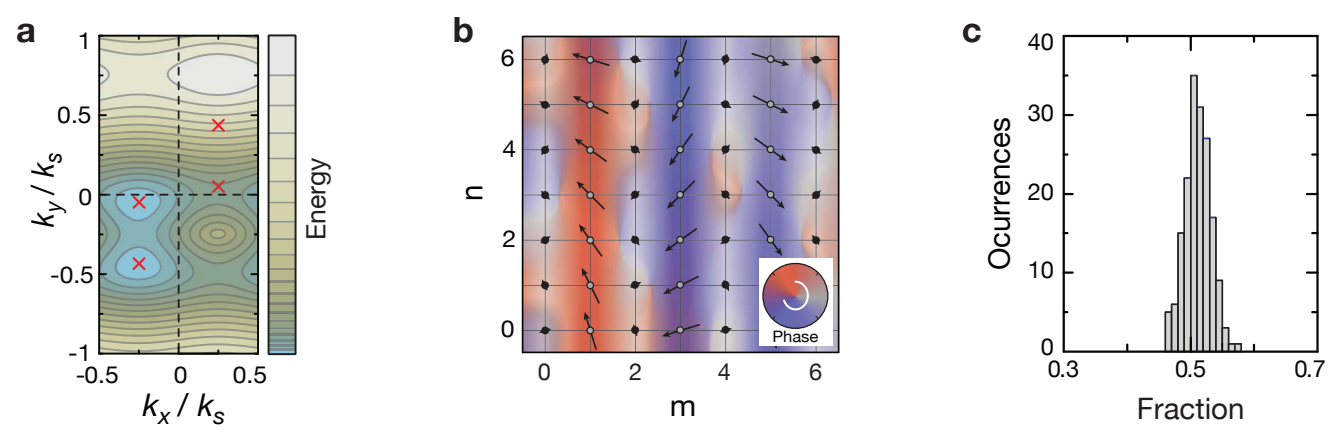

Figure 5.11: Ground-state properties for an anisotropic coupling strength $J / K=2.5(2)$. a The dispersion relation of the lowest band features two degenerate minima at $\mathbf{k}_{L}^{e}$ (lower momentum state) and $\mathbf{k}_{U}^{e}$ (upper momentum state). This leads to four momentum components within the FBZ (red crosses), where the two additional ones are located at $\mathbf{k}_{i}^{o}=\mathbf{k}_{i}^{e}+\delta \mathbf{k}, i=\{L, U\} . \mathbf{b}$ Spatial distribution of the phase (direction of the arrows) and amplitude (length of the arrows) for one of the two ground states $\Psi_{L}$. The other ground state $\Psi_{U}$ shows a similar distribution shifted by one lattice constant. The background color represents the values of the phase which were interpolated between neighboring lattice sites. c Measured atom fraction in the momentum components corresponding to the ground state $\Psi_{L}$. The histogram was obtained from 172 identical measurements. (Data taken from Ref. [68])

\subsubsection{Dependence on the coupling ratio $J / K$}

To study the ground state for different coupling ratios $J / K$ the depth of the lattice along $y$ was varied between $9.5 \mathrm{E}_{\mathrm{rs}}$ and $16.5 \mathrm{E}_{\mathrm{rs}}$, which corresponds to the range $0.6 \leq J / K \leq$ 2.8. The bifurcation point where the single minimum splits into two degenerate ones is expected to occur at $(J / K)_{c}=\sqrt{2}$ (Sect. 5.1). We start by studying the ground state for $J / K=2.5(2)$ above the critical point using the same sequence as illustrated in Fig. 5.7. The only difference was that the $y$-lattice was ramped to $V_{y}=10.0(3) \mathrm{E}_{\mathrm{rs}}$ during the first ramp. This corresponds to the bare tunnel coupling $J_{y} / h=75(3) \mathrm{Hz}$. After the first ramp the $y$-lattice depth was kept constant until it was switched off for TOF imaging.

As displayed in the left panel of Fig. 5.10 we observed four momentum components within the first magnetic Brillouin zone in agreement with an exact diagonalization study as depicted in the right panel of the same figure. Due to the two-fold degeneracy of the ground state $\Psi_{i}, i=\{L, U\}$, we obtain twice as many momentum components compared to the situation of isotropic coupling $J / K=1$ studied in the previous section. Here, the index $L$ denotes the state with lower momentum components and $U$ the one with the upper components (Fig. 5.11a). Each of these two states $\Psi_{i}$ features two momentum components $\mathbf{k}_{i}^{e}$ and $\mathbf{k}_{i}^{o}=\mathbf{k}_{i}^{e}+\delta \mathbf{k}, \delta \mathbf{k}=\left(k_{s} / 2, k_{s} / 2\right)$, associated with even and odd lattice sites according to Eq. (5.38). For $J / K=2.5$, the dispersion relation of the lowest band displays one minimum at $\mathbf{k}_{L}^{e} \simeq\left(-0.25 k_{s},-0.45 k_{s}\right)$ and a second one at $\mathbf{k}_{U}^{e} \simeq\left(-0.25 k_{s},-0.052 k_{s}\right)$ as shown in Fig. 5.11a. The two momenta are split around $-k_{s} / 4$ by $\Delta k_{y} \simeq 0.40 k_{s}$. The relative weight between the components $\mathbf{k}_{i}^{e}$ and $\mathbf{k}_{i}^{o}$ deter- 


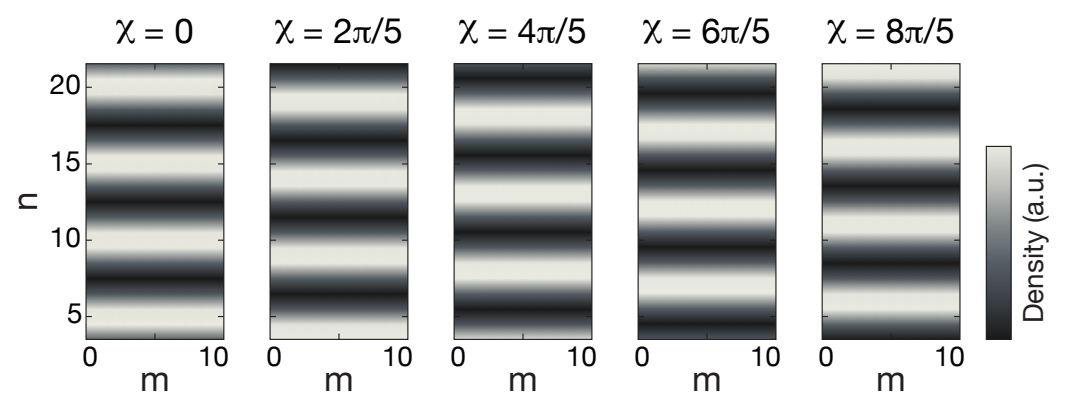

Figure 5.12: Spatial density of the ground-state wave function defined in Eq. 5.40 for $J / K=$ 2.5. For weakly interacting systems the ground state can be written as a superposition of the two degenerate states $\Psi_{L}$ and $\Psi_{U}$. The relative phase $\chi$ between them translates the density wave along $y$. For $J / K=2.5$ the density wave has a periodicity of five lattice constants. The distribution was calculated using the eigenvalue equation given in Eq. (5.39).

mines the spatial distribution of the ground-state wave function. In Fig. 5.11b we show the local density for one of the two ground states $\Psi_{L}$ (length of the arrows). It exhibits a charge density wave with larger amplitudes on odd sites. Using the eigenvalue equation (5.39) we computed the relative weight between even and odd sites $\left|\psi_{L}^{o}\right|^{2} /\left|\psi_{L}^{e}\right|^{2} \simeq 6.1$. Consequently, the momentum component at $\mathbf{k}_{L}^{o}$ (odd sites) is more intense than the one at $\mathbf{k}_{L}^{e}$ (even sites). For the state $\Psi_{U}$ the situation is reversed $\left|\psi_{U}^{o}\right|^{2} /\left|\psi_{U}^{e}\right|^{2} \simeq 1 / 6.1$ and the state features a charge density wave with larger population on even sites. Hence, the component $\mathbf{k}_{U}^{e}$ (even sites) has a higher intensity compared to the $\mathbf{k}_{L}^{o}$-component (odd sites). This is in qualitative agreement with the data shown in the left panel of Fig. 5.10. The spatial distribution of one of the two degenerate ground states $\Psi_{L}$ is illustrated in Fig. 5.11b. It shows that the phase of the wave function tends to align along the direction of larger coupling strength $J$, thereby frustrating the phase relation imprinted by the modulation. As a result the density is suppressed on every second stripe along $x$. The distribution for $\Psi_{U}$ exhibits a similar behavior but shifted by one lattice constant.

We further measured the relative population of the two ground states as depicted in Fig. 5.11c. Contrary to the triangular lattice with frustrated hopping studied in Ref. [67], where spontaneous symmetry breaking was observed, we do not see strong fluctuations in the measured atom fraction but observe an equal population in both states. Since there is a two-fold degeneracy in the system, the question arises if the atoms condense into the same single-particle state or if the ground state is fragmented [180]. In the presence of weak repulsive interactions it has been shown that fragmentation is in general unfavorable [181]. Following this result, it has been shown in Ref. [171], that the condensate ground state can be written in the following form

$$
\Psi_{c}=\frac{1}{\sqrt{2}}\left(\Psi_{L}+\mathrm{e}^{i \chi} \Psi_{U}\right)
$$

This state breaks the translation invariance along $y$. For $J / K=2.5$ the difference be- 
a

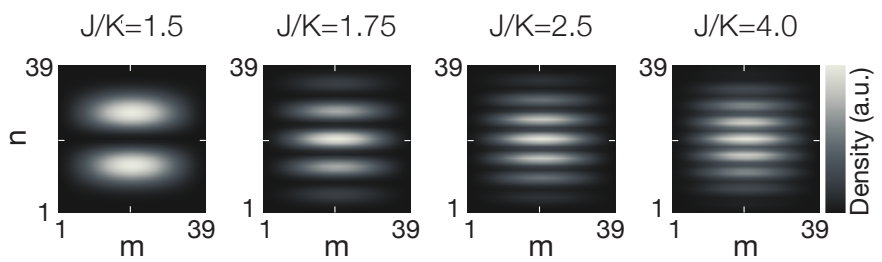

b

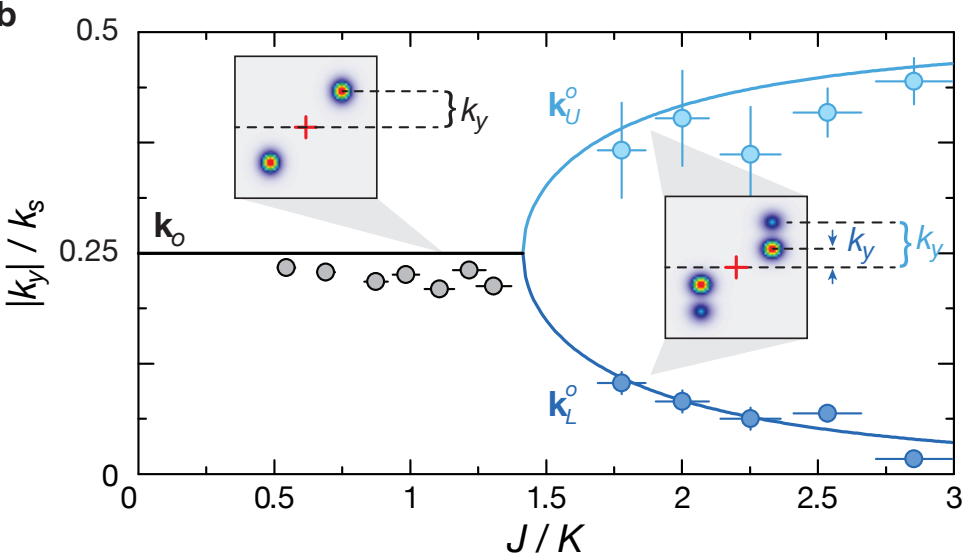

Figure 5.13: Density distribution and splitting of the momentum components along $y$ as a function of $J / K$. a The density distribution of the ground state was obtained by exact diagonalization of a finite lattice of dimension $39 \times 39$. The periodicity of the density wave increases with $\Delta k_{y}$ and approaches a maximum value of four lattice constants in the limit $J \gg K$ where the splitting approaches the value $\Delta k_{y}=k_{s} / 2$. Each image was normalized to to the maximum density. $\mathbf{b}$ Measured ground-state momentum components $k_{y}$ as a function of $J / K$ associated with odd lattice sites. For $J / K<\sqrt{2}$ we observe a single momentum component at $k_{y}=k_{s} / 4$. Above that value the momentum components split around this position. In the limit $J \gg K$ the two momentum components tend to $k_{y}=0$ and $k_{y}=k_{s} / 2$. The solid lines are calculated using the two-dimensional eigenvalue Eq. (5.39) in agreement with our data. The horizontal error bars depict the experimental uncertainty in the coupling ratio $J / K$ and the vertical error bars display the fitting error in the determination of the peak positions. The insets show the calculated momentum distribution in the FBZ and the red cross marks the position of zero momentum. (Data shown in (b) taken from Ref. [68])

tween the two momentum components $\mathbf{k}_{L}$ and $\mathbf{k}_{U}$ along $y$ is $\Delta k_{y} \simeq 0.40 k_{s}$. As a result the state $\Psi_{c}$ features a density wave, which is commensurate with the lattice spacing and exhibits a periodicity of five lattice constants (Fig. 5.12). For weak interactions compared to the kinetic energy, it was found that the system possesses an infinite degeneracy in $\chi$, because the interaction energy does not depend on this phase [171]. For stronger interactions this degeneracy is reduced and the density "locks" to the lattice sites and the remaining degree of degeneracy is determined by the periodicity of the density pattern in units of the lattice constant [171]. For $J / K=2.5$ this would correspond to a five-fold degeneracy. Intuitively this can be seen by looking at the action of $\chi$, which corresponds to a translation of the density distribution along $y$ (Fig. 5.12). 
The splitting of the momentum components along $y$ increases monotonically with $J / K$ for values larger than the critical ratio $(J / K)_{c}=\sqrt{2}$. For very large couplings $J \gg K$ it approaches a maximum splitting of $\Delta k_{y}=k_{s} / 2$, which corresponds to a density wave of the ground state in the lattice with a period of four lattice constants (Fig. 5.13a).

We measured the projection of the momentum components on the $y$-axis as a function of $J / K$ using the same experimental sequence as described in the previous section. The depth of the $y$-lattice was set during the first ramp to its final value, while the effective coupling along $x$ remained unchanged $K=30(2) \mathrm{Hz}$. As shown in Fig. 5.13b the measured momenta are in quantitative agreement with analytical calculations using the eigenvalue equation (5.39). The small panels above the data depict the density of the ground state on a finite lattice, which was computed with exact diagonalization. These results are in agreement with the previous discussion based on weak interactions. The period of the density wave decreases with the splitting of momentum components $\Delta k_{y}$ along $y$.

For certain values $\Delta k_{y}$ the corresponding periodicity of the density wave is incommensurate with the lattice spacing. It was demonstrated that in this regime an incommensuratecommensurate phase transition is expected to occur with increasing interactions, where above a critical value of the interaction, the periodicity of the density wave locks to the one of the underlying lattice [171].

\subsubsection{Micro-motion in the staggered flux lattice}

So far we were discussing the experimental results in terms of the Floquet Hamiltonian (5.37) which was derived in the rotating frame. We know, however, that the micromotion potentially influences TOF experiments. For large driving frequencies compared to the bare tunnel coupling the micro-motion essentially corresponds to the transformation from the lab frame to the rotating frame and is determined by Eqs. (5.35) and (5.36). This leads to the appearance of additional momentum components whose amplitudes scale with the ratio $V_{0} /(\hbar \omega)$. For very small driving amplitudes $V_{0} /(\hbar \omega) \ll 1$ we do not expect to observe additional momentum components other than the ones discussed in the previous section (see also Sect. 5.4). For our experimental parameters $V_{0} /(\hbar \omega) \simeq 0.48$, however, the micro-motion might play a role. In the experiments described above the modulation was switched on abruptly and after a waiting time of $10 \mathrm{~ms}$ (44 driving periods) coherence was restored. The relaxation process after switching on the periodic modulation which led to a restored phase coherence is not yet well understood. However, the data can be compared with the full-time evolution under the assumption that the system did relax to the ground state $\Psi_{\text {rot }}$ of the Floquet Hamiltonian (5.37) after the coherence was restored. The measured momentum components depicted in Fig. 5.8b, 5.10 and 5.13b support this assumption. In this case the corresponding state in the lab frame is determined by 


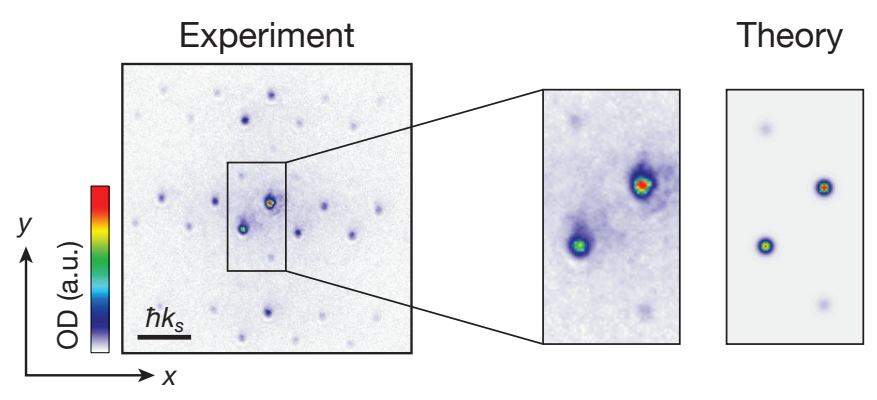

Figure 5.14: Comparison of the measured momentum distribution with theoretical calculations including the micro-motion for $J / K=1$. The panel on the left shows the measured momentum distribution (same data as in Fig. 5.8b) which exhibits two prominent momentum components in the FBZ (black frame). The panel in the middle focuses on the structure within the FBZ revealing two additional weaker momentum components, which are in agreement with theoretical calculations based on Eq. (5.41) for $V_{0} /(\hbar \omega) \simeq 0.48$.

$$
\begin{aligned}
\Psi_{\text {lab }}(T) & =\exp \left[i \hat{\mathcal{M}}_{\text {stagg }}^{+}(T)\right] \Psi_{\text {rot }}, \\
\hat{\mathcal{M}}_{\text {stagg }}(T) & =\sum_{m, n}\left(-\frac{V_{0}}{\hbar \omega} \cos \left[\varphi_{m, n}\right]\right) \hat{n}_{m, n},
\end{aligned}
$$

with $\varphi_{m, n}=\frac{\pi}{2}(m-n+1)+\phi_{0}$ as derived in Sect. 5.3 and 5.4. Figure 5.14 depicts the calculated momentum distribution in the lab frame for isotropic coupling $J / K=1$ in comparison with the experimental data, which was already shown in Fig. 5.8b. We observe two weak additional momentum components in the FBZ compared to the distribution obtained in the rotating frame (right panel Fig. 5.8b), whose amplitude depends on $V_{0} /(\hbar \omega)$. They are located at $\left(-k_{s} / 4,3 k_{s} / 4\right)$ and $\left(k_{s} / 4,-3 k_{s} / 4\right)$ in agreement with our experimental data. Note that the position and weight of the additional peaks do not depend on the relative phase $\phi_{0}$ between the two running-wave beams, which sets the initial phase of the modulation (Sect. 5.3). The full-time dynamics within one Floquet period leads to small oscillations in the weight of the different momentum components but the positions remain constant.

\subsection{Local probe of the artificial gauge field}

The effect of the artificial gauge field can be probed locally by making use of the twodimensional superlattice potential (Sect. 4.3). This allows us to study the dynamics of single atoms on the level of four-site square plaquettes (Fig. 5.15a) and enables an experimental determination of the flux $\Phi$ per plaquette.

The setup consists of a tilted double-well potential along $x$ and a symmetric one along $y$. 
Tunneling between neighboring double wells is suppressed due to high potential barriers, so that all dynamics is restricted to the four sites of one plaquette. The sites are denoted by $\mathcal{R}=\{A, B, C, D\}$ as depicted in Fig. 5.15a. All observables measured in the experiment are averaged over a $3 \mathrm{D}$ array of individual plaquette realizations. The flux through each plaquette exhibits the same sign for all realizations because only every other plaquette participates in the measurement and all of them exhibit an energy offset between neighboring sites with the same sign.

\section{Experimental sequence}

The experimental sequence (Fig. 5.15b) started by loading a BEC into a 3D optical lattice in the Mott-insulating regime with at most two atoms per lattice site [44]. The potential was created using the two long lattices in the horizontal plane and the vertical lattice with depths $V_{x l}=V_{y l}=35(1) \mathrm{E}_{\mathrm{rl}}$ and $V_{z}=30(1) \mathrm{E}_{\mathrm{z}}$. To remove all atoms on doubly occupied sites a filtering sequence was applied as described in Sect. 4.5 and Ref. [34]. Subsequently the short lattice along $x$ was ramped up within $10 \mathrm{~ms}$ to $V_{x}=5.0(2) \mathrm{E}_{\mathrm{rs}}$ with $\varphi_{S L}^{x}=0.20(1) \mathrm{rad}$ in order to load the atoms into the ground state of the tilted double-well potential. This corresponds to an energy offset $\Delta / h=6.0(1) \mathrm{kHz}$, which was calibrated independently using spectroscopy measurements (Sect. 4.3.2). As a last step the short lattice along $y$ was switched on within $1 \mathrm{~ms}$ to $V_{y}=14(1) \mathrm{E}_{\mathrm{rs}}$ in order to create an initial state where each atom is delocalized over the left bond in the plaquette $\left|\psi_{1}\right\rangle=(|A\rangle+|D\rangle) / \sqrt{2}$. The corresponding bare tunneling amplitudes are $J_{x} / h=2.0(1) \mathrm{kHz}$ and $J_{y} / h=0.17(2) \mathrm{kHz}$. To initiate the dynamics the modulation was suddenly switched on. This induced resonant tunneling to the $B$ and $C$ sites for $\hbar \omega=\Delta$. The effective coupling strength $K / h=0.32(1) \mathrm{kHz}$ was calibrated independently as explained in Sect. 4.4. In the weak driving limit $V_{0} /(\hbar \omega) \ll 1$ the effective coupling along $y$ is approximately given by the bare coupling strength, $J \simeq J_{y}$. For our parameters $V_{0} /(\hbar \omega) \simeq 0.23$. The dynamics of a single atom in the plaquette can be described by the effective $4 \times 4$ Hamiltonian $\hat{H}_{P}$, written in the basis $\{|A\rangle,|B\rangle,|C\rangle,|D\rangle\}$

$$
\hat{H}_{P}=-\left(\begin{array}{cccc}
0 & K & 0 & J \\
K & 0 & J & 0 \\
0 & J & 0 & K \mathrm{e}^{\mp i \Phi} \\
J & 0 & K \mathrm{e}^{ \pm i \Phi} & 0
\end{array}\right),
$$

with $\Phi=\pi / 2$. The sign of the complex tunneling amplitude depends on the direction of the flux and can be controlled by changing the sign of the modulation frequency $\hbar \omega= \pm \Delta$.

\subsubsection{Phase evolution}

We made use of two different detection techniques to study the dynamics within the plaquettes, i.e. phase and density evolution. Using expansion imaging we can access 
a

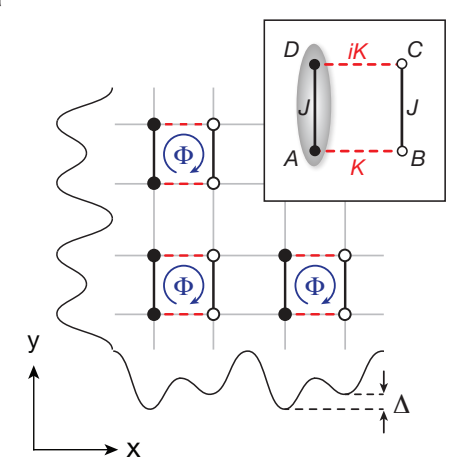

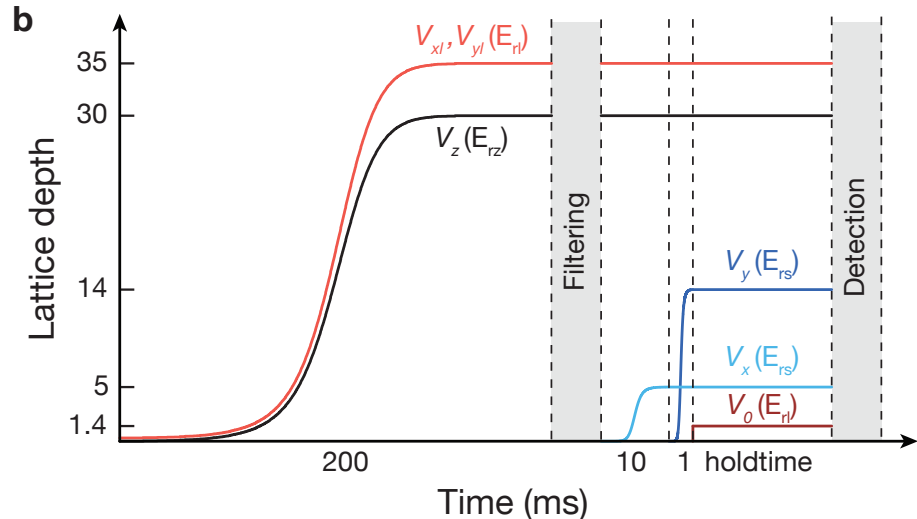

Figure 5.15: Schematic drawing of the setup and the experimental sequence. a Making use of two superlattice potentials along $x$ and $y$ the lattice is partitioned into four-site square plaquettes where tunneling between the plaquettes is suppressed by a high potential barrier. Tunneling along $x$ is further inhibited by the tilt $\Delta$ and later restored with the running-wave beams depicted in Fig. 5.4. The flux $\Phi$ exhibits the same sign for each plaquette potential that contributes to the signal. The dynamics within one plaquette can be described by the Hamiltonian in (5.42), where the sites are labeled as $\mathcal{R}=\{A, B, C, D\}$. The gray shaded area illustrates the initial state $\left|\psi_{1}\right\rangle=(|A\rangle+|D\rangle) / \sqrt{2}$. $\mathbf{b}$ Experimental sequence used to probe the artificial magnetic field in a local manner for the two detection methods described in the main text, i.e. phase evolution and real space evolution. The filtering sequence is used to remove double occupancies from the trap as described in Sect. 4.5.

information about the phase distribution of the single-particle state in the plaquette. The evolution can be rather complex but to get an intuitive understanding of it we consider the limit $J \ll K$, where the dynamics along $y$ is suppressed. As discussed above, we quench the system from the initially tilted plaquette without flux where tunneling is inhibited, to a symmetric one with flux $\Phi=\pi / 2$. In this configuration the initial state $\left|\psi_{1}\right\rangle=(|A\rangle+|D\rangle) / \sqrt{2}$ on the left bond couples to the one on the right bond $\left|\psi_{2}\right\rangle=$ $(|B\rangle+i|C\rangle) / \sqrt{2}$. Note that this state involves a phase difference between neighboring sites which is a manifestation of the flux through the plaquette. By suddenly switching off all fields this value can be evaluated from the interference pattern recorded after TOF. As an example we consider the single-particle state $|\psi\rangle=\left(|A\rangle+\mathrm{e}^{i \gamma}|D\rangle\right) / \sqrt{2}$, with arbitrary phase $\gamma$. The density distribution after TOF is a double-slit interference pattern along $y$ proportional to $\cos \left(k_{t} y+\gamma\right)$ times an envelope determined by the Wannier function, where $k_{t}=m_{a t} a /\left(\hbar t_{T O F}\right)[33,34]$. For the initial state $\left|\psi_{1}\right\rangle$ one expects a symmetric double-slit pattern as shown in Fig. 5.16a, while for the state $\left|\psi_{2}\right\rangle$ one would expect to observe a pattern that is shifted due to the relative phase between the $B$ and $C$ site.

For our experimental parameters $J / K \approx 0.5$ the evolution is expected to be more complex. To access information about the phase between the lower and the upper bond we 

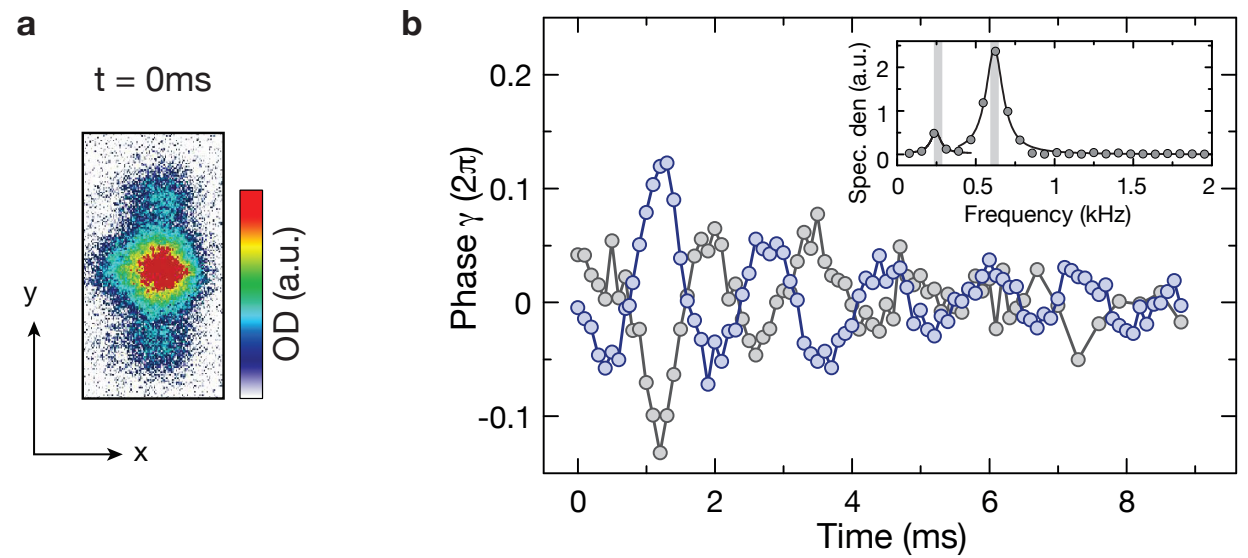

Figure 5.16: Phase evolution in isolated four-site square plaquettes. a Double-slit interference pattern for the initial state $\left|\psi_{1}\right\rangle=(|A\rangle+|D\rangle) / \sqrt{2}$ obtained after $t_{T O F}$. (Data taken from Ref. [69]) $b$ Phase evolution obtained from the double-slit patterns after integrating the density distributions along $x$ for $\hbar \omega=\Delta$ (blue) and $\hbar \omega=-\Delta$ (gray). The inset shows the Fourier transformation for $\hbar \omega=\Delta$ revealing two frequency components at $0.24(6) \mathrm{kHz}$ and $0.62(13) \mathrm{kHz}$ in agreement with theory (gray vertical lines). The theoretical evolution was obtained by solving the time-dependent Schrödinger equation associated with Hamiltonian (5.42) numerically. (Data taken from Ref. [68])

first integrated the momentum distribution along $x$ and then fitted the relative phase $\gamma$ of the double-slit interference pattern. The measured evolution $\gamma(t)$ is depicted in Fig. 5.16b. The blue data points show the evolution for $\hbar \omega=\Delta$ and the gray data points depict the evolution for $\hbar \omega=-\Delta$. The fact that the values of the phases differ only by a sign is a manifestation of the broken time-reversal symmetry in the system.

\subsubsection{Quantum cyclotron orbit}

Complementary to the measurement above is the observation of the density evolution in real space. The experimental sequence used for these measurements was the same as described above (Fig. 5.15). However, the final lattice parameters of the short lattices were changed in order to obtain different tunneling amplitudes: $V_{x}=7.0(2) \mathrm{E}_{\mathrm{rs}}$ and $V_{y}=10.0(2) \mathrm{E}_{\mathrm{rs}}$, corresponding to the coupling strengths $J_{x} / h=1.2(1) \mathrm{kHz}$ and $J_{y} / h=$ $0.50(2) \mathrm{kHz}$. The effective coupling $K / h=0.28(1) \mathrm{kHz}$ and the resonance condition $\Delta / h=4.9(1) \mathrm{kHz}$ were calibrated independently as described in Sect. 4.4. The effective coupling along $y$ is approximately given by $J \simeq J_{y}$ and the modulation amplitude was $V_{0} /(\hbar \omega) \approx 0.34$.

The sequence started by preparing single atoms per plaquette in the initial state $\left|\psi_{1}\right\rangle$, which is a superposition on $A$ and $D$ sites. The following real-space evolution after suddenly switching on the modulation was measured by performing a site-resolved detection as described in Sect. 4.6, which enabled us to determine the number of atoms 
a

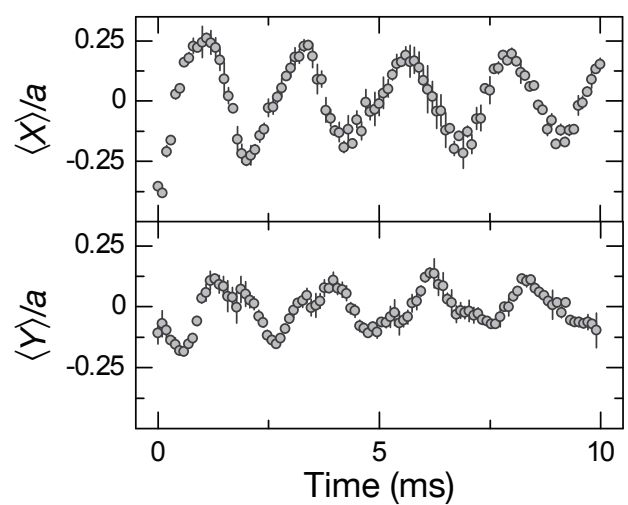

b

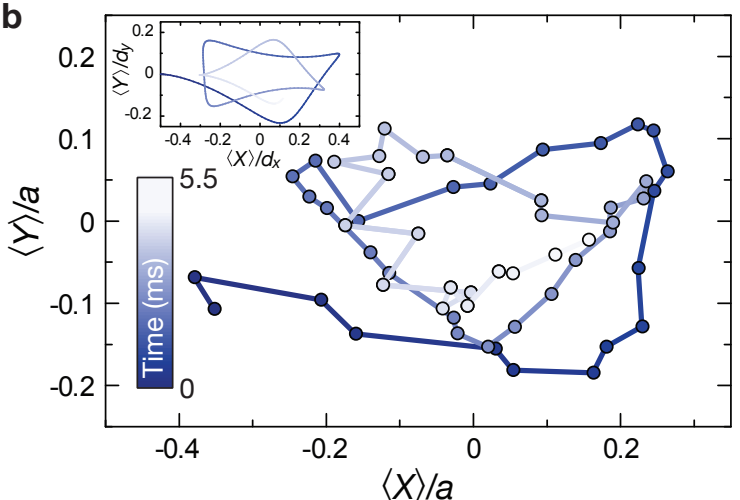

Figure 5.17: Real-space evolution of single atoms in isolated four-site square plaquettes. a Mean atom positions along $x$ and $y$ in the plaquette $\langle X\rangle / a$ and $\langle Y\rangle / a$. Each data point is an average over three measurements. The error bars depict the corresponding standard deviation. b Cyclotron orbit obtained from the mean atom positions. The inset shows the theoretical curve calculated by solving the time-dependent Schrödinger equation numerically for a flux $\Phi=$ $0.8 \times \pi / 2$ and a $1 / e$-damping time of $13 \mathrm{~ms}$. This value was obtained from damped sine fits to $\langle X\rangle / a$ and $\langle Y\rangle / a$ shown in (a). (Data taken from Ref. [68])

$N_{\mathcal{R}}$ on each site in the plaquette. Note that contrary to the site-resolved measurements with high-resolution detection techniques [50, 163], here, we measure the number of atoms on each site of the plaquette averaged over a 3D array of local copies of the same system. For the detection atoms located on different sites in the plaquette are transferred to higher Bloch bands and a subsequent band-mapping sequence allows for a determination of the corresponding band occupations. The mean positions along $x$ and $y$ in the plaquette are defined according to

$$
\begin{aligned}
& \langle X\rangle=\left(-N_{A}+N_{B}+N_{C}-N_{D}\right) \frac{a}{2 N}, \\
& \langle Y\rangle=\left(-N_{A}-N_{B}+N_{C}+N_{D}\right) \frac{a}{2 N},
\end{aligned}
$$

where $N$ is the total atom number (Fig. 5.17a). Initially all atoms are located on the left bond in the plaquette with $\langle X\rangle(t=0)=-0.5$ and $\langle Y\rangle(t=0)=0$. After switching on the modulation the atoms start to tunnel towards the right bond ( $B$ - and $C$-sites). Without magnetic field the atoms would simply undergo Rabi oscillations between the left and right bond, while no dynamics would occur along $y$, i.e. $\langle Y\rangle(t)=0$ for all times. Instead we observe that the particles are deflected along $y$, perpendicular to the initial direction of the motion, which is reminiscent of the Lorentz force acting on a charged particle in a magnetic field. The evolution follows a small-scale cyclotron orbit as depicted in Fig. 5.17b analog to the classical cyclotron orbit of a charged particle in a magnetic field. The theoretical evolution was calculated numerically with Hamiltonian (5.42), where we used the independently calibrated values of $J$ and $K$. Leaving the 
a

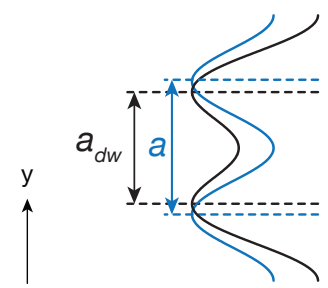

b

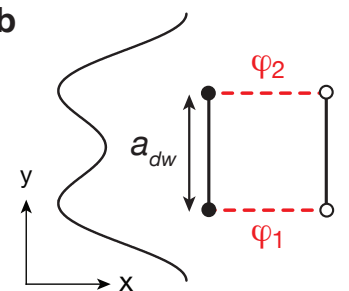

Figure 5.18: Reduced flux in isolated plaquette potentials. a Schematic drawing of the usual lattice potential with lattice constant $a$ (blue) and the double-well potential used to restrict the dynamics within two sites (black). The distance between the two wells is slightly smaller than the usual lattice constant $a_{\mathrm{dw}}<a$. b Complex phase factors imprinted by the modulation in the plaquettes. The reduced distance $a_{\mathrm{dw}}$ also reduces the flux per plaquette $\varphi_{1}-\varphi_{2}=\Phi a_{\mathrm{dw}} / a$.

flux $\Phi$ as a free fit parameter we obtained an experimental value for the flux $\Phi_{\exp }=$ $0.73(5) \times \pi / 2$. The deviation from $\Phi=\pi / 2$ for a homogeneous lattice is due to a reduced distance between lattice sites $a_{\mathrm{dw}}<a$ when partitioning the lattice into isolated four-site square plaquettes (Fig. 5.18). In this case the flux per plaquette is $\Phi a_{\mathrm{dw}} / a$. For our experimental parameters this corresponds to $a_{\mathrm{dw}} / a \approx 0.78(1)$, which qualitatively explains the reduced value of the flux. Residual deviations might be due to an angle mismatch between the running-wave beams and the lattice axes.

\subsection{Full-time dynamics of the cyclotron orbits}

In the previous section we discussed the cyclotron orbits based on the effective Floquet Hamiltonian in the rotating frame given in Eq. (5.42). This Hamiltonian was derived in the high-frequency limit $\hbar \omega \simeq \Delta \gg J_{x}$. For our experimental parameters $\Delta / J_{x} \simeq$ 3.56 , however, this assumption is not well fulfilled and corrections on the order of $J_{x} / \Delta$ (Sect. 3.1.4) might have to be taken into account. In addition the evolution depicted in Fig. 5.17 was measured non-stroboscopically, which is only valid in the high-frequency limit (Sect. 3.1.4), and the initial phase of the driving was neglected. In this section we are going to present a detailed discussion of the full-time dynamics, thereby verifying the description in terms of the simple effective Hamiltonian given in Eq. (5.42).

The complete time-dependent Hamiltonian without any approximations written in the basis $\{|A\rangle,|B\rangle,|C\rangle,|D\rangle\}$ is given by

$$
\hat{H}(t)=-\left(\begin{array}{cccc}
\Delta / 2 & J_{x} & 0 & J_{y} \\
J_{x} & -\Delta / 2 & J_{y} & 0 \\
0 & J_{y} & -\Delta / 2 & J_{x} \\
J_{y} & 0 & J_{x} & \Delta / 2
\end{array}\right)+\left(\begin{array}{cccc}
\Delta_{A}(t) & 0 & 0 & 0 \\
0 & \Delta_{B}(t) & 0 & 0 \\
0 & 0 & \Delta_{C}(t) & 0 \\
0 & 0 & 0 & \Delta_{D}(t)
\end{array}\right)
$$




$$
\begin{aligned}
\Delta_{A}(t) & =V_{0} \cos \left(\omega t+\phi_{0}\right), & \Delta_{B}(t) & =V_{0} \cos \left(\omega t+\phi_{0}+\pi / 2\right), \\
\Delta_{C}(t) & =V_{0} \cos \left(\omega t+\phi_{0}+\pi\right), & \Delta_{D}(t) & =V_{0} \cos \left(\omega t+\phi_{0}+\pi / 2\right) .
\end{aligned}
$$

All numerical calculations presented in this section are obtained by solving the timedependent Schrödinger equation associated with this Hamiltonian.

\subsubsection{Off-resonant driving}

We have seen in Sect. 3.1.4 that for arbitrarily large energy offsets $\Delta \gg J_{x}$ periodic modulation at the approximate resonance frequency $\hbar \omega=\Delta$ leads to the desired effective Floquet Hamiltonian. However, if $\Delta$ is on the order of the tunnel coupling $J_{x}$, additional detuning terms in the effective Hamiltonian as given in Eq. (3.53) will become important. This term causes a residual energy offset between neighboring sites $J_{x}^{2} /(\hbar \omega)$ along the $x$-direction in the plaquette, which suppresses tunneling between the left and right side. In Figure 5.19a we show the numerical evolution for our experimental parameters if the periodic modulation is performed with the approximate resonance frequency $\hbar \omega=\Delta$. As in the experiment we start the evolution with an initial state that is delocalized on the left bond in the plaquette $\left|\psi_{1}\right\rangle=(|A\rangle+|D\rangle) / \sqrt{2}$, see Fig. 5.15a. However, after switching on the modulation with the approximate resonance frequency atoms cannot tunnel resonantly to the $B$ and $C$ sites due to the additional detuning term. This leads to off-resonant Rabi oscillations between the left and right bond with reduced amplitude and increased frequency (lower panels in Fig. 5.19a). The squeezing of the off-resonant cyclotron orbit is dramatic even though the detuning is only on the order of $0.1 \Delta$. Similar effects were observed in Ref. [122] and with interacting particles [182].

The exact resonance condition

$$
\hbar \omega_{\text {res }}=\sqrt{\Delta^{2}+4 J_{x}^{2}}
$$

is determined by the energy difference between the two eigenstates of the static Hamiltonian as defined in Sect. 3.1.4, Eq. (3.44). In our experiments the resonance condition is calibrated through spectroscopy measurements (Sect. 4.3.2), hence, the resonance frequency is automatically set to the exact one as defined in Eq. (5.46).

Let us consider periodic modulation of the double-well along $x$ with the exact resonance condition. The dynamics can be described by the following two-level Hamiltonian

$$
\begin{gathered}
\hat{H}(t)=-J_{x}(|0\rangle\langle 1|+| 1\rangle\langle 0|)+\Delta \hat{P}^{1}+V_{0} \cos \left(\omega_{\text {res }} t+\varphi\right) \hat{P}^{0}, \\
\hat{P}^{\alpha}=|\alpha\rangle\langle\alpha|, \quad \alpha=\{0,1\},
\end{gathered}
$$

where $\hbar \omega_{\text {res }}=\sqrt{\Delta^{2}+4 J_{x}^{2}} \simeq \Delta+2 J_{x}^{2} / \Delta$ and the two levels correspond to the two sites of the double-well. After performing a unitary transformation according to Eqs. (3.27)(3.29) with 

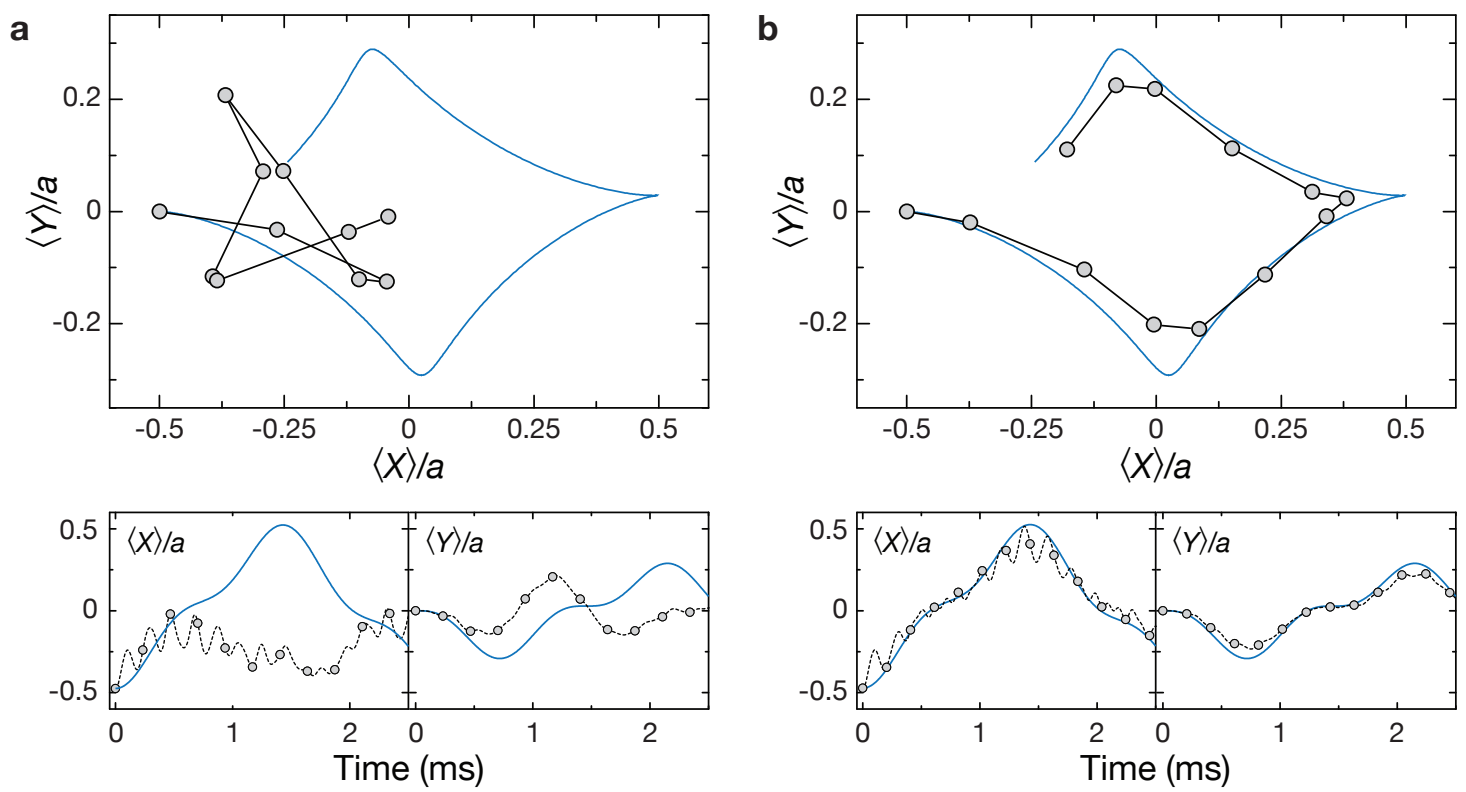

Figure 5.19: Calculated cyclotron orbits for off-resonant and resonant modulation in the plaquettes. The numerical evolutions (black) were obtained with the exact time-dependent Hamiltonian defined in Eq. (5.45). The corresponding full-time evolution is depicted with the dashed line in the lower panels and the dots highlight the dynamics at stroboscopic times. The evolution obtained using the effective Floquet Hamiltonian in Eq. (5.42) is shown in blue. a Periodic driving at the approximate resonance condition $\hbar \omega \simeq \Delta=4.3 \mathrm{kHz} \times h$ causes a dramatic shrinking of the cyclotron orbit (black) for our experimental parameters $\Delta / J_{x}=3.56$ compared to the evolution governed by the effective Hamiltonian (blue). $\mathbf{b}$ Numerical evolution of the cyclotron orbit for the exact resonance condition $\hbar \omega_{\text {res }}=4.9 \mathrm{kHz} \times h$ (black) as defined in Eq. (5.46). Contrary to (a) the amplitude of the orbit is comparable to the one obtained using the effective Floquet Hamiltonian (blue). The numerical results shown in black were obtained by averaging over the different individual plaquettes realized in the experiment, which will be discussed in Sect. 5.7.2. All evolutions were computed for the experimental parameters $K / h=0.28 \mathrm{kHz}, J / h=0.47 \mathrm{kHz}$ and $t \leq 2.5 \mathrm{~ms}$, which correspond to the measurements depicted in Fig. 5.17.

$$
\hat{R}(t)=\exp \left[i \omega_{\text {res }} t \hat{P}^{1}\right]
$$

the transformed Hamiltonian takes the following form

$$
\begin{aligned}
\hat{\mathcal{H}}(t) & =\hat{\mathcal{H}}^{(0)}+\hat{\mathcal{H}}^{(+1)} \mathrm{e}^{i \omega_{\text {res }} t}+\hat{\mathcal{H}}^{(-1)} \mathrm{e}^{-i \omega_{\text {res }} t}, \\
\hat{\mathcal{H}}^{(0)} & =-\frac{2 J_{x}^{2}}{\Delta} \hat{P}^{1}, \\
\hat{\mathcal{H}}^{(+1)} & =-J_{x}|1\rangle\langle 0|+\frac{V_{0}}{2} \hat{P}^{0} \mathrm{e}^{i \varphi}, \\
\hat{\mathcal{H}}^{(-1)} & =-J_{x}|0\rangle\langle 1|+\frac{V_{0}}{2} \hat{P}^{0} \mathrm{e}^{-i \varphi} .
\end{aligned}
$$


It contains an additional static term $\hat{\mathcal{H}}^{(0)}$ which tends to zero in the limit $J_{x} / \Delta \rightarrow 0$. The effective Floquet Hamiltonian can be derived according to Eq. (3.31)

$$
\begin{aligned}
\hat{\mathcal{H}}_{F} & =\hat{\mathcal{H}}^{(0)}+\frac{1}{\hbar \omega_{\text {res }}}\left[\hat{\mathcal{H}}^{(+1)}, \hat{\mathcal{H}}^{(-1)}\right] \\
& =-\frac{2 J_{x}^{2}}{\Delta} \hat{P}^{1}-\underbrace{\frac{J_{x}^{2}}{\hbar \omega_{\text {res }}}}_{\approx J_{x}^{2} / \Delta}\left(\hat{P}^{0}-\hat{P}^{1}\right)-\frac{J_{x} V_{0}}{2 \hbar \omega_{\text {res }}}\left(|0\rangle\left\langle 1\left|\mathrm{e}^{i \varphi}+\right| 1\right\rangle\langle 0| \mathrm{e}^{-i \varphi}\right) \\
& =\underbrace{-\frac{J_{x}^{2}}{\Delta}\left(\hat{P}^{0}+\hat{P}^{1}\right)}_{\text {global offset }}-\frac{J_{x} V_{0}}{2 \hbar \omega_{\text {res }}}\left(|0\rangle\left\langle 1\left|\mathrm{e}^{i \varphi}+\right| 1\right\rangle\langle 0| \mathrm{e}^{-i \varphi}\right) \\
& =-\frac{J_{x} V_{0}}{2 \hbar \omega_{\text {res }}}\left(|0\rangle\left\langle 1\left|\mathrm{e}^{i \varphi}+\right| 1\right\rangle\langle 0| \mathrm{e}^{-i \varphi}\right)+\text { cst. }
\end{aligned}
$$

This precisely corresponds to the Hamiltonian (5.42), which was considered in the discussion of the experimental data in the previous section, because the two detuning terms compensate each other. This is in agreement with the numerical simulations depicted in Fig. 5.19b, where the exact time-evolution governed by Hamiltonian (5.45) is compared with the one predicted by the effective Floquet Hamiltonian (5.42). The evolutions were calculated for our experimental parameters using the exact resonance frequency, which was obtained experimentally through spectroscopy measurements.

\subsubsection{Initial kick-operator and micro-motion}

The cyclotron orbits were measured experimentally at non-stroboscopic times during the evolution. It is not obvious that the experimental results obtained in this way simply match the evolution predicted by the effective Floquet Hamiltonian (5.42). In this section we discuss the complete full-time evolution determined by Eq. (3.33)

$$
\hat{U}_{P}(t)=\mathrm{e}^{-i \hat{\mathcal{M}}_{P}(t)} \mathrm{e}^{-\frac{i}{\hbar} \hat{H}_{P} t} \mathrm{e}^{i \hat{\mathcal{M}}_{P}(0)},
$$

where in the high-frequency limit $\hbar \omega \gg J_{x}, J_{y}$ the micro-motion operator is given by

$$
\hat{\mathcal{M}}_{P}(t)=\sum_{\mathcal{R}}\left[\frac{V_{0}}{\hbar \omega} \sin \left(\omega t+\varphi_{\mathcal{R}}\right)+\frac{\Delta_{\mathcal{R}}}{\hbar} t\right] \hat{n}_{\mathcal{R}},
$$

and $\hat{H}_{P}$ is the effective plaquette Hamiltonian defined in Eq. (5.42). Here $\varphi_{\mathcal{R}}$ denotes the phase of the driving on site $\mathcal{R}, \Delta_{\mathcal{R}}$ is the energy offset on each site and $\hat{n}_{\mathcal{R}}$ is the corresponding number operator. The phases $\varphi_{\mathcal{R}}$ and energy offsets $\Delta_{\mathcal{R}}$ are determined by Eq. (5.45).

There are two separate effects related to the full-time evolution which are discussed in this section. The first one is due to the initial phase of the driving. This effect is governed by the initial kick at $t=0$ described by $\hat{\mathcal{M}}_{P}(0)$. The second one concerns the micromotion within one Floquet period according to $\hat{\mathcal{M}}_{P}(t)$, which may lead to additional 

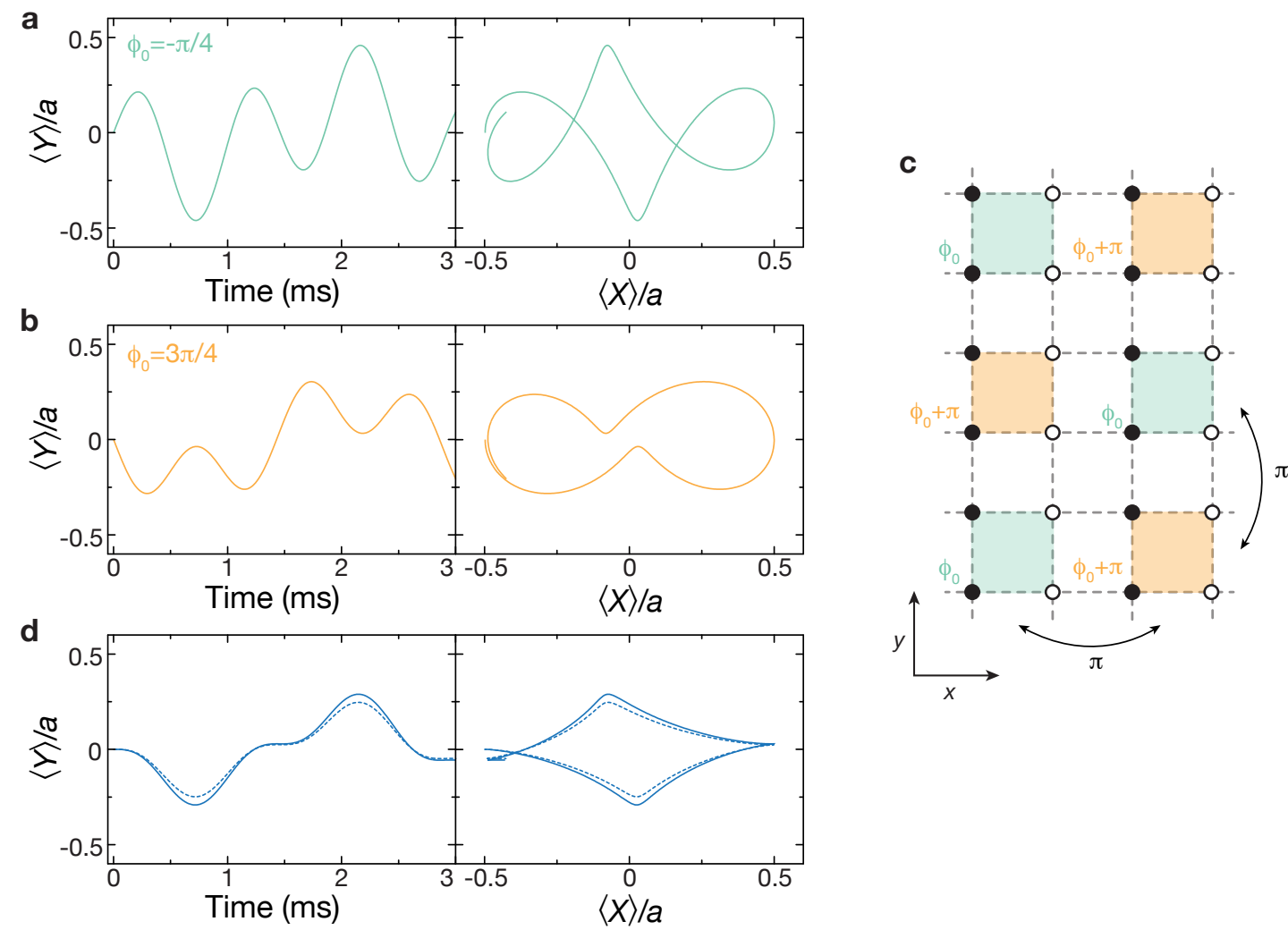

Figure 5.20: Impact of the initial kick introduced by the operator $\hat{\mathcal{M}}_{P}(0)$ on the mean atom position $\langle Y\rangle / a$ and the corresponding cyclotron orbit. The numerical evolutions were calculated according to the effective Hamiltonian (5.42) for an initial state $\left|\tilde{\psi}_{1}\right\rangle$ as defined in Eq. (5.56) and two different values of the initial phase: $\mathbf{a} \phi_{0}=-\pi / 4$ and $\mathbf{b} \phi_{0}=3 \pi / 4$. c Schematic drawing of the $2 \mathrm{D}$ array of isolated plaquettes. The phase of the on-site modulation $\varphi_{\mathcal{R}}$ is shifted by $\pi$ between adjacent plaquettes. In this figure the phase on the lower left corner of each plaquette is shown. $\mathbf{d}$ The dashed line depicts the average evolution of (a) and (b) and the solid line the one governed by the effective Hamiltonian (5.42) using the initial state $\left|\psi_{1}\right\rangle=(|A\rangle+|D\rangle) / \sqrt{2}$, which is the initial state prepared in the experiment. The parameters for the numerical simulations were chosen to match the ones for the measurements depicted in Fig. 5.17: $V_{0} /(\hbar \omega) \simeq V_{0} / \Delta=0.39$, $K / h=0.28 \mathrm{kHz}, J / h=0.47 \mathrm{kHz}$ and $t \leq 3 \mathrm{~ms}$.

oscillations of the experimental observables. In the high-frequency limit the operator $\hat{\mathcal{M}}_{P}(t)$ is proportional to the number operator $\hat{n}_{\mathcal{R}}$. Hence, it does not affect the shape of the cyclotron-orbits since we are measuring on-site densities and the micro-motion operator commutes with the number operator $\hat{n}_{\mathcal{R}}$.

\section{Initial kick introduced by the modulation}

In the high-frequency limit the density evolution can only deviate from the one predicted by the effective Floquet Hamiltonian (5.42) due to the initial kick at $t=0$ which 
is governed by the operator

$$
\mathcal{M}_{P}(0)=\sum_{\mathcal{R}} \frac{V_{0}}{\hbar \omega} \sin \left(\varphi_{\mathcal{R}}\right) \hat{n}_{\mathcal{R}}
$$

Note that strictly speaking the Floquet Hamiltonian (5.42) depends on the initial phase of the driving $\phi_{0}$. This term, however, has no impact on the dynamics as was verified numerically. For the sake of simplicity we have omitted related terms in the following discussion.

Acting with the operator $\mathcal{M}_{P}(0)$ on the initial state $\left|\psi_{1}\right\rangle=(|A\rangle+|D\rangle) / \sqrt{2}$ illustrated in Fig. 5.15 leads to the transformed initial state

$$
\left|\tilde{\psi}_{1}\right\rangle=\frac{1}{\sqrt{2}}\left(\exp \left[i \frac{V_{0}}{\hbar \omega} \sin \left(\varphi_{A}\right)\right]|A\rangle+\exp \left[i \frac{V_{0}}{\hbar \omega} \sin \left(\varphi_{D}\right)\right]|D\rangle\right),
$$

with $\varphi_{A}=\phi_{0}$ and $\varphi_{D}=\phi_{0}+\pi / 2$. This transformed initial state causes a modified evolution of the mean atom position $\langle Y\rangle / a$ along the $y$-direction, while the evolution along $x$ remains unaffected. In Figure $5.20 \mathrm{a}, \mathrm{b}$ the mean atom position $\langle Y\rangle / a$ and the corresponding cyclotron orbit are displayed for two different values of the initial phase $\phi_{0}=-\pi / 4$ (Fig. 5.20a) and $\phi_{0}=3 \pi / 4$ (Fig. 5.20b). The evolution was calculated using $\left|\tilde{\psi}_{1}\right\rangle$ as an initial state and evolving it numerically with the effective Floquet Hamiltonian (5.42) for $\Phi=\pi / 2$,

$$
\left|\tilde{\psi}_{P}\right\rangle(t)=\mathrm{e}^{-\frac{i}{\hbar} \hat{H}_{P} t}\left|\tilde{\psi}_{1}\right\rangle, \quad\left|\tilde{\psi}_{1}\right\rangle=\mathrm{e}^{i \hat{\mathcal{M}}_{P}(0)}\left|\psi_{1}\right\rangle .
$$

The initial phase $\phi_{0}$ has a large influence on the shape of the cyclotron orbit and is not actively stabilized in the experiment. As a result we should not be able to observe any kind of orbit in the experiment. However, as mentioned above the measured quantities are averaged over the 3D array of individual plaquette realizations. The configuration in the 2D plane is depicted in Fig. 5.20c, which reveals that there are only two different kinds of plaquettes. The phase of the on-site modulation is either $\varphi_{\mathcal{R}}$ or $\varphi_{\mathcal{R}}+\pi$. Averaging the numerical evolution over these two plaquette realizations leads to an evolution that matches almost exactly the one governed by the effective Floquet Hamiltonian using an initial state $\left|\psi_{1}\right\rangle$ (Fig. 5.20d). We verified that this is the case for any value of $\phi_{0}$.

\section{Micro-motion}

What remains to be discussed are finite frequency corrections to the micro-motion determined by $\hat{\mathcal{M}}_{P}(t)$. We assume that the system is modulated with the exact resonance frequency $\omega_{\text {res }}$ as defined in Eq. (5.46), which is the case for the measurements discussed in the previous section. As mentioned above, in the high frequency limit $J_{x} /\left(\hbar \omega_{\text {res }}\right) \rightarrow 0$ the micro-motion operator defined in Eq. (5.54) is proportional to $\hat{n}_{\mathcal{R}}$ and does not influence the shape of the cyclotron orbits. In order to understand the corrections arising 

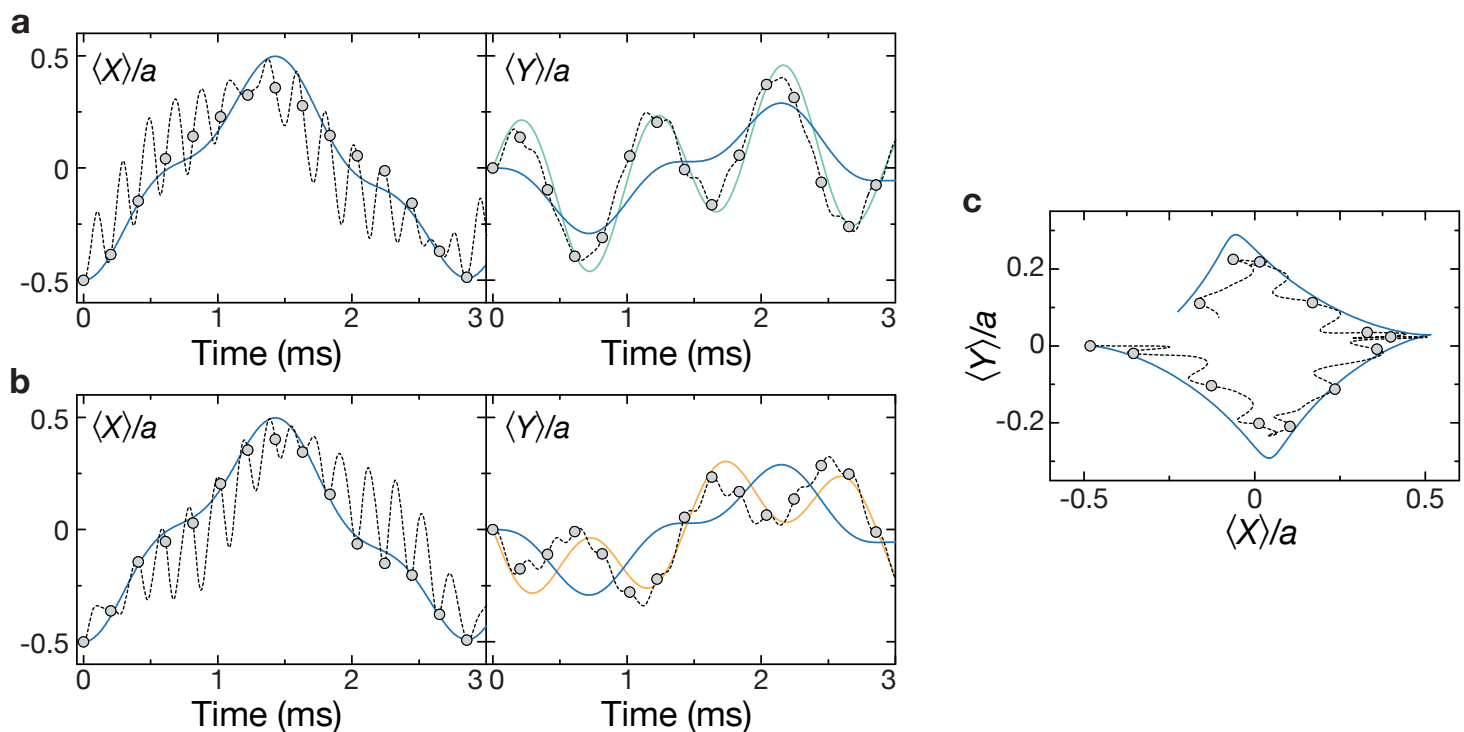

Figure 5.21: Comparison of the ideal cyclotron evolution $\langle X\rangle / a,\langle Y\rangle / a$ governed by the effective Floquet Hamiltonian with the exact full-time evolution and the impact of the initial kick of the modulation for $\Delta / J_{x}=3.56$. The exact time evolution (dashed line) was calculated using the time-dependent Hamiltonian (5.45), where the dots highlight the dynamics at stroboscopic times. The blue line depicts the ideal evolution governed by the effective Floquet Hamiltonian (5.42) using the initial state $\left|\psi_{1}\right\rangle$ and the green and orange lines illustrate the evolutions if the initial kick is taken into account as defined in Eqs. (5.55)-(5.56). The dynamics were calculated for the experimental parameters $V_{0} / \Delta=0.39, \mathrm{~K} / \mathrm{h}=0.28 \mathrm{kHz}, J / h=0.47 \mathrm{kHz}$ and $t \leq 3 \mathrm{~ms}$ used for the measurements shown in Fig. 5.17. The ratio between the bare coupling and the modulation frequency is $J_{x} /\left(\hbar \omega_{\text {res }}\right)=0.24$. a, b Calculated dynamics for the two kinds of plaquettes illustrated in Fig. 5.20c, which are realized simultaneously in the experiment. The initial phase was set to $\phi_{0}=-\pi / 4$. c Evolution averaged over the two realizations depicted in (a) and (b).

from a finite ratio $J_{x} / \Delta$ we consider again the evolution in the double-well potential along $x$. The first order corrections to the micro-motion operator $\hat{\mathcal{M}}_{P}(t)$ are of the form (Sect. 3.1.4)

$$
\mathcal{M}_{P}^{c}(t)=-\frac{J_{x}}{i \hbar \omega_{\text {res }}}\left(|1\rangle\left\langle 0\left|\mathrm{e}^{i \omega_{\text {res }} t}-\right| 0\right\rangle\langle 1| \mathrm{e}^{-i \omega_{\text {res }} t}\right) .
$$

This term couples the two sites or levels of the double-well $(|0\rangle$ and $|1\rangle)$ and gives rise to fast oscillations with period $T=2 \pi / \omega_{\text {res, }}$ whose amplitude scales with $J_{x} /\left(\hbar \omega_{\text {res }}\right)$.

In Figure 5.21 and 5.22 we show the full-time evolution of the cyclotron orbits according to Hamiltonian (5.45) for $\Delta / J_{x}=3.56$ and $\Delta / J_{x}=12$ respectively. To maintain the same effective coupling strengths $J$ and $K$ the modulation amplitude $V_{0}$ was increased accordingly for the calculations depicted in Fig. 5.22. The simulations reveal fast oscillations with frequency $\omega_{\text {res }}$, which are suppressed with increasing driving frequency. The ini- 

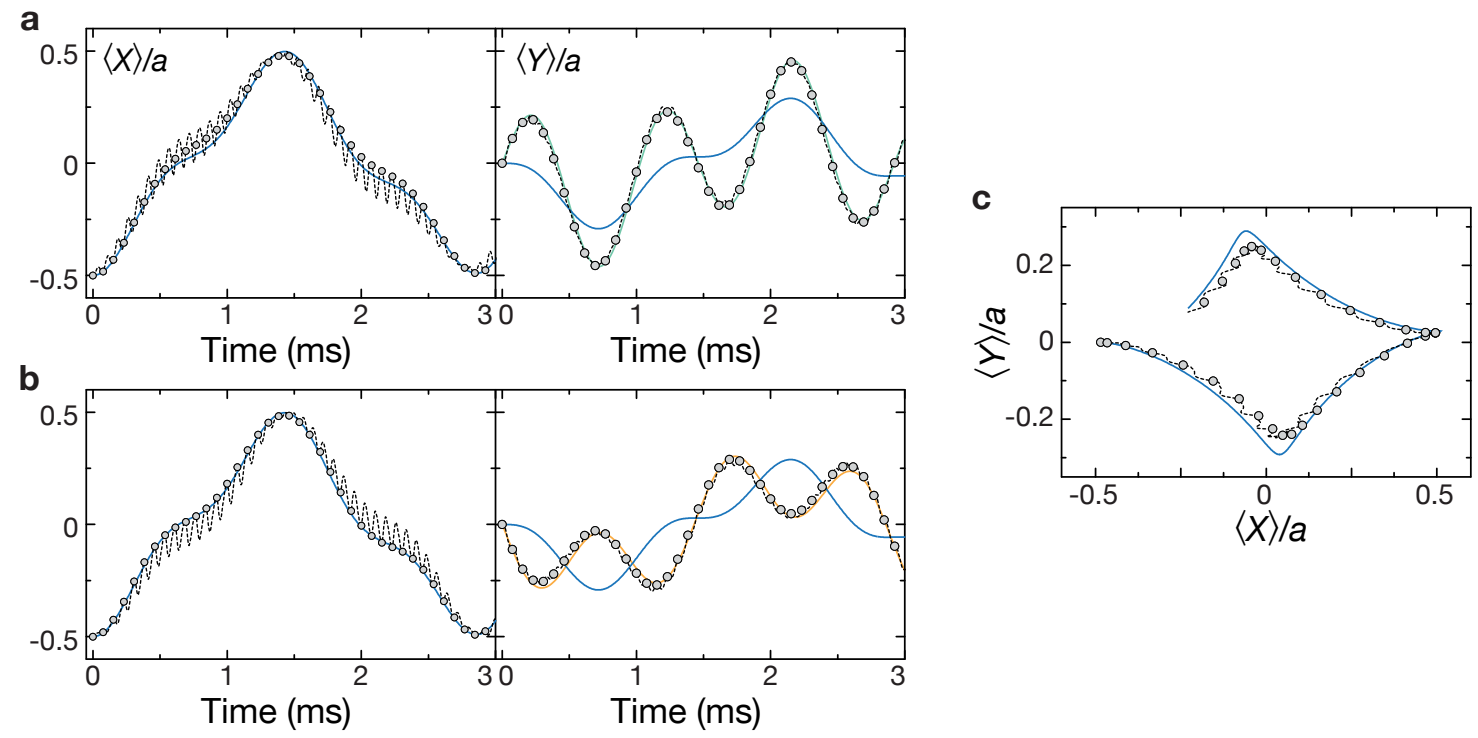

Figure 5.22: Comparison of the ideal cyclotron evolution $\langle X\rangle / a,\langle Y\rangle / a$ governed by the effective Floquet Hamiltonian with the exact full-time evolution and the impact of the initial kick for $\Delta / J_{x}=12$. The exact time evolution (dashed line) was calculated using Hamiltonian (5.45), where the dots highlight the dynamics at stroboscopic times. The blue line depicts the ideal evolution governed by the effective Floquet Hamiltonian (5.42) using the initial state $\left|\psi_{1}\right\rangle$ and the green and orange lines illustrate the corresponding evolutions taking into account the initial kick as defined in Eqs. (5.55)-(5.56). The parameters for the calculations were the same as in Fig. 5.21, only the ratio $\Delta / J_{x}=12$ was changed. The corresponding ratio between the bare coupling and the modulation frequency is $J_{x} /\left(\hbar \omega_{\text {res }}\right)=0.08$. a, b Calculated dynamics for the two kinds of plaquettes illustrated in Fig. 5.20c, which are realized simultaneously in the experiment. c Evolution averaged over the two realizations depicted in (a) and (b).

tial phase of the driving $\phi_{0}$ influences the evolution even in the infinite frequency limit because it scales with the ratio $V_{0} /\left(\hbar \omega_{\text {res }}\right)$, which was kept constant.

In the experiment we are able to observe the cyclotron orbits because we averaged over the two plaquette realizations illustrated in Fig. 5.20c. The simulations show, that the individual evolutions in the plaquettes are considerably different from the ideal evolution as depicted in Fig. 5.21a and b for $\phi_{0}=-\pi / 4$. Fortunately, the averaged evolutions (Fig. 5.21c and 5.22c) agree well with the effective Hamiltonian (5.42) even for the rather small ratio $\Delta / J_{x} \simeq 3.56$ as it was chosen for the measurements depicted in Fig. 5.17. 


\section{Chapter 6}

\section{Harper-Hofstadter model and spin Hall effect}

In the previous chapter it was shown both theoretically and experimentally that laserassisted tunneling based on a staggered superlattice potential allows for the realization of a staggered flux distribution [68]. Here we demonstrate a natural extension of the previously described method by replacing the staggered potential with a linear one; this gives rise to a uniform flux distribution $\Phi=\pi / 2$ [70], which is described by the famous Harper-Hofstadter Hamiltonian [74-76]. In general the strength of the flux $\Phi$ is fully tunable by changing the geometry of the laser-assisted tunneling scheme. Similar work was performed at MIT with a uniform flux $\Phi=\pi$ per plaquette [71]. By exploiting an additional pseudo-spin degree of freedom our setup further implements the timereversal symmetric Hamiltonian underlying the quantum spin Hall effect [15, 16]. The pseudo-spins are realized with two Zeeman states of opposite magnetic moment. Both spin states experience the same strength of the effective magnetic field but its direction is spin-dependent [70]. The derivation of the effective Hamiltonian for our experimental setup is demonstrated in Sect. 6.1. Using the previously developed technique of local cyclotron orbits [68] we were able to probe the spatial distribution of the artificial gauge field and to demonstrate the uniform nature of the flux (Sect. 6.3). The spin-dependent chirality of the observed cyclotron orbits verifies the spin-dependence of the artificial flux, which exhibits the same strength but points in opposite directions [77]. This further enabled us to observe the spin Hall effect in an optical lattice (Sect. 6.4). Recently, the free-space spin Hall effect was observed in quantum gases using Raman dressing [183]. 


\subsection{Experimental setup}

The laser-assisted tunneling setup used to generate a uniform flux distribution is very similar to the one described in Sect. 5.3. The only difference is, that tunneling along the $x$-direction is inhibited by a linear potential (Fig. 6.1a). This configuration will naturally lead to a uniform flux distribution if the phase of the on-site modulation increases linearly along the two axes of the 2D lattice potential. This is the case for the previous scheme, where two running-wave beams were applied as illustrated in Fig. 5.4. Each of the two beams was aligned along on of the principal axes of the $2 \mathrm{D}$ lattice thereby creating a running-wave interference term which exhibits phase fronts that are diagonal relative to the underlying lattice (Fig. 5.4c).

There are two possibilities for the implementation of a linear potential. The first one builds on optical dipole forces [158]: A Gaussian laser beam that is focused on the atom position such that the atoms are located at the maximum slope of the beam profile along $x$, while being centered along $y$, introduces an approximately linear potential along $x$ if the waist of the beam is large compared to the extent of the atomic cloud. The use of optical potentials has the advantage that they can be easily controlled experimentally and therefore offer a large experimental flexibility. The laser-assisted tunneling method, however, requires large energy offsets $\Delta$ between neighboring sites. Thus, large optical powers or near-resonant laser beams have to be employed. Both options increase heating in the system due to spontaneous emission of photons.

The second possibility is to use magnetic field gradients, which allow for a tuning of the energy offset $\Delta$ over a wide range without having to deal with increased heating due to spontaneous emission and maintaining the homogeneity of the linear potential across the atomic cloud. One possible disadvantage is the reduced experimental flexibility regarding the experimental controllability compared to optical fields. In the following discussion we are going to present an experimental setup based on a linear potential created using a magnetic field gradient, which offers an additional advantage, namely it naturally allows for the realization of a time-reversal symmetric Hamiltonian which underlies the quantum spin Hall effect $[15,16,77]$.

\subsubsection{Harper-Hofstadter model}

The experimental setup consists of a two-dimensional lattice created by two orthogonal standing waves with wavelength $\lambda_{s}=767 \mathrm{~nm}$ (Fig. 6.1). Along the $x$-direction a magnetic field gradient $B^{\prime}$ is applied to generate a linear potential $\Delta_{m}=\Delta m$, which inhibits tunneling along the corresponding direction for $\Delta \gg J_{x}$. Resonant tunneling is then restored with the setup discussed in detail in the previous chapter using two runningwave beams as defined in Eq. (5.18) and illustrated with the red arrows in Fig. 6.1a. The corresponding local optical potential can be written in the following form 


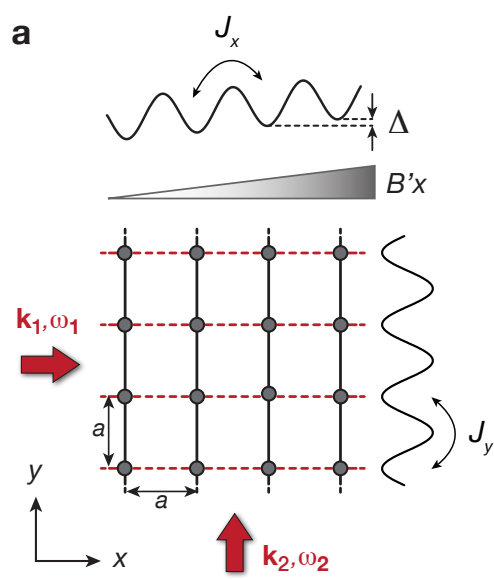

b

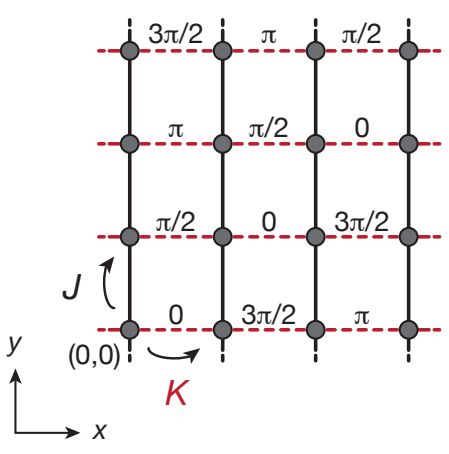

Figure 6.1: Experimental setup to generate a uniform flux distribution in a 2D square lattice and spatial distribution of the Peierls phase-factors. a The setup consists of a 2D lattice with constant $a=\lambda_{s} / 2$ and bare tunneling couplings $J_{x}$ and $J_{y}$. A magnetic field gradient $B^{\prime}$ along $x$ is used to introduce an energy offset between neighboring sites. Tunneling along $x$ is inhibited for $\Delta \gg J_{x}$. An additional pair of far-detuned running-wave beams (red arrows) with wave vectors $\left|\mathbf{k}_{1}\right| \simeq\left|\mathbf{k}_{2}\right| \simeq k_{l}=\pi /(2 a)$ restores resonant tunneling for $\omega=\omega_{1}-\omega_{2}=\Delta / \hbar$. $\mathbf{b}$ Phase distribution $\phi_{m, n}$ of the effective Hamiltonian (6.4). The induced coupling strengths are denoted as $K$ and $J$ respectively.

$$
V_{K}(\mathbf{r}, t)=V_{0} \cos \left(\delta \mathbf{k} \cdot \mathbf{r}+\omega t+\phi_{0}\right),
$$

with $\delta \mathbf{k}=\mathbf{k}_{1}-\mathbf{k}_{2}$ and $\omega=\omega_{1}-\omega_{2}$; the constant global energy shift was neglected. The site-dependent phase of the modulation is determined by

$$
\delta \mathbf{k} \cdot \mathbf{R}=\frac{\pi}{2}(m-n), \quad \mathbf{R}=m a \hat{\mathbf{e}}_{x}+n a \hat{\mathbf{e}}_{y} .
$$

and the time-dependent Hamiltonian of the system can be written as

$$
\begin{aligned}
\hat{H}(t) & =\sum_{m, n}\left(-J_{x} \hat{a}_{m+1, n}^{\dagger} \hat{a}_{m, n}-J_{y} \hat{a}_{m, n+1}^{\dagger} \hat{a}_{m, n}+\text { h.c. }\right) \\
& +\sum_{m, n}\left(V_{0} \sin \left(\omega t+\varphi_{m, n}\right)+\Delta m\right) \hat{n}_{m, n},
\end{aligned}
$$

with $\varphi_{m, n}=\frac{\pi}{2}(m-n+1)+\phi_{0}$. This Hamiltonian is time-periodic $\hat{H}(t+T)=\hat{H}(t)$, with $T=2 \pi / \omega$, such that in the high-frequency limit $\hbar \omega \gg J_{x}, J_{y}$ the formalism discussed in Sect. 3.4 can be directly applied. The corresponding effective time-independent Hamiltonian is written as 


$$
\begin{aligned}
& \hat{\mathcal{H}}_{F}=-K \sum_{m, n} \mathrm{e}^{i \phi_{m, n}} \hat{a}_{m+1, n}^{+} \hat{a}_{m, n}-J \sum_{m, n} \hat{a}_{m, n+1}^{+} \hat{a}_{m, n}+\text { h.c. }, \\
& \phi_{m, n}=\frac{\pi}{2}(n-m),
\end{aligned}
$$

with $K=J_{x} \mathcal{J}_{1}\left(\eta_{0}\right), J=J_{y} \mathcal{J}_{0}\left(\eta_{0}\right)$ and $\eta_{0}=\sqrt{2} V_{0} /(\hbar \omega)$. The spatial distribution of the Peierls phase-factors are illustrated in Fig. 6.1b. For the sake of simplicity we set $\phi_{0}=-3 \pi / 4$. This Hamiltonian is equivalent to the Hofstadter model for $\Phi=\pi / 2$. For the following discussion we are going to use the convention that the magnetic field is pointing in the $-\hat{\mathbf{e}}_{z}$ direction.

\subsubsection{Spin-dependent Harper-Hofstadter model}

The simplest setting where quantum spin Hall physics can be observed consists of two independent copies of a quantum Hall system, one for each spin state, $|\uparrow\rangle$ and $|\downarrow\rangle$, where the strength of the magnetic field is spin-independent but the direction of the field "is opposite" for the two states.

Such as system can be realized by introducing a pseudo-spin degree of freedom into the model described above. For the following experiments we use two different Zeeman states of ${ }^{87} \mathrm{Rb}$ with opposite magnetic moments to encode the pseudo-spin. The two hyperfine states are denoted as

$$
|\uparrow\rangle \equiv\left|F=1, m_{F}=-1\right\rangle \quad|\downarrow\rangle \equiv\left|F=2, m_{F}=-1\right\rangle .
$$

The linear potential $\Delta_{m}= \pm \Delta m$ implemented with a magnetic field gradient is now spin-dependent; the plus-sign corresponds to $|\uparrow\rangle$ particles (Fig. 6.2a) and the minus-sign to $|\downarrow\rangle$ particles (Fig. 6.2b). In Sect. 3.2 and 5.4 it was shown that the Peierls phase-factors $\pm \phi_{m, n}$ engineered with laser-assisted tunneling depend on the sign of the energy offset $\pm \Delta$ between neighboring sites. The total Hamiltonian for the two spin components can be written in the following form

$$
\hat{\mathcal{H}}_{\text {spin }}=\sum_{m, n}\left(-K \mathrm{e}^{i \hat{\phi}_{m, n}} \hat{a}_{m+1, n}^{\dagger} \hat{a}_{m, n}-J \hat{a}_{m, n+1}^{\dagger} \hat{a}_{m, n}+\text { h.c. }\right),
$$

where $\hat{\phi}_{m, n}=\phi_{m, n} \hat{\sigma}_{z}$ and $\hat{\sigma}_{z}$ is the $z$-component of the Pauli spin matrix $\vec{\sigma}$. Strictly speaking there is an additional phase shift of $\pi$ between the two spin-components (Sect. 3.2). However, since the two spin-states are not coupled it has no influence on the dynamics of the system and can be safely neglected. Hamiltonian (6.7) describes two independent copies of the Harper-Hofstadter model for $\Phi=\pi / 2$, where the two spin components experience a magnetic field of the same strength but opposite direction: $\Phi_{\uparrow}=\pi / 2$ for the $|\uparrow\rangle$ state and $\Phi_{\downarrow}=-\pi / 2$ for $|\downarrow\rangle$. In contrast to the Harper-Hofstadter Hamiltonian, 

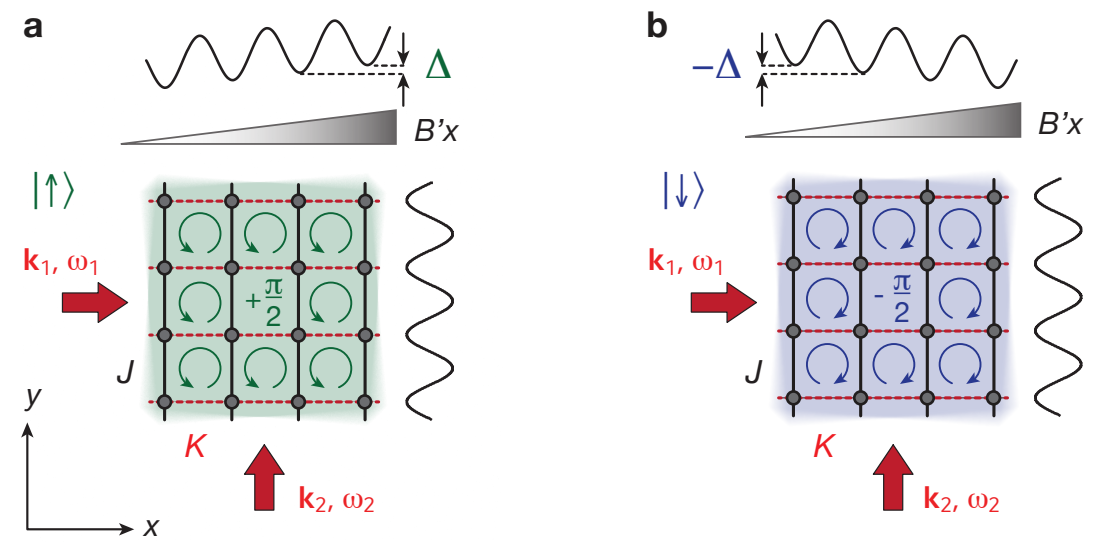

Figure 6.2: Implementation of the spin-dependent Harper-Hofstadter model using the setup illustrated in Fig. 6.1. Schematic drawing of the experimental setup for a $|\uparrow\rangle$ particles and b $|\downarrow\rangle$ particles. The two pseudo-spin components denote two different Zeeman states with opposite magnetic moment. Hence, the magnetic field gradient $B^{\prime}$ gives rise to a linear potential $\Delta_{m}= \pm \Delta m$, where the sign depends on the spin of the particle. As a result the direction of the effective magnetic field is spin-dependent: $\Phi_{\uparrow}=\pi / 2$ for $|\uparrow\rangle$ particles (a) and $\Phi_{\downarrow}=\pi / 2$ for $|\downarrow\rangle$ particles (b). (Figure adapted from [70])

which breaks time-reversal symmetry, this Hamiltonian is time-reversal symmetric. Including additional terms in the Hamiltonian which couple the two spin components would allow for the realization of non-Abelian gauge fields [184, 185].

\subsection{Laser-assisted tunneling in a tilted lattice potential}

In order to investigate the global properties of the system described above we studied laser-assisted tunneling in the presence of a magnetic field gradient $B^{\prime}$ in the 2D lattice by performing spectroscopy measurements (Sect. 4.3.2). The measured spectra are independent of the spin state because only the absolute value of the energy offset $|\Delta|$ determines if atoms can tunnel resonantly to the neighboring sites (inset in Fig. 6.3b). All measurements presented in the following section were performed with $|\uparrow\rangle$ particles. The experimental sequence started with an initial state where all atoms populated even sites along the $x$ direction of the lattice with at most one atom per lattice site and odd sites were left empty (Fig. 6.3a). The final lattice depths were $V_{x}=5.0(1) \mathrm{E}_{\mathrm{rs}}$ and $V_{y}=40(1) \mathrm{E}_{\mathrm{rs}}$. Due to the deep lattice along $y$, tunneling is inhibited along that direction on the timescales of the experiment, while bare tunneling along $x$ occurs with strength $J_{x} / h=0.26(1) \mathrm{kHz}$. Additionally a magnetic field gradient was applied, which inhibited tunneling along $x$, such that all dynamics was frozen and atoms stayed in even lattice sites. Perpendicular to the 2D plane an additional lattice potential was used with wavelength $\lambda_{z}=844 \mathrm{~nm}$ in order to isolate individual planes from each other. The final 

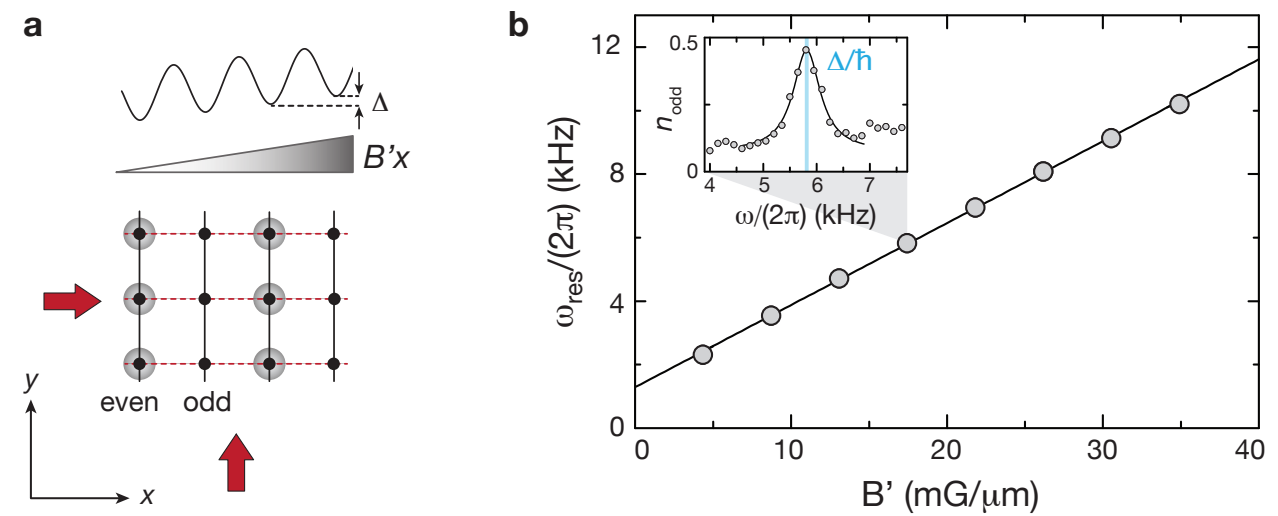

Figure 6.3: Laser-assisted tunneling in a tilted optical lattice. a Illustration of the initial state, where all atoms (gray spheres) populate even sites with at most one atom per lattice site and odd sites are left empty. $\mathbf{b}$ Measured resonance frequency $\omega=\omega_{1}-\omega_{2}$ obtained from spectroscopy measurements (blue vertical line in the inset) as a function of the magnetic field gradient $B^{\prime}$. The offset at $B^{\prime}=0$ is due to an additional magnetic field gradient present in the experiment (see main text). The solid line is a linear fit to the data. The inset shows a typical spectroscopy measurement for $B^{\prime}=17.5 \mathrm{mG} / \mu \mathrm{m}$. For the modulation frequency $\omega=\Delta / \hbar$ atoms can tunnel resonantly to odd sites and the transferred fraction of atoms $n_{\text {odd }}$ exhibits a maximum. The solid line depicts the fit of a Lorentzian to the data used to extract the resonance frequency. (Data taken from Ref. [70])

lattice depth was $V_{z}=30(1) \mathrm{E}_{\mathrm{rz}}$. After switching on the modulation abruptly for a duration of $t=4 \mathrm{~ms}$ with strength $V_{0}=2.48(5) \mathrm{E}_{\mathrm{rl}}$ the fraction of atoms transferred to odd sites $n_{\text {odd }}=N_{\text {odd }} / N$ was measured as a function of the modulation frequency $\omega$; here $N$ denotes the total atom number. The even-odd resolved detection was achieved by applying the site-resolved detection technique along $x$ as discussed in Sect. 4.6 ( $n_{\text {odd }} \widehat{=} n_{\text {right }}$ ). If the frequency of the modulation $\omega$ is resonant with the energy offset between neighboring sites $\omega=\Delta / \hbar$ the transferred fraction $n_{\text {odd }}$ exhibits a maximum (inset Fig. 6.3b), which allows us to determine the energy offset $\Delta$ between neighboring sites produced by the magnetic field gradient. Figure $6.3 \mathrm{~b}$ displays the measured resonance frequency $\omega$ as a function of the magnetic field gradient $B^{\prime}$. It shows that the energy offset $\Delta$ can be adjusted over a large range of values up to $\Delta / h \sim 10 \mathrm{kHz}$.

\section{Initial state preparation: Patterned loading}

The initial state illustrated in Fig. 6.3a was prepared with the help of an additional superlattice potential along $x$. The sequence started by loading a BEC into a 3D lattice in the MI regime with at most two atoms per lattice site [44]. The potential was created by the long lattice along $x, V_{x l}=30(1) \mathrm{E}_{\mathrm{rl}}$, the short lattice along $y, V_{y}=20.0(6) \mathrm{E}_{\mathrm{rs}}$, and the vertical lattice with depth $V_{z}=20.0(6) \mathrm{E}_{\mathrm{rz}}$. In order to empty all doubly occupied sites a filtering sequence was applied (Sect. 4.5), for which the lattice depths 
were increased to $V_{x l}=104(3) \mathrm{E}_{\mathrm{rl}}, V_{y}=100(3) \mathrm{E}_{\mathrm{rs}}$ and $V_{z}=120(3) \mathrm{E}_{\mathrm{rz}}$. After all doubly occupied sites were removed from the trap the lattices were ramped down again to $V_{x l}=52(2) \mathrm{E}_{\mathrm{rl}}, V_{y}=40(1) \mathrm{E}_{\mathrm{rs}}$ and $V_{z}=30(1) \mathrm{E}_{\mathrm{rz}}$. Subsequently, the magnetic field gradient was switched on within $200 \mathrm{~ms}$. The ramping time was chosen large enough to assure that the gradient has reached its final value. As a last step the short lattice along $x$ was ramped up within $20 \mathrm{~ms}$ to $V_{x}=40(1) \mathrm{E}_{\mathrm{rs}}$ with $\varphi_{S L}^{x}=0$ (Sect. 4.3) in the symmetric double-well configuration in order to load all atoms into the lower energy sites of the tilted double-well potential; the energy offset between neighboring sites in the double-well is introduced by the magnetic field gradient. After switching off the long lattice within $2 \mathrm{~ms}$ and lowering the short lattice to its final value $V_{x}=5.0(1) \mathrm{E}_{\mathrm{rs}}$ the initial state for the measurements depicted in Fig. 6.3b was obtained.

\section{Realization of the magnetic field gradient}

The magnetic field gradient $B^{\prime}$ was created using a quadrupole magnetic field in combination with an additional homogeneous offset field. In our experimental setup the quadrupole field is typically used to compensate gravity along the z-direction. In the horizontal plane the quadrupole field is aligned on the atom position in order to avoid possible magnetic field gradients along the $x$ and $y$ directions. To displace the minimum of the quadrupole magnetic field an additional offset field $B_{0}$ was applied. This induced a magnetic field gradient $B_{\text {quad }}^{\prime}(I)$ whose strength could be controlled with the current $I$ in the quadrupole coils. The offset field along $x$, however, is not entirely homogeneous but further involves a small magnetic field gradient $B_{0}^{\prime}$. Thus, the total gradient is given by the sum of the two: $B^{\prime}=B_{\text {quad }}^{\prime}(I)+B_{0}^{\prime}$. From the data shown in Fig. $6.3 \mathrm{~b}$ the offset gradient $B_{0}^{\prime}$ could be evaluated, which corresponds to $\Delta / h=1.30(4) \mathrm{kHz}$.

\subsection{Probing the magnetic flux distribution}

The local structure of the artificial magnetic field can be examined on the level of isolated four-site square plaquettes by tracking the evolution of single atoms inside these plaquettes. This technique has been developed previously to probe the strength of the magnetic field for the staggered flux distribution studied in Sect. 5.6 and Ref. [68, 69]. The evolution after quenching the system from a plaquette with zero flux to $\Phi=\pi / 2$ is reminiscent of a cyclotron orbit a charged particle would follow in the presence of a magnetic field. The full-time dynamics of the real-space dynamics was discussed in detail in Sect. 5.7. In conclusion it was found that the cyclotron dynamics can be described with the effective time-independent Hamiltonian in the rotating frame and the micro-motion can be safely neglected for our parameter regime. In the following measurements the same method will be applied to explore the spatial distribution of the artificial magnetic field in the 2D lattice.

The experiments were performed in a 3D optical lattice. In the horizontal plane two su- 
perlattice potentials were employed in the symmetric double-well configuration, $\varphi_{S L}^{x}=$ $\varphi_{S L}^{y}=0$ (Sect. 4.3), in order to inhibit tunneling on every other bond along both directions such that all dynamics is restricted to the four sites of a plaquette potential. The final lattice depths were $V_{x}=7.0(2) \mathrm{E}_{\mathrm{rs}}, V_{y}=10.0(3) \mathrm{E}_{\mathrm{rs}}$ and $V_{x l}=V_{y l}=35(1) \mathrm{E}_{\mathrm{rl}}$. Along the vertical axis an additional lattice was used to isolate individual planes; the corresponding lattice depth was $V_{z}=30(1) \mathrm{E}_{\mathrm{rz}}$. Furthermore tunneling along $x$ in the plaquette was inhibited by the magnetic field gradient $\Delta \gg J_{x}$, where $J_{x}$ denotes the bare inner-well coupling of the double-well potential along $x$.

As an initial state we prepared single atoms in the ground state of the tilted plaquettes as illustrated by the gray shaded area in Fig. 6.4i. Due to the spin-dependency of the energy offset $\pm \Delta$ between neighboring sites along $x$, the ground states are spin-dependent as well: $\left|\Psi_{\uparrow}\right\rangle=(|A\rangle+|D\rangle) / \sqrt{2}$ for $|\uparrow\rangle$ particles (Fig. 6.4a) and $\left|\Psi_{\downarrow}\right\rangle=(|B\rangle+|C\rangle) / \sqrt{2}$ for $|\downarrow\rangle$ particles (Fig. 6.4b). The evolution was then triggered by switching on the modulation instantaneously with $\omega=\Delta / \hbar$ and thereby quenching the system from tilted plaquette potentials with $\Phi=0$ to symmetric ones with $\Phi= \pm \pi / 2$. The energy offset $\Delta$ was calibrated independently through spectroscopy measurements (Sect. 4.3.2). The dynamics of a single atom in the plaquette is governed by the following effective $4 \times 4$ Hamiltonian written in the basis $\{|A\rangle,|B\rangle,|C\rangle,|D\rangle\}$

$$
\hat{H}_{\uparrow, \downarrow}^{P}=-\left(\begin{array}{cccc}
0 & K & 0 & J \\
K & 0 & J & 0 \\
0 & J & 0 & \operatorname{Kexp}\left[-i \Phi_{\uparrow, \downarrow}\right] \\
J & 0 & K \exp \left[i \Phi_{\uparrow, \downarrow}\right] & 0
\end{array}\right) .
$$

Using the site-resolved detection techniques introduced in Sect. 4.6 we evaluated the mean atom positions $\langle X\rangle,\langle Y\rangle$ within the plaquettes,

$$
\langle X\rangle=\frac{\left(N_{\text {right }}-N_{\text {left }}\right) a}{2 N}, \quad\langle Y\rangle=\frac{\left(N_{\text {up }}-N_{\text {down }}\right) a}{2 N},
$$

where $N_{\text {left }}=N_{A}+N_{D}, N_{\text {right }}=N_{B}+N_{C}, N_{\text {up }}=N_{C}+N_{D}, N_{\text {down }}=N_{A}+N_{B}$ and $N_{\mathcal{R}}$ is the atom number on each site $\mathcal{R}$ of the plaquette.

Without the magnetic field the atoms would start to undergo Rabi oscillations between left $(A$ and $D)$ and right $(B$ and $C$ ) sites, however, due the the artificial gauge field the atoms get deflected perpendicular to their direction of motion, which is reminiscent of the Lorentz force acting on a charged particle in a magnetic field (Fig. 6.4). We observe that $|\uparrow\rangle$ particles start to to tunnel to the right and get deflected to the lower bond while $|\downarrow\rangle$ particles first tunnel to the left and get deflected to the same bond as $|\uparrow\rangle$ particles. Hence, the two orbits exhibit opposite chirality, which is a manifestation of the spindependent direction of the flux $\Phi= \pm \pi / 2$.

The observation of local cyclotron orbits is equivalent to the results obtained in a lattice with staggered flux $\Phi=(-1)^{m} \pi / 2$ because in these measurements only plaquettes with equal sign of the flux contributed (Sect. 5.6). In order to probe the uniform nature 
a i

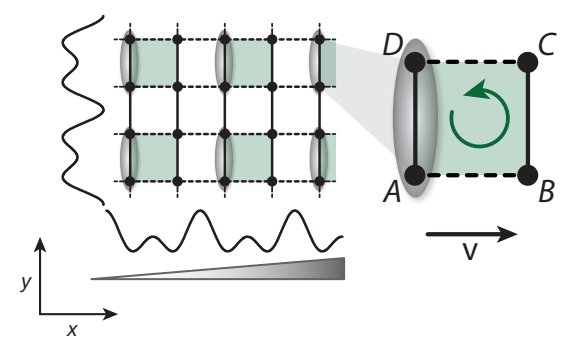

b i

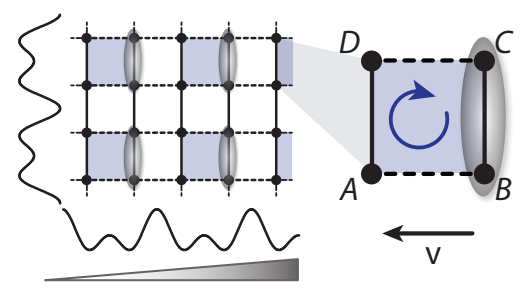

ii

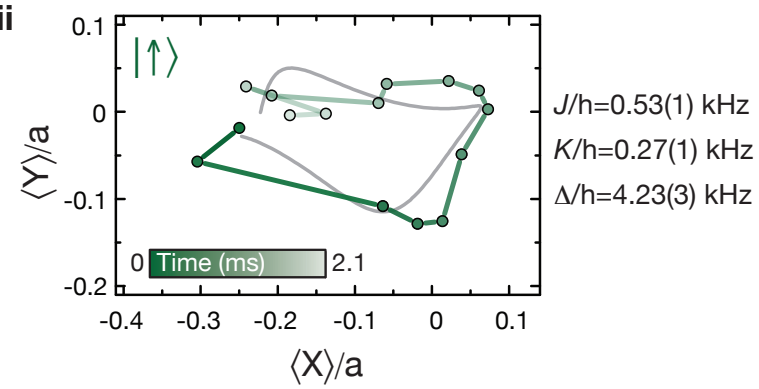

ii

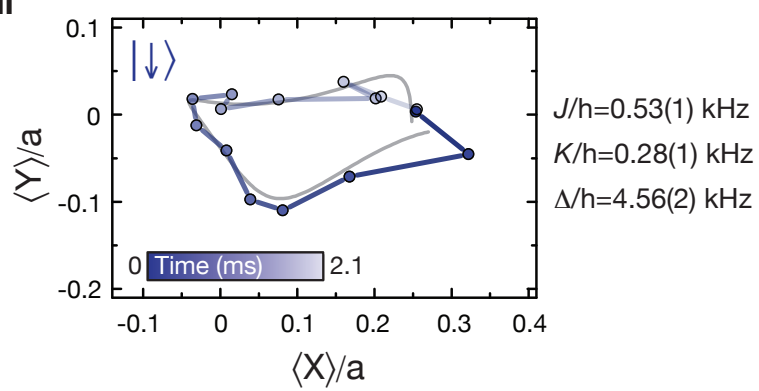

Figure 6.4: Probing the spin-dependence of the artificial gauge field with local cyclotron orbits for $J / K \approx 2$. $\mathbf{i}$ The setup consists of a $2 \mathrm{D}$ superlattice that consists of symmetric double-well potentials along $x$ and $y$. The high potential barrier on every other bond suppresses interdouble-well tunneling so that all dynamics is restricted to the four sites of each plaquette. The corresponding lattice sites are denoted as $\mathcal{R}=\{A, B, C, D\}$. The initial state is a single atom in the ground state of the tilted plaquette (gray shaded area): $\left|\Psi_{\uparrow}\right\rangle=(|A\rangle+|D\rangle) / \sqrt{2}$ for $|\uparrow\rangle$ (a) and $\left|\Psi_{\downarrow}\right\rangle=(|B\rangle+|C\rangle) / \sqrt{2}$ for $|\downarrow\rangle$ particles (b). ii Evolution of the mean atom position in the plaquette for $|\uparrow\rangle$ (a) and $|\downarrow\rangle$ particles (b). The solid gray line is a fit to our data of the numerically calculated dynamics $f_{X}(t)$ and $f_{Y}(t)$ defined in Eq. (6.10), which were obtained with Hamiltonian (6.8). The time offset and the flux were set to $\tau=0.12(5) \mathrm{ms}$ and $\Phi=0.73(5) \times \pi / 2$ respectively; $X_{0}, Y_{0}, A_{\langle X\rangle}$ and $A_{\langle Y\rangle}$ were free fit parameters. Each data point is an average over three individual measurements. The opposite chirality of the orbits is a manifestation of the spin-dependent direction of the flux $\Phi= \pm \pi / 2$. (Data taken from Ref. [70])

of the artificial gauge field the superlattice potential along $x$ was shifted by one lattice constant (Fig. 6.5i). This was achieved by changing the relative phase between the short and the long lattice from $\varphi_{S L}^{x}=0$ to $\varphi_{S L}^{x}=\pi$ (Sect. 4.3). The measurements were performed in exactly the same way as described above but this time the neighboring plaquettes along $x$ participated in the measurements; these are the plaquettes which did not contribute to the experimental results shown in Fig. 6.4. Again we observe orbits with opposite chirality for the two spin states (Fig. 6.5) and moreover the chirality is the same as in the measurements depicted in Fig. 6.4. This shows that the direction of the flux is unchanged between adjacent plaquettes, which is consistent with a uniform flux distribution.

The experimental data was fitted with the theoretical evolution discussed below, which 
a $\mathbf{i}$

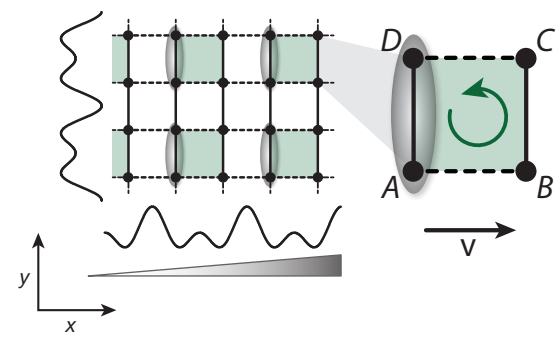

b i

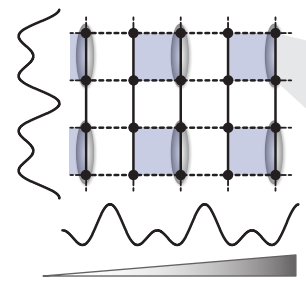

ii

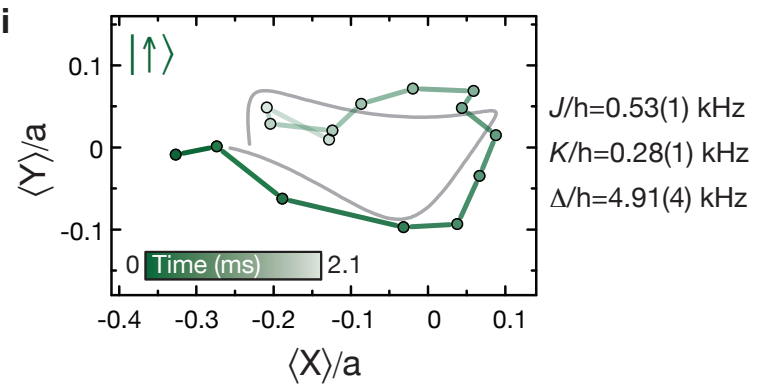

ii

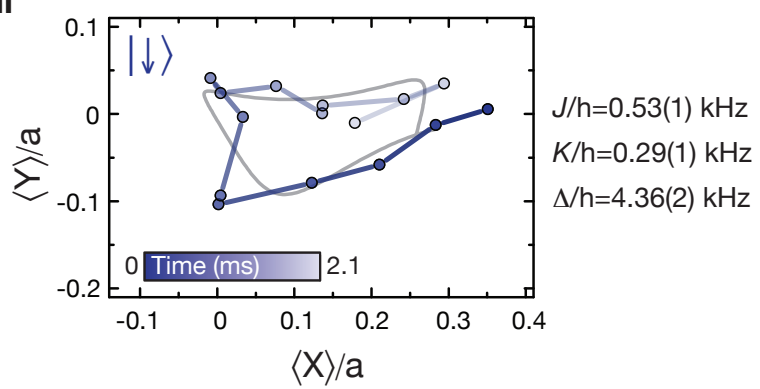

Figure 6.5: Flux distribution obtained from local cyclotron orbits in adjacent plaquettes for $J / K \approx 2$. i Schematic drawing of the setup for $|\uparrow\rangle$ particles (a) an $|\downarrow\rangle$ particles (b) as in Fig. 6.4. The superlattice potential along $x$ is shifted by one lattice constant such that all dynamics is now restricted to the neighboring plaquettes which did not participate in the measurements presented in Fig. 6.4. ii Measured mean atom positions $\langle X\rangle$ and $\langle Y\rangle$ versus modulation time. The solid gray line is a fit to our data of the numerical evolutions $f_{X}(t)$ and $f_{Y}(t)$ defined in Eq. (6.10), which were obtained with Hamiltonian (6.8). The time offset was $\tau=0.12(5) \mathrm{ms}$, the flux $\Phi=0.73(5) \pi / 2$ and $X_{0}, Y_{0}, A_{\langle X\rangle}$ and $A_{\langle Y\rangle}$ were free fit parameters. Every data point is an average over three individual measurements. The orbits exhibit opposite chirality depending on the spin but they are the same compared to the ones displayed in Fig. 6.4. (Data taken from Ref. [70])

was obtained by solving the Schrödinger equation associated with Hamiltonian (6.8) numerically using the previously determined value of the magnetic flux per plaquette $\Phi=0.73(5) \times \pi / 2$ (Sect. 5.6 and $[68,69]$ ). The smaller amplitude of the cyclotron orbits compared to the theoretical prediction is most likely due to inhomogeneities in the system caused by the external harmonic confinement. The reduced value of the flux $\Phi=0.73(5) \times \pi / 2$ stems from the smaller distance between lattice sites along $y$ when the lattice is partitioned into an array of isolated plaquette potentials (Fig. 5.18).

\subsubsection{Experimental sequence}

The experimental sequence was similar to the one illustrated in Fig. 5.15. It started by loading a BEC into a 3D lattice potential with depths $V_{x l}=V_{y l}=20.0(6) \mathrm{E}_{\mathrm{rl}}$ and $V_{z}=$ 20.0(6) $\mathrm{E}_{\mathrm{z}}$ in the Mott-insulating regime with at most two atoms per lattice site. Then, a 
filtering sequence (Sect. 4.5) was applied to remove all atoms on doubly-occupied sites from the trap. At this point of the sequence all atoms were in the $|\uparrow\rangle=\left|F=1, m_{F}=-1\right\rangle$ state and all lattice sites were populated with at most one atom. By removing the last Landau-Zener sweep from the filtering sequence, after all double occupancies were removed, all atoms remain in the $|\downarrow\rangle=\left|F=2, m_{F}=-1\right\rangle$ state with at most one atom per lattice site. During the filtering sequence the lattices were increased to much larger values typically around $V=100 \mathrm{E}_{\mathrm{r}}$ in order to enhance the scattering rate. After that we lowered them again to $V_{x l}=V_{y l}=35(1) \mathrm{E}_{\mathrm{rl}}$ and $V_{z}=30(1) \mathrm{E}_{\mathrm{rz}}$ within $1 \mathrm{~ms}$. In previous experiments the tilt was introduced by the superlattice phase $\varphi_{S L}^{x}$ (Sect. 5.6). For the measurement presented in this chapter a magnetic field gradient was applied along $x$. It was switched on in $250 \mathrm{~ms}$ together with the offset field $B_{0}$, which shifts the position of the quadrupole trap relative to the atom position (see discussion above). By ramping up the short lattice along $x$ within $15 \mathrm{~ms}$ the long-lattice sites were split into tilted double-well potentials with $V_{x}=40(1) \mathrm{E}_{\mathrm{rs}}$ and all particles occupied the sites with lower energy. Subsequently, the long lattice along $y$ was split within $0.1 \mathrm{~ms}$ by ramping up the short lattice to $V_{y}=10.0(3) \mathrm{E}_{\mathrm{rs}}$. Simultaneously the barrier of the double-well potential along $x$ was lowered to $V_{x}=7.0(2) \mathrm{E}_{\mathrm{rs}}$. The evolution started by switching on the modulation instantaneously on resonance. The final trap parameters $J, K$ and $\Delta$ were calibrated independently for each measurement (Sect. 4.3.2 and 4.4). The corresponding values are displayed in Fig. 6.4 and 6.5 respectively. For the calibration of the renormalized coupling $J=J_{x} \mathcal{J}_{0}\left(\sqrt{2} V_{0} / \Delta\right)$ a similar method was used as for the calibration of the induced tunneling coupling $K$. We measured Rabi oscillations between the left and right side of a symmetric isolated double-well potential in the presence of the modulation with driving frequency $\omega=\Delta / \hbar$.

\subsubsection{Numerical simulations}

We simulated the dynamics of the mean atom positions $\langle X\rangle$ and $\langle Y\rangle$ within the plaquettes by solving the time-dependent Schrödinger equation associated with Hamiltonian (6.8) numerically with $\Phi=0.73(5) \times \pi / 2$ (Sect. 5.6 and $[68,69]$ ). The numerical evolutions were fitted to our data using the following functions

$$
f_{X}(t)=X_{0}+A_{\langle X\rangle} \cdot X(t+\tau), \quad f_{Y}(t)=Y_{0}+A_{\langle Y\rangle} \cdot Y(t+\tau),
$$

where the offsets $X_{0} / Y_{0}$ and oscillations amplitudes $A_{\langle X\rangle} / A_{\langle Y\rangle}$ were free fit variables. The time offset $\tau$ was introduced to account for the finite ramping times that occur during the last ramps before the detection where we increased all lattice depths in order to freeze the dynamics. The ramping times are typically around $0.1-0.2 \mathrm{~ms}$, which prevents us from an exact determination of the $t=0$ point a priori. The offset $\tau$ was determined by fitting the numerical evolution for each data set individually and including $\tau$ as a fitting variable. We obtained an average value $\tau=0.12(5) \mathrm{ms}$ from the evolutions depicted in Fig. 6.4 and 6.5 . 

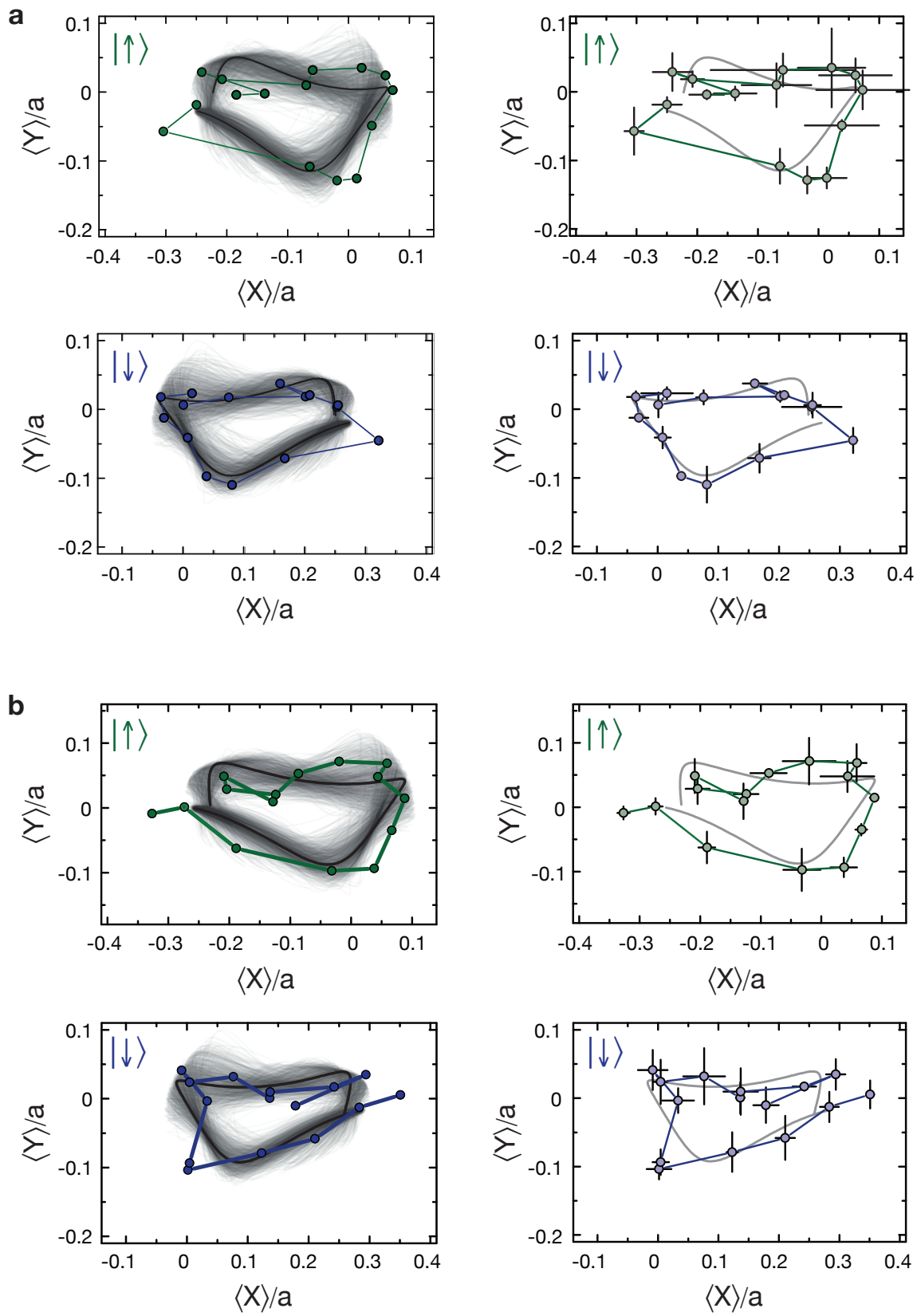

Figure 6.6: Numerical simulations based on statistical sampling of the parameter space. Comparison between the 1000 trajectories obtained from the Monte-Carlo samples described in the text and the measured cyclotron orbits (blue and green) displayed in Fig. 6.4 (a) and 6.5 (b). The black (left panels) and gray (right panels) solid lines show the fit to our experimental data using Eq. (6.10). The panels on the right depict the same data together with the error bars, which illustrate the standard deviation obtained from the individual measurements. (Data taken from Ref. [70]) 
a

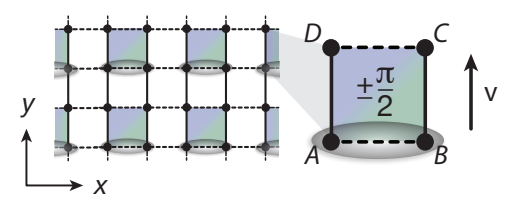

b

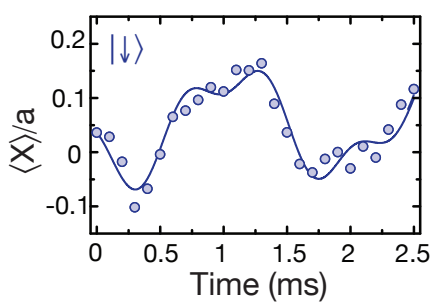

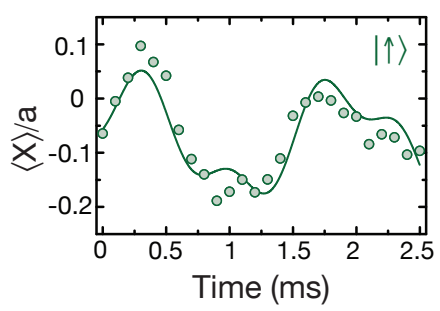

Figure 6.7: Initial state and spin-dependent oscillations transverse to the initial motion of the atoms. a Schematic drawing of the setup, which is an array of isolated plaquette potentials. The atoms were prepared in a spin-superposition state $|\psi\rangle_{\text {spin }}=\alpha|\uparrow\rangle+\beta|\downarrow\rangle$ before they were loaded into the ground state of the lower bond in the plaquette. $\mathbf{b}$ Mean atom position $\langle X\rangle$ for a spin-polarized sample with $\beta=1$ (left panel) and $\alpha=1$ (right panel) respectively. The experimental parameters were $J / h=0.69(1) \mathrm{kHz}, K / h=0.38(1) \mathrm{kHz}$ and $\Delta / h=5.31(5) \mathrm{kHz}$. The blue and green solid lines are a fit of the numerically determined evolution $f_{X}(t)$ defined in Eq. (6.10) to our data, the time offset was $\tau=0.18$ (3) ms. (Data taken from Ref. [70])

To gain better insight into the agreement between our experimental data and the numerical predictions we performed additional simulations taking into account the experimental uncertainties in the independently calibrated values $J, K$ and $\Phi$. We also considered possible detunings $\delta_{x}=\delta_{y}=0(30) \mathrm{Hz}$ along the $x$ and $y$ directions, which might be caused by an imperfect experimental control over the relative phase between the short and the long lattices $\varphi_{S L}^{x}$ and $\varphi_{S L}^{y}$. We assumed a normal distribution for each parameter $J, K, \Phi$ and $\delta_{x} / \delta_{y}$ with the mean and standard deviation values stated above. From these distributions a sample of random parameters is generated, which is then used to model the dynamics according to the following Hamiltonian

$$
\hat{H}_{\uparrow, \downarrow}^{P}=-\left(\begin{array}{cccc}
0 & K & 0 & J \\
K & -\delta_{x} & J & 0 \\
0 & J & -\left(\delta_{x}+\delta_{y}\right) & K \exp \left[-i \Phi_{\uparrow, \downarrow}\right] \\
J & 0 & K \exp \left[i \Phi_{\uparrow, \downarrow}\right] & -\delta_{y}
\end{array}\right)
$$

written in the basis $\{|A\rangle,|B\rangle,|C\rangle,|D\rangle\}$, where the sites of the plaquette are labeled as illustrated in Fig. 6.4 and 6.5. Such a sampling procedure is sometimes referred to as Monte-Carlo sampling. This procedure was repeated for 1000 samples and plotted together with our experimental data (left panels in Fig. 6.6). The amplitudes $A_{\langle X\rangle} / A_{\langle Y\rangle}$, offsets $X_{0} / Y_{0}$ and time delay $\tau$ were set to the values obtained from the fitting functions given above. For a better comparison with the data we further show the statistical uncertainties obtained from the individual measurements (right panels in Fig. 6.6). 


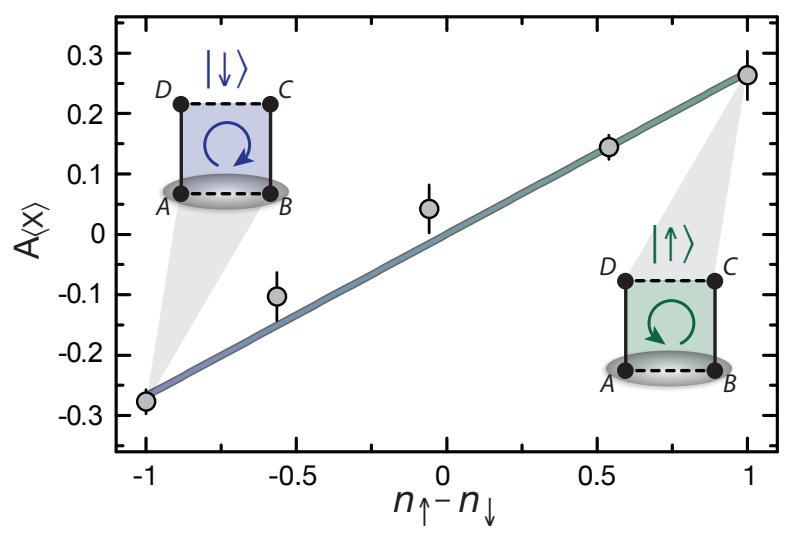

Figure 6.8: Oscillation amplitude $A_{\langle X\rangle}$ of the mean atom position $\langle X\rangle / a$ as a function of the spin-imbalance $n_{\uparrow}-n_{\downarrow}$. The total oscillation amplitude $A_{\langle X\rangle}=A_{\langle X\rangle}^{\downarrow}+A_{\langle X\rangle}^{\uparrow}$ was fitted according to the numerical evolution $f_{X}(t)$ defined in Eq. (6.10) with $\tau=0.18(3)$ ms. Every data point is an average over two individual measurements. The schematic drawings illustrate the initial state and the direction of the flux for fully polarized samples, $|\psi\rangle_{\text {spin }}=|\downarrow\rangle$ (blue) and $|\psi\rangle_{\text {spin }}=|\uparrow\rangle$ (green). The solid line is a linear fit to the data, where the offset was set to zero. The error bars depict the standard deviation obtained from the individual measurements. (Data taken from Ref. [70])

\subsection{Spin Hall effect in an optical lattice}

The spin Hall effect refers to a situation where a transverse spin current develops as a response to an electric field. It can be thought of as spin-up electrons moving transverse to the electric field in one direction and spin-down electrons moving in the opposite direction. In this case no net charge current flows but a spin imbalance builds up at the edges of the sample similar to the Hall voltage in the conventional Hall effect. This effect has been observed in thin-film semiconductor devices [186, 187] and with ultracold atoms in a harmonic trap using Raman dressing [183].

In this section related experimental results are presented which were obtained for the first time with ultracold bosonic atoms in optical lattices [70]. We determine the oscillation of single atoms in isolated plaquettes transverse to their initial motion as a function of the spin imbalance $n_{\uparrow}-n_{\downarrow}$, where $n_{\uparrow}=N_{\uparrow} / N$ is the fraction of atoms with spin-up and $n_{\downarrow}=N_{\downarrow} / N$ the one with spin-down.

The experimental setup was similar to the one described above, which consisted of a 3D optical lattice that was partitioned into isolated plaquettes in the horizontal plane using superlattice potentials (Fig. 6.7a). Contrary to the previous sequence we prepared the atoms in a spin-superposition state $|\psi\rangle_{\text {spin }}=\alpha|\uparrow\rangle+\beta|\downarrow\rangle$ before we loaded them into the ground state of the lower bond in the plaquette potential. This state has equal weight on $A$ and $B$ sites independent of the spin state $|\psi\rangle_{\text {spin }}$. We measured the mean atom position in the non-trivial direction $\langle X\rangle / a$ and observed almost perfectly mirrored oscillations 
for the two spin states (Fig. 6.7b). We obtained $A_{\langle X\rangle}=-0.28(2)$ for $|\psi\rangle_{\text {spin }}=|\downarrow\rangle$ (blue) and $A_{\langle X\rangle}=0.26(4)$ for $|\psi\rangle_{\text {spin }}=|\uparrow\rangle$ (green). The measured oscillation amplitude $A_{\langle X\rangle}=A_{\langle X\rangle}^{\downarrow}+A_{\langle X\rangle}^{\uparrow}$ was found to depend linearly on the spin imbalance $n_{\uparrow}-n_{\downarrow}$ and it reversed the sign when the spin was flipped (Fig. 6.8).

\section{Experimental sequence}

The sequence started by loading a BEC into a deep 3D optical lattice in the MI-regime created by the two long lattices and the vertical one. The final depths were $V_{x l}=$ $30(1) \mathrm{E}_{\mathrm{rl}}, V_{y l}=20.0(6) \mathrm{E}_{\mathrm{rl}}$ and $V_{z}=20.0(6) \mathrm{E}_{\mathrm{z}}$. For the filtering sequence (Sect. 4.5) all lattices were ramped up to $V_{x l}=104(3) \mathrm{E}_{\mathrm{rl}}, V_{y l}=70.0(2) \mathrm{E}_{\mathrm{rl}}$ and $V_{z}=120.0(4) \mathrm{E}_{\mathrm{z}}$ in order to increase the scattering rate. At the end of the filtering sequence the atoms are prepared in a spin-superposition state $|\psi\rangle_{\text {spin }}$ as described below. Subsequently the lattices were decreased to $V_{x l}=52(2) \mathrm{E}_{\mathrm{rl}}, V_{y l}=35(2) \mathrm{E}_{\mathrm{rl}}$ and $V_{z}=30(1) \mathrm{E}_{\mathrm{z}}$. In order to load the atoms into the lower bond of the plaquette the short lattice along $y$ was switched on within $20 \mathrm{~ms}$ to $V_{y}=40(2) \mathrm{E}_{\mathrm{rs}}$ with $\varphi_{S L}^{y} \neq 0$ (Sect. 4.3), which corresponds to a tilted double-well potential along $y$. Due to the tilt all atoms occupied the lower energy sites of the double-well (lower bond). In addition, the high potential barrier inhibited tunneling such that the relative phase could be changed adiabatically to $\varphi_{S L}^{y}=0$ within $40 \mathrm{~ms}$ resulting in a symmetric double-well configuration. Subsequently the magnetic field gradient was ramped up in $250 \mathrm{~ms}$ and after that the short lattice along $x$ was ramped up in $2 \mathrm{~ms}$ to its final value $V_{x}=6.0(2) \mathrm{E}_{\mathrm{rs}}$. In the presence of the magnetic field gradient $|\uparrow\rangle$ atoms are preferentially located on $A$ sites and $|\downarrow\rangle$ atoms on $B$ sites. Tunneling was inhibited along both directions due to the magnetic field gradient along $x$ and the high potential barrier along $y$. At this point the running-wave beams were switched on adiabatically within $7 \mathrm{~ms}$ to couple $A$ and $B$ sites. The potential barrier along $y$ remained high and the atoms where loaded into the ground state of the lower bond in the plaquette with equal weight on $A$ and $B$ sites. The cyclotron motion was then initiated by decreasing the short lattice along $y$ within $100 \mu$ s to $V_{y}=9.0(3) \mathrm{E}_{\mathrm{rs}}$. The final plaquette parameters $K, J$ and $\Delta$ were calibrated independently (Sect. 4.3.2 and 4.4).

\section{Spin state preparation}

Different spin-superposition states were prepared by modifying the final ramp of the filtering sequence (Fig. 6.9 and Sect. 4.5), which was used to remove double occupancies from the trap. Changing the duration of the microwave-pulse (MW-pulse) during the last Landau-Zener sweep (LZ sweep) results in different spin-superposition states $|\psi\rangle_{\text {spin }}=\alpha|\uparrow\rangle+\beta|\downarrow\rangle$. If the MW-pulse is applied during the total time of the $2^{\text {nd }} \mathrm{LZ}$ sweep a fully polarized sample will be obtained with all atoms in the $|\uparrow\rangle$ state, while if the pulse is removed completely during that sweep all atoms will remain in the $|\downarrow\rangle$ state. 


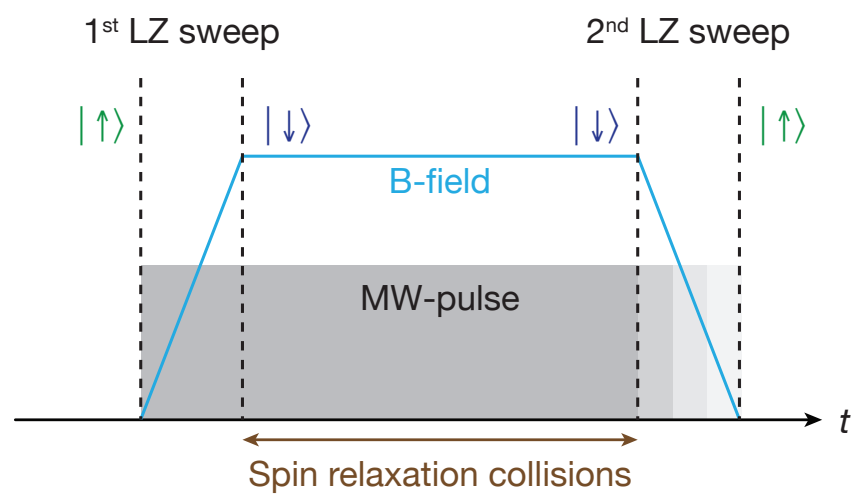

Figure 6.9: Preparation of different spin-superposition states. Schematic drawing of the filtering sequence (Sect. 4.5) used to remove double occupancies from the trap through spin-relaxation collisions. During this sequence the atoms are first transferred to the upper hyperfine-manifold $|\downarrow\rangle=\left|F=2, m_{F}=-1\right\rangle$ using a Landau-Zener (LZ) sweep (magnetic field ramp). After a certain hold time during which atoms on doubly-occupied sites are lost from the trap the atoms are typically transferred back to the $|\uparrow\rangle=\left|F=1, m_{F}=-1\right\rangle$ by applying a $2^{\text {nd }}$ LZ sweep. The gray shaded area illustrates the duration of the MW-pulse driving the hyperfine transition at $\sim 6.8 \mathrm{GHz}$. By changing the duration of the pulse during the $2^{\text {nd }}$ LZ sweep the atoms end up in a spin-superposition state $|\psi\rangle_{\text {spin }}=\alpha|\uparrow\rangle+\beta|\downarrow\rangle$.

Choosing any duration in between those two values will lead to a superposition state $|\psi\rangle_{\text {spin }}$. The probability of finding an atom in a particular spin state was calibrated independently for each pulse duration by releasing the atoms from the trap and applying a Stern-Gerlach pulse at the beginning of TOF. Using usual absorption imaging techniques the fraction of atoms in each spin state: $n_{\uparrow}=|\alpha|^{2}$ and $n_{\downarrow}=|\beta|^{2}$, was evaluated. 


\section{Chapter 7}

\section{All-optical setup for flux rectification}

In the previous chapter a new experimental technique for the realization of uniform artificial gauge fields in optical square lattices was demonstrated. It has been shown that the flux distributions can be probed with local cyclotron orbits $[68,70]$. The same technique further enabled the observation of chiral currents in optical ladder potentials [72]. However, observing the global properties of the system in a full 2D lattice remained out of reach with current setups [70, 71].

The main limitation is rooted in the lack of an adiabatic loading procedure that connects the lattice with trivial topology to the one with non-trivial topology. Starting from a normal square lattice and simply turning on the flux would lead to a splitting of the spectrum into several subbands and the atoms would potentially redistribute over the magnetic subbands in a non-trivial manner. The use of a magnetic field gradient (chapter 6) further limits the experimental flexibility in the design of possible loading procedures.

In this chapter a new experimental setup is introduced which relies solely on fardetuned laser beams and offers the possibility of addressing neighboring bonds in the lattice individually $[66,73]$. In a staggered superlattice potential there are two kinds of bonds with positive and negative energy offset between neighboring sites, which will naturally lead to staggered flux distributions if tunneling is restored on all bonds with the same laser beams (chapter 5). The topology of this system is trivial and the energy bands exhibit a Chern number of zero. With the new scheme tunneling along the two kinds of bonds can be controlled individually. In particular, the sign of the Peierls phases $\phi_{m, n}$ can be adjusted experimentally, which in turn facilitates flux rectification in a square optical lattice with staggered energy offset. In the next chapter (chapter 8) this setup serves as the starting point for an adiabatic loading of the atoms into the Hofstadter bands for $\Phi=\pi / 2$ and it further enabled the first Chern-number measurement in a non-electronic system [73]. 


\subsection{Laser-assisted tunneling on every other bond}

The main ingredient to achieve flux rectification with a staggered superlattice potential is the ability to control tunneling on neighboring bonds in the lattice individually. This is achieved by performing a slight modification to the setup introduced in Sect. 5.3. The new beam configuration is illustrated in Fig. 7.1a. In this setup the running-wave beam along the $x$-direction is retro-reflected, creates a standing wave and interferes with the running wave along $y$. The corresponding electric fields are defined according to

$$
\mathbf{E}_{1}(\mathbf{r}, t)=2 E_{1} \mathrm{e}^{i\left(\omega_{1} t+\phi_{1}\right)} \cos \left(k_{l} x+\varphi\right), \quad \mathbf{E}_{2}(\mathbf{r}, t)=E_{2} \mathrm{e}^{i\left(-k_{l} y+\omega_{2} t+\phi_{2}\right)},
$$

where the phase $\varphi$ determines the position of the modulation relative to the underlying staggered superlattice potential (Sect. 4.3)

$$
V_{S L}(x)=V_{x l} \cos ^{2}\left(k_{l} x\right)+V_{x} \cos ^{2}\left(k_{s} x+\pi / 2\right),
$$

with $k_{l}=k_{s} / 2$ and $k_{s}=2 \pi / \lambda_{s}$. For technical reasons the direction of the laser beam along $y$ (dark red arrow in Fig. 7.1a) was changed compared to the previous setup. The phase $\varphi$ can be adjusted by changing the frequency of the laser beam similar to the phase of the superlattice potential $\varphi_{S L}$ as discussed in Sect. 4.3.

The time-dependent optical potential created by the two beams in Eq. (7.1) can be written in the following form

$$
V(x, y, t)=\underbrace{4 V_{1} \cos ^{2}\left(k_{l} x+\varphi\right)}_{\text {standing wave } V_{\mathrm{sw}}}+\underbrace{V_{2}}_{\text {cst. }}+V_{0} \cos \left(k_{l} x+\varphi\right) \cos \left(-k_{l} y+\omega t+\phi\right),
$$

where $\omega=\omega_{2}-\omega_{1}$ and $\phi=\phi_{2}-\phi_{1}$. The potential amplitudes are proportional to the corresponding field amplitudes $V_{1} \propto E_{1}^{2}, V_{2} \propto E_{2}^{2}$ and $V_{0} \propto 4 E_{1} E_{2}$ [158]. For an appropriate choice of the relative phase $\varphi= \pm \pi / 4$ the modulation induces tunneling only on every other bond. On the remaining bonds the modulation between neighboring sites is in phase (Fig. 7.1b) and does not induce tunneling as discussed in Sect. 3.4. To simplify notations the time-dependent potential $V(x, y, t)$ is written as

$$
V_{m, n}(t)=V_{\mathrm{sw}}+V_{0} \cos (m \pi / 2+\varphi) \cos (-n \pi / 2+\omega t+\phi),
$$

where $\mathbf{R}=m a \hat{\mathbf{e}}_{x}+n a \hat{\mathbf{e}}_{y}$ denotes the position in the lattice. The constant part of the potential was neglected since it only adds a global energy offset.

In the experiment the applicability of this method was tested in isolated double-well potentials, where tunneling on every other bond is suppressed by a high potential barrier (gray bonds in Fig. 7.2a) and all dynamics is restricted to two sites only. Tunneling within each double-well is initially inhibited by a potential energy offset $\Delta$ and is then restored resonantly with $\omega=\Delta / \hbar$ using the laser beam configuration explained above. 
a

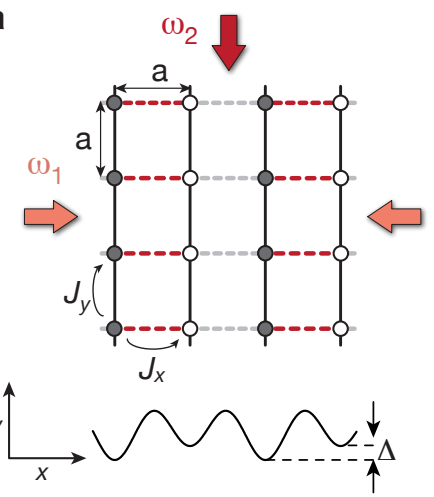

b

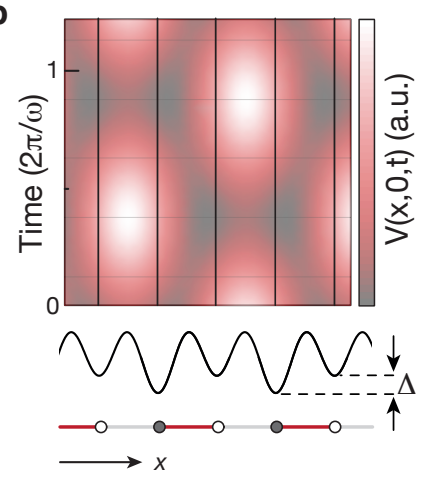

Figure 7.1: Experimental setup for laser-assisted tunneling on every other bond. a Schematic drawing of the experimental setup. Along the $x$-direction of a 2D lattice with lattice constant $a=\lambda_{s} / 2$ a staggered superlattice potential with $\Delta \gg J_{x}$ is used to inhibit tunneling. The bare tunnel couplings are denoted as $J_{x}$ and $J_{y}$. An additional pair of far-detuned laser beams with $\left|\omega_{2}-\omega_{1}\right|=\Delta / \hbar$ and wave vector $k_{l}=\pi /(2 a)$ restores resonant tunneling along that axis in order to create complex tunnel couplings. The pair consists of a running wave along $y$ and a standing wave along $x$. The corresponding optical potential Eq. (7.3) is adjusted such that tunneling is induced on every other bond; for $\varphi=-\pi / 4$ on red bonds and for $\varphi=+\pi / 4$ on gray bonds. $\mathbf{b}$ Time-dependent on-site modulation $V(x, y, t)$ for $y=0$ as a function of time for $\varphi=-\pi / 4$ as defined in Eq. (7.3). Neighboring sites with negative energy offset $-\Delta$ are modulated in phase therefore no tunneling is induced. It is only induced on bonds with positive energy offset $+\Delta$. (Figure adapted from Ref. [73])

The differential modulation amplitude between neighboring sites in the double-well is given by

$$
\delta V=V_{m+1, n}-V_{m, n}=\sqrt{2} V_{0}|\sin (\varphi)|,
$$

which results in an effective tunnel coupling $K=J_{x} \mathcal{J}_{1}(\delta V / \Delta)$. For $\varphi=0$ the long lattice and the on-site modulation are in phase. Hence, the two sites of the doublewell potential are modulated in phase and the relative modulation amplitude vanishes. Ideally, this results in a vanishing effective coupling $K=0$. For $\varphi=\pi / 2$ the relative modulation amplitude reaches its maximum value such that $K=J_{x} \mathcal{J}_{1}\left(\sqrt{2} V_{0} / \Delta\right)$.

The tunnel couplings $K$ were measured for different values of the phase $\varphi$ using a similar sequence as described in Sect. 4.4, where possible doubly occupied sites were emptied by applying a filtering sequence (Sect. 4.5). The final lattice parameters were $V_{x}=7.0(2) \mathrm{E}_{\mathrm{rs}}, V_{x l}=35(1) \mathrm{E}_{\mathrm{rl}}, \varphi_{S L}^{x}=0.073(2) \pi, V_{y}=30(1) \mathrm{E}_{\mathrm{rs}}$ and $V_{z}=30(1) \mathrm{E}_{\mathrm{rz}}$. This corresponds to a tilted double-well potential (Sect. 4.3) with energy offset $\Delta / \hbar \approx 4.5 \mathrm{kHz}$ between the two sites, which was calibrated independently by performing spectroscopy measurements for each value of the phase $\varphi$ (Sect. 4.3.2). Note that due to the additional standing-wave term in Eq. (7.4) the tilted double-well potential is slightly modified. This leads to a dependence of the resonance frequency on the relative phase $\varphi$ on the order 
a

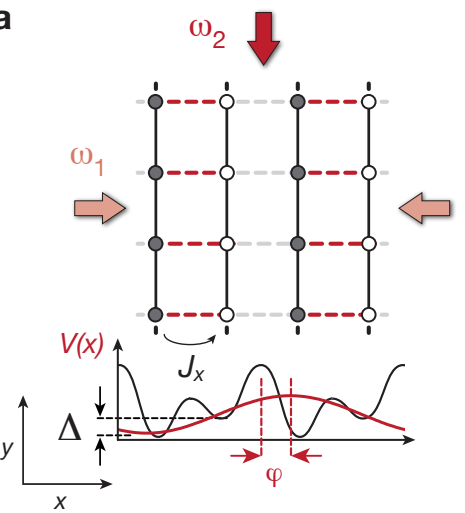

b

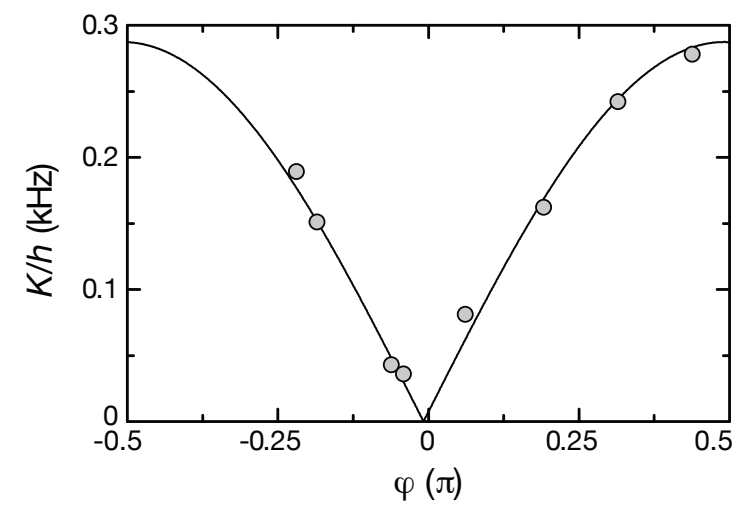

Figure 7.2: Suppression of tunneling on every other bond in isolated double-well potentials. a Schematic drawing of the experimental setup. Along $x$ a tilted double-well potential with energy offset $\Delta \gg J_{x}$ is used to inhibit tunneling; $J_{x}$ is the bare inner-well coupling. Resonant tunneling is then restored using a pair of laser beams (red arrows) that creates a time-dependent potential $V(x, y, t)$ as defined in Eq. (7.4). The potential is illustrated with the red solid line for $y=0$ and $t=0$. Depending on the relative phase $\varphi$, the effective tunnel coupling $K$ varies between zero and $K=J_{x} \mathcal{J}_{1}\left(\sqrt{2} V_{0} / \Delta\right)$. b Measured effective coupling strength as a function of the phase $\varphi$. The solid line is a fit to our data using $f(\varphi)=A_{0}\left|\sin \left(\varphi+\varphi_{0}\right)\right|$, where the amplitude $A_{0}=0.287(6) \mathrm{kHz}$ and the phase offset $\varphi_{0}=0.008(5) \pi$ were free fit variables. (Figure adapted from Ref. [73])

of $20 \%$.

Using the measured coupling strengths displayed in Fig. 7.2b an upper limit for the suppression of tunneling on every other bond was obtained, $K_{\min } / K_{\max }<0.13$. A residual non-zero coupling obtained when the modulation between neighboring sites is in phase, is most likely due to an imperfect back-reflection of the running-wave beam along $x$ (light red arrow in Fig. 7.2a).

\subsection{Uniform flux in a staggered potential}

With the experimental setup described in the previous section tunneling could be restored on every other bond (red bonds in Fig. 7.1a). This is the starting point for the flux-rectification scheme. In the following two pairs of beams are applied in the same configuration as described above (red and blue arrows in Fig. 7.3a)

$$
V_{m, n}^{i}(t)=V_{\mathrm{sw}}^{i}+V_{0}^{i} \cos \left(m \pi / 2+\varphi_{i}\right) \cos \left(-n \pi / 2+\omega_{i} t+\phi_{i}\right),
$$

where $\omega_{i}=\omega_{i 2}-\omega_{i 1}, i=\{r, b\}$. For $\varphi_{r}=-\pi / 4$ and $\varphi_{b}=\pi / 4$ two independent on-site modulation potentials are obtained (Fig. $7.3 \mathrm{~b}, \mathrm{c}$ ), which are spatially separated by one lattice constant, so that the two kinds of bonds in the lattice (red and blue) can be addressed individually. As a side effect the two standing-wave terms $V_{\mathrm{SW}}^{i}$ cancel each 

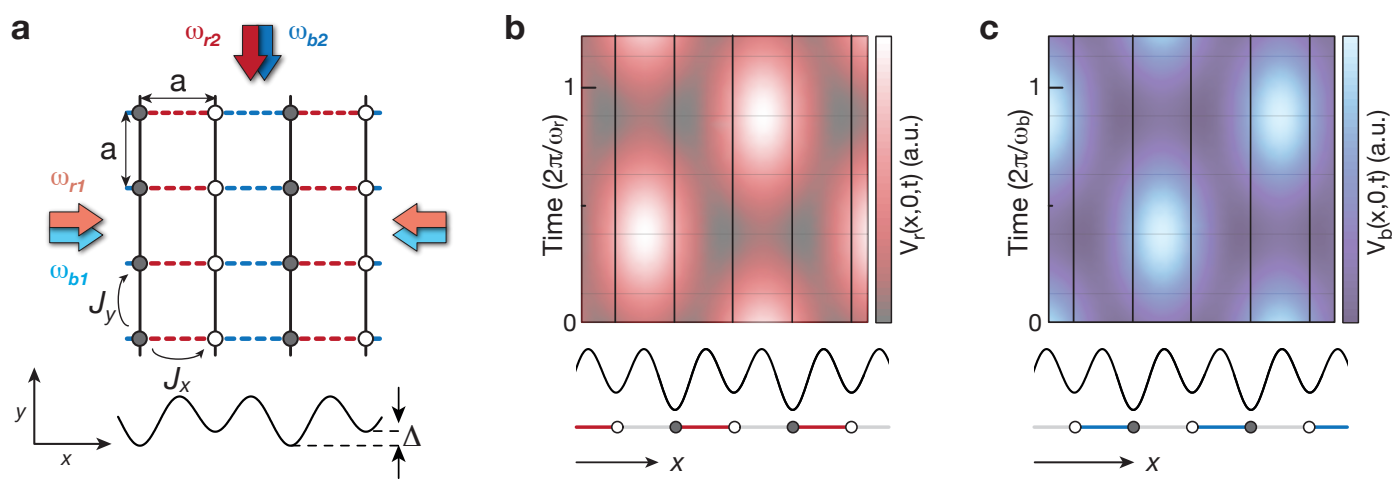

Figure 7.3: All-optical flux-rectification scheme for laser-assisted tunneling in a staggered superlattice potential. a Schematics of the all-optical setup. A staggered superlattice potential with offset $\Delta \gg J_{x}$ inhibits tunneling between neighboring sites (gray and white). Two additional pairs of beams (red and blue arrows) restore resonant tunneling with $\omega_{i}=\omega_{i 2}-\omega_{i 1}= \pm \Delta / \hbar$ and wave vectors $\left|\mathbf{k}_{i j}\right| \simeq k_{l}=\pi /(2 a)$, with $i=\{r, b\}$ and $j=\{1,2\}$. Each pair is composed of one running wave along $y$ and one standing wave along $x$ (arrows with lighter shading). Tunneling on different bonds with opposite sign of the energy offset $\pm \Delta$ (red and blue) is controlled independently with the red and blue beams, which results in a uniform flux distribution $\Phi=\pi / 2$ per plaquette (aligned along - $\left.\hat{\mathbf{e}}_{z}\right)$. $\mathbf{b}, \mathbf{c}$ Total time-dependent potentials $V_{i}(x, 0, t)$ as a function of time for the red (b) and blue (c) beams. The differential modulation amplitude between neighboring sites $V_{m+1, n}^{i}-V_{m, n}^{i}$ vanishes on every other bond (gray bonds) and tunneling is restored on red bonds (b) and blue bonds (c) independently. (Figure adapted from Ref. [73])

other. The modulation amplitudes $V_{0}^{r}=V_{0}^{b} \equiv V_{0}$ are the same for both pairs by construction as will be demonstrated in Sect. 7.3.

The system can be described using the following time-dependent tight-binding Hamiltonian

$$
\begin{aligned}
\hat{H}(t)= & \sum_{m, n}\left(-J_{x} \hat{a}_{m+1, n}^{+} \hat{a}_{m, n}-J_{y} \hat{a}_{m, n+1}^{+} \hat{a}_{m, n}+\text { h.c. }\right)+\sum_{m, n}(-1)^{m} \frac{\Delta}{2} \hat{n}_{m, n} \\
& +\sum_{m, n}\left[V_{0} \cos \left(m \frac{\pi}{2}-\frac{\pi}{4}\right) \cos \left(-n \frac{\pi}{2}+\omega_{r} t+\phi_{r}\right)\right] \hat{n}_{m, n} \\
& +\sum_{m, n}\left[V_{0} \cos \left(m \frac{\pi}{2}+\frac{\pi}{4}\right) \cos \left(-n \frac{\pi}{2}+\omega_{b} t+\phi_{b}\right)\right] \hat{n}_{m, n} .
\end{aligned}
$$

In the high-frequency limit $\hbar \omega \gg J_{x}, J_{y}$, where $\omega:=\left|\omega_{r}\right|=\left|\omega_{b}\right|=\Delta / \hbar$, the corresponding effective Floquet Hamiltonian can be derived in the rotating frame as discussed in Sect. 3.4. The unitary transformation is defined in Eq. (3.73) with

$$
\begin{aligned}
\chi_{m, n}(t) & =\frac{V_{0}}{\hbar \omega_{r}} \cos \left(m \frac{\pi}{2}-\frac{\pi}{4}\right) \sin \left(-n \frac{\pi}{2}+\omega_{r} t+\phi_{r}\right) \\
& +\frac{V_{0}}{\hbar \omega_{b}} \cos \left(m \frac{\pi}{2}+\frac{\pi}{4}\right) \sin \left(-n \frac{\pi}{2}+\omega_{b} t+\phi_{b}\right)+(-1)^{m} \frac{\Delta t}{2 \hbar} .
\end{aligned}
$$


The transformed Hamiltonian $\hat{\mathcal{H}}(t)$ defined in Eq. (3.74) involves time-dependent complex tunnel couplings with phase factors $\eta_{m, n}^{x}(t)=\chi_{m+1, n}(t)-\chi_{m, n}(t)$ for hopping along the $x$-direction. For the experimental setup these phases can be expressed in the following form

$$
\eta_{m, n}^{x}(t)= \begin{cases}-\frac{\sqrt{2} V_{0}}{\hbar \omega_{r}} \sin \left(-n \frac{\pi}{2}+\omega_{r} t+\phi_{r}-(m-1) \frac{\pi}{2}\right)+\frac{\Delta t}{\hbar} & \text { for } m \text { odd } \\ -\frac{\sqrt{2} V_{0}}{\hbar \omega_{b}} \sin \left(-n \frac{\pi}{2}+\omega_{b} t+\phi_{b}-m \frac{\pi}{2}\right)-\frac{\Delta t}{\hbar} & \text { for } m \text { even }\end{cases}
$$

Taking the time average over one driving period $T=2 \pi / \omega$ according to Eq. (3.35) results in the effective time-independent Hamiltonian given by

$$
\hat{\mathcal{H}}_{\mathrm{F}}=\sum_{m, n}\left(-\mathrm{Ke}^{i \phi_{m, n}} \hat{a}_{m+1, n}^{\dagger} \hat{a}_{m, n}-J \hat{a}_{m, n+1}^{\dagger} \hat{a}_{m, n}+\text { h.c. }\right),
$$

where $K=J_{x} \mathcal{J}_{1}\left(\sqrt{2} V_{0} / \Delta\right)$. For $\omega_{r}=-\omega_{b}= \pm \Delta / \hbar$ the corresponding Peierls phasefactors are determined by

$$
\phi_{m, n}= \begin{cases} \pm \frac{\pi}{2}(m+n-1) \mp \phi_{r}, & \text { for } m \text { odd } \\ \pm \frac{\pi}{2}(m+n) \mp \phi_{b}, & \text { for } m \text { even }\end{cases}
$$

which gives rise to a uniform flux distribution with $\Phi=\phi_{m, n+1}-\phi_{m, n}= \pm \pi / 2$ per plaquette. Note that by choosing $\omega_{r}=\omega_{b}= \pm \Delta / \hbar$ a staggered flux distribution is obtained (Sect. 7.4).

In order to derive the effective coupling strength $J$ perpendicular to the complex tunneling direction the time-dependent phases-factors $\eta_{m, n}^{y}(t)=\chi_{m, n+1}(t)-\chi_{m, n}(t)$ have to be considered

$$
\begin{aligned}
\eta_{m, n}^{y}(t) & =-\frac{\sqrt{2} V_{0}}{\hbar \omega_{r}} \cos \left(m \frac{\pi}{2}-\frac{\pi}{4}\right) \cos \left(-n \frac{\pi}{2}+\omega_{r} t+\phi_{r}-\frac{\pi}{4}\right) \\
& -\frac{\sqrt{2} V_{0}}{\hbar \omega_{b}} \cos \left(m \frac{\pi}{2}+\frac{\pi}{4}\right) \cos \left(-n \frac{\pi}{2}+\omega_{b} t+\phi_{b}-\frac{\pi}{4}\right) .
\end{aligned}
$$

In contrast to the restored tunneling along the $x$-axis, tunneling along $y$ is affected by both modulation potentials simultaneously for each bond and the differential modulation amplitude between neighboring sites cannot be evaluated by treating the two modulations separately.

The combined on-site modulation is characterized by two distinct phases, i.e. the relative phase of the modulation $\delta \phi=\phi_{r}-\phi_{b}$ and the initial phase of the modulation $\phi_{0}:=\phi_{r}$. The relative phase $\delta \phi$ can be controlled experimentally as will be shown in Sect. 7.3.1 but the initial phase of the driving is not stabilized. For the sake of simplicity the relative phase is set to $\delta \phi=-\pi / 2$ for the following discussion. Together with $\omega:=\omega_{r}=-\omega_{b}$ the equations above simplify to 
a

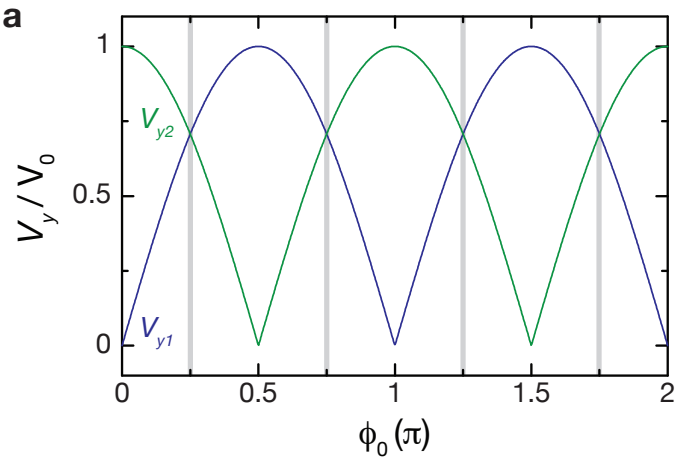

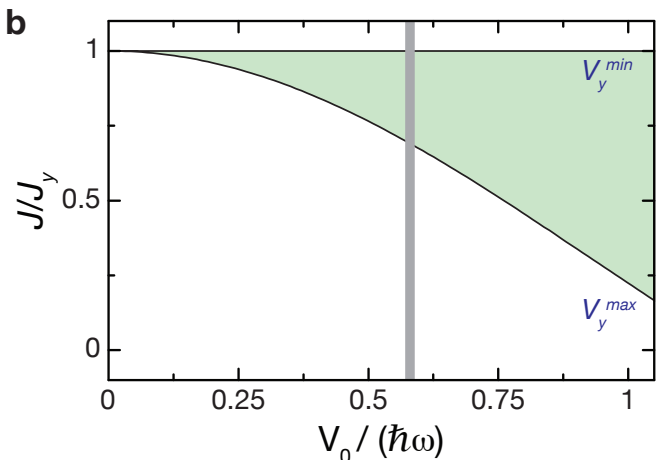

Figure 7.4: Relative modulation amplitudes $V_{y}$ and effective coupling strengths $J$ along the $y$ direction. a The lattice modulation (red and blue arrows in Fig. 7.3b) leads to a renormalization of the tunneling along $y$. The differential modulation amplitude between neighboring sites $V_{y}$ depends on the initial phase $\phi_{0}$ and is potentially inhomogeneous over the 2D lattice determined by the two values $V_{y 1}$ and $V_{y 2}$ (green and purple). For a special choice of the phase $\phi_{0}=$ $(v \pm 1 / 4) \pi, v \in \mathbb{Z}$, the differential modulation amplitude is homogeneous (gray vertical lines). $\mathbf{b}$ Possible values of the effective coupling $J$ for different phases $\phi_{0}$ as a function of the modulation amplitude $V_{0}$. The minimum value of $J$ is determined by the maximum modulation amplitude $V_{y}^{\max }=V_{0}$ and the maximum value is equal to the bare tunnel coupling $J_{y}$ for $V_{y}^{\min }=0$. The inhomogeneity in the coupling strength increases with increasing modulation amplitude $V_{0}$. The gray vertical line marks the value $V_{0} /(\hbar \omega) \approx 0.58$ used for the experiments presented in the next chapter (chapter 8), for which the couplings vary between $J_{\max }=J_{y}$ and $J_{\min }=0.7 J_{y}$.

$$
\eta_{m, n}^{y}(t)= \pm \frac{2 V_{0}}{\hbar \omega} \begin{cases}\cos \left(\phi_{0}\right) \sin \left(\omega t \pm \frac{\pi}{4}\right), & \text { for }(m+n) \text { odd } \\ \sin \left(\phi_{0}\right) \sin \left(\omega t \pm \frac{\pi}{4}\right), & \text { for }(m+n) \text { even }\end{cases}
$$

Along $y$ the effective tunnel coupling is renormalized according to the zeroth-order Bessel function of the first kind $J=J_{y} \mathcal{J}_{0}\left(2 V_{y} / \Delta\right)$ but it does not involve any Peierls phase-factors (Sect. 3.2 and 3.4). Therefore only the oscillation amplitude of $\eta_{m, n}^{y}(t)$ is of interest but not its phase. According to the Eq. (7.13) the amplitude can take two different values $V_{y 1}=V_{0} \sin \left(\phi_{0}\right)$ and $V_{y 2}=V_{0} \cos \left(\phi_{0}\right)$ depending on $\phi_{0}$ (Fig. 7.4a). Intuitively, this phase determines the position of the modulation relative to the underlying lattice along $y$. For each experimental realization a different value of the phase $\phi_{0}$ is obtained, which potentially leads to inhomogeneous couplings along the $y$-direction. However, in the limit $V_{0} /(\hbar \omega) \ll 1$, the effective coupling can be very well approximated by the bare tunnel coupling $J \simeq J_{y}$, such that it is approximately homogeneous over the 2D lattice (Fig. 7.4b).

Note that stabilizing the value to $\phi_{0}=(v \pm 1 / 4) \pi, v \in \mathbb{Z}$, would result in homogeneous couplings independent of the driving amplitude $V_{0}$ (gray vertical lines in Fig. 7.4a) and the corresponding effective coupling would be given by $J=J_{y} \mathcal{J}_{0}\left(\sqrt{2} V_{0} / \Delta\right)$.

If this is not the case and $V_{0}$ is on the order of the driving frequency than the effec- 
tive couplings $J$ can be approximated up to second order in $V_{0} /(\hbar \omega)$ using the series expansion of the zeroth order Bessel function of the first kind $\mathcal{J}_{0}(x) \approx 1-x^{2} / 4+\mathcal{O}\left(x^{4}\right)$

$$
J=J_{y} \begin{cases}\mathcal{J}_{0}\left(\frac{2 V_{0}}{\hbar \omega} \cos \left(\phi_{0}\right)\right) \approx 1-\frac{V_{0}^{2}}{2(\hbar \omega)^{2}}\left(1+\cos \left(2 \phi_{0}\right)\right), & \text { for }(m+n) \text { odd } \\ \mathcal{J}_{0}\left(\frac{2 V_{0}}{\hbar \omega} \sin \left(\phi_{0}\right)\right) \approx 1-\frac{V_{0}^{2}}{2(\hbar \omega)^{2}}\left(1-\cos \left(2 \phi_{0}\right)\right), & \text { for }(m+n) \text { even }\end{cases}
$$

Finally this expressions can be combined into a single equation

$$
J \simeq J_{y}\left(1+f_{m, n}\right), \quad f_{m, n}=-\frac{1}{2}\left(\frac{V_{0}}{\hbar \omega}\right)^{2}\left[1-(-1)^{m+n} \cos \left(2 \phi_{0}\right)\right] .
$$

An equivalent derivation of the effective Hamiltonian using the formalism discussed in Sect. 3.1.2 can be found in Refs. [66, 73].

\subsection{Experimental setup}

The two pairs of beams (red and blue arrows in Fig. 7.3a) are generated using a single laser $^{1}$ (Fig. 7.5a) with wavelength $\lambda_{l}=1534 \mathrm{~nm}$, which is locked to the short lattice $\lambda_{s}=767 \mathrm{~nm}$ via a frequency offset lock. This is similar to the frequency control of the two long lattices which participate in the generation of the bichromatic superlattice potentials (Sect. 4.3). The laser beam is first split into two parts, each of them is passing through an acousto-optic modulator (AOM) with frequency $\omega_{A}=2 \pi \times 80 \mathrm{MHz}$ in order to stabilize the intensity of each beam individually with a feedback loop [162]. Then each beam is sent through a fiber coupled intensity modulator ${ }^{2}$ (IM) to generate sidebands at frequencies $\pm \Omega_{j}, j=\{1,2\}$. At the same time the carrier frequency at $\omega_{c}+\omega_{A}$, where $\omega_{c}$ denotes the laser frequency, is suppressed by the IM. By choosing $\Omega_{2}-\Omega_{1}=$ $\pm \Delta / \hbar$ the condition required for the uniform flux configuration $\omega_{r}=-\omega_{b}= \pm \Delta / \hbar$ is automatically fulfilled (Fig. 7.5b).

Relative position between the modulation and the superlattice potential: For the local addressing to work the on-site modulation has to be further adjusted spatially, relative to the underlying superlattice potential, which consists of a short and a long lattice. The relative position is determined by the phases $\varphi_{r}$ and $\varphi_{b}$ respectively. Analog to the phase of the superlattice potential the standing wave along $x$ (light arrows in Fig. 7.3) accumulates a phase until it reaches the atom position (Fig. 4.3), hence, it can be adjusted by controlling its frequency relative to the one associated with the short lattice using a frequency offset lock (Sect. 4.3).

\footnotetext{
${ }^{1}$ Erbium doped fiber amplifier from NP Photonics (output power: $5 \mathrm{~W}$ )

${ }^{2}$ EOSPACE Lithium Niobate Modulator AX-0K5-00-PFA-PFA-NT-UL
} 

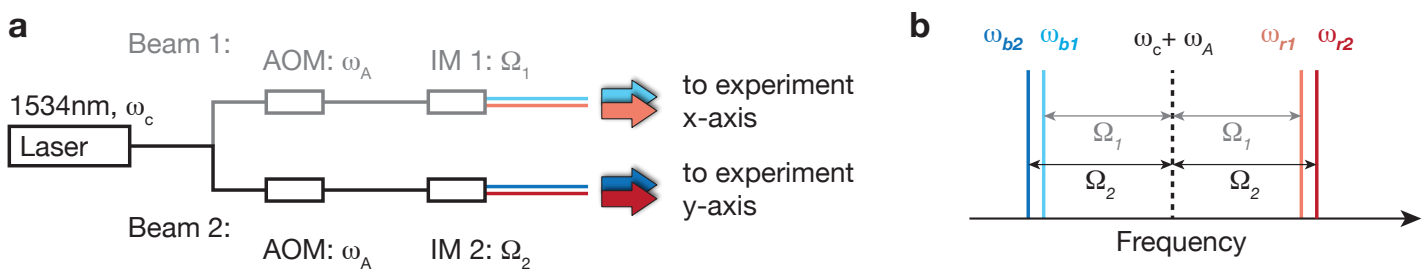

Figure 7.5: Schematics of the experimental setup for flux rectification. a A fiber amplifier with frequency $\omega_{c}$ is used to generate all four beams for the modulation scheme illustrated in Fig. 7.3a. The main beam is split into two parts: Beam 1 and 2 (gray and black solid line). Each of them passes through an AOM with frequency $\omega_{A}$ and a fiber coupled IM that creates sidebands at $\pm \Omega_{j}, j=\{1,2\}$ and suppresses the carrier frequency at $\omega_{c}+\omega_{A}$. Thus, Beam 1 is split into two beams with frequencies $\omega_{r 1, b 1}=\omega_{c}+\omega_{A} \pm \Omega_{1}$ (light red and light blue) and realizes the standing waves along $x$. Beam 2 is the source for the two running waves along $y$ with frequencies $\omega_{r 2, b 2}=\omega_{c}+\omega_{A} \pm \Omega_{2}$ (dark red and dark blue). $\mathbf{b}$ Illustration of the different frequencies generated in the experimental setup. The dashed black line indicates that the carrier frequency at $\omega_{c}+\omega_{A}$ is suppressed after the IM. The solid lines (red and blue) indicate the frequencies $\omega_{i, j}, i=\{r, b\}$, which are used for the modulation. Note that the distances are not to scale, $2 \Omega_{1,2} \gg\left|\Omega_{2}-\Omega_{1}\right|$ so that inference between the sidebands (red and blue beams) can be neglected.

Alignment procedure: First, the phase $\varphi$ is calibrated for $\Omega_{j}=0$ by creating a bichromatic superlattice potential which consists of the short lattice and the standing wave along $x$ (Beam 1 in Fig. 7.5a). The relevant techniques are described in Sect. 4.3. This will determine the position of the modulation relative to the superlattice potential. In addition the phase between the long lattice and the standing-wave along $x$ (Beam 1) can be inferred by applying both potentials simultaneously $\left(\Omega_{j}=0\right)$. If the two beams are in phase, the corresponding potentials will add up, but if they are out of phase, they will cancel each other.

As a second step the frequencies of the sidebands $\pm \Omega_{j}$ need to be adjusted. The frequency difference $\delta v_{a}$ required to shift the long-lattice standing wave by one lattice constant $a=\lambda_{l} / 4$ is known from the calibration measurements depicted in Fig. 4.4, $\delta v_{a}=740(10) \mathrm{MHz}=2 \delta v_{l}$, with $v_{l}=c / \lambda_{l}$. By choosing the frequencies $2 \Omega_{1} \simeq 2 \Omega_{2}=$ $2 \pi \times 370(5) \mathrm{MHz}$ for the generation of the sidebands a relative shift of one lattice constant between the two modulations is ensured, which corresponds to $\varphi_{b}-\varphi_{r}=\pi / 2$. All relevant phase relations between the superlattice potential and the lattice-modulation beams are very stable by construction since the phases are determined by the position of the mirror, which is used to retro-reflect all beams.

Interference between the four frequency components: The interference terms between the sidebands at $\pm \Omega_{j}$ can be safely neglected since the two timescales are well separated $2 \Omega_{j} /\left|\Omega_{2}-\Omega_{1}\right|=2 \Omega_{j} / \Delta \simeq 10^{5}$. Only interference of the laser beams along $x$ 
with the ones along $y$ are considered in the following description. Since the sidebands are created symmetrically using intensity modulation, the corresponding amplitudes are equal, $\left|E_{r 1}\right|=\left|E_{b 1}\right|$ and $\left|E_{r 2}\right|=\left|E_{b 2}\right|$, which in turn results in equal modulation amplitudes of the two pairs, $V_{0}^{r} \propto E_{r 1} E_{r 2}, V_{0}^{b} \propto E_{b 1} E_{b 2}$ and $V_{0}^{r}=V_{0}^{b}=V_{0}$.

\subsubsection{Intensity modulation}

The fiber coupled intensity modulator consists of two interferometer arms (Fig. 7.6). In each arm there is a Pockels cell to modulate the phase of the incoming wave. It is built such that an externally applied voltage is connected to both Pockels cells with opposite polarity, which results in phase modulations with opposite sign for the two interferometer arms $\pm \mathcal{V}_{M} \sin \left(\Omega t+\phi_{I M}\right) \pm \mathcal{V}_{\text {bias }}$. Note that $\mathcal{V}_{M}$ and $\mathcal{V}_{\text {bias }}$ are dimensionless quantities. Let us consider the spectrum at the output of the intensity modulator for an incoming wave $E_{0} \exp (i \omega t)$. The incoming wave is split into equal parts at the beginning of the interferometer $E_{r, b}=E_{0} \exp (i \omega t) / 2$. After the Pockels cells the field is modified according to

$$
E_{r, b}(t)=\frac{E_{0}}{2} \exp \left[i\left(\omega t \pm \mathcal{V}_{M} \sin \left(\Omega t+\phi_{I M}\right) \pm \mathcal{V}_{\text {bias }}\right)\right]
$$

Without modulation $\left(\mathcal{V}_{M}=0\right)$ the modulator can be used as a simple switch. The bias voltage introduces a constant phase shift $\mathcal{V}_{\text {bias }}$ between the two arms of the interferometer which allows one to adjust the transmitted intensity $I_{\text {out }}$ ideally between zero and $I_{0}=\left|E_{0}\right|^{2}$ if additional losses are neglected, such that $I_{\text {out }}=I_{0} \cos ^{2}\left(\mathcal{V}_{\text {bias }}\right)$, see Fig. 7.7a. For the device used in the experiment the maximum transmission is typically limited to about $0.5 \mathrm{I}_{0}$ because of the specified insertion loss of $\sim 3 \mathrm{dBm}$. For non-zero modulation amplitude $\left(\mathcal{V}_{M} \neq 0\right)$ the spectrum contains multiple frequency components at $\omega+k \Omega$, $k \in \mathbb{Z}$. This can be seen by rewriting Eq. (7.16) in terms of the Bessel functions of the first kind

$$
\begin{aligned}
E_{r, b}(t)=\frac{E_{0}}{2} \mathrm{e}^{i\left(\omega t \pm \mathcal{V}_{\text {bias }}\right)} & {\left[\mathcal{J}_{0}\left( \pm \mathcal{V}_{M}\right)+\sum_{k=1}^{\infty} \mathcal{J}_{k}\left( \pm \mathcal{V}_{M}\right) \mathrm{e}^{i k\left(\Omega t+\phi_{I M}\right)}\right.} \\
& \left.+\sum_{k=1}^{\infty}(-1)^{k} \mathcal{J}_{k}\left( \pm \mathcal{V}_{M}\right) \mathrm{e}^{-i k\left(\Omega t+\phi_{I M}\right)}\right] .
\end{aligned}
$$

The interference between both arms at the output of the IM exhibits the following spectral components 

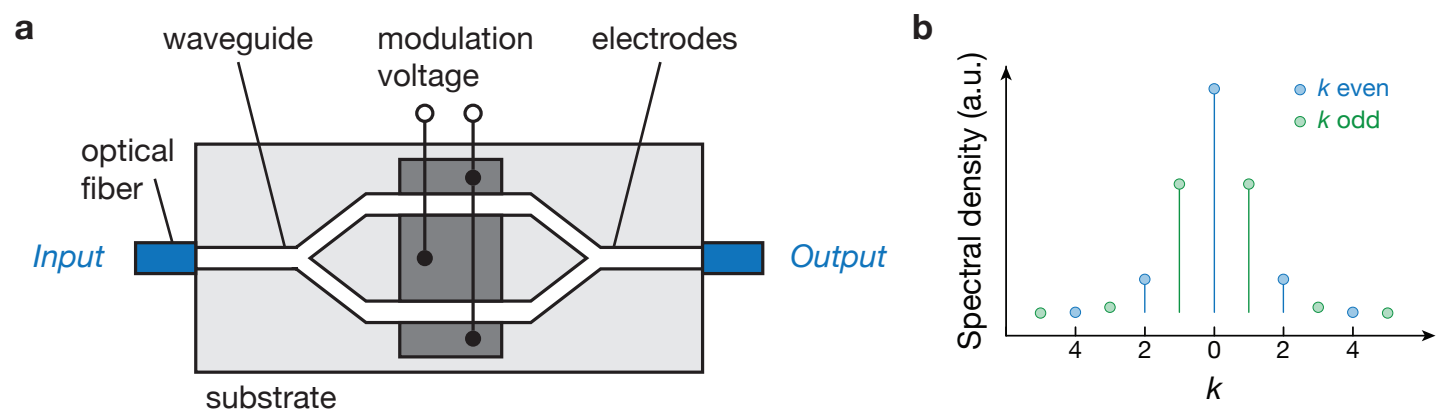

Figure 7.6: Schematic drawing of the intensity modulator that generates the sidebands for the laser-assisted tunneling setup. a The intensity modulator is a Mach-Zehnder interferometer with integrated phase modulators. The input and output optical fibers are polarizationmaintaining. By applying a constant voltage (bias voltage) to the electrodes without modulation $\left(\mathcal{V}_{M}=0\right)$ the relative phase $\mathcal{V}_{\text {bias }}$ between the two interferometer arms is modified and the transmission at the output of the device can be tuned as shown in Fig. 7.7a. By applying a time-dependent voltage to the electrodes $\left(\mathcal{V}_{M}(t)\right)$, the spectrum exhibits additional frequency components at $\omega+k \Omega, k \in \mathbb{Z}$. The spectral density of the individual components depends on the modulation amplitude and offset. (Figure adapted from Jenoptik [188]) $\mathbf{b}$ Illustration of the spectrum at the output of the device for $\mathcal{V}_{\text {bias }}=\pi / 4$ and $\mathcal{V}_{M}=1$ as defined in Eq. (7.18).

$$
\begin{aligned}
E_{\text {out }}=E_{r}(t)+E_{b}(t)=E_{0} \mathrm{e}^{i \omega t} & {\left[\sum_{2 k=-\infty}^{\infty} \mathcal{J}_{k}\left(\mathcal{V}_{M}\right) \mathrm{e}^{i k\left(\Omega t+\phi_{I M}\right)} \cos \left(\mathcal{V}_{\text {bias }}\right)\right.} \\
& +i \sum_{2 k-1=1}^{\infty} \mathcal{J}_{k}\left(\mathcal{V}_{M}\right) \mathrm{e}^{i k\left(\Omega t+\phi_{I M}\right)} \sin \left(\mathcal{V}_{\text {bias }}\right) \\
& \left.-i \sum_{2 k-1=1}^{\infty} \mathcal{J}_{k}\left(\mathcal{V}_{M}\right) \mathrm{e}^{-i k\left(\Omega t+\phi_{I M}\right)} \sin \left(\mathcal{V}_{\text {bias }}\right)\right]
\end{aligned}
$$

Depending on the bias voltage, that determines the phase offset $\mathcal{V}_{\text {bias, }}$, the spectrum either consists of all frequency components $\omega+k \Omega$ (Fig. 7.6b) or only even and odd ones respectively (Fig. 7.7b). The phase $\mathcal{V}_{\text {bias }}$ can be calibrated by measuring the transmitted intensity $I_{\text {out }}=\left|E_{\text {out }}\right|^{2}$ as a function of the bias voltage as shown in Fig. 7.7a. Using this knowledge together with Eq. (7.18) determines the spectral density of the transmitted light $E_{\text {out }}$.

For $\mathcal{V}_{\text {bias }}=\pi / 2$ the carrier frequency is fully suppressed and the spectrum consists of odd frequency components only. Consequently, the spectrum of the output intensity $I_{\text {out}}$, which was measured in the experiment (Fig. 7.7b), contains only spectral components at multiples of $2 \Omega$. The same is true for $\mathcal{V}_{\text {bias }}=0$ even though the spectral decomposition of the transmitted field $E_{\text {out }}$ is very different (Fig. 7.7b). As an example the spectral weight of the frequency component at $2 \Omega$ of the transmitted intensity $I_{\text {out }}$ is computed 

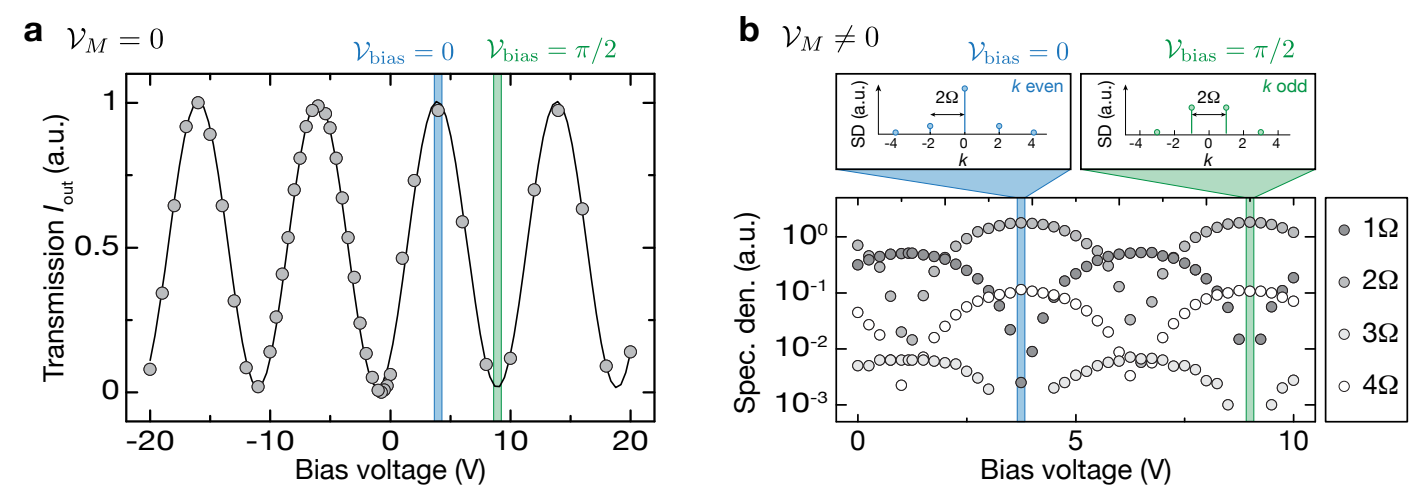

Figure 7.7: Intensity and spectrum of the light at the output of the intensity modulator as a function of the bias voltage. a Measured transmitted intensity $I_{\text {out }}$ versus bias voltage without modulation $\left(\mathcal{V}_{M}=0\right)$. The data depicted in (a) was normalized to its minimum (green vertical lines, $\mathcal{V}_{\text {bias }}=\pi / 2$ ) and maximum (blue vertical line, $\mathcal{V}_{\text {bias }}=0$ ) value. The solid line is a fit of a cosine to our data. For this device a phase shift of $\pi$ corresponds to 9.96(2) V. b Spectrum of the transmitted intensity $I_{\text {out }}=\left|E_{\text {out }}\right|^{2}$ at the output of the IM for $\mathcal{V}_{M} \approx 0.45$ as a function of the bias voltage. The upper panels depict the spectral density of the corresponding spectral decomposition of the transmitted field $E_{\text {out }}$. For $\mathcal{V}_{\text {bias }}=0$ the spectrum consists of even components (blue), while for $\mathcal{V}_{\text {bias }}=\pi / 2$ it consists of odd ones (green). These two spectra cannot be distinguished by measuring the spectrum of the transmitted intensity $I_{\text {out }}$, but in combination with (a) they can be reconstructed according to Eq. (7.18). For $\mathcal{V}_{\text {bias }}=\pi / 4$ the spectrum consists of even as well as odd frequency components as shown in Fig. 7.6b.

$$
I_{2 \Omega}\left(\mathcal{V}_{M}\right)= \begin{cases}2 \sum_{k=0}^{\infty} \mathcal{J}_{2 k}\left(\mathcal{V}_{M}\right) \mathcal{J}_{2 k+2}\left(\mathcal{V}_{M}\right) & \text { for } \mathcal{V}_{\text {bias }}=0 \\ \mathcal{J}_{1}\left(\mathcal{V}_{M}\right)^{2}-2 \sum_{k=1}^{\infty} \mathcal{J}_{2 k-1}\left(\mathcal{V}_{M}\right) \mathcal{J}_{2 k+1}\left(\mathcal{V}_{M}\right) & \text { for } \mathcal{V}_{\text {bias }}=\pi / 2\end{cases}
$$

One can show that both infinite series converge to the same value $\mathcal{J}_{2}\left(2 \mathcal{V}_{M}\right) / 2$.

For $\mathcal{V}_{\text {bias }}=\pi / 4$ the spectrum of the transmitted light $E_{\text {out }}$ contains all even and odd frequency components but the Fourier transform of $I_{\text {out }}$ only consists of components at odd multiples of $\Omega$. Again one may compute the spectral weight of the frequency component at $2 \Omega$

$$
I_{2 \Omega}\left(\mathcal{V}_{M}\right)=\mathcal{J}_{1}\left(\mathcal{V}_{M}\right)^{2}-2 \sum_{k=0}^{\infty} \mathcal{J}_{k}\left(\mathcal{V}_{M}\right) \mathcal{J}_{k+2}\left(\mathcal{V}_{M}\right)=\frac{\mathcal{J}_{2}\left(2 \mathcal{V}_{M}\right)}{2}-\frac{\mathcal{J}_{2}\left(2 \mathcal{V}_{M}\right)}{2}=0
$$

It is determined by the difference between the two infinite series given in Eq. (7.19).

For our experiments the phase offset between the two interferometer arms was set to $\mathcal{V}_{\text {bias }}=\pi / 2$, where the carrier frequency is fully suppressed (Fig. 7.8). The maximum intensity for the two sidebands at $\pm \Omega$ is obtained for $\mathcal{V}_{M}=1.8$ and corresponds to $\sim 0.34 I_{0}$, where $I_{0}$ is the maximum transmitted intensity, which is possibly reduced 
a

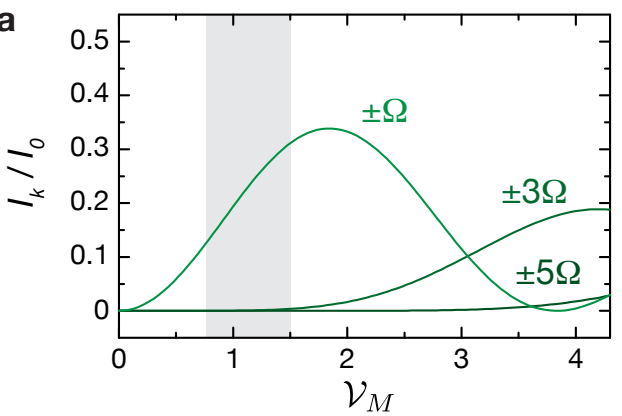

b

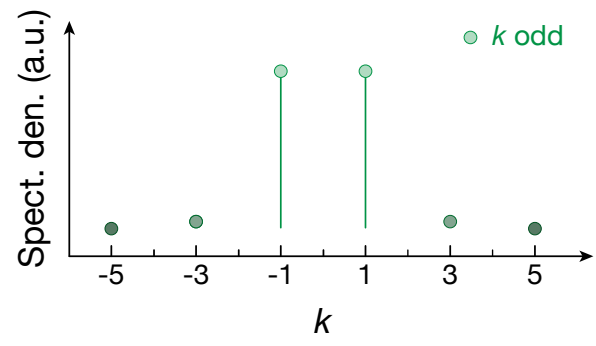

Figure 7.8: Spectral composition of the transmitted light $I_{\text {out }}$ for $\mathcal{V}_{\text {bias }}=\pi / 2$ as a function of the modulation amplitude $\mathcal{V}_{M}$. a Intensity $I_{k}=I_{0}\left(\mathcal{J}_{k}\left(\mathcal{V}_{M}\right)\right)^{2}$ of the odd frequency components $k=\{ \pm 1, \pm 3, \pm 5\}$ as a function of $\mathcal{V}_{M}$. All even frequencies as well as the carrier frequency are suppressed for $\mathcal{V}_{\text {bias }}=\pi / 2$. The gray shaded area marks the regime typically used in the experiment. $\mathbf{b}$ Spectrum for $\mathcal{V}_{\text {bias }}=\pi / 2$ and $\mathcal{V}_{M}=1$

due to the insertion loss at the input of the IM. For the measurements presented in this chapter and the following one (chapter 8) the modulation amplitudes were chosen such that the phase modulation was well below $\mathcal{V}_{M}=\pi / 2$. This sets an upper limit for the suppression of higher frequency components $I_{3} / I_{1}<0.015$, where $I_{k}=I_{0}\left(\mathcal{J}_{k}\left(\mathcal{V}_{M}\right)\right)^{2}$. Consequently, all frequency components with $|k|>2$ can be neglected and the transmitted field can be written as

$$
E_{r}(t)+E_{b}(t) \simeq i E_{0} \mathrm{e}^{i \omega t}[\underbrace{\mathcal{J}_{1}\left(\mathcal{V}_{M}\right) \mathrm{e}^{i\left(\Omega t+\phi_{I M}\right)}}_{\rightarrow \text { red beam }}-\underbrace{\mathcal{J}_{1}\left(\mathcal{V}_{M}\right) \mathrm{e}^{-i\left(\Omega t+\phi_{I M}\right)}}_{\rightarrow \text { blue beam }}] .
$$

To generate the four beams illustrated as blue and red arrows in Fig. 7.3a two intensity modulators are used in the experimental setup (Fig. 7.5), one for each axes. The beam sent along the $x$-axis (Beam 1) is characterized by the electric field $E_{1}(t)=E_{r 1}(t)+E_{b 1}(t)$ and the one along $y$ (Beam 2) is given by an analog expression. Using relation (7.21) the phase between the two modulations $\delta \phi=\phi_{r}-\phi_{b}$ can be inferred according to

$$
\phi_{r}=\phi_{r 2}-\phi_{r 1}=\phi_{I M 2}-\phi_{I M 1}, \quad \phi_{b}=\phi_{b 2}-\phi_{b 1}=-\phi_{I M 2}+\phi_{I M 1} .
$$

This allows for an experimental control of the relative phase by adjusting the phase of the modulation applied to one of the intensity modulators

$$
\delta \phi=2\left(\phi_{I M 2}-\phi_{I M 1}\right)
$$

\subsubsection{Controlling the relative phase of the modulation}

Static potential: One possibility to verify the experimental control over the relative phase $\delta \phi$, is to study the static potential created by the two pairs of beams (red and blue 
a

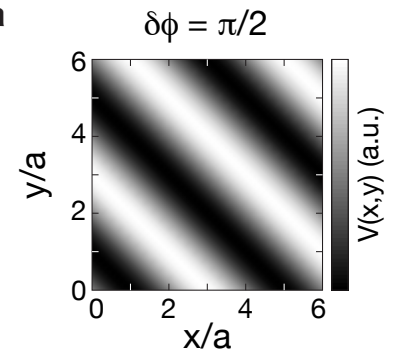

b

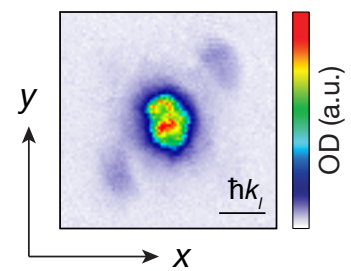

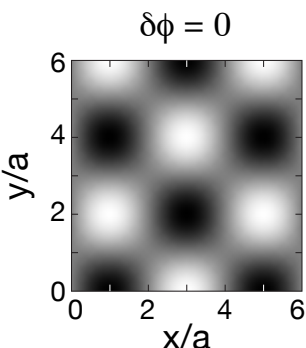

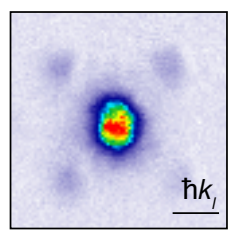

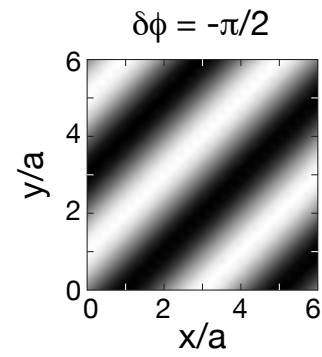

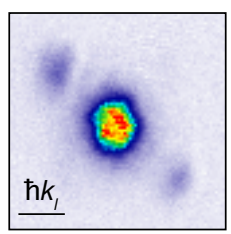

Figure 7.9: Static potential generated by the four beams illustrated as blue and red arrows in Fig. 7.3a for $\omega_{r}=\omega_{b}=0$. a The shape of the potential $V(x, y)$ depends on the relative phase $\delta \phi$ between the two pairs of beams. For $\delta \phi= \pm \pi / 2$ one expects a one-dimensional lattice structure diagonal to the underlying lattice potential with lattice constant $(2 a, \pm 2 a)$. $\mathbf{b}$ Momentum distribution obtained by loading the atoms into the potentials $V(x, y)$ depicted in (a) measured after $10 \mathrm{~ms}$ TOF. For $\delta \phi= \pm \pi / 2$ momentum components at $\left(\hbar k_{l}, \pm \hbar k_{l}\right)$ appear in agreement with a $1 \mathrm{D}$ diagonal lattice.

arrows in Fig. 7.3a), if the frequency differences are set to zero, $\omega_{r}=\omega_{b}=0$. For two particular values of the phase $\delta \phi= \pm \pi / 2$ the resulting static potential corresponds to a one-dimensional lattice, which is diagonal to the normal square lattice (Fig. 7.9a)

$$
V(x, y)= \begin{cases}V_{0} \cos \left(\sqrt{2} k_{l}\left[\hat{\mathbf{e}}_{x}+\hat{\mathbf{e}}_{y}\right]\right) & \text { for } \delta \phi=\pi / 2 \\ V_{0} \cos \left(\sqrt{2} k_{l}\left[\hat{\mathbf{e}}_{x}-\hat{\mathbf{e}}_{y}\right]\right) & \text { for } \delta \phi=-\pi / 2 .\end{cases}
$$

For the experimental results depicted in Fig. $7.9 \mathrm{~b}$ a BEC was loaded within $100 \mathrm{~ms}$ into the static potential and the corresponding momentum distribution was measured after $10 \mathrm{~ms}$ TOF. The relative phase $\delta \phi$ can be reconstructed from these images by comparing them with the calculated lattice potentials depicted in Fig. 7.9a.

Suppression of tunneling in isolated double-wells: To further test the experimental controllability of the setup additional measurements were performed in isolated doublewell potentials similar to the ones described in Sect. 7.1. This time both pairs were applied (Fig. 7.10a) and the two independent modulation potentials were adjusted such that each pair induced resonant tunneling with the same differential modulation amplitude between neighboring sites $\delta V_{r}=\delta V_{b}=V_{0}$. If both of them are applied at the same time, the modulation amplitude between neighboring sites will depend on their relative phase $\delta \phi$ and can be written in the following form 

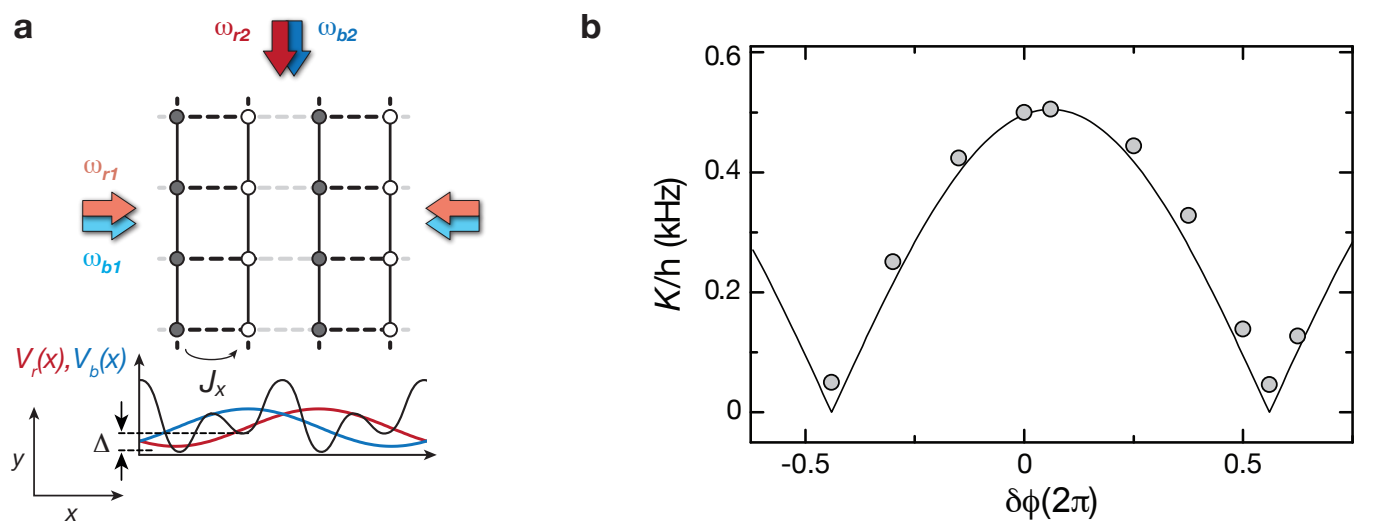

Figure 7.10: Suppression of tunneling in double-well potentials with all four latticemodulation beams. a Schematic drawing of the experimental setup, which consist of a tilted double-well potential along $x$ with energy offset $\Delta \gg J_{x}$ to inhibit tunneling. Resonant tunneling is restored using two pairs of laser beams (red and blue arrows), which create the timedependent potentials $V_{r, b}(x, y, t)$ given in Eq. (7.6). Depending on the relative phase $\delta \phi$, the amplitude of the effective tunnel coupling $K$ varies between zero and $K=J_{x} \mathcal{J}_{1}\left(2 V_{0} / \Delta\right)$. b Measured coupling strength $K$ as a function of the relative phase $\delta \phi$. The solid line is a fit to our data using Eq. (7.26), where the amplitude and phase offset were free fit variables. The absolute value of $\delta \phi$ was not calibrated, which explains the non-zero phase offset.

$$
V_{0}\left[\cos \left(\omega_{r} t\right)+\cos \left(\omega_{b} t+\delta \phi\right)\right]=2 V_{0} \cos \left(\frac{\delta \phi}{2}\right) \cos \left(\omega t+\frac{\delta \phi}{2}\right)
$$

where we have chosen $\omega=\omega_{r}=\omega_{b}=\Delta / \hbar$, which is achieved using the setup illustrated in Fig. 7.11. In the limit $V_{0} / \Delta \ll 1$ the amplitude of the induced tunnel coupling is determined by (Sect. 3.2)

$$
K \simeq J_{x} \frac{V_{0}}{\Delta}\left|\cos \left(\frac{\delta \phi}{2}\right)\right|
$$

The coupling was measured using the same sequence as described in Sect. 7.1 with the final lattice parameters: $V_{x}=7.0(2) \mathrm{E}_{\mathrm{rs}}, V_{x l}=35(1) \mathrm{E}_{\mathrm{rl}}, \varphi_{S L}^{x}=0.073(2) \pi, V_{y}=30(1) \mathrm{E}_{\mathrm{rs}}$ and $V_{z}=30(1) \mathrm{E}_{\mathrm{rz}}$. The energy offset between neighboring sites $\Delta / \hbar \approx 4.43(2) \mathrm{kHz}$ was calibrated independently by performing spectroscopy measurements. Note that for these measurements there is no additional standing wave that might influence the resonance condition because the two terms $V_{\mathrm{sw}}^{r}$ and $V_{\mathrm{sw}}^{b}$ cancel each other for $\varphi_{b}-\varphi_{r}=$ $\pm \pi / 2$. The experimental results are displayed in Fig. $7.10 \mathrm{~b}$ and we obtain an upper limit for the suppression of tunneling $K_{\min } / K_{\max }<0.1$. A residual induced coupling is most likely due to an imperfect back-reflection of the beam along $x$. 

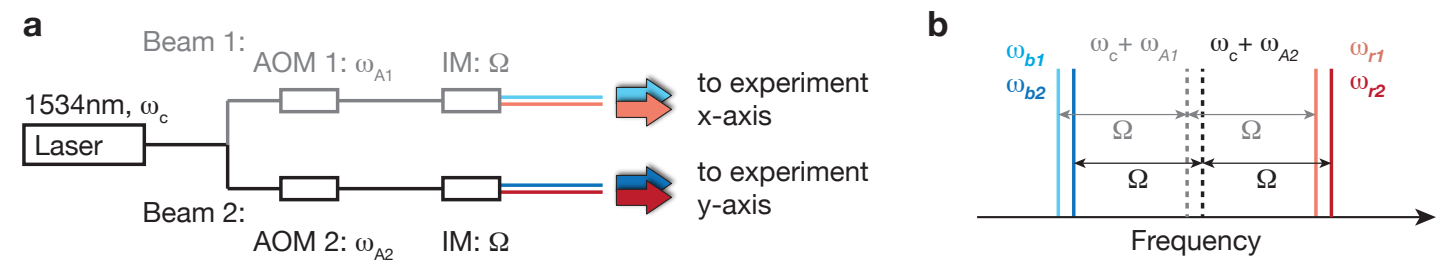

Figure 7.11: Experimental setup for realizing a staggered flux distribution with the beam configuration shown in Fig. 7.3a. a Schematic drawing of the laser setup. The beam is split into two parts (Beam 1 and 2). Each of them passes through an AOM with frequency $\omega_{A 1} / \omega_{A 2}$, and a fiber-coupled IM with frequency $\Omega$. At the output of the IM each beam consists of two frequency components (red and blue). Beam 1 is aligned along the $x$-axis to create the two standing waves and Beam 2 along the $y$-direction to realize the running waves. b Schematic drawing of the frequencies generated in the laser setup shown in (a). Note that the distances are not to scale, $\Omega \gg\left|\omega_{i 2}-\omega_{i 1}\right|$.

\subsection{Staggered flux distribution}

The all-optical laser-assited tunneling scheme introduced in this chapter can be further used to realize a staggered flux distribution by performing a slight modification to the experimental setup (Sect. 7.3). The frequencies for the intensity modulation are set equal $\Omega_{1}=\Omega_{2}=\Omega$ and instead the frequency difference $\Delta / \hbar$ is introduced in the AOM-frequencies $\omega_{A 2}-\omega_{A 1}= \pm \Delta / \hbar$. As a result we obtain $\omega_{r}=\omega_{b}= \pm \Delta / \hbar$ and all bonds are modulated with the same frequency (Fig. 7.11). Together with the fact that the energy offset between neighboring sites alternates in sign the corresponding flux distribution is also alternating in sign $\Phi= \pm(-1)^{m} \pi / 2$. This configuration is very similar to the one discussed in chapter 5 .

Another difference compared to the setup for the uniform flux configuration is the effective coupling $J$ along the perpendicular direction (y-axis). In contrast to Eq. (7.13) it can be written in the following form

$$
\eta_{m, n}^{y}(t)=-\frac{2 V_{0}}{\hbar \omega} \cos \left(-(m+n) \frac{\pi}{2}+\omega t\right) \begin{cases}\sin (\delta \phi / 2), & \text { for } m \text { odd } \\ \cos (\delta \phi / 2), & \text { for } m \text { even }\end{cases}
$$

The extreme values are the same $J_{\max }=J_{y}$ and $J_{\min }=J_{y} \mathcal{J}_{1}\left(2 V_{0} / \Delta\right)$ as shown in Fig. 7.4b. The important difference however is that in this case the inhomogeneity depends on the relative phase $\delta \phi$, which can be controlled experimentally as discussed in the previous section. By setting $\delta \phi=\pi / 2$ the effective coupling is homogeneous independent of the modulation amplitude $V_{0}$. The corresponding value of the renormalized coupling strength would be $J=J_{y} \mathcal{J}_{1}\left(\sqrt{2} V_{0} / \Delta\right)$. 


\section{Chapter 8}

\section{Chern-number measurement of Hofstadter bands}

Bloch bands with non-trivial topology have become available in cold-atom setups with the realization of the Harper-Hofstadter Hamiltonian [70, 71] and the Haldane model [54]. The local structure of the artificial gauge field has been observed previously with local cyclotron orbits $[68,70]$, however, a direct detection of 2D topological invariants remained out of reach.

A first direct detection of a 1D topological invariant has been reported in Ref. [189] using interferometric techniques. This method allows for an extension to $2 \mathrm{D}$ as demonstrated theoretically and experimentally [190, 191]. Several other proposals were developed to directly probe the topology of the system [59] from density plateaus [192], time-of-flight images [193, 194], through semiclassical dynamics [195, 196], hybrid time-of-flight measurements [197] or direct imaging of topological edge states [198].

In this chapter the first measurement of a 2D topological invariant - the Chern number - in a non-electronic system is presented. These observations are based on transport experiments [195, 199] using bosonic atoms. The atomic cloud experiences a transverse motion in response to an externally applied force which is proportional to the Chern number of the band that was populated homogeneously (Sect. 8.2 and Ref. [199]). Through direct imaging of the in-situ center-of-mass position of the cloud in combination with band-population measurements we obtain an experimental value for the Chern number of the lowest band $v_{\exp }=0.99(5)$ (Sect. 8.4). These measurements were facilitated by a newly developed all-optical setup, which was introduced in chapter 7 and realizes the Hofstadter model for a flux $\Phi=\pi / 2$ (Sect. 8.1). The increased experimental flexibility provided by this setup enabled an adiabatic loading of the atoms into the lowest Hofstadter band (Sect. 8.3). 


\subsection{Hofstadter model for a flux $\Phi=\pi / 2$}

The experimental setup for the implementation of the Hofstadter model is illustrated in Fig 8.1a and was described in detail in the previous chapter. Using this scheme laserassisted tunneling on adjacent bonds in the lattice can be controlled individually, which in turn enables flux rectification in a staggered superlattice potential. The method only relies on optical potentials, which are created by far-detuned laser beams.

The basic operation principle can be summarized as follows: Along $x$ bare tunneling is inhibited by a staggered superlattice potential, which introduces an energy offset on every other site. Resonant tunneling is then restored using two pairs of fardetunend beams (red and blue arrows in Fig. 8.1a), with frequency difference $\left|\omega_{i}\right|=$ $\Delta / \hbar, i=\{r, b\}$. Both pairs create a time-dependent potential of the following form $V_{i}(x, y, t)=V_{0} \cos \left(k_{l} x \pm \pi / 4\right) \cos \left(-k_{l} y+\omega_{i} t\right)$, where $k_{l}=\pi /(2 a)$ and $V_{0}$ is the modulation amplitude. This special configuration allows for an independent control of the tunnel coupling on the two kinds of bonds with energy offset $\pm \Delta$ between neighboring sites (red and blue bonds in Fig. 8.1a). For $\omega:=\omega_{r}=-\omega_{b}$ a uniform flux $\Phi= \pm \pi / 2$ per plaquette is realized.

a

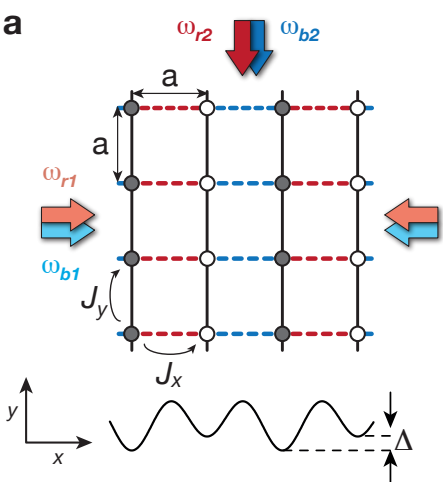

b

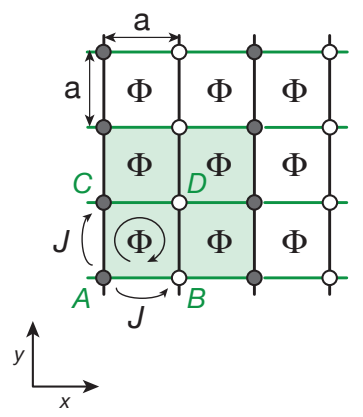

c

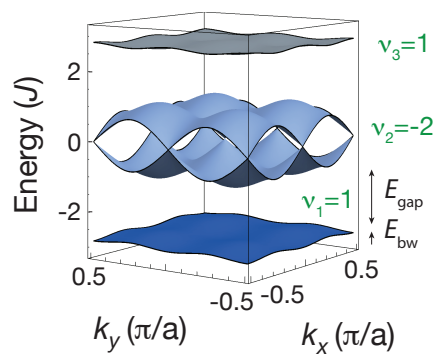

Figure 8.1: Hofstadter model for a flux $\Phi= \pm \pi / 2$ per plaquette. a Schematic drawing of the experimental setup. It consists of a 2D lattice with lattice constant $a=\lambda_{s} / 2$, bare tunnel couplings $J_{x}, J_{y}$ and a staggered potential with energy offset $\Delta \gg J_{x}$ to inhibit tunneling along $x$. The two pairs of beams (red and blue arrows) are used to restore resonant tunneling $\omega_{i}=\omega_{i 2}-$ $\omega_{i 1}= \pm \Delta / \hbar$. b Schematics of the effective time-independent Hamiltonian describing the system shown in (a). The experimental parameters were adjusted such that the effective tunneling along both directions occurs with the same strength $J$. Along $x$ tunneling is additionally accompanied by complex phase factors that lead to a uniform flux $\Phi= \pm \pi / 2$ per plaquette (aligned along the - $\hat{\mathbf{e}}_{z}$ direction). The green shaded area illustrates the magnetic unit cell $A_{M U}=2 a \times 2 a$, where the four sites are denoted as $A, B, C, D$. c Energy spectrum in the FBZ for a lattice with flux $\Phi=\pi / 2$. The lowest tight-binding band splits into four subbands; $E_{\text {gap }} \simeq 1.5 \mathrm{~J}$ denotes the bandgap to the second band and $E_{\mathrm{bw}} \simeq 0.2 \mathrm{~J}$ the bandwidth. The Chern numbers of the three well-separated bands are $v_{\mu}=\{1,-2,1\}$ and were calculated using the methods described in Sect. 2.4. (Figure adapted from Ref. [73]) 
In the high-frequency limit $\hbar \omega \gg J_{x, y}$ the system can be described by the following tight-binding Hamiltonian

$$
\hat{H}=-J \sum_{m, n}\left(\mathrm{e}^{i n \Phi} \hat{a}_{m+1, n}^{\dagger} \hat{a}_{m, n}+\hat{a}_{m, n+1}^{\dagger} \hat{a}_{m, n}+\text { h.c. }\right), \quad \Phi=\pi / 2,
$$

where the Peierls phases are introduced in the Landau gauge and the position in the lattice is defined as $\mathbf{R}=m a \hat{\mathbf{e}}_{x}+n a \hat{\mathbf{e}}_{y}$. A detailed derivation of the effective Hamiltonian in the experimental gauge can be found in Sect. 7.2. The experimental parameters were adjusted such that the effective coupling strengths along $x$ and $y$ are equal. Note, however, that for the experimental parameters $V_{0} /(\hbar \omega)=0.58(2)$ used for the following measurements, the effective coupling along $y$ can be inhomogeneous with variations up to $30 \%$ depending on the initial phase of the modulation $\phi_{0}$ (Sect. 7.2).

Due to the presence of the gauge field the system is no longer invariant under the translation by multiples of the lattice unit vectors. In order to find the new symmetries of the Hamiltonian the magnetic translation symmetries have to be considered (Sect. 2.2). It can be shown that the smallest possible magnetic unit cell contains a magnetic flux of $2 \pi$. For the Hofstadter model with $\Phi=\pi / 2$ this corresponds to a unit cell which is four times larger than the lattice unit cell. Here we have chosen to describe the system using the symmetric magnetic unit cell $A_{M U}=2 a \times 2 a$ (Fig. 8.1b). This choice is not unique and an equivalent description using different unit cells was presented in Sect. 2.2.2. The discrete Schrödinger equation associated with Hamiltonian (8.1) is given by

$$
E \Psi_{m, n}=-J\left(e^{i n \pi / 2} \Psi_{m+1, n}+e^{-i n \pi / 2} \Psi_{m-1, n}\right)-J\left(\Psi_{m, n+1}+\Psi_{m, n-1}\right) .
$$

As discussed in Sect. 2.2 one can make the following ansatz for the wave function

$$
\Psi_{m, n}=e^{i k_{x} m} e^{i k_{y} n} \begin{cases}\psi_{A} e^{i n \pi / 2} & \text { for } m, n \text { odd } \\ \psi_{B} & \text { for } m \text { even, } n \text { odd } \\ \psi_{C} e^{i n \pi / 2} & \text { for } m \text { odd, } n \text { even } \\ \psi_{D} & \text { for } m, n \text { even }\end{cases}
$$

where $k_{x}, k_{y}$ are defined within the FBZ $\left(k_{x} \in\left[-\pi /(2 a), \pi /(2 a)\left[, k_{y} \in[-\pi /(2 a), \pi /(2 a)[)\right.\right.\right.$ and the four sites of the unit cell are denoted as $A, B, C, D$ (Fig. 8.1b). Inserting this ansatz into the Schrödinger equation above reduces the problem to a four-dimensional eigenvalue equation

$$
\hat{H}\left(\begin{array}{c}
\psi_{A} \\
\psi_{B} \\
\psi_{C} \\
\psi_{D}
\end{array}\right)=E(\mathbf{k})\left(\begin{array}{c}
\psi_{A} \\
\psi_{B} \\
\psi_{C} \\
\psi_{D}
\end{array}\right)
$$

with 


$$
\hat{H}=-2 J\left(\begin{array}{cccc}
0 & i \sin \left(k_{x} a\right) & -\sin \left(k_{y} a\right) & 0 \\
-i \sin \left(k_{x} a\right) & 0 & 0 & \cos \left(k_{y} a\right) \\
-\sin \left(k_{y} a\right) & 0 & 0 & \cos \left(k_{x} a\right) \\
0 & \cos \left(k_{y} a\right) & \cos \left(k_{x} a\right) & 0
\end{array}\right) .
$$

The corresponding energy spectrum consists of four subbands, where the two middle bands touch at four Dirac cones (Fig. 8.1c). Hence, the spectrum is decomposed into three well separated Hofstadter bands. The lowest band exhibits a Chern number $v_{1}=$ +1 and is topologically equivalent to the lowest Landau level. Moreover it exhibits a large flatness ratio of $E_{\mathrm{gap}} / E_{\mathrm{bw}} \simeq 7$, which makes this system a promising candidate to realize fractional Chern insulators [27].

\subsection{Anomalous Hall velocity and Chern-number measurement}

In Sect. 2.4 the connection between the quantized Hall conductance and the topological invariants characterizing the energy gaps were established for electronic systems. If the Fermi energy lies in a spectral gap, the bulk is insulating and the quantized values of the Hall conductivity are directly related to the number of metallic edge states. In a sense these measurements probe the topological properties of the edge states, which are related to the bulk via the bulk-edge correspondence [10, 11].

Cold atoms in optical lattices may offer new possibilities to directly probe the topological properties of the bulk energy bands. It has been shown that transport measurements could allow for the observation of the anomalous Hall velocity, which is proportional to the Berry curvature [94], and might be used to determine the Chern number of the corresponding energy band [59, 195, 199]. In the presence of a constant external force $\mathbf{F}=F \hat{\mathbf{e}}_{y}$ the atoms undergo Bloch oscillations in the direction of the force [200], which is captured by the band velocity $\mathbf{v}_{\mu}^{\text {band }}=\partial_{\mathbf{k}} E_{\mu} / \hbar, \mu$ being the band index. If additionally the band exhibits a non-zero Berry curvature $\Omega_{\mu}(\mathbf{k})$ as defined in Eq. (2.46), the atoms will experience a perpendicular Hall drift $v_{\mu}^{x}$ (Fig. 8.2a). For a particle in a state $\left|u_{\mu}(\mathbf{k})\right\rangle$ of the $\mu$ th band the velocity is given by [94]

$$
\begin{aligned}
v_{\mu}^{y}(\mathbf{k}) & =\frac{1}{\hbar} \partial_{k_{y}} E_{\mu} \\
v_{\mu}^{x}(\mathbf{k}) & =\frac{1}{\hbar} \partial_{k_{x}} E_{\mu} \underbrace{-\frac{F}{\hbar} \Omega_{\mu}(\mathbf{k})}_{\text {anomalous velocity }} .
\end{aligned}
$$

Recently, the anomalous Hall velocity was observed experimentally with ultracold atoms in a modulated honeycomb lattice [54] and in an electronic system using graphene superlattices [201].

For the following measurements we make use of this anomalous velocity to determine 

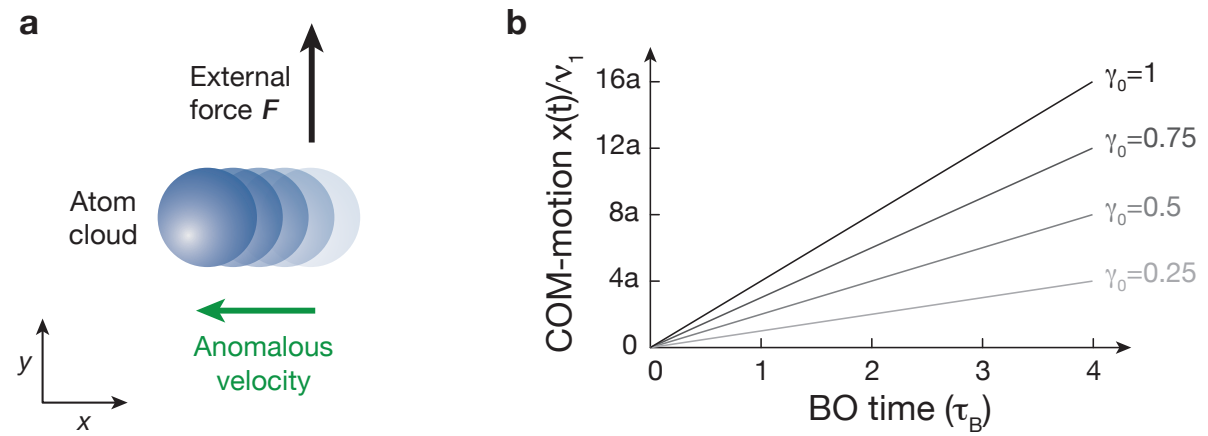

Figure 8.2: Schematic drawing of the Chern-number measurement. a In the presence of an external force the atoms experience a net perpendicular Hall drift, determined by the anomalous velocity $v_{\mu}^{x}(\mathbf{k})$ in Eq. (8.6). For a filled band the mean displacement is proportional to the Chern number $v_{\mu}$ of the occupied band $\mu$, which can be determined by measuring the transverse centerof-mass motion of the atomic cloud. (Figure adapted from Ref. [73]) $\mathbf{b}$ Transverse motion as a function of the Bloch oscillation (BO) time. For the Hofstadter model for $\Phi=\pi / 2$ the transverse deflection $x(t)$ in Eq. (8.14) is only determined by the Chern number of the lowest band $v_{1}$ and the band filling factor $\gamma_{0}$. If only the lowest band is filled it takes a maximum value $\gamma_{0}=1$ and it decreases to zero with increasing higher-band population. For a homogeneous filling of all the bands $\gamma_{0}=0$ the cloud is not deflected in the transverse direction, consistent with an effective Chern number of zero.

the Chern number of the lowest Hofstadter band experimentally with bosonic atoms. In order to isolate the anomalous velocity from the trivial effects associated with the band velocity $\mathbf{v}_{\mu}^{\text {band }}=\partial_{\mathbf{k}} E_{\mu} / \hbar$ we consider a uniformly filled band, where $\rho_{\mu}(\mathbf{k}) \equiv \rho_{\mu}$ is the particle density per momentum $\mathbf{k}$ and thus per state $\left|u_{\mu}(\mathbf{k})\right\rangle$. Due to the symmetry of the dispersion relation the contributions from the band velocity identically vanish. For an infinite system the mean band velocity per particle of a uniformly filled $\mu$ th band can be computed according to

$$
\left\langle v_{\mu}^{\text {band }}\right\rangle_{x}=\frac{\rho_{\mu}}{\hbar} \int_{\mathrm{FBZ}} \partial_{k_{x}} E_{\mu} \mathrm{d}^{2} k=0, \quad\left\langle v_{\mu}^{\text {band }}\right\rangle_{y}=\frac{\rho_{\mu}}{\hbar} \int_{\mathrm{FBZ}} \partial_{k_{y}} E_{\mu} \mathrm{d}^{2} k=0,
$$

where the integration is performed over all momenta in the first magnetic Brillouin zone (FBZ). The expressions vanish because for every velocity $\mathbf{v}$ there exists a counterpart with opposite velocity $-\mathbf{v}$. For Fermions filled bands can be obtained by setting the Fermi energy within a spectral gap [199]. Here we consider an incoherent distribution of bosonic atoms which populate the band homogeneously in $\mathbf{k}$-space. This was verified independently in the experiment as will be shown in the following section. Similarly, the mean anomalous velocity of the $\mu$ th band is determined by 


$$
\left\langle v_{\mu}^{x}(\mathbf{k})\right\rangle=-\frac{\rho_{\mu} F}{\hbar} \underbrace{\int_{\mathrm{FBZ}} \Omega_{\mu}(\mathbf{k}) \mathrm{d}^{2} k}_{\rightarrow 2 \pi v_{\mu}},
$$

where $v_{\mu}$ is the Chern number of the $\mu$ th band. For well separated non-degenerate energy bands the particle density in each band $\mu$ is given by

$$
\rho_{\mu}=\frac{N_{\mu}}{N} \frac{1}{A_{\mathrm{FBZ}}}=\eta_{\mu} \frac{a^{2}}{\pi^{2}}, \quad \eta_{\mu}:=\frac{N_{\mu}}{N}
$$

where $N_{\mu}$ is the particle number in each band, $N=\sum_{\mu} N_{\mu}$ is the total atom number and $A_{\mathrm{FBZ}}=\pi^{2} / a^{2}$ is the area of the first magnetic Brillouin zone. Assuming that the band populations remain constant during the dynamics $\eta_{\mu} \equiv \eta_{\mu}^{0}$ the contribution of the $\mu$ th band to the center-of-mass displacement (COM) of the atomic cloud perpendicular to the force follows the relation

$$
x_{\mu}(t)=\eta_{\mu}^{0} \frac{4 a^{2} F}{h} v_{\mu} t=-4 a \eta_{\mu}^{0} v_{\mu} \frac{t}{\tau_{B}},
$$

where $\tau_{B}=h /(F a)$ is the characteristic timescale for Bloch oscillations. If only the lowest band is filled $\eta_{1}=1$ and $\eta_{\mu>1}=0$ we expect a deflection of $4 a v_{1}$ per Bloch oscillation period (Fig. 8.2b)

$$
x_{1}(t)=-4 a v_{1} \frac{t}{\tau_{B}}
$$

and the Chern number of the lowest band $v_{1}$ can be simply extracted from the slope of the linear displacement perpendicular to the force. The displacement after one Bloch oscillation period is expected to be on the order of four lattice constants, which is large enough to be detectable with conventional imaging systems. In our experiment the magnification of the imaging system is chosen such that one pixel corresponds to about four lattice constants. The imaging resolution is not very important since we are only interested in the COM motion.

For the particular case of the Hofstadter model for $\Phi=\pi / 2$ the spectrum is split into three well separated bands where the middle "super"-band consists of two subbands (Fig. 8.1c). This middle super-band contains twice as many states because there are two available states for each momentum $\mathbf{k}$ and the particle density in this band is given by $\rho_{2}=\eta_{2} /\left(2 A_{\mathrm{FBZ}}\right)$. Taking into account the contribution of all bands the resulting COM-motion can be written as

$$
x(t)=\sum_{\mu} x_{\mu}(t)=-4 a \frac{t}{\tau_{B}}\left(\eta_{1}^{0} v_{1}+\frac{\eta_{2}^{0}}{2} v_{2}+\eta_{3}^{0} v_{3}\right) .
$$

Using the fact that the Chern number associated with the total tight-binding band necessarily vanishes, $\sum_{\mu} v_{\mu}=0$, the equation above simplifies 


$$
x(t)=-4 a \frac{t}{\tau_{B}}\left[\left(\eta_{1}^{0}-\eta_{3}^{0}\right) v_{1}+\left(\eta_{2}^{0}-2 \eta_{3}^{0}\right) \frac{v_{2}}{2}\right] .
$$

We can further make use of the particle-hole symmetry inherent to the Hofstadter model as discussed in Section 2.3.2, which tells us, that the Chern number distribution is symmetric around $E=0$ such that the Chern number of the lowest band is equal to the one of the highest band $v_{1}=v_{3}=-v_{1}-v_{2}$ (Sect. 2.4). This allows us to write the COM-motion as a function of the Chern number of the lowest band only

$$
x(t)=-4 a \frac{t}{\tau_{B}} \gamma_{0} v_{1}, \quad \gamma_{0}=\eta_{1}^{0}-\eta_{2}^{0}+\eta_{3}^{0},
$$

where we have introduced the filling factor $\gamma_{0}$. The maximum value of the filling factor equals one, if all atoms populate the lowest or highest Hofstadter band respectively and equals zero if all the bands are occupied homogeneously. In this case the atomic cloud would not experience a transverse COM displacement (Fig. 8.2b).

\subsection{Adiabatic loading into the Hofstadter bands}

Loading the atoms into the lowest Hofstadter band is a highly non-trivial task by itself. Let us consider that the experimental sequence starts by loading the atoms in a normal, topologically trivial square lattice with lattice constant $a$. By simply turning on the flux lattice, the lowest band splits into four subbands and the atoms might be transferred to higher Hofstadter bands. To overcome this issue a loading sequence was developed based on additional staggered potentials along the two directions in the horizontal plane, which connects the band population of a topologically trivial band to the one of a topologically non-trivial band in a controlled way. Here we benefit from the increased experimental control facilitated by the all-optical setup (chapter 7) compared to the one where a magnetic field gradient was used (chapter 6).

\subsubsection{Loading sequence: general idea}

The underlying idea is to first load the atoms into a lattice which has a unit cell that is equivalent to the magnetic unit cell depicted in Fig. 8.1b. This can be done by introducing an additional term $\hat{H}_{d}$ in the Hamiltonian, which corresponds to a staggered detuning $\delta$ along both directions (Fig. 8.3a)

$$
\hat{H}_{d}=\sum_{m, n}\left[(-1)^{m} \frac{\delta_{x}}{2}+(-1)^{n} \frac{\delta_{y}}{2}\right] \hat{n}_{m, n},
$$

with $\delta:=\delta_{x}=\delta_{y}$. Along $x$ the additional detuning increases the potential away from the resonance condition to $\Delta+\delta$, while along $y$ it is simply given by $\delta$. In the limit $\Delta \gg \delta$ this results in the following modified Hamiltonian 
a

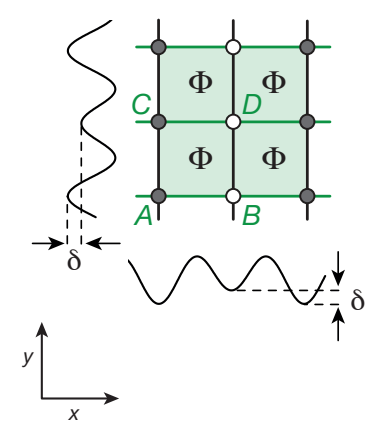

b

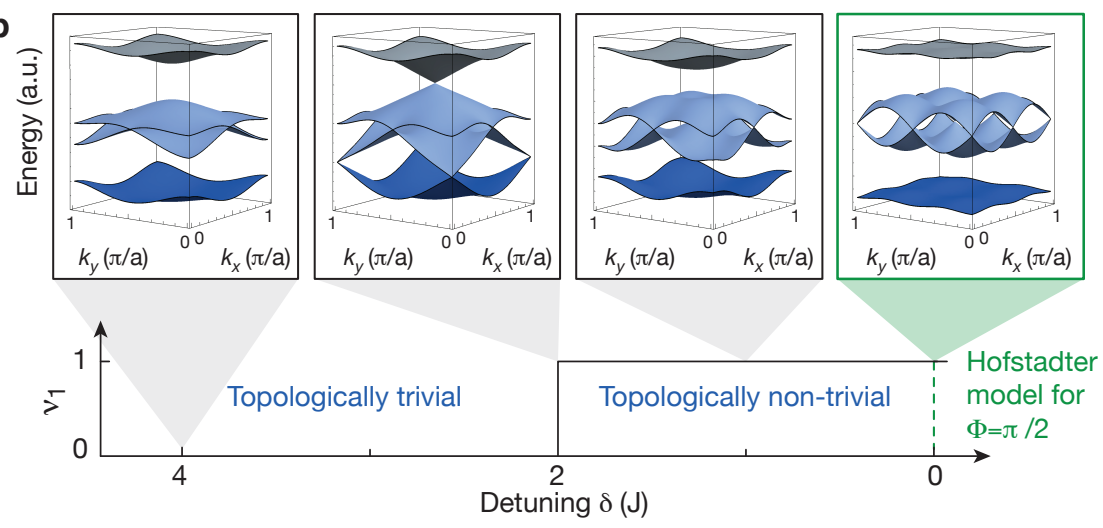

Figure 8.3: Energy spectra and topology of the Bloch bands as a function of the staggered detuning $\delta$. a Illustration of the Hofstadter-like optical lattice with additional staggered detuning $\delta$ along the $x$ - and $y$-direction. The unit cell of the lattice is depicted by the green shaded area and the four non-equivalent sites are labeled as $A, B, C, D$. b Energy spectra as a function of the detuning. For a detuning larger than $2 J$ the bands are topologically trivial and the Chern numbers are zero. At $\delta=2 J$ there is a topological phase transition, where the gaps in the spectrum close and the system enters the topologically non-trivial regime. In this regime the Chern number of the lowest band is $v_{1}=+1$ for $\Phi=\pi / 2$. For vanishing detuning $\delta=0$ the system realizes the Hofstadter model with flux $\Phi=\pi / 2$. Note that the energy axis is rescaled for each spectrum. (Figure adapted from Ref. [73])

$$
\hat{H} \rightarrow \hat{H}=-2 J\left(\begin{array}{cccc}
\delta /(2 J) & i \sin \left(k_{x} a\right) & -\sin \left(k_{y} a\right) & 0 \\
-i \sin \left(k_{x} a\right) & 0 & 0 & \cos \left(k_{y} a\right) \\
-\sin \left(k_{y} a\right) & 0 & 0 & \cos \left(k_{x} a\right) \\
0 & \cos \left(k_{y} a\right) & \cos \left(k_{x} a\right) & -\delta /(2 J)
\end{array}\right)
$$

During the loading sequence the detuning is decreased to zero, $\delta \rightarrow 0$. The corresponding energy spectra are displayed in Fig. 8.3b. Since the unit cells are equivalent, the number of bands is preserved during the loading sequence. For $\delta>2 J$ the topology of the bands is trivial and all Chern numbers are zero. At $\delta=2 J$ a topological phase transition occurs and the gaps in the spectrum close. For $\delta<2 J$ the topologically non-trivial regime is reached, where the lowest band has a Chern number of $v_{1}=+1$, and at the end of the sequence $(\delta=0)$ the Harper-Hofstadter Hamiltonian for $\Phi=\pi / 2$ is realized. Note that the horizontal axes of the energy spectra in Fig. 8.3 are different compared to the ones shown in Ref. [73]. Depending on the definition of the gauge and the detuning term given in Eq. (8.15) the dispersion relation might be shifted in momentum space but this has no impact on the general loading scheme. 


\subsubsection{Experimental sequence}

The experimental sequence is illustrated in Fig. 8.4. It started by loading a Bose-Einstein condensate of ${ }^{87} \mathrm{Rb}$ atoms within $150 \mathrm{~ms}$ into a two-dimensional optical superlattice of depths $V_{x}=6.0(2) \mathrm{E}_{\mathrm{rs}}, V_{x l}=5.25(16) \mathrm{E}_{\mathrm{rl}}, V_{y}=10(1) \mathrm{E}_{\mathrm{rs}}$ and $V_{y l}=1.75(5) \mathrm{E}_{\mathrm{rl}}$. The phases between the short- and long-lattice standing waves, $\varphi_{S L}^{x}=\varphi_{S L}^{y}=\pi / 2$ (Sect. 4.3), where chosen so as to create a staggered potential with energy offset $\Delta+\delta_{x}$ along $x$ and $\delta_{y}$ along $y$. Along the $z$-direction the atoms were confined by a weak harmonic potential generated by a crossed optical dipole trap in the horizontal plane, $\omega_{z} /(2 \pi) \approx 20 \mathrm{~Hz}$. Initially tunneling was inhibited along both directions due to the potential detuning $\left(\Delta+\delta_{x}\right) \gg J_{x}$ and $\delta_{y} \gg J_{y}$ and all atoms occupied the low-energy sites, denoted as $A$-sites (Fig. 8.3a). Then, the modulation was switched on off-resonant within $30 \mathrm{~ms}$ with $V_{0} \simeq 1.6 \mathrm{E}_{\mathrm{rl}}$ and $\omega /(2 \pi)= \pm \Delta / h \simeq \pm 2.7 \mathrm{kHz}$. The resonance condition was calibrated independently for the final lattice parameters along $x, V_{x}=6.0(2) \mathrm{E}_{\mathrm{rs}}$ and $V_{x l}=3.25(10) \mathrm{E}_{\mathrm{rl}}$, by performing spectroscopy measurements as discussed in Sect. 4.3.2. The detuning was chosen larger than the effective coupling strength on resonance $\delta_{x} \gg J$ such that tunneling remained suppressed along this direction. Additionally along the $y$ direction the condition $\delta_{y} \ll \Delta$ was fulfilled in order to assure that the modulation with frequency $\hbar \omega=\Delta$ did not induce tunneling in the perpendicular direction (Fig. 8.4b). In these limits tunneling was suppressed in both directions and atoms stayed in $A$-sites. The values for $\delta_{x}$ and $\delta_{y}$ were optimized experimentally such that less than $10 \%$ of the atoms were transferred to higher bands after switching on the modulation.

The loading into the Hofstadter bands was achieved by ramping down the detunings to zero within $30 \mathrm{~ms}$, by changing the long lattice depth along $x$ to $V_{x l}=3.25(10) \mathrm{E}_{\mathrm{rl}}$ and the long lattice along $y$ to $V_{y l}=0 \mathrm{E}_{\mathrm{rl}}$. For these values resonant tunneling occurred along both directions and the parameters were chosen such that the effective coupling strengths along both directions were the same, $J=75(3) \mathrm{Hz}$. This lattice configuration realizes a lattice with uniform flux $\Phi= \pm \pi / 2$ per plaquette, where the direction of the flux depends on the sign of the frequency $\omega$.

\subsubsection{Momentum distribution and initial band population}

The Chern-number measurement as sketched in Sect. 8.2 is based on the assumption that the atoms in each Hofstadter band populate the corresponding band homogeneously in $\mathbf{k}$-space. This assumption was verified experimentally by measuring the momentum distribution in the different bands.

For this purpose the loading sequence described above (Fig. 8.3) was reversed. The sequence started by ramping up the staggered detunings $\delta_{x}$ and $\delta_{y}$ within $30 \mathrm{~ms}$ in order to suppress tunneling along both directions. The final lattice depths were $V_{x}=6.0(2) \mathrm{E}_{\mathrm{rs}}$, $V_{x l}=5.25(16) \mathrm{E}_{\mathrm{rl}}, V_{y}=10(1) \mathrm{E}_{\mathrm{rs}}$ and $V_{y l}=1.75(5) \mathrm{E}_{\mathrm{rl}}$. The number of energy bands is preserved during this ramp and the populations of the topological Hofstadter bands are 

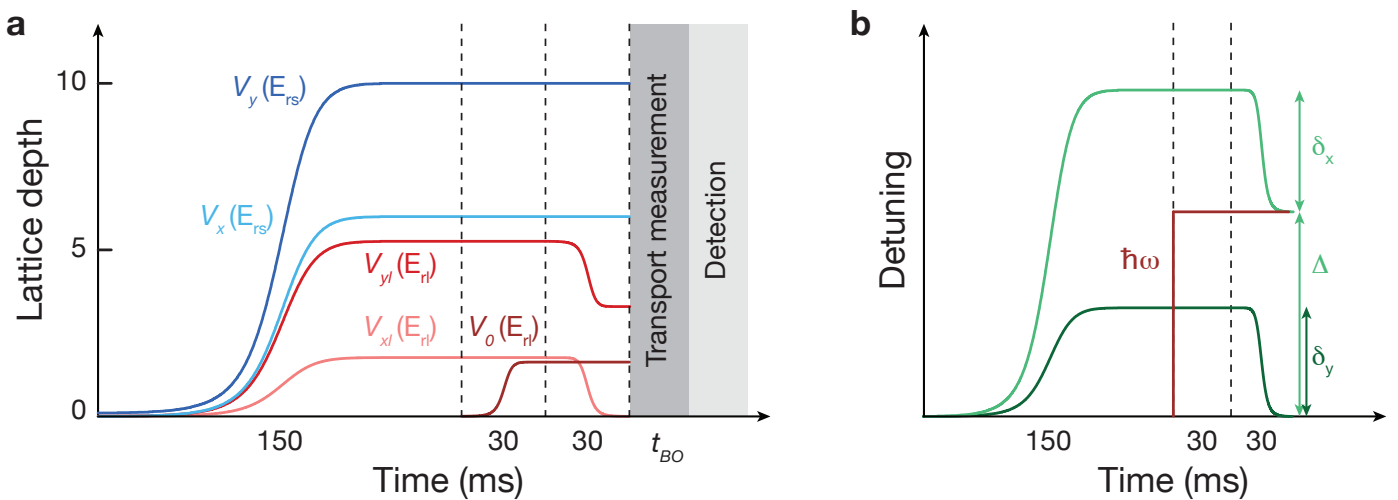

Figure 8.4: Schematic drawing of the experimental sequence. a Lattice depths as a function of time. Note that the distances on the time-axis are not to scale. After loading the atoms into the Hofstadter bands as described in the main text the transport measurements were performed for a certain Bloch oscillation time $t_{B O}$ before several detection techniques were applied: in-situ position of the cloud, band-population measurements, momentum distribution (Sect. 8.3.3). b Staggered energy offsets along $x$ and $y$ as they evolve during the sequence (green). For comparison the modulation frequency $\hbar \omega$ is shown in red.

mapped onto the topologically trivial Hofstadter-like bands.

Due to the detuning tunneling was suppressed and the modulation could be switched off instantaneously to map the populations of the explicitly time-dependent Hamiltonian onto the ones of the static superlattice potential with staggered offsets $\Delta+\delta_{x}$ and $\delta_{y}$. The size of the Brillouin zone is unchanged during the whole mapping sequence, hence, the population of different $k$-states is preserved if scattering processes and heating effects during the ramp are neglected. Consequently the momentum distribution of the Hofstadter bands is reflected in the momentum distribution of the static twodimensional superlattice potential.

All lattice potentials are subsequently ramped down adiabatically to map the momentum distribution in the lattice onto the real-space momentum distribution. Then the atoms were released from the trap and detected via absorption imaging after $10 \mathrm{~ms}$ TOF (Fig. 8.5a). The connection between the different Brillouin zones and the corresponding Hofstadter bands is illustrated in Fig. $8.5 \mathrm{~b}$ and c. There are two informations we obtain from these images: (a) we achieve typically a population of about $60 \%$ in the lowest Hofstadter band; (b) the distribution is homogeneous in each of the individual bands. This data is consistent with the assumption of homogeneous band populations.

In principle the band populations can be inferred by counting the atom numbers in the different Brillouin zones. However, the zones are connected, thus, to ease the counting of the occupations we apply a slightly different sequence that separates the different Brillouin zones from each other (Sect. 8.4.2). 

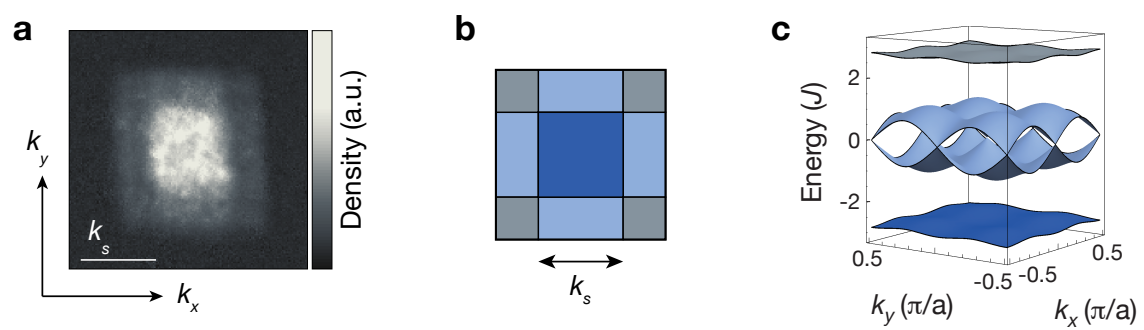

Figure 8.5: Momentum distribution of the atoms in the three well-separated Hofstadter bands. a Measured momentum distribution after loading the atoms into the Hofstadter bands using the sequence described above and applying a subsequent band-mapping sequence as discussed in this section. The distribution was detected after $10 \mathrm{~ms}$ TOF. The data was averaged over 15 independent measurements. b Schematics of the corresponding Brillouin zones. c Energy spectrum of the Hofstadter model for $\Phi=\pi / 2$. The color code illustrates the connection between the Brillouin zones and the associated Hofstadter bands. (Figure adapted from Ref. [73])

\subsection{Transport measurements}

In the following section direct measurements of the anomalous velocity as a response to an optical force are presented. The evolution was tracked by taking in-situ images of the atomic cloud. We observe that for short times the deflection of the atomic cloud is indeed linear in time in agreement with Eq. (8.14) and exact diagonalization studies $[195,199]$. For longer times the transverse motion saturates due to a repopulation of the atoms between different Hofstadter bands, which leads to a time-dependent filling factor $\gamma_{0} \rightarrow \gamma(t)$. In combination with independent band-population measurements we were able to extract an experimental value for the Chern number of the lowest band $v_{\exp }=0.99(5)$ from from this long-time dynamics.

Optical force: The Bloch oscillations along $y$ were induced using an optical gradient. It was realized with an additional laser beam with wavelength $\lambda_{z}=844 \mathrm{~nm}$, which was focused at the atom position to a waist of about $125 \mu \mathrm{m}$. The beam was aligned relative to the position of the atomic cloud along $y$ such that the cloud was located at the maximum slope of the Gaussian beam profile. Along $x$ the beam was centered on the atom position in order not to induce Bloch oscillations along that axis. The strength of the optical gradient was calibrated independently through Bloch oscillations in a onedimensional optical lattice with $V_{y}=10(1) \mathrm{E}_{\mathrm{rs}}$.

Experimental observable: After applying the optical force, the COM evolution of the cloud was measured in-situ for opposite directions of the flux $\Phi= \pm \pi / 2$. These positions were then subtracted to obtain the differential shift 


$$
x(t, \pi / 2)-x(t,-\pi / 2)=2 x(t) .
$$

This quantity is less susceptible to systematic errors and experimental uncertainties of the absolute position of the atomic cloud caused by slow drifts. For each dataset we averaged ten in-situ images of the atomic cloud for each direction of the flux $\Phi$ and evaluated the corresponding COM positions $x(t, \pm \pi / 2)$. The images were taken alternating for the two directions. A typical result after subtracting the averaged images is shown in Fig. 8.6a.

\subsubsection{Short-time dynamics}

For short times, where inter-band transitions are negligible, we observe an almost linear evolution of the differential shift Fig. 8.6b. Taking into account the measured filling factor $\gamma_{0}$ and using the assumption that the band populations $\eta_{\mu}^{0}$ remain constant for short times we can compare the short-time trajectories with the equation-of-motion (8.14) to extract a first experimental value of the Chern number of the lowest band. Fitting a linear function to our data we obtain $v_{\exp }=0.9(2)$. As shown in Fig. 8.6b deviations from the ideal evolution are partially captured by the numerical simulations which also account for Landau-Zener transitions to higher Hofstadter bands during the dynamics.

\section{Numerical simulations}

The ideal evolution governed by Eq. (8.14) is valid for weak forces and short times, where the band populations can be assumed to remain constant $\eta_{\mu}(t)=\eta_{\mu}^{0}$. In order to gain more inside into the dynamics we simulated the full non-interacting problem using the effective Hamiltonian which can be written in the following form

$$
\hat{H}_{\text {eff }}=-J \sum_{m, n}\left\{\hat{a}_{m+1, n}^{\dagger} \hat{a}_{m, n} e^{i\left[\pi / 2(m+n)-\phi_{0}\right]}+\left(1+f_{m, n}\right) \hat{a}_{m, n+1}^{\dagger} \hat{a}_{m, n}+\text { h.c. }\right\}+\hat{V}_{\text {conf }},
$$

where the experimental gauge was introduced as derived in Sect. 7.2 for a relative phase $\delta \phi=-\pi / 2$. The initial phase of the modulation $\phi_{0}$ appears in the Peierls phases as well as in the coupling terms along $y$ as defined in Eq. (7.15). In addition the harmonic

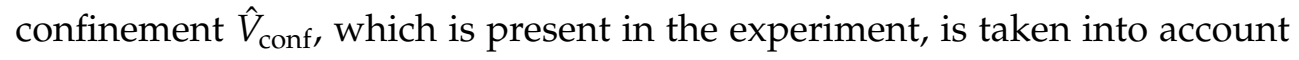

$$
\hat{V}_{\text {conf }}=\sum_{m, n}=V_{m, n} \hat{n}_{m, n}, \quad V_{m, n}=\kappa\left(0.5 m^{2}+n^{2}\right),
$$

where $\kappa=10^{-3} J$. The trap frequencies were calibrated independently and are given by $\omega_{x} \approx 2 \pi \times 7.5 \mathrm{~Hz}$ and $\omega_{y} \approx 2 \pi \times 10.5 \mathrm{~Hz}$.

The numerical simulations were performed similar to the ones described in Ref. [199]. The initial conditions are first established by confining the system within a certain radius $r_{0} \sim 10-30 a$ using a potential $\hat{V}_{\text {initial }} \propto\left(r / r_{0}\right)^{\zeta}$, with $\zeta \gg 10$. Using an abrupt 
a

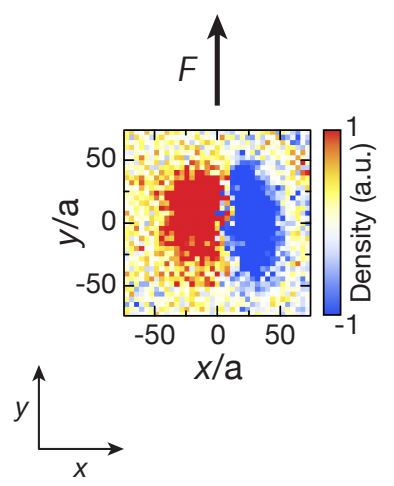

b

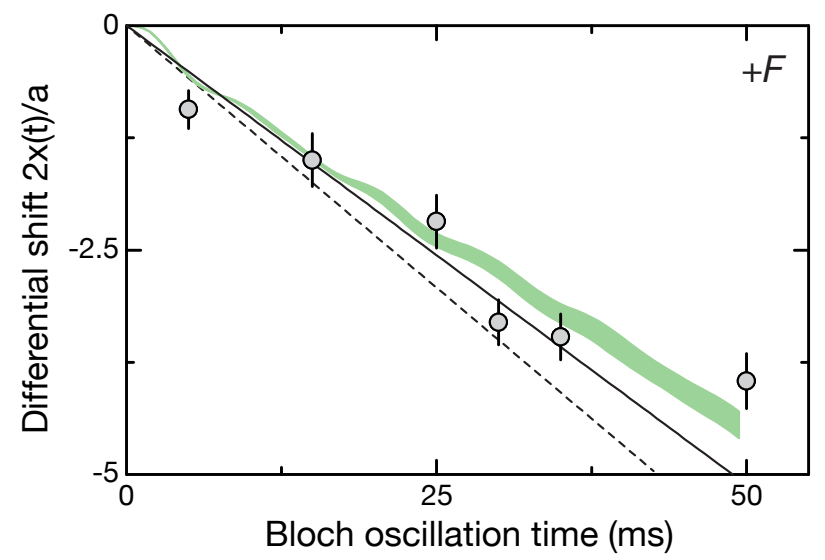

Figure 8.6: Differential COM displacement of the atomic cloud versus Bloch oscillation time. The optical gradient was aligned along $y, \mathbf{F}=+F \hat{\mathbf{e}}_{y}$, with $F a / h=38.4(8) \mathrm{Hz}$. a Typical experimental image obtained after subtracting the averaged in-situ images of the atomic cloud for opposite directions of the flux $\pm \Phi$ of one dataset as defined in the main text. All images were taken at $t=35 \mathrm{~ms}$. $\mathbf{b}$ The gray data points depict the measured differential COM displacement. Each data point is an average of five datasets and the error bars depict the standard error of the mean. The black solid line is a linear fit to the data for $t \leq 35 \mathrm{~ms}$. Taking into account the measured initial band populations $\eta_{\mu}^{0}=\{0.55(6), 0.31(3), 0.13(3)\}$ and using Eq. (8.14) yields $v_{\exp }=0.9(2)$. The dashed line shows the ideal evolution for the measured initial band populations and $v_{1}=1$. The green shaded area depicts numerical simulations taking into account the inhomogeneities in the coupling along $y$ according to Hamiltonian (8.18) for the initial phase of the driving $\phi_{0} \in[0, \pi]$ as introduced in Sect. 7.2. (Figure adapted from Ref. [73])

circular potential simplifies the analysis, however, as shown in Ref. [199] smoother potentials could be also considered for the initial state preparation. First the Hamiltonian $\hat{H}_{\text {eff }}+\hat{V}_{\text {initial }}$ is diagonalized on a finite size system with radius $r>r_{0}$. The band structure of the Harper-Hofstadter Hamiltonian is clearly visible in the density of states and the eigenstates can be classified in terms of these bulk bands. After setting the initial band populations (here $\eta_{1}=1$ and $\eta_{\mu>1}=0$ ) the time evolution of the states is calculated according to $\hat{H}_{\text {eff }}+\hat{V}_{\text {force, }}$ where $\hat{V}_{\text {force }}$ describes the force $\mathbf{F}=+F \hat{\mathbf{e}}_{y}$ acting on the particles along the $y$-direction according to

$$
\hat{V}_{\text {force }}=-F a \sum_{m, n} n \hat{n}_{m, n}
$$

The COM deflection $x(t)$ is then obtained by computing the spatial density for each evolved state.

The numerical results are plotted in Fig. 8.7 for two different values of the force $F_{\exp }=$ $0.52 \mathrm{~J} / a$ (dark green) and $F_{\text {weak }}=0.25 \mathrm{~J} / a$ (light green). For the weaker force the evolution follows the linear behavior predicted by Eq. (8.14) and the band populations $\eta_{\mu}(t)=\eta_{\mu}^{0}$ remain approximately constant during the evolution. For the larger force $F_{\text {exp }}$ 


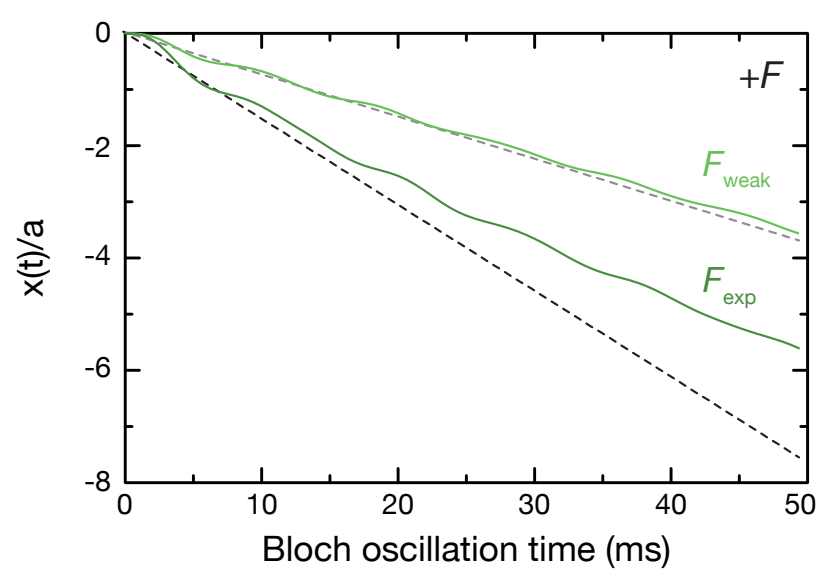

Figure 8.7: Numerical simulations of the transverse COM motion $x(t)$ for $\mathbf{F}=+F \hat{\mathbf{e}}_{y}, \Phi=\pi / 2$ and $\gamma_{0}=1$. The dynamics is governed by the Hamiltonian (8.18). The numerical calculations were performed for $\phi_{0}=\pi / 4$ and the experimental parameter $V_{0} /(\hbar \omega)=0.58$. For this value of the phase the effective coupling along $y$ is uniform given by $J \approx 0.83 J_{y}$ (Sect. 7.2) and the effective coupling along $x$ was set to $K=J_{y}$. The solid green lines show the results for the force $F_{\exp }=0.52 \mathrm{~J} / a$ (dark green) used for the experiments shown in Fig. 8.6 and for a weaker force $F_{\text {weak }}=0.25 \mathrm{~J} / a$ (light green). The dashed lines show the corresponding ideal evolutions according to the equation-of-motion (8.14). (Figure adapted from Ref. [73])

as used for the measurements presented above, the numerical simulations show a clear deviation from the ideal evolution due to Landau-Zener transitions to higher bands. Such effects are neglected in the simple equation-of-motion (8.14) and lead to band repopulation $\eta_{\mu}(t)$ during the dynamics. The simulations shown in Fig. $8.6 \mathrm{~b}$ additionally take into account the measured initial filling factor $\gamma_{0} \approx 0.36$, which leads to a further reduction of the transverse motion because atoms that populate the middle band with Chern number $v_{2}=-2 v_{1}$ experience a drift opposite to those that populate the lowest Hofstadter band. For short times the numerical simulations, which consider both, the initial band population and the Landau-Zener transitions, are in agreement with the experimental data for times $t \leq 35 \mathrm{~ms}$ (Fig. 8.6b). The trajectories for different values of the phase $\phi_{0}$ are found to be similar for our experimental values $V_{0} /(\hbar \omega)=0.58(2)$. In conclusion the reduced value of the experimental Chern number $v_{\exp }=0.9(2)$ can be partially attributed to Landau-Zener transitions as they are not captured by the simple model given in Eq. (8.14).

\subsubsection{Long-time dynamics and band populations}

In the following section we present a more precise determination of the Chern number based on the long-time dynamics combined with independent band-population measurements. We observe that the transverse motion saturates already after about $2 \tau_{b} \approx 50 \mathrm{~ms}$ and is symmetric with respect to the direction of the force $\mathbf{F}= \pm F \hat{\mathbf{e}}_{y}$ (black 


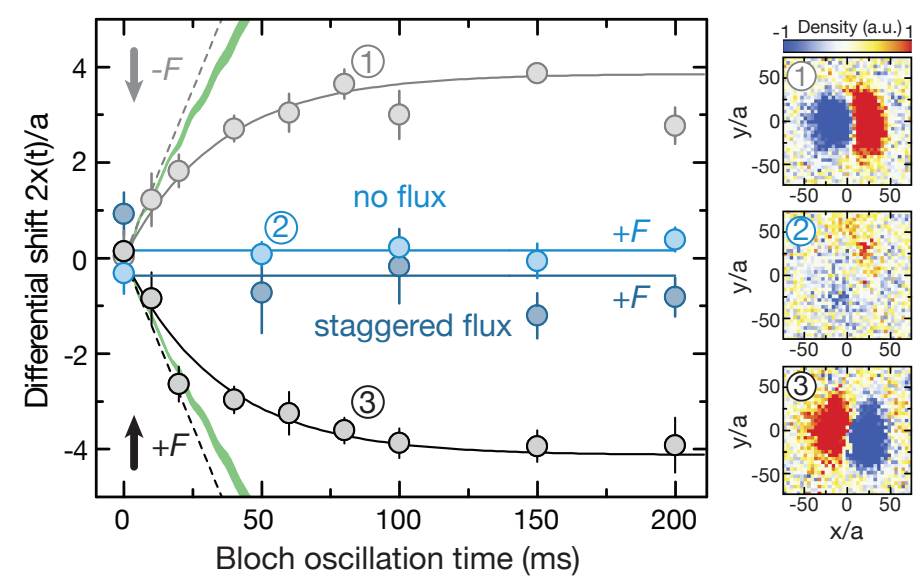

Figure 8.8: Differential COM displacement transverse to the applied force $\mathbf{F}= \pm F \hat{\mathbf{e}}_{y}$ as a function of the Bloch oscillation time. The black and gray data points show the long-time evolution of the transverse motion for opposite directions of the force $\pm F \hat{\mathbf{e}}_{y}(F a / h=38.4(8) \mathrm{Hz})$ for atoms loaded into the Hofstadter bands for $\Phi=\pi / 2$. The blue data points were taken in lattice configurations with trivial topology, $\Phi=0$ (light blue) and $\Phi=(-1)^{m} \pi / 2$ (dark blue). Each data point is an average of five datasets and the error bars show the standard error of the mean obtained from these measurements. The solid black and gray lines show a fit to our data using Eq. (8.21) taking into account the measured filling factor $\gamma(t)$ obtained from the measured band populations $\eta_{\mu}(t)$ depicted in Fig. 8.10. This provides an experimental value for the Chern number $v_{\exp }=1.05(12)$ (black) and $v_{\exp }=0.98(12)$ (gray). The blue solid lines are guides to the eye. The black and gray dashed lines depict the ideal evolution according to Eq. (8.14) using the measured initial band populations $\eta_{\mu}^{0}=\{0.63(6), 0.27(9), 0.11(4)\}$ (average of 24 individual measurements). The green shaded areas illustrate the numerical simulations based on the same initial filling factor for $\phi_{0} \in[0, \pi]$ (Sect. 7.2). The images on the right show typical experimental images obtained after subtracting the averaged in-situ images of one dataset. (Figure adapted from Ref. [73])

and gray data points in Fig. 8.8). The saturation effect is not captured anymore by the numerical simulations (green shaded are in Fig. 8.8). This suggests that additional effects associated with heating due to the modulation or due to interaction between the particles lead to additional inter-band transitions [116].

Further the COM-motion for a lattice without flux $\Phi=0$ (light blue data points in Fig. 8.8) and a staggered flux lattice $\Phi= \pm(-1)^{m} \pi / 2$ (dark blue data points in Fig. 8.8) was measured. In both cases no significant displacement of the atomic cloud transverse to the applied force was observed which is expected for lattice configurations with trivial topology where all the bands have zero Chern numbers $v_{\mu}=0$. The loading sequences were chosen similar to the one described above. For the lattice without flux the atoms were loaded within $150 \mathrm{~ms}$ into a two-dimensional staggered superlattice potential with $V_{x}=V_{y}=10(1) \mathrm{E}_{\mathrm{rs}}$ and $V_{x l}=V_{y l}=1.75(5) \mathrm{E}_{\mathrm{rl}}$. After ramping down the long lattices $V_{x l}=V_{y l}=0 \mathrm{E}_{\mathrm{rl}}$ resonant tunneling occurred along both directions 
a

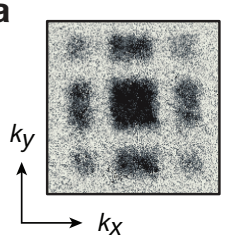

b

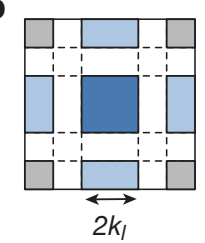

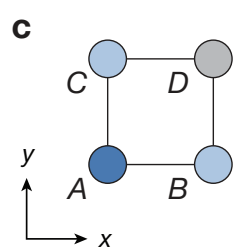

Figure 8.9: Schematic illustration of the connection between the measured atom population in the Brillouin zones and the corresponding Hofstadter bands. a Typical experimental image obtained after applying the band-mapping sequence measured after $10 \mathrm{~ms}$ TOF. $\mathbf{b}$ Brillouin zones of the static two-dimensional superlattice. $\mathrm{c}$ Labeling of the four non-equivalent sites in the unit cell of the superlattice potential. $\mathbf{d}$ Energy spectrum of the Hofstadter model for $\Phi=\pi / 2$. The color code illustrates the connection between the Brillouin zones, lattice sites and energy bands. (Figure adapted from Ref. [73])

with $J / h=75(3) \mathrm{Hz}$. For the staggered flux lattice we used the experimental setup described in Sect. 7.4 with $\omega_{r}=\omega_{b}= \pm \Delta / \hbar$. The experimental sequence was the same as described above for the uniform flux lattice.

\section{Evolution of the band populations}

Making use of independent band population measurements the band repopulation $\eta_{\mu}(t)$ and associated time-dependent filling factor $\gamma(t)$ could be quantified experimentally and included into our theoretical model by making a simple extension to Eq. (8.14).

$$
x(t)=-\frac{4 a}{\tau_{B}} v_{1} \int_{0}^{t} \gamma\left(t^{\prime}\right) \mathrm{d} t^{\prime}, \quad \gamma(t)=\left[\eta_{1}(t)-\eta_{2}(t)+\eta_{3}(t)\right] .
$$

The evolution of the band populations was measured by reversing the loading sequence as described above, similar to the momentum distribution measurements shown in Fig. 8.5. To ease the counting of the atoms in different Brillouin zones the sequence is slightly modified to separate the Brillouin zones from each other. After having switched of the modulation the system consists of a two-dimensional staggered superlattice potential with offset $\Delta+\delta_{x}$ along $x$ and $\delta_{y}$ along $y$ with $\left(\Delta+\delta_{x}\right) \gg J_{x}$ and $\delta_{y} \gg J_{y}$. Tunneling is suppressed along both directions, hence, the band occupations correspond to populations in different lattice sites: $N_{1}=N_{A}, N_{2}=N_{B}+N_{C}$ and $N_{3}=N_{D}$. By transferring the populations on different sites to even higher Bloch bands and applying a subsequent band-mapping technique we are able to determine the Hofstadter-band populations $N_{\mu}$ by counting the number of atoms in different well-separated Brillouin zones. The connection between Brillouin zones and Hofstadter bands is illustrated in Fig. 8.9. The sequence for the site-resolved detection technique is explained in Sect. 4.6. The experimental results are shown in Fig. 8.10. We find that the timescale for the repopulation between the Hofstadter bands is in agreement with the saturation timescale of the transverse atomic motion. By fitting Eq. (8.21) to our data, where $v_{1}$ is the only 


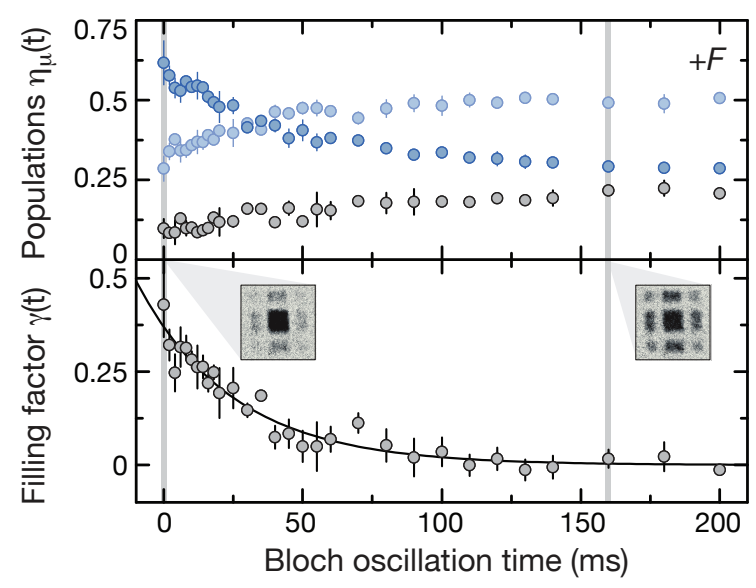

Figure 8.10: Measured band populations $\eta_{\mu}(t)$ and corresponding filling factor $\gamma(t)$. The color code used to depict the data in the upper panel illustrates the connection to the Hofstadter bands. The solid line in the lower panel is an exponential fit to our data, which was used to extract the Chern number according to Eq. (8.21). The insets in the lower panel show typical experimental images obtained after applying the band-mapping sequence. All measurements were performed for $\mathrm{Fa} / \mathrm{h}=38.4(8) \mathrm{Hz}$. Each data point is an average of two individual measurements and the error bars denote the standard deviation of the data points. (Figure adapted from Ref. [73])

free fit parameter we obtain an experimental value of the Chern number of the lowest band

$$
v_{\text {exp }}=0.99(5)
$$

This value was obtained by averaging over four independent Chern-number measurements, two for each direction of the force (see inset of Fig. 8.11). The stated uncertainty is the standard deviation obtained from these measurements. This shows that including the time-resolved band-population measurements in our analysis leads to a quantitate understanding of the transverse motion. To gain more insight into the band repopulation dynamics future studies of heating rates induced by the modulation and the interaction between the particles are necessary [116].

We also applied a complementary analysis of our data based on a more general equationof-motion that does not invoke the particle-hole symmetry (Sect. 2.3.2) and constitutes an extension of Eq. (8.13)

$$
x(t)=-\frac{4 a}{\tau_{B}}\left(v_{1} \int_{0}^{t}\left[\eta_{1}\left(t^{\prime}\right)-\eta_{3}\left(t^{\prime}\right)\right] \mathrm{d} t^{\prime}+v_{2} \int_{0}^{t}\left[\frac{\eta_{2}\left(t^{\prime}\right)}{2}-\eta_{3}\left(t^{\prime}\right)\right]\right) .
$$

This allows us to determine the Chern numbers $v_{1}$ and $v_{2}$ of the lowest two Hofstadter bands simultaneously. We interpolated the measured populations $\eta_{\mu}(t)$ depicted in Fig. 8.10 and fitted the equation to the data shown in Fig. 8.8 with the two free fit variables $v_{1}$ and $v_{2}$. We obtain $v_{1}=1.21(14), v_{2}=-2.7(5)$ (black data points) and 


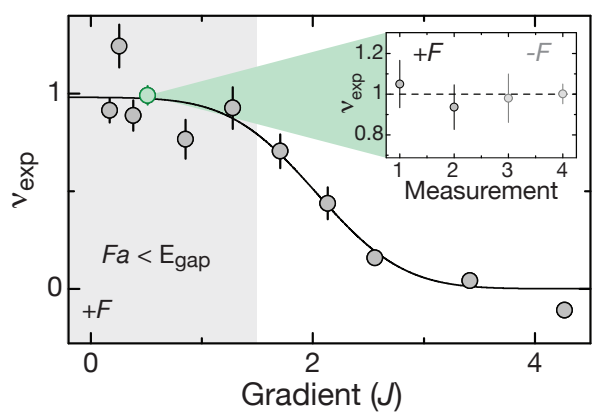

Figure 8.11: Measured Chern number as a function of the gradient strength $F a$. The experimental Chern number was determined for each value of the force by measuring the full time evolution of the differential shift $2 x(t)$ and the band populations $\eta_{\mu}(t)$, similar to the data shown in Fig. 8.8 and Fig. 8.10. The solid black line is a guide to the eye to highlight the saturation at $v_{\exp } \approx 1$ for weak forces $F a<E_{\text {gap. }}$. The green data point depicts the measured Chern number $v_{\exp }=0.99(5)$ for $F a / h=38.4(8) \mathrm{Hz}$, which was obtained from the four individual measurements displayed in the inset. Each black data point is an average of five dataset for the differential shift and two measurements for $\gamma(t)$. The vertical error bars display the uncertainty determined by the fit error of $\gamma(t)$. (Figure adapted from Ref. [73])

$v_{1}=1.04(10), v_{2}=-2.2(3)$ (gray data points), which are compatible with the theoretical values $v_{1}=+1$ and $v_{2}=-2$.

\section{Chern-number measurement for different gradients}

The measurements presented above were carried out using an optical gradient with strength $F a / h=38.4(8) \mathrm{Hz}$. This value was chosen as a compromise between two competing effects. On the one hand the force should be small in order to limit LandauZener transitions and non-linear effects. On the other hand the gradient has to be strong enough so that the displacement of the atomic cloud can be detected reliably. For gradients that are small compared to the bandgap $F a<E_{\text {gap }}$ band-repopulation timescales are similar. Therefore the measurements for small forces are more strongly affected by the repopulation since the characteristic timescales given by the Bloch period $\tau_{B}$ are naturally larger.

The dependence of the Chern-number measurement on the applied force was studied in more detail as depicted in Fig. 8.11. For gradient strengths smaller than the bandgap, $F a<E_{\text {gap }} \simeq 1.5 \mathrm{~J}$ the measured Chern number saturates to $v_{\exp } \approx 1$. This indicates that it can be extracted reliably for small forces. For larger forces $F a>E_{\text {gap }}$ the experimentally determined Chern number decreases to zero, signaling a break down of our model. We note that each data point depicted in Fig. 8.11 consists of a single Chern-number measurement (average of five individual dataset) for only one direction of the gradient, which explains the larger spread around $v_{\exp }=1$ for small forces. However, we also note that if we take into account all Chern-number measurements for gradient strengths 
smaller than the bandgap we obtain an experimental value for the Chern number $v_{\exp }=$ $0.97(4)$, where the stated uncertainty is the standard error of the mean. This value is fully compatible with $v_{\exp }=0.99(2)$, obtained from the measurements depicted in the inset of Fig. 8.11, where the stated uncertainty is the standard error of the mean. In (8.22) we have chosen to give a more conservative value of the uncertainty which is the normal standard deviation obtained from the individual measurements.

\subsubsection{Characterization of the topological phase transition}

The following measurements were again performed with $F a / h=38.4(8) \mathrm{Hz}$, which is well below the bandgap for $\delta=0$. We used the Chern-number measurements presented above to study the phase transition triggered by the staggered detuning $\delta$, which is expected to occur at $\delta=2 \mathrm{~J}$ for the Hamiltonian (8.16). The experimental results are shown in Fig. 8.12c. In agreement with the theoretical expectations we observe a decrease of the experimental Chern number to zero across the phase transition. Landau-Zener transitions during the Bloch oscillations are expected to become more important close to the phase transition point where the gaps in the energy spectrum close. However, due to our independent band-population measurements this should not affect the experimental results.

The smoothening of the transition is most likely due to experimental uncertainties in the resonance condition. Possible drifts and fluctuations were recorded by calibrating the resonance frequency several times over the course of one day and evaluating the corresponding standard deviation $\sigma_{\delta}=0.4 \mathrm{~J}$. This was included in the model by convoluting the ideal transition at $\delta=2 J$ with a normal distribution at the mean detuning $\delta$ and the standard deviation $\sigma_{\delta}$ (green solid line in Fig. 8.12c). This qualitatively agrees with the data.

In Figure 8.12a,b numerical calculations of the Berry curvature are shown for two different values of the detuning, one on either side of the topological phase transition. The calculations were performed according to the method described in Ref. [99] and Sect. 2.4. The Berry curvature is a local property that is defined for each state $\left|u_{\mu}(\mathbf{k})\right\rangle$ at momentum $\mathbf{k}$ within the FBZ according to Eq. (2.46). For the topologically non-trivial band the sign of $\Omega_{1}(\mathbf{k})$ is uniform across the first Brillouin zone such that the integral over the full FBZ does not vanish; in particular for the lowest Hofstadter band with $\Phi=\pi / 2$ it results in $v_{1}=+1$ (Fig. 8.12a). For the topologically trivial situation, however, we see that the Berry curvature changes sign within the FBZ. This leads to a vanishing Chern number $v_{1}=0$ for the lowest band of the Hofstadter-like lattice with $\delta>2 J$ when integrating the curvature over the whole FBZ (Fig. 8.12a). Note that similar to the energy spectrum the Berry curvature distribution might be shifted in momentum space depending on the choice of gauge. 

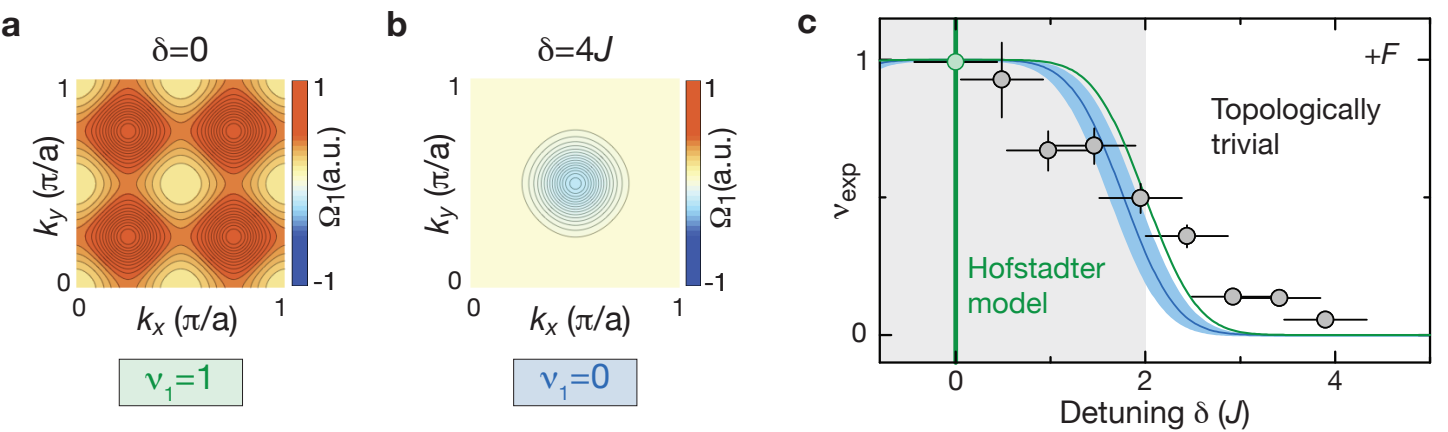

Figure 8.12: Berry curvature and Chern number as a function of the staggered detuning $\delta$. a Berry curvature $\Omega_{1}(\mathbf{k})$ of the lowest Hofstadter band $(\delta=0)$. It exhibits only positive contributions which leads to a non-zero Chern number $v_{1}=1$. $\mathbf{b}$ Berry curvature of the lowest band of the Hofstadter-like lattice for $\delta=4 \mathrm{~J}$. Contrary to the topologically non-trivial case the Berry curvature exhibits positive and negative values resulting in $v_{1}=0$. c Measured Chern number versus detuning for $\mathrm{Fa} / \mathrm{h}=38.4(8) \mathrm{Hz}$. For each value of the detuning the differential shift $2 x(t)$ was measured at four different times $t=(20,50,100,150) \mathrm{ms}$, averaged over five dataset. The corresponding band populations $\eta_{\mu}(t)$ were measured as depicted in Fig. 8.10, averaged over two individual measurements. To extract the Chern number we fit Eq. (8.21) using the measured filling factor $\gamma(t) ; v_{1}$ was the only free fit variable. The green data point is the averaged value of the Chern number $v_{\exp }=0.99(5)$ for $\delta=0$ as given in (8.22). The topological phase transitions occurs at $\delta=2 J$ for Hamiltonian (8.16). As explained in the main text, the transition is smoothened due to experimental uncertainties $\sigma_{\delta}=0.4 \mathrm{~J}$ (horizontal error bars) in the determination of the resonance condition (green solid curve). The blue shaded area illustrates the range of transition points $\delta \approx 1.77(14) \mathrm{J}$ governed by the Hamiltonian (8.25), which includes higher order corrections due to the initial phase of the modulation $\phi_{0}$ (see Fig. 8.14). The vertical error bars show the uncertainty of the experimental value for the Chern number resulting from the fit errors related to $\gamma(t)$. (Figure adapted from Ref. [73])

\section{Higher-order corrections}

So far we have neglected the corrections that arise due to the initial phase of the modulation $\phi_{0}$. This phase can lead to small modifications of the coupling strength $J$ along $y$ as discussed in Sect. 7.2. For our experimental parameters $V_{0} /(\hbar \omega)=0.58(2)$ the initial phase $\phi_{0}$ can cause an inhomogeneity in the coupling along $y$ up to $30 \%$ (Fig. 7.4b). We further incorporate the corrections given in Eq. (7.15) into the derivation of the effective Hamiltonian. The modified Schrödinger equation now reads

$$
\begin{aligned}
E \Psi_{m, n}= & -J\left[\mathrm{e}^{i n \pi / 2} \Psi_{m+1, n}+\mathrm{e}^{-i n \pi / 2} \Psi_{m-1, n}\right] \\
& -J\left[\left(1+f_{m, n}\right) \Psi_{m, n+1}+\left(1+f_{m, n-1}\right) \Psi_{m, n-1}\right] .
\end{aligned}
$$

Using the same ansatz for the wave function as given above, Eq. (8.3), we obtain the following modified Hamiltonian $\hat{H} \rightarrow \hat{H}_{\mathrm{c}}$ (see also Eq. (8.16)), 

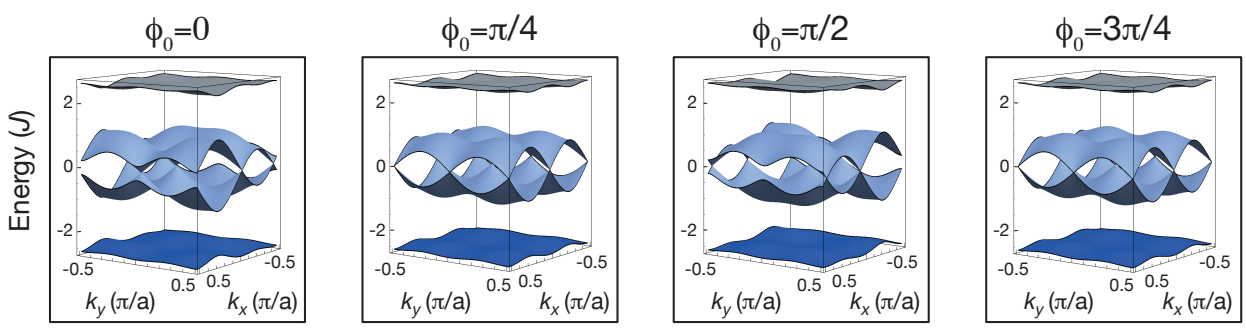

Figure 8.13: Energy spectrum of the Hofstadter model for $\delta=0$ in the presence of small higher-order corrections. The corrections are determined by the phase of the modulation $\phi_{0}$, which can lead to inhomogeneous couplings along $y$. For $\phi_{0}=0$ and $\phi_{0}=\pi / 2$ the inhomogeneity is maximal, with $J_{\min }=0.69(2) J$ and $J_{\max }=J_{y}$. For $\phi_{0}= \pm \pi / 4$ the coupling is homogeneous with $J_{\min }=J_{\max }=0.84(1) J$. The topology of the bands is robust against these corrections independent of the value of $\phi_{0}$.

$$
\hat{H}_{\mathrm{c}}=-2 J\left(\begin{array}{cccc}
\delta /(2 J) & i \sin \left(k_{x} a\right) & -\sin \left(k_{y} a\right)+h_{1} & 0 \\
-i \sin \left(k_{x} a\right) & 0 & 0 & \cos \left(k_{y} a\right)+h_{2}^{*} \\
-\sin \left(k_{y} a\right)+h_{1}^{*} & 0 & 0 & \cos \left(k_{x} a\right) \\
0 & \cos \left(k_{y} a\right)+h_{2} & \cos \left(k_{x} a\right) & -\delta /(2 J)
\end{array}\right) \text {, }
$$

where the small higher-order corrections are determined by $\phi_{0}$ and $V_{0} /(\hbar \omega)$

$$
\begin{aligned}
& h_{1}=+\frac{1}{2}\left(\frac{V_{0}}{\hbar \omega}\right)^{2}\left[\sin \left(k_{y} a\right)+i \cos \left(2 \phi_{0}\right) \cos \left(k_{y} a\right)\right] \\
& h_{2}=-\frac{1}{2}\left(\frac{V_{0}}{\hbar \omega}\right)^{2}\left[\cos \left(k_{y} a\right)-i \cos \left(2 \phi_{0}\right) \sin \left(k_{y} a\right)\right] .
\end{aligned}
$$

Figure 8.13 illustrates the energy spectrum for the Hofstadter model $\delta=0$ for four different values of the phase. The largest deviations are obtained for $\phi_{0}=0$ and $\phi_{0}=$ $\pi / 2$, where the inhomogeneity in the coupling is maximal. For $\phi_{0}=(k \pm 1 / 4) \pi$, with $k$ integer, the coupling along $y$ is homogeneous and the spectrum is very similar to the ideal Hofstadter bands. In this section we studied the topology of the bands across the phase transition, which ideally occurs at $\delta=2 J$. Using Hamiltonian (8.25) we find that the phase transition point depends weakly on the value of the phase $\phi_{0}$. The corrections mainly shift the transition point to lower detunings $\delta \approx 1.77 \mathrm{~J}$ (Fig. 8.14). The blue shaded area in Fig. 8.12c illustrates the different transition points for $\delta=1.77(14)$, where we defined the transition region in the interval $E_{\text {gap }} \in[0,0.1] J$. We observe that the corrections are within our experimental error bars. 


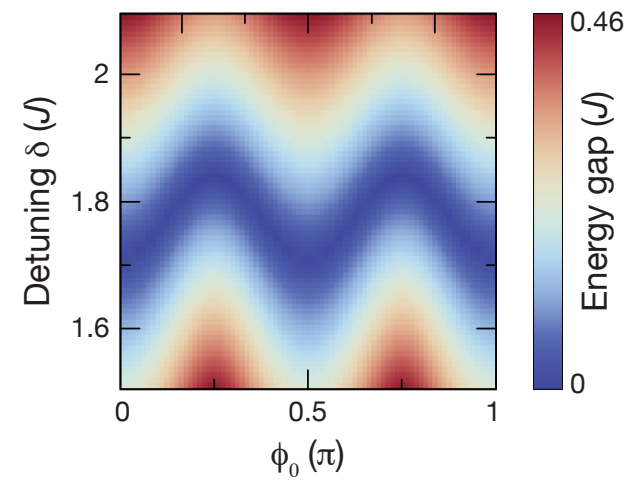

Figure 8.14: Numerical calculations of the energy gap $E_{\text {gap }}$ and the topological phase transition point using Hamiltonian (8.25). Energy gap as a function of the phase $\phi_{0}$ and the staggered detuning $\delta$. In general the gap-closing point appears to be shifted to $\delta \approx 1.77 \mathrm{~J}$ compared to the ideal value of $\delta=2 J$. 


\section{Chapter 9}

\section{Conclusions and Outlook}

The main topic of this thesis was the implementation of artificial magnetic fields with ultracold atoms in optical lattices. A new experimental technique based on laser-assisted tunneling was successfully demonstrated, that led to the generation of synthetic magnetic fields on the order of one flux quantum per lattice unit cell. In chapter 5 it was shown that laser-assisted tunneling in a staggered superlattice potential in its simplest form, where tunneling on each bond is triggered simultaneously with the same pair of laser beams, naturally leads to a staggered flux distribution with zero mean. In these systems the momentum distribution of the ground state was studied in time-of-flight experiments. The corresponding experimental results were shown to be in agreement with a theoretical description in terms of the effective time-independent Floquet Hamiltonian. The underlying theoretical framework was summarized in chapter 3, which also included a detailed discussion of the full-time dynamics that occur within one driving period. Experimental signatures of these additional dynamics were observed, supporting its relevance for experimental realizations. The structure of the artificial magnetic field was further examined on the level of isolated four-site square plaquettes, which enabled a direct experimental determination of the strength of the artificial flux per unit cell through the observation of local cyclotron orbits. The experimental results were shown to be in agreement with the theoretically predicted full-time dynamics.

In chapter 6 and 7 two modifications of the initial laser-assisted tunneling scheme, that was presented in chapter 5 , were studied, which led to the realization of a uniform flux configuration. In the first scheme (chapter 6) the flux was rectified by replacing the staggered potential with a linear one that was realized with a magnetic field gradient. By including an additional pseudo-spin degree of freedom into the same system a Hamiltonian was implemented, that underlies the quantum spin Hall effect. A first signature of this was the observation of the spin Hall effect in an optical lattice using cyclotron-orbit measurements. The second scheme (chapter 7) was again based on a staggered superlattice potential. This time the laser-beam configuration for the laser-assisted tunneling scheme was modified such that tunneling was restored only on every other bond with 
a single pair of beams. By making use of two spatially separated pairs, tunneling was independently restored on the two kinds of bonds, thus enabling flux rectification. This scheme only relies on optical potentials which increases the experimental flexibility in comparison with the use of magnetic field gradients and was the starting point for the achievement of an adiabatic loading of the atoms into the topological bands of the Hofstadter model.

In chapter 8 the new all-optical scheme was eventually employed to determine the topological invariant, the Chern number, of the lowest Hofstadter band. In these experiments the transverse deflection of an atomic cloud was observed as a response to an applied force through in-situ imaging. For a filled energy band, the center-of-mass evolution of the cloud is expected to be proportional to the Chern number of the corresponding band. In our experiment this has been realized with bosonic atoms populating the lowest Hofstadter band homogeneously both through interactions and finite temperature. This experiment constitutes the first Chern-number measurement in a non-electronic system. Furthermore the techniques which were developed in this work with bosonic atoms are applicable to a wide range of other non-electronic systems [65, 82, 202].

\section{Outlook}

In the context of this thesis interactions between the atoms were typically very small and could be neglected compared to the other energy scales in the system. For the Chernnumber measurement presented in chapter 8 , however, the situation might be different. In the presence of a uniform magnetic field the lowest tight-binding band splits into several subbands, which naturally decreases the relevant energy scales in the system. For our experimental parameters the interaction energies are on the order of the energy gap between the lowest two Hofstadter bands. This could be a reason for the repopulation between the Hofstadter bands which was observed during the dynamics. The Floquet energy in periodically driven systems is only defined up to integer multiples of the modulation frequency $\omega$ (chapter 3). In many cases the theoretical discussion is restricted to one Floquet band only. Scattering between the particles, however, can lead to a coupling between different Floquet modes, which may in turn lead to a transfer of the atoms into higher bands under the absorption of one or several "Floquet-photons" with energy $\hbar \omega$. Time-dependent Hamiltonians without interactions were extensively studied in the literature, however, a detailed understanding of scattering effects just started to be put forward [116, 203-206]. Thermodynamic properties of periodically driven quantum systems were studied in Refs. [207-209]. Future experiments could reveal new insights by measuring heating rates in the presence of a periodic modulation for different parameter regimes. In 3D lattice systems, e.g. stability islands are predicted where the coupling between different Floquet modes is suppressed [116]. Using Feshbach resonances [45] heating rates could be further studied as a function of the interaction strength. 
The realization of artificial magnetic fields using laser-assisted tunneling is commonly described in terms of the effective time-independent Floquet Hamiltonian. In chapter 3 the corresponding micro-motion was discussed using the example of one-dimensional driven lattices. This micro-motion can be very different for driving schemes that result in the same effective flux distribution but are based on different experimental setups [66]. This suggests that some schemes might be more susceptible to modulation-induced heating than others. In a theoretical study about the orbital-driven melting of a bosonic Mott insulator [210] it was found that a periodic shaking of the lattice potential back and forth limits the transfer into higher bands compared to other driving schemes that rely on on-site modulations. Future experiments could clarify related questions.

Once a better understanding of interaction effects and modulation-induced heating is achieved, the inclusion of interactions could lead to the observation of strongly correlated states in flat topological bands, such as fractional Chern insulators [13, 27]. The Hofstadter bands realized with our current setup (chapter 7-8) with flux $\Phi=2 \pi \alpha=$ $\pi / 2$ exhibit a flatness ratio of $E_{\mathrm{gap}} / E_{\mathrm{bw}} \simeq 7$ and are therefore promising candidates to realize such systems. For bosonic atoms there exists a bosonic Laughlin state for filling $1 / 2$, which means that the number of flux quanta $N_{\Phi}=2 N$ is twice the number of particles $N$. However, the regime of strong interactions was initially only studied for $\alpha \ll 1$ since large field strengths, with $\alpha$ on the order of one, were not achievable in early experiments. For larger fluxes, effects related to the presence of the lattice become important. It has been shown that the ground state of hard-core bosons in the Hofstadter bands has a very large overlap with the Laughlin wave function for fluxes $\alpha<0.3$ [211]. In this regime the lowest Hofstadter band is very flat and the Laughlin wave function is a good description. Experimental evidence of this state might be even observable by measuring the momentum distribution of the atoms after time-of-flight [211]. This study was extended to the regime of finite interactions in Ref. [212], where in addition the topological nature of the ground state was explored in regimes where the overlap with the Laughlin states is reduced. Filling factors different from $1 / 2$ for bosonic systems were studied in Ref. [213] also beyond a simple comparison to the continuum limit [214].

A first step regarding the experimental realization of strongly-correlated states in the presence of artificial gauge fields might be achieved with optical ladder systems. This quasi one-dimensional geometry is the minimal lattice system where the effect of artificial gauge fields can be studied. Previously, this has led to the observation of a transition between a phase with Meissner-like chiral currents and a vortex phase [72]. Several theoretical studies discussed the different strongly-correlated phases that occur in these systems [215-222]. In combination with recently developed high-resolution detection techniques $[50,163]$ the chiral currents could be measured in a spatially-resolved manner [223]. Possibly this might also allow for an experimental study of the connection between the chiral Meissner currents and the edge states of an integer quantum Hall insulator [224, 225]. 


\section{Appendix A}

\section{Magnetic translation operators}

\section{Derivation}

The derivation of the phases $\theta_{m, n}^{i}, i=\{x, y\}$, in the definition of the magnetic translation operators, Eq. (2.8), follows closely the calculations presented in Ref. [90]. We require that all commutators between the MTOs and the Hamiltonian $\hat{H}$ as defined in Eqs. (2.6)(2.7) vanish, $\left[\hat{T}_{x}^{M}, \hat{H}\right]=0$ and $\left[\hat{T}_{y}^{M}, \hat{H}\right]=0$. This imposes several constraints on the phases

$$
\begin{gathered}
{\left[\hat{T}_{x}^{M}, \hat{T}_{x}\right]=\mathrm{e}^{i\left(\phi_{m, n}^{x}+\theta_{m+1, n}^{x}\right)}\left[1-\mathrm{e}^{i\left(\theta_{m, n}^{x}+\phi_{m+1, n}^{x}-\phi_{m, n}^{x}-\theta_{m+1, n}^{x}\right)} \stackrel{!}{=} 0\right.} \\
\Rightarrow \Delta_{x} \theta_{m, n}^{x}=\Delta_{x} \phi_{m, n}^{x}, \\
{\left[\hat{T}_{x}^{M}, \hat{T}_{y}\right]=\mathrm{e}^{i\left(\phi_{m, n}^{y}+\theta_{m, n+1}^{x}\right)}\left[1-\mathrm{e}^{i\left(\theta_{m, n}^{x}+\phi_{m+1, n}^{y}-\phi_{m, n}^{y}-\theta_{m, n+1}^{x}\right)}\right] \stackrel{!}{=} 0} \\
\Rightarrow \Delta_{y} \theta_{m, n}^{x}=\Delta_{x} \phi_{m, n}^{y}=\Delta_{y} \phi_{m, n}^{x}+\Phi_{m, n},
\end{gathered}
$$

where we have used the definition for the derivative on a discrete lattice as given in Eq. (5.11), together with Eq. (2.10). The remaining commutator $\left[\hat{T}_{y}^{M}, \hat{H}\right]=0$ imposes analog constraints on the phases

$$
\begin{aligned}
{\left[\hat{T}_{y}^{M}, \hat{T}_{x}\right] } & =\mathrm{e}^{i\left(\phi_{m, n}^{x}+\theta_{m+1, n}^{y}\right)}\left[1-\mathrm{e}^{i\left(\theta_{m, n}^{y}+\phi_{m, n+1}^{x}-\phi_{m, n}^{x}-\theta_{m+1, n}^{y}\right)}\right] \stackrel{!}{=} 0 \\
& \Rightarrow \Delta_{x} \theta_{m, n}^{y}=\Delta_{y} \phi_{m, n}^{x}=\Delta_{x} \phi_{m, n}^{y}-\Phi_{m, n}
\end{aligned}
$$




$$
\begin{gathered}
{\left[\hat{T}_{y}^{M}, \hat{T}_{y}\right]=\mathrm{e}^{i\left(\phi_{m, n}^{y}+\theta_{m, n+1}^{y}\right)}\left[1-\mathrm{e}^{i\left(\theta_{m, n}^{y}+\phi_{m, n+1}^{y}-\phi_{m, n}^{y}-\theta_{m, n+1}^{y}\right)}\right] \stackrel{!}{=} 0} \\
\Rightarrow \Delta_{y} \theta_{m, n}^{y}=\Delta_{y} \phi_{m, n}^{y} .
\end{gathered}
$$

The conditions imposed by equations A.1-A.4 can be solved by

$$
\theta_{m, n}^{x}=\phi_{m, n}^{x}+\Phi_{m, n} n \quad \theta_{m, n}^{y}=\phi_{m, n}^{y}-\Phi_{m, n} m,
$$

which matches the definition in Eq. (2.9) of Sect. 2.2. 


\section{Appendix B}

\section{Propagation operator}

For time-periodic Hamiltonians $\hat{H}(t+T)=\hat{H}(t)$ Floquet's theorem states that the solution of the time-dependent Schrödinger equation

$$
i \hbar \frac{\partial}{\partial t} \hat{U}(t)=\hat{H}(t) \hat{U}(t)
$$

can be written in the following form

$$
\hat{U}(t)=\hat{P}(t) \mathrm{e}^{-\frac{i}{\hbar} \hat{H}_{F} t},
$$

where the propagation operator $\hat{P}(t+T)=\hat{P}(t)$ is a unitary operator which is periodic in time $[117,123]$ and $\hat{H}_{F}$ is a time-independent hermitian operator. Proving the timeperiodicity of $\hat{P}(t)$ is rather straightforward. If $\hat{U}(t)$ is a solution of the time-dependent Schrödinger equation so is $\hat{V}(t)=\hat{U}(t+T)$, with $\hat{V}(0)=\hat{U}(T)$. By applying the operator $\hat{U}(T)$ from the right side to the Schrödinger equation one obtains

$$
i \hbar \underbrace{\partial_{t} \hat{U}(t) \hat{U}(T)}_{\partial_{t} \hat{W}(t)}=\hat{H}(t) \underbrace{\hat{U}(t) \hat{U}(T)}_{\hat{W}(t)},
$$

which means that $\hat{W}(t)$ is also a solution to the Schrödinger equation with $\hat{W}(0)=\hat{U}(T)$. Since the solution to the Schrödinger equation is unique it follows that $\hat{V}(t)=\hat{W}(t)$ or $\hat{U}(t+T)=\hat{U}(t) \hat{U}(T)$ and moreover

$$
\hat{P}(t+T)=\hat{U}(t+T) \mathrm{e}^{\frac{i}{\hbar} \hat{H}_{F}(t+T)}=U(t) \underbrace{\hat{U}(T) \mathrm{e}^{\frac{i}{\hbar} \hat{H}_{F}(t+T)}}_{\mathrm{e}^{-\frac{i}{\hbar} \hat{H}_{F} T} \mathrm{e}^{\frac{i}{\hbar} \hat{H}_{F}(t+T)}=\mathrm{e}^{\frac{i}{\hbar}} \hat{H}_{F} t}=\hat{U}(t) \mathrm{e}^{\frac{i}{\hbar} \hat{H}_{F} t}=\hat{P}(t) .
$$




\section{Appendix C}

\section{Staggered flux distribution}

\section{Experimental gauge}

It was shown in Sect. 5.2 that the momentum distribution measured in cold-atom experiments using expansion imaging is a gauge-dependent quantity. Because of that the effective Floquet Hamiltonian needs to be derived in the experimental gauge which is determined by the laser-beam configuration chosen for the realization of the on-site modulation. In Sect. 5.4 it was mentioned that the measured momentum distributions are independent of the initial phase of the driving $\phi_{0}$. This was verified numerically by exact diagonalization. An equivalent result can be obtained analytically by solving the Schrödinger equation associated with the effective Floquet Hamiltonian (5.28) as will be demonstrated in the following.

The magnetic translation operators cannot be found easily by direct application of Eq. (2.9), which was derived in Appendix A. However, using the knowledge about the dimensions of the magnetic unit cell $A_{M U}=2 a \times 1 a$ they can be derived following the general strategy presented in Appendix A. The Peierls phase-factors realized in the experiment (5.32) are given by

$$
\phi_{m, n}= \begin{cases}-\frac{\pi}{2}(m-n+3 / 2)-\phi_{0}, & \text { for } m \text { odd } \\ +\frac{\pi}{2}(m-n+7 / 2)+\phi_{0}, & \text { for } m \text { even }\end{cases}
$$

We find that the MTOs can be written in the following form

$$
\begin{aligned}
& \hat{M}_{x}^{2}=\sum_{m, n} \hat{a}_{m+2, n}^{\dagger} \hat{a}_{m, n} \begin{cases}e^{i 4 \pi \alpha}, & \text { for } m \text { odd } \\
1, & \text { for } m \text { even }\end{cases} \\
& \hat{M}_{y}^{1}=\sum_{m, n} \hat{a}_{m, n+1}^{\dagger} \hat{a}_{m, n} \begin{cases}e^{-i 2 \pi \alpha}, & \text { for } m \text { odd } \\
1, & \text { for } m \text { even }\end{cases}
\end{aligned}
$$


Fulfilling Bloch's theorem defined in Eq. (2.18) we can make the following ansatz for the wave function

$$
\Psi_{m, n}=\mathrm{e}^{i k_{x} m a} \mathrm{e}^{i k_{y} n a}\left\{\begin{array}{ll}
\psi_{0} \mathrm{e}^{-i 2 \pi \alpha(m-n)}, & \text { for } m \text { odd } \\
\psi_{e}, & \text { for } m \text { even }
\end{array},\right.
$$

where $k_{x}$ and $k_{y}$ are defined within the first magnetic Brillouin zone, $-\pi /(2 a) \leq k_{x}<$ $\pi /(2 a)$ and $-\pi / a \leq k_{y}<\pi / a$. Plugging this ansatz into the Schrödingern equation (5.6) results in a two-dimensional eigenvalue equation

$$
\begin{gathered}
\hat{H}\left(\begin{array}{l}
\psi_{e} \\
\psi_{0}
\end{array}\right)=E(\mathbf{k})\left(\begin{array}{c}
\psi_{e} \\
\psi_{0}
\end{array}\right), \\
\hat{H}=\left(\begin{array}{cc}
-2 J \cos \left(k_{y} a\right) & -K\left(\mathrm{e}^{i\left(-2 \pi \alpha+\phi_{\mathrm{even}}+k_{x} a\right)}+\mathrm{e}^{-i\left(\phi_{\mathrm{odd}}+k_{x} a\right)}\right) \\
-K\left(\mathrm{e}^{-i\left(-2 \pi \alpha+\phi_{\mathrm{even}}+k_{x} a\right)}+\mathrm{e}^{i\left(\phi_{\mathrm{odd}}+k_{x} a\right)}\right) & -2 J \cos \left(k_{y} a+2 \pi \alpha\right)
\end{array}\right),
\end{gathered}
$$

with $\phi_{\text {even }}=7 \pi / 4+\phi_{0}$ and $\phi_{\text {odd }}=-3 \pi / 4-\phi_{0}$. The dispersion relation determined by (C.3) does not depend on $\phi_{0}$, which means that the momentum distributions neither depend on it. Therefore we choose without loss of generality $\phi_{0}=-3 \pi / 4$.

Simplified form of the gauge field as defined in Eq. (5.37): In this section we are going to present the solutions to the Schrödinger equation associated with the Hamiltonian (5.37) for a more general form of the gauge field $\phi_{m, n}=2 \pi \alpha(m+n)(-1)^{(m+1)}$. The MTOs can be written in the following form

$$
\begin{aligned}
& \hat{M}_{x}^{2}=\sum_{m, n} \hat{a}_{m+2, n}^{+} \hat{a}_{m, n}\left\{\begin{array}{ll}
e^{-i 4 \pi \alpha}, & \text { for } m \text { odd } \\
1, & \text { for } m \text { even }
\end{array},\right. \\
& \hat{M}_{y}^{1}=\sum_{m, n} \hat{a}_{m, n+1}^{+} \hat{a}_{m, n}\left\{\begin{array}{ll}
e^{-2 i \pi \alpha}, & \text { for } m \text { odd } \\
1, & \text { for } m \text { even }
\end{array},\right.
\end{aligned}
$$

such that the ansatz for the wave function can be written in the following form

$$
\Psi_{m, n}=\mathrm{e}^{i k_{x} m a} \mathrm{e}^{i k_{y} n a}\left\{\begin{array}{ll}
\psi_{0} \mathrm{e}^{i 2 \pi \alpha(m+n)}, & \text { for } m \text { odd } \\
\psi_{e}, & \text { for } m \text { even }
\end{array},\right.
$$

where $k_{x}$ and $k_{y}$ are defined within the FBZ defined above. Inserting this ansatz into the Schrödinger equation (5.6) leads to the two-dimensional eigenvalue equation

$$
\left(\begin{array}{cc}
-2 J \cos \left(k_{y} a\right) & -K\left(\mathrm{e}^{i 2 \pi \alpha} \mathrm{e}^{i k_{x} a}+\mathrm{e}^{-i k_{x} a}\right) \\
-K\left(\mathrm{e}^{-i 2 \pi \alpha} \mathrm{e}^{-i k_{x} a}+\mathrm{e}^{i k_{x} a}\right) & -2 J \cos \left(k_{y} a+2 \pi \alpha\right)
\end{array}\right)\left(\begin{array}{c}
\psi_{e} \\
\psi_{0}
\end{array}\right)=E(\mathbf{k})\left(\begin{array}{c}
\psi_{e} \\
\psi_{0}
\end{array}\right) .
$$

For $\alpha=1 / 4$ we recover the solutions presented in Sect. 5.5. 


\section{References}

[1] P. W. Anderson. Basic Notions of Condensed Matter Physics. Westview Press, Boulder, CO (1997). Cited on p. 1.

[2] K. von Klitzing, G. Dorda, and M. Pepper. New Method for High-Accuracy Determination of the Fine-Structure Constant Based on Quantized Hall Resistance. Phys. Rev. Lett. 45, 494-497 (1980). Cited on p. 1, 19.

[3] K. von Klitzing. The quantized Hall effect. Rev. Mod. Phys. 58, 519-531 (1986). Cited on p. 1.

[4] M. Z. Hasan and C. L. Kane. Colloquium: Topological insulators. Rev. Mod. Phys. 82, 3045-3067 (2010). Cited on p. 1, 2.

[5] J. E. Moore. The birth of topological insulators. Nature 464, 194-198 (2010). Cited on p. 1.

[6] X.-L. Qi and S.-C. Zhang. Topological insulators and superconductors. Rev. Mod. Phys. 83, 1057-1110 (2011). Cited on p. 1, 2.

[7] R. B. Laughlin. Quantized Hall conductivity in two dimensions. Phys. Rev. B 23, 5632-5633 (1981). Cited on p. 1.

[8] D. J. Thouless, M. Kohmoto, M. P. Nightingale, and M. den Nijs. Quantized Hall Conductance in a Two-Dimensional Periodic Potential. Phys. Rev. Lett. 49, 405-408 (1982). Cited on p. 1, 2, 19, 21.

[9] J. B. Listing. Vorstudien zur Topologie. Vanderhoeck und Ruprecht, Göttingen (1848). Cited on p. 1.

[10] Y. Hatsugai. Chern number and edge states in the integer quantum Hall effect. Phys. Rev. Lett. 71, 3697-3700 (1993). Cited on p. 2, 20, 128.

[11] Y. Hatsugai. Edge states in the integer quantum Hall effect and the Riemann surface of the Bloch function. Phys. Rev. B 48, 11851-11862 (1993). Cited on p. 2, 20, 128. 
[12] X.-L. Qi, Y.-S. Wu, and S.-C. Zhang. General theorem relating the bulk topological number to edge states in two-dimensional insulators. Phys. Rev. B 74, 045125 (2006). Cited on p. 2, 20.

[13] E. J. Bergholtz and Z. Liu. Topological flat band models and fractional Chern insulators. Int. J. Mod. Phys. B 27, 1330017 (2013). Cited on p. 2, 149.

[14] F. D. M. Haldane. Model for a Quantum Hall Effect without Landau Levels: CondensedMatter Realization of the "Parity Anomaly". Phys. Rev. Lett. 61, 2015-2018 (1988). Cited on p. 2, 3.

[15] C. L. Kane and E. J. Mele. Quantum Spin Hall Effect in Graphene. Phys. Rev. Lett. 95, 226801 (2005). Cited on p. 2, 5, 93, 94.

[16] B. A. Bernevig and S.-C. Zhang. Quantum Spin Hall Effect. Phys. Rev. Lett. 96, 106802 (2006). Cited on p. 2, 5, 93, 94.

[17] M. König, S. Wiedmann, C. Brüne, A. Roth, H. Buhmann, L. W. Molenkamp, X.-L. Qi, and S.-C. Zhang. Quantum Spin Hall Insulator State in HgTe Quantum Wells. Science 318, 766-770 (2007). Cited on p. 2.

[18] M. König, H. Buhmann, L. W. Molenkamp, T. Hughes, C.-X. Liu, X.-L. Qi, and S.-C. Zhang. The Quantum Spin Hall Effect: Theory and Experiment. J. Phys. Soc. Jpn. 77, 031007 (2008). Cited on p. 2.

[19] A. Roth, C. Brüne, H. Buhmann, L. W. Molenkamp, J. Maciejko, X.-L. Qi, and S.-C. Zhang. Nonlocal Transport in the Quantum Spin Hall State. Science 325, 294-297 (2009). Cited on p. 2.

[20] L. Fu, C. L. Kane, and E. J. Mele. Topological Insulators in Three Dimensions. Phys. Rev. Lett. 98, 106803 (2007). Cited on p. 2.

[21] J. E. Moore and L. Balents. Topological invariants of time-reversal-invariant band structures. Phys. Rev. B 75, 121306 (2007). Cited on p. 2.

[22] R. Roy. Topological phases and the quantum spin Hall effect in three dimensions. Phys. Rev. B 79, 195322 (2009). Cited on p. 2.

[23] D. Hsieh, D. Qian, L. Wray, Y. Xia, Y. S. Hor, R. J. Cava, and M. Z. Hasan. A topological Dirac insulator in a quantum spin Hall phase. Nature 452, 970-974 (2008). Cited on p. 2.

[24] Y. Xia, D. Qian, D. Hsieh, L. Wray, A. Pal, H. Lin, A. Bansil, D. Grauer, Y. S. Hor, R. J. Cava, and M. Z. Hasan. Observation of a large-gap topological-insulator class with a single Dirac cone on the surface. Nature Phys. 5, 398-402 (2009). Cited on p. 2. 
[25] D. C. Tsui, H. L. Stormer, and A. C. Gossard. Two-Dimensional Magnetotransport in the Extreme Quantum Limit. Phys. Rev. Lett. 48, 1559-1562 (1982). Cited on p. 2.

[26] R. B. Laughlin. Anomalous Quantum Hall Effect: An Incompressible Quantum Fluid with Fractionally Charged Excitations. Phys. Rev. Lett. 50, 1395-1398 (1983). Cited on p. 2.

[27] S. A. Parameswaran, R. Roy, and S. L. Sondhi. Fractional quantum Hall physics in topological flat bands. C. R. Phys. 14, 816-839 (2013). Cited on p. 2, 128, 149.

[28] C.-Z. Chang, J. Zhang, X. Feng, J. Shen, Z. Zhang, M. Guo, K. Li, Y. Ou, P. Wei, L.L. Wang, Z.-Q. Ji, Y. Feng, S. Ji, X. Chen, J. Jia, X. Dai, Z. Fang, S.-C. Zhang, K. He, Y. Wang, L. Lu, X.-C. Ma, and Q.-K. Xue. Experimental Observation of the Quantum Anomalous Hall Effect in a Magnetic Topological Insulator. Science 340, 167-170 (2013). Cited on p. 3.

[29] I. Bloch, J. Dalibard, and W. Zwerger. Many-body physics with ultracold gases. Rev. Mod. Phys. 80, 885-964 (2008). Cited on p. 3, 37, 47, 49, 63, 65.

[30] I. Bloch, J. Dalibard, and S. Nascimbène. Quantum simulations with ultracold quantum gases. Nature Phys. 8, 267-276 (2012). Cited on p. 3.

[31] K. I. Petsas, A. B. Coates, and G. Grynberg. Crystallography of optical lattices. Phys. Rev. A 50, 5173-5189 (1994). Cited on p. 3.

[32] M. Greiner, I. Bloch, O. Mandel, T. Hänsch, and T. Esslinger. Exploring Phase Coherence in a 2D Lattice of Bose-Einstein Condensates. Phys. Rev. Lett. 87, 160405 (2001). Cited on p. 3, 55.

[33] J. Sebby-Strabley, M. Anderlini, P. S. Jessen, and J. V. Porto. Lattice of double wells for manipulating pairs of cold atoms. Phys. Rev. A 73, 033605 (2006). Cited on p. 3, 55,81 .

[34] S. Fölling, S. Trotzky, P. Cheinet, M. Feld, R. Saers, A. Widera, T. Müller, and I. Bloch. Direct observation of second-order atom tunnelling. Nature 448, 1029-32 (2007). Cited on p. 3, 55, 80, 81.

[35] C. Becker, P. Soltan-Panahi, J. Kronjäger, S. Dörscher, K. Bongs, and K. Sengstock. Ultracold quantum gases in triangular optical lattices. New. J. Phys. 12, 065025 (2010). Cited on p. 3.

[36] L. Tarruell, D. Greif, T. Uehlinger, G. Jotzu, and T. Esslinger. Creating, moving and merging Dirac points with a Fermi gas in a tunable honeycomb lattice. Nature 483, 302-305 (2012). Cited on p. 3. 
[37] G.-B. Jo, J. Guzman, C. K. Thomas, P. Hosur, A. Vishwanath, and D. M. StamperKurn. Ultracold Atoms in a Tunable Optical Kagome Lattice. Phys. Rev. Lett. 108, 045305 (2012). Cited on p. 3.

[38] J. Hubbard. Electron Correlations in Narrow Energy bands. Proc. R. Soc. Lond. 276, 238-257 (1963). Cited on p. 3, 8.

[39] D. Jaksch and P. Zoller. The cold atom Hubbard toolbox. Ann. Phys. 315, 52-79 (2005). Cited on p. 3.

[40] M. Lewenstein, A. Sanpera, V. Ahufinger, B. Damski, A. Sen(De), and U. Sen. Ultracold atomic gases in optical lattices: mimicking condensed matter physics and beyond. Adv. Phys. 56, 243-379 (2007). Cited on p. 3.

[41] T. Esslinger. Fermi-Hubbard physics with atoms in an optical lattice. Annu. Rev. Condens. Matter Phys. 1, 129-152 (2010). Cited on p. 3.

[42] M. P. A. Fisher, P. B. Weichman, G. Grinstein, and D. S. Fisher. Boson localization and the superfluid-insulator transition. Phys. Rev. B 40, 546-570 (1989). Cited on p. 3.

[43] D. Jaksch, C. Bruder, J. I. Cirac, C. W. Gardiner, and P. Zoller. Cold Bosonic Atoms in Optical Lattices. Phys. Rev. Lett. 81, 3108-3111 (1998). Cited on p. 3, 48.

[44] M. Greiner, O. Mandel, T. Esslinger, T. W. Hänsch, and I. Bloch. Quantum phase transition from a superfluid to a Mott insulator in a gas of ultracold atoms. Nature 415, 39-44 (2002). Cited on p. 3, 47, 49, 53, 80, 98.

[45] C. Chin, R. Grimm, P. Julienne, and E. Tiesinga. Feshbach resonances in ultracold gases. Rev. Mod. Phys. 82, 1225-1286 (2010). Cited on p. 3, 148.

[46] N. Gemelke, X. Zhang, C.-L. Hung, and C. Chin. In situ observation of incompressible Mott-insulating domains in ultracold atomic gases. Nature 460, 995-998 (2009). Cited on p. 3.

[47] B. Zimmermann, T. Müller, J. Meineke, T. Esslinger, and H. Moritz. High-resolution imaging of ultracold fermions in microscopically tailored optical potentials. New J. Phys. 13, 043007 (2011). Cited on p. 3.

[48] K. D. Nelson, X. Li, and D. S. Weiss. Imaging single atoms in a three-dimensional array. Nature Phys. 3, 556-560 (2007). Cited on p. 3.

[49] W. S. Bakr, J. I. Gillen, A. Peng, S. Fölling, and M. Greiner. A quantum gas microscope for detecting single atoms in a Hubbard-regime optical lattice. Nature 462, 74-77 (2009). Cited on p. 3, 47. 
[50] J. F. Sherson, C. Weitenberg, M. Endres, M. Cheneau, I. Bloch, and S. Kuhr. Singleatom-resolved fluorescence imaging of an atomic Mott insulator. Nature 467, 68-72 (2010). Cited on p. 3, 47, 83, 149.

[51] T. Gericke, P. Würtz, D. Reitz, T. Langen, and H. Ott. High-resolution scanning electron microscopy of an ultracold quantum gas. Nature Phys. 4, 949-953 (2008). Cited on p. 3.

[52] M. Endres, M. Cheneau, T. Fukuhara, C. Weitenberg, P. Schauß, C. Gross, L. Mazza, M. C. Bañuls, L. Pollet, I. Bloch, and S. Kuhr. Observation of Correlated Particle-Hole Pairs and String Order in Low-Dimensional Mott Insulators. Science 334, 200-203 (2011). Cited on p. 3.

[53] C. Weitenberg, M. Endres, J. F. Sherson, M. Cheneau, P. Schauß, T. Fukuhara, I. Bloch, and S. Kuhr. Single-spin addressing in an atomic Mott insulator. Nature 471, 319-324 (2011). Cited on p. 3.

[54] G. Jotzu, M. Messer, R. Desbuquois, M. Lebrat, T. Uehlinger, D. Greif, and T. Esslinger. Experimental realisation of the topological Haldane model with ultracold fermions. Nature 515, 237-240 (2014). Cited on p. 3, 28, 125, 128.

[55] T. Oka and H. Aoki. Photovoltaic Hall effect in graphene. Phys. Rev. B 79, 081406 (2009). Cited on p. 3.

[56] N. R. Cooper. Rapidly rotating atomic gases. Adv. Phys. 57, 539-616 (2008). Cited on p. 3, 23.

[57] A. L. Fetter. Rotating trapped Bose-Einstein condensates. Rev. Mod. Phys. 81, 647-691 (2009). Cited on p. 3, 23.

[58] J. Dalibard, F. Gerbier, G. Juzeliūnas, and P. Öhberg. Colloquium: Artificial gauge potentials for neutral atoms. Rev. Mod. Phys. 83, 1523-1543 (2011). Cited on p. 3, 23.

[59] N. Goldman, G. Juzeliūnas, P. Öhberg, and I. B. Spielman. Light-induced gauge fields for ultracold atoms. Rep. Prog. Phys. 77, 126401 (2014). Cited on p. 3, 23, 125, 128.

[60] D. Jaksch and P. Zoller. Creation of effective magnetic fields in optical lattices: the Hofstadter butterfly for cold neutral atoms. New J. Phys. 5, 56 (2003). Cited on p. 4, 23,59 .

[61] F. Gerbier and J. Dalibard. Gauge fields for ultracold atoms in optical superlattices. New J. Phys. 12, 033007 (2010). Cited on p. 4, 23, 37, 59.

[62] E. J. Mueller. Artificial electromagnetism for neutral atoms: Escher staircase and Laughlin liquids. Phys. Rev. A 70, 041603 (2004). Cited on p. 4, 59. 
[63] A. R. Kolovsky. Creating artificial magnetic fields for cold atoms by photon-assisted tunneling. Europhys. Lett. 93, 20003 (2011). Cited on p. 4, 59.

[64] C. E. Creffield and F. Sols. Comment on "Creating artificial magnetic fields for cold atoms by photon-assisted tunneling" by Kolovsky A. R. Europhys. Lett. 101, 40001 (2013). Cited on p. 4, 59.

[65] A. Bermudez, T. Schaetz, and D. Porras. Synthetic Gauge Fields for Vibrational Excitations of Trapped Ions. Phys. Rev. Lett. 107, 150501 (2011). Cited on p. 4, 42, 59, 148.

[66] N. Goldman, J. Dalibard, M. Aidelsburger, and N. R. Cooper. Periodically-driven quantum matter: the case of resonant modulations. Phys. Rev. A 91, 033632 (2015). Cited on p. 4, 26, 28, 32, 35, 42, 70, 109, 116, 149.

[67] J. Struck, C. Ölschläger, R. Le Targat, P. Soltan-Panahi, A. Eckardt, M. Lewenstein, P. Windpassinger, and K. Sengstock. Quantum Simulation of Frustrated Classical Magnetism in Triangular Optical Lattices. Science 333, 996-999 (2011). Cited on p. 4, $23,28,59,76$.

[68] M. Aidelsburger, M. Atala, S. Nascimbène, S. Trotzky, Y.-A. Chen, and I. Bloch. Experimental Realization of Strong Effective Magnetic Fields in an Optical Lattice. Phys. Rev. Lett. 107, 255301 (2011). Cited on p. 4, 23, 28, 59, 69, 72, 73, 74, 75, 77, 82, 83, 93, 99, 102, 103, 109, 125.

[69] M. Aidelsburger, M. Atala, S. Nascimbène, S. Trotzky, Y.-A. Chen, and I. Bloch. Experimental realization of strong effective magnetic fields in optical superlattice potentials. Appl. Phys. B 113, 1-11 (2013). Cited on p. 4, 28, 51, 52, 53, 59, 69, 82, 99, 102, 103.

[70] M. Aidelsburger, M. Atala, M. Lohse, J. T. Barreiro, B. Paredes, and I. Bloch. Realization of the Hofstadter Hamiltonian with Ultracold Atoms in Optical Lattices. Phys. Rev. Lett. 111, 185301 (2013). Cited on p. 4, 7, 28, 51, 57, 93, 97, 98, 101, 102, 104, 105, 106, 109, 125.

[71] H. Miyake, G. A. Siviloglou, C. J. Kennedy, W. C. Burton, and W. Ketterle. Realizing the Harper Hamiltonian with Laser-Assisted Tunneling in Optical Lattices. Phys. Rev. Lett. 111, 185302 (2013). Cited on p. 4, 7, 28, 51, 93, 109, 125.

[72] M. Atala, M. Aidelsburger, M. Lohse, J. T. Barreiro, B. Paredes, and I. Bloch. Observation of chiral currents with ultracold atoms in bosonic ladders. Nature Phys. 10, 588-593 (2014). Cited on p. 4, 28, 109, 149.

[73] M. Aidelsburger, M. Lohse, C. Schweizer, M. Atala, J. T. Barreiro, S. Nascimbène, N. R. Cooper, I. Bloch, and N. Goldman. Measuring the Chern number of Hofstadter 
bands with ultracold bosonic atoms. Nature Phys. 11, 162-166 (2015). Cited on p. 4, 5, 20, 28, 42, 57, 109, 111, 112, 113, 116, 126, 129, 132, 135, 137, 138, 139, 140, 141, 142,144 .

[74] M. Y. Azbel. Energy spectrum of a conduction electron in a magnetic field. JETP 19 (1964). Cited on p. 4, 15, 93.

[75] P. G. Harper. Single Band Motion of Conduction Electrons in a Uniform Magnetic Field. Proc. Phys. Soc. A 68, 874 (1955). Cited on p. 4, 15, 93.

[76] D. R. Hofstadter. Energy levels and wave functions of Bloch electrons in rational and irrational magnetic fields. Phys. Rev. B 14, 2239-2249 (1976). Cited on p. 4, 7, 15, 17, 93.

[77] N. Goldman, I. Satija, P. Nikolic, A. Bermudez, M. A. Martin-Delgado, M. Lewenstein, and I. B. Spielman. Realistic Time-Reversal Invariant Topological Insulators with Neutral Atoms. Phys. Rev. Lett. 105, 255302 (2010). Cited on p. 5, 93, 94.

[78] C. R. Dean, L. Wang, P. Maher, C. Forsythe, F. Ghahari, Y. Gao, J. Katoch, M. Ishigami, P. Moon, M. Koshino, T. Taniguchi, K. Watanabe, K. L. Shepard, J. Hone, and P. Kim. Hofstadter's butterfly and the fractal quantum Hall effect in moiré superlattices. Nature 497, 598-602 (2013). Cited on p. 7.

[79] L. A. Ponomarenko, R. V. Gorbachev, G. L. Yu, D. C. Elias, R. Jalil, A. A. Patel, A. Mishchenko, A. S. Mayorov, C. R. Woods, J. R. Wallbank, M. MuchaKruczynski, B. A. Piot, M. Potemski, I. V. Grigorieva, K. S. Novoselov, F. Guinea, V. I. Fal'ko, and A. K. Geim. Cloning of Dirac fermions in graphene superlattices. Nature 497, 594-597 (2013). Cited on p. 7.

[80] B. Hunt, J. D. Sanchez-Yamagishi, A. F. Young, M. Yankowitz, B. J. LeRoy, K. Watanabe, T. Taniguchi, P. Moon, M. Koshino, P. Jarillo-Herrero, and R. C. Ashoori. Massive Dirac Fermions and Hofstadter Butterfly in a van der Waals Heterostructure. Science 340, 1427-1430 (2013). Cited on p. 7.

[81] M. Hafezi, S. Mittal, J. Fan, A. Migdall, and J. M. Taylor. Imaging topological edge states in silicon photonics. Nature Photonics 7, 1001-1005 (2013). Cited on p. 7.

[82] M. C. Rechtsman, J. M. Zeuner, Y. Plotnik, Y. Lumer, D. Podolsky, F. Dreisow, S. Nolte, M. Segev, and A. Szameit. Photonic Floquet topological insulators. Nature 496, 196-200 (2013). Cited on p. 7, 42, 148.

[83] Y. Aharonov and D. Bohm. Significance of Electromagnetic Potentials in the Quantum Theory. Phys. Rev. 115, 485-491 (1959). Cited on p. 7, 9, 23.

[84] N. Ashcroft and N. Mermin. Solid State Physics. Harcourt Brace College Publishers, Fort Worth (1976). Cited on p. 8, 9, 48. 
[85] C. Kittel. Introduction to solid state physics. Wiley, Philadelphia (2004). Cited on p. $8,9,48$.

[86] R. Peierls. Zur Theorie des Diamagnetismus von Leitungselektronen. Z. Phys. 80, 763791 (1933). Cited on p. 8.

[87] E. Brown. Bloch Electrons in a Uniform Magnetic Field. Phys. Rev. 133, A1038-A1044 (1964). Cited on p. 10.

[88] J. Zak. Magnetic Translation Group. Phys. Rev. 134, A1602-A1606 (1964). Cited on p. 10.

[89] J. Zak. Magnetic Translation Group. II. Irreducible Representations. Phys. Rev. 134, A1607-A1611 (1964). Cited on p. 10.

[90] B. A. Bernevig. Topological Insulators and Topological Superconductors. Princeton University Press (2013). Cited on p. 10, 151.

[91] N. Nemec and G. Cuniberti. Hofstadter butterflies of bilayer graphene. Phys. Rev. B 75, 201404 (2007). Cited on p. 17.

[92] R. Bistritzer and A. H. MacDonald. Moiré butterflies in twisted bilayer graphene. Phys. Rev. B 84, 035440 (2011). Cited on p. 17.

[93] T. Hatakeyama and H. Kamimura. Electronic properties of a Penrose tiling lattice in a magnetic field. Solid State Communications 62, 79-83 (1987). Cited on p. 17.

[94] D. Xiao, M.-C. Chang, and Q. Niu. Berry phase effects on electronic properties. Rev. Mod. Phys. 82, 1959-2007 (2010). Cited on p. 19, 20, 23, 128.

[95] A. H. MacDonald. Landau-level subband structure of electrons on a square lattice. Phys. Rev. B 28, 6713-6717 (1983). Cited on p. 20.

[96] G. H. Wannier. A Result Not Dependent on Rationality for Bloch Electrons in a Magnetic Field. Phys. Stat. Sol. B 88, 757-765 (1978). Cited on p. 20.

[97] P. Streda. Quantised Hall effect in a two-dimensional periodic potential. J. Phys. C: Solid State Phys. 15, L1299 (1982). Cited on p. 21.

[98] M. Kohmoto. Zero modes and the quantized Hall conductance of the two-dimensional lattice in a magnetic field. Phys. Rev. B 39, 11943-11949 (1989). Cited on p. 21.

[99] T. Fukui, Y. Hatsugai, and H. Suzuki. Chern Numbers in Discretized Brillouin Zone: Efficient Method of Computing (Spin) Hall Conductances. J. Phys. Soc. Jpn. 74, 16741677 (2005). Cited on p. 21, 22, 143. 
[100] M. R. Matthews, B. P. Anderson, P. C. Haljan, D. S. Hall, C. E. Wieman, and E. A. Cornell. Vortices in a Bose-Einstein Condensate. Phys. Rev. Lett. 83, 2498-2501 (1999). Cited on p. 23.

[101] K. W. Madison, F. Chevy, W. Wohlleben, and J. Dalibard. Vortex Formation in a Stirred Bose-Einstein Condensate. Phys. Rev. Lett. 84, 806-809 (2000). Cited on p. 23.

[102] J. R. Abo-Shaeer, C. Raman, J. M. Vogels, and W. Ketterle. Observation of Vortex Lattices in Bose-Einstein Condensates. Science 292, 476-479 (2001). Cited on p. 23.

[103] Y.-J. Lin, R. L. Compton, A. R. Perry, W. D. Phillips, J. V. Porto, and I. B. Spielman. Bose-Einstein Condensate in a Uniform Light-Induced Vector Potential. Phys. Rev. Lett. 102, 130401 (2009). Cited on p. 23.

[104] Y. J. Lin, R. L. Compton, K. Jimenez-Garcia, J. V. Porto, and I. B. Spielman. Synthetic magnetic fields for ultracold neutral atoms. Nature 462, 628-632 (2009). Cited on p. 23.

[105] M. V. Berry. Quantal Phase Factors Accompanying Adiabatic Changes. Proc. R. Soc. Lond. A 392, 45-57 (1984). Cited on p. 23.

[106] N. R. Cooper. Optical Flux Lattices for Ultracold Atomic Gases. Phys. Rev. Lett. 106, 175301 (2011). Cited on p. 23.

[107] N. R. Cooper and J. Dalibard. Optical flux lattices for two-photon dressed states. Europhys. Lett. 95, 66004 (2011). Cited on p. 23.

[108] K. Jiménez-García, L. J. LeBlanc, R. A. Williams, M. C. Beeler, A. R. Perry, and I. B. Spielman. Peierls Substitution in an Engineered Lattice Potential. Phys. Rev. Lett. 108, 225303 (2012). Cited on p. 23.

[109] J. Struck, C. Ölschläger, M. Weinberg, P. Hauke, J. Simonet, A. Eckardt, M. Lewenstein, K. Sengstock, and P. Windpassinger. Tunable Gauge Potential for Neutral and Spinless Particles in Driven Optical Lattices. Phys. Rev. Lett. 108, 225304 (2012). Cited on p. 23, 28.

[110] W. Magnus. On the exponential solution of differential equations for a linear operator. Comm. Pure and Appl. Math. 7, 649-673 (1954). Cited on p. 24, 30, 32.

[111] M. M. Maricq. Application of average Hamiltonian theory to the NMR of solids. Phys. Rev. B 25, 6622-6632 (1982). Cited on p. 24, 30, 32.

[112] S. Rahav, I. Gilary, and S. Fishman. Effective Hamiltonians for periodically driven systems. Phys. Rev. A 68, 013820 (2003). Cited on p. 24, 26, 27.

[113] J. H. Shirley. Solution of the Schrödinger Equation with a Hamiltonian Periodic in Time. Phys. Rev. 138, B979-B987 (1965). Cited on p. 24, 25. 
[114] T. Fromherz. Floquet states and intersubband absorption in strongly driven double quantum wells. Phys. Rev. B 56, 4772-4777 (1997). Cited on p. 24.

[115] M. Grifoni and P. Hänggi. Driven quantum tunneling. Phys. Rep. 304, 229-354 (1998). Cited on p. 24.

[116] T. Bilitewski and N. R. Cooper. Scattering theory for Floquet-Bloch states. Phys. Rev. A 91, 033601 (2015). Cited on p. 25, 139, 141, 148.

[117] W. R. Salzman. Quantum mechanics of systems periodic in time. Phys. Rev. A 10, 461-465 (1974). Cited on p. 25, 26, 153.

[118] Y. B. Zel'dovich. The quasienergy of a quantum-mechanical system subjected to a periodic action. Sov. Phys. JETP 24, 1006 (1967). Cited on p. 25.

[119] H. Sambe. Steady States and Quasienergies of a Quantum-Mechanical System in an Oscillating Field. Phys. Rev. A 7, 2203-2213 (1973). Cited on p. 25.

[120] F. Gesztesy and H. Mitter. A note on quasi-periodic states. J. Phys. A: Math. Gen. 14, L79-L85 (1981). Cited on p. 25.

[121] N. Goldman and J. Dalibard. Periodically-Driven Quantum Systems: Effective Hamiltonians and Engineered Gauge Fields. Phys. Rev. X 4, 031027 (2014). Cited on p. 26, $27,28,29$.

[122] M. Bukov and A. Polkovnikov. Stroboscopic versus nonstroboscopic dynamics in the Floquet realization of the Harper-Hofstadter Hamiltonian. Phys. Rev. A 90, 043613 (2014). Cited on p. 26, 85.

[123] S. R. Barone, M. A. Narcowich, and F. J. Narcowich. Floquet theory and applications. Phys. Rev. A 15, 1109-1125 (1977). Cited on p. 26, 153.

[124] H. Lignier, C. Sias, D. Ciampini, Y. Singh, A. Zenesini, O. Morsch, and E. Arimondo. Dynamical Control of Matter-Wave Tunneling in Periodic Potentials. Phys. Rev. Lett. 99, 220403 (2007). Cited on p. 28, 34.

[125] J. Struck, M. Weinberg, C. Ölschläger, P. Windpassinger, J. Simonet, K. Sengstock, R. Höppner, P. Hauke, A. Eckhardt, M. Lewenstein, and L. Mathey. Engineering Ising-XY spin-models in a triangular lattice using tunable artificial gauge fields. Nature Phys. 9, 738-743 (2013). Cited on p. 28, 59.

[126] Y.-A. Chen, S. Nascimbène, M. Aidelsburger, M. Atala, S. Trotzky, and I. Bloch. Controlling Correlated Tunneling and Superexchange Interactions with ac-Driven Optical Lattices. Phys. Rev. Lett. 107, 210405 (2011). Cited on p. 28, 51. 
[127] M. Bukov, L. D'Alessio, and A. Polkovnikov. Universal High-Frequency Behavior of Periodically Driven Systems: from Dynamical Stabilization to Floquet Engineering. arXiv:1407.4803 (2014). Cited on p. 30, 31, 33.

[128] P. Hauke, O. Tieleman, A. Celi, C. Olschläger, J. Simonet, J. Struck, M. Weinberg, P. Windpassinger, K. Sengstock, M. Lewenstein, and A. Eckardt. Non-Abelian Gauge Fields and Topological Insulators in Shaken Optical Lattices. Phys. Rev. Lett. 109, 145301 (2012). Cited on p. 30, 31.

[129] W. R. Salzman. An alternative to the magnus expansion in time-dependent perturbation theory. J. Chem. Phys. 82, 822-826 (1985). Cited on p. 32.

[130] A. Eckardt, T. Jinasundera, C. Weiss, and M. Holthaus. Analog of Photon-Assisted Tunneling in a Bose-Einstein Condensate. Phys. Rev. Lett. 95, 200401 (2005). Cited on p. 34 .

[131] N. Teichmann, M. Esmann, and C. Weiss. Fractional photon-assisted tunneling for Bose-Einstein condensates in a double well. Phys. Rev. A 79, 063620 (2009). Cited on p. 34 .

[132] A. Bermudez, T. Schaetz, and D. Porras. Photon-assisted-tunneling toolbox for quantum simulations in ion traps. New J. Phys. 14, 053049 (2012). Cited on p. 42.

[133] M. H. Anderson, J. R. Ensher, M. R. Matthews, C. E. Wieman, and E. A. Cornell. Observation of Bose-Einstein Condensation in a Dilute Atomic Vapor. Science 269, 198201 (1995). Cited on p. 43.

[134] K. B. Davis, M. O. Mewes, M. R. Andrews, N. J. van Druten, D. S. Durfee, D. M. Kurn, and W. Ketterle. Bose-Einstein Condensation in a Gas of Sodium Atoms. Phys. Rev. Lett. 75, 3969-3973 (1995). Cited on p. 43.

[135] M. Greiner. Magnetischer Transfer von Atomen - Ein Weg zur dreifachen Bose-EinsteinKondensation. Diploma thesis, Ludwig-Maximilians-Universität München (2000). Cited on p. 43.

[136] M. Greiner. Ultracold quantum gases in three-dimensional optical lattice potentials. PhD thesis, Ludwig-Maximilians-Universität München (2003). Cited on p. 43, 44.

[137] O. Mandel. Entaglement with quantum gates in an optical lattice. PhD thesis, LudwigMaximilians-Universität München (2005). Cited on p. 43.

[138] A. Widera. Constructing Correlated Spin States with Neutral Atoms in Optical Lattices. PhD thesis, Johannes Gutenberg-Universität Mainz (2007). Cited on p. 43.

[139] S. Fölling. Probing strongly correlated states of ultracold atoms in optical lattices. PhD thesis, Johannes Gutenberg-Universität Mainz (2008). Cited on p. 43, 49. 
[140] M. E. Atala. Measuring Topological Invariants and Chiral Meissner Currents with Ultracold Bosonic Atoms. PhD thesis, Ludwig-Maximilians-Universität München (2014). Cited on p. 43.

[141] M. Aidelsburger. Ultracold atoms in periodically driven optical lattices. Master's thesis, Ludwig-Maximilians-Universität München (2011). Cited on p. 43, 45, 46, 49, 51.

[142] M. Metcalf and P. van der Straten. Laser Cooling and Trapping. Springer, New York (1999). Cited on p. 43, 44.

[143] M. Greiner, I. Bloch, T. W. Hänsch, and T. Esslinger. Magnetic transport of trapped cold atoms over a large distance. Phys. Rev. A 63, 031401 (2001). Cited on p. 43, 45.

[144] A. L. Migdall, J. V. Prodan, W. D. Phillips, T. H. Bergeman, and H. J. Metcalf. First Observation of Magnetically Trapped Neutral Atoms. Phys. Rev. Lett. 54, 2596-2599 (1985). Cited on p. 44.

[145] W. D. Phillips. Nobel Lecture: Laser cooling and trapping of neutral atoms. Rev. Mod. Phys. 70, 721-741 (1998). Cited on p. 44.

[146] D. A. Steck. Rubidium 87 D Line Data. http://steck.us/alkalidata (2010). Cited on p. 44.

[147] W. Petrich, M. H. Anderson, J. R. Ensher, and E. A. Cornell. Behavior of atoms in a compressed magneto-optical trap. J. Opt. Soc. Am. B 11, 1332-1335 (1994). Cited on p. 44.

[148] S. Chu, L. Hollberg, J. E. Bjorkholm, A. Cable, and A. Ashkin. Three-dimensional viscous confinement and cooling of atoms by resonance radiation pressure. Phys. Rev. Lett. 55, 48-51 (1985). Cited on p. 44.

[149] W. D. Phillips and H. Metcalf. Laser Deceleration of an Atomic Beam. Phys. Rev. Lett. 48, 596-599 (1982). Cited on p. 46.

[150] K. Dieckmann, R. J. C. Spreeuw, M. Weidemüller, and J. T. M. Walraven. Twodimensional magneto-optical trap as a source of slow atoms. Phys. Rev. A 58, 3891-3895 (1998). Cited on p. 46.

[151] H. F. Hess. Evaporative cooling of magnetically trapped and compressed spin-polarized hydrogen. Phys. Rev. B 34, 3476-3479 (1986). Cited on p. 46.

[152] N. Masuhara, J. M. Doyle, J. C. Sandberg, D. Kleppner, T. J. Greytak, H. F. Hess, and G. P. Kochanski. Evaporative Cooling of Spin-Polarized Atomic Hydrogen. Phys. Rev. Lett. 61, 935-938 (1988). Cited on p. 46.

[153] W. Ketterle and N. J. van Druten. Evaporative Cooling of Trapped Atoms. Adv. At. Mol. Opt. Phys. 37, 181 (1996). Cited on p. 46. 
[154] W. Petrich, M. H. Anderson, J. R. Ensher, and E. A. Cornell. Stable, Tightly Confining Magnetic Trap for Evaporative Cooling of Neutral Atoms. Phys. Rev. Lett. 74, 3352-3355 (1995). Cited on p. 46.

[155] K. B. Davis, M.-O. Mewes, M. A. Joffe, M. R. Andrews, and W. Ketterle. Evaporative Cooling of Sodium Atoms. Phys. Rev. Lett. 74, 5202-5205 (1995). Cited on p. 46.

[156] C. C. Bradley, C. A. Sackett, J. J. Tollett, and R. G. Hulet. Evidence of Bose-Einstein Condensation in an Atomic Gas with Attractive Interactions. Phys. Rev. Lett. 75, 16871690 (1995). Cited on p. 46.

[157] T. Esslinger, I. Bloch, and T. W. Hänsch. Bose-Einstein condensation in a quadrupoleIoffe-configuration trap. Phys. Rev. A 58, R2664-R2667 (1998). Cited on p. 46.

[158] R. Grimm, M. Weidemüller, and Y. B. Ovchinnikov. Optical Dipole Traps for Neutral Atoms. Adv. At. Mol. Opt. Phys. 42, 95-170 (2000). Cited on p. 46, 47, 66, 94, 110.

[159] C. S. Adams, H. J. Lee, N. Davidson, M. Kasevich, and S. Chu. Evaporative Cooling in a Crossed Dipole Trap. Phys. Rev. Lett. 74, 3577-3580 (1995). Cited on p. 46.

[160] C.-L. Hung, X. Zhang, N. Gemelke, and C. Chin. Accelerating evaporative cooling of atoms into Bose-Einstein condensation in optical traps. Phys. Rev. A 78, 011604 (2008). Cited on p. 46.

[161] T. Gericke, F. Gerbier, A. Widera, S. Fölling, and O. Mandel. Adiabatic loading of a Bose-Einstein condensate in a 3D optical lattice. J. Mod. Opt. 54, 735-743 (2007). Cited on p. 47.

[162] T. Gericke. Charakterisierung stark korrelierter Quantengase in optischen Gittern. Diploma thesis, Johannes Gutenberg-Universität Mainz (2005). Cited on p. 47, 116.

[163] W. S. Bakr, A. Peng, M. E. Tai, R. Ma, J. Simon, J. I. Gillen, S. Fölling, L. Pollet, and M. Greiner. Probing the Superfluid-to-Mott Insulator Transition at the Single-Atom Level. Science 329, 547-550 (2010). Cited on p. 47, 83, 149.

[164] M. Feld. Ultracold bosonic atoms in bichromatic optical superlattices. Diploma thesis, Johannes Gutenberg-Universität Mainz (2007). Cited on p. 49, 51.

[165] W. Ketterle, D. Durfee, and D. Stamper-Kurn. Making, probing and understanding Bose-Einstein condensates. Proc. Int. School of Physics - Enrico Fermi pages 67-176 (1999). Cited on p. 49.

[166] R. Jáuregui, N. Poli, G. Roati, and G. Modugno. Anharmonic parametric excitation in optical lattices. Phys. Rev. A 64, 033403 (2001). Cited on p. 54. 
[167] C. J. Myatt, E. A. Burt, R. W. Ghrist, E. A. Cornell, and C. E. Wieman. Production of Two Overlapping Bose-Einstein Condensates by Sympathetic Cooling. Phys. Rev. Lett. 78, 586-589 (1997). Cited on p. 55.

[168] H. Schmaljohann, M. Erhard, J. Kronjäger, M. Kottke, S. van Staa, L. Cacciapuoti, J. J. Arlt, K. Bongs, and K. Sengstock. Dynamics of F=2 Spinor Bose-Einstein Condensates. Phys. Rev. Lett. 92, 040402 (2004). Cited on p. 55.

[169] M.-S. Chang, C. D. Hamley, M. D. Barrett, J. A. Sauer, K. M. Fortier, W. Zhang, L. You, and M. S. Chapman. Observation of Spinor Dynamics in Optically Trapped ${ }^{87} \mathrm{Rb}$ Bose-Einstein Condensates. Phys. Rev. Lett. 92, 140403 (2004). Cited on p. 55.

[170] S. Nascimbène, Y.-A. Chen, M. Atala, M. Aidelsburger, S. Trotzky, B. Paredes, and I. Bloch. Experimental Realization of Plaquette Resonating Valence-Bond States with Ultracold Atoms in Optical Superlattices. Phys. Rev. Lett. 108, 205301 (2012). Cited on p. 56.

[171] G. Möller and N. R. Cooper. Condensed ground states of frustrated Bose-Hubbard models. Phys. Rev. A 82, 063625 (2010). Cited on p. 59, 63, 65, 76, 77, 78.

[172] L.-K. Lim, C. M. Smith, and A. Hemmerich. Staggered-Vortex Superfluid of Ultracold Bosons in an Optical Lattice. Phys. Rev. Lett. 100, 130402 (2008). Cited on p. 59.

[173] L.-K. Lim, A. Lazarides, A. Hemmerich, and C. M. Smith. Strongly interacting two-dimensional Dirac fermions. Europhys. Lett. 88, 36001 (2009). Cited on p. 59.

[174] L.-K. Lim, A. Hemmerich, and C. M. Smith. Artificial staggered magnetic field for ultracold atoms in optical lattices. Phys. Rev. A 81, 023404 (2010). Cited on p. 59.

[175] L.-K. Lim, A. Lazarides, A. Hemmerich, and C. M. Smith. Competing pairing states for ultracold fermions in optical lattices with an artificial staggered magnetic field. Phys. Rev. A 82, 013616 (2010). Cited on p. 59.

[176] O. Boada, A. Celi, and J. Latorre. Gauge-Away Effect in Cold Gases on Optical Lattices. arXiv:0909.3937 (2009). Cited on p. 63.

[177] O. Boada, A. Celi, J. I. Latorre, and V. Picó. Simulation of gauge transformation on systems of ultracold atoms. New. J. Phys. 12, 113055 (2010). Cited on p. 63.

[178] R. Roth and K. Burnett. Superfluidity and interference pattern of ultracold bosons in optical lattices. Phys. Rev. A 67, 031602 (2003). Cited on p. 65.

[179] W. Zwerger. Mott-Hubbard transition of cold atoms in optical lattices. J. Opt. B: Quantum Semiclass. Opt. 5, 9 (2003). Cited on p. 65.

[180] E. J. Mueller, T.-L. Ho, M. Ueda, and G. Baym. Fragmentation of Bose-Einstein condensates. Phys. Rev. A 74, 033612 (2006). Cited on p. 76. 
[181] P. Nozières. Bose-Einstein Condensation. edited by A. Griffin, D. W. Snoke, S. Stringari, Cambridge University Press (1996). Cited on p. 76.

[182] X. Li and S. Das Sarma. Cyclotron dynamics of interacting bosons in artificial magnetic fields. Phys. Rev. B 89, 224302 (2014). Cited on p. 85.

[183] M. C. Beeler, R. A. Williams, K. Jiménez-García, L. J. LeBlanc, A. R. Perry, and I. B. Spielman. The spin Hall effect in a quantum gas. Nature 498, 201-204 (2013). Cited on p. 93, 106.

[184] K. Osterloh, M. Baig, L. Santos, P. Zoller, and M. Lewenstein. Cold Atoms in NonAbelian Gauge Potentials: From the Hofstadter "Moth" to Lattice Gauge Theory. Phys. Rev. Lett. 95, 010403 (2005). Cited on p. 97.

[185] J. Ruseckas, G. Juzeliūnas, P. Öhberg, and M. Fleischhauer. Non-Abelian Gauge Potentials for Ultracold Atoms with Degenerate Dark States. Phys. Rev. Lett. 95, 010404 (2005). Cited on p. 97.

[186] Y. K. Kato, R. C. Myers, A. C. Gossard, and D. D. Awschalom. Observation of the Spin Hall Effect in Semiconductors. Science 306, 1910-1913 (2004). Cited on p. 106.

[187] J. Wunderlich, B. Kaestner, J. Sinova, and T. Jungwirth. Experimental Observation of the Spin-Hall Effect in a Two-Dimensional Spin-Orbit Coupled Semiconductor System. Phys. Rev. Lett. 94, 047204 (2005). Cited on p. 106.

[188] Jenoptik. Fiber-coupled integrated optical light modulators. http://www.jenoptik.com (2014). Cited on p. 119.

[189] M. Atala, M. Aidelsburger, J. T. Barreiro, D. Abanin, T. Kitagawa, E. Demler, and I. Bloch. Direct measurement of the Zak phase in topological Bloch bands. Nature Phys. 9, 795-800 (2013). Cited on p. 125.

[190] D. A. Abanin, T. Kitagawa, I. Bloch, and E. Demler. Interferometric Approach to Measuring Band Topology in 2D Optical Lattices. Phys. Rev. Lett. 110, 165304 (2013). Cited on p. 125.

[191] L. Duca, T. Li, M. Reitter, I. Bloch, M. Schleier-Smith, and U. Schneider. An Aharonov-Bohm interferometer for determining Bloch band topology. Science 347, 288292 (2015). Cited on p. 125.

[192] R. O. Umucalılar, H. Zhai, and M. Ö. Oktel. Trapped Fermi Gases in Rotating Optical Lattices: Realization and Detection of the Topological Hofstadter Insulator. Phys. Rev. Lett. 100, 070402 (2008). Cited on p. 125.

[193] E. Zhao, N. Bray-Ali, C. J. Williams, I. B. Spielman, and I. I. Satija. Chern numbers hiding in time-of-flight images. Phys. Rev. A 84, 063629 (2011). Cited on p. 125. 
[194] E. Alba, X. Fernandez-Gonzalvo, J. Mur-Petit, J. K. Pachos, and J. J. Garcia-Ripoll. Seeing Topological Order in Time-of-Flight Measurements. Phys. Rev. Lett. 107, 235301 (2011). Cited on p. 125.

[195] H. M. Price and N. R. Cooper. Mapping the Berry curvature from semiclassical dynamics in optical lattices. Phys. Rev. A 85, 033620 (2012). Cited on p. 125, 128, 135.

[196] X.-J. Liu, K. T. Law, T. K. Ng, and P. A. Lee. Detecting Topological Phases in Cold Atoms. Phys. Rev. Lett. 111, 120402 (2013). Cited on p. 125.

[197] L. Wang, A. A. Soluyanov, and M. Troyer. Proposal for Direct Measurement of Topological Invariants in Optical Lattices. Phys. Rev. Lett. 110, 166802 (2013). Cited on p. 125.

[198] N. Goldman, J. Dalibard, A. Dauphin, F. Gerbier, M. Lewenstein, P. Zoller, and I. B. Spielman. Direct imaging of topological edge states in cold-atom systems. Proc. Natl. Acad. Sci. USA 110, 6736-6741 (2013). Cited on p. 125.

[199] A. Dauphin and N. Goldman. Extracting the Chern Number from the Dynamics of a Fermi Gas: Implementing a Quantum Hall Bar for Cold Atoms. Phys. Rev. Lett. 111, 135302 (2013). Cited on p. 125, 128, 129, 135, 136, 137.

[200] M. B. Dahan, E. Peik, J. Reicherl, Y. Castin, and C. Salomon. Bloch Oscillations of Atoms in an Optical Potential. Phys. Rev. Lett. 76, 4508-4511 (1996). Cited on p. 128.

[201] R. V. Gorbachev, J. C. W. Song, G. L. Yu, A. V. Kretinin, F. Withers, Y. Cao, A. Mishchenko, I. V. Grigorieva, K. S. Novoselov, L. S. Levitov, and A. K. Geim. Detecting topological currents in graphene superlattices. Science 346, 448-451 (2014). Cited on p. 128.

[202] I. Carusotto and C. Ciuti. Quantum fluids of light. Rev. Mod. Phys. 85, 299-366 (2013). Cited on p. 148.

[203] C. E. Creffield. Instability and control of a periodically driven Bose-Einstein condensate. Phys. Rev. A 79, 063612 (2009). Cited on p. 148.

[204] C. A. Parra-Murillo, J. Madroñero, and S. Wimberger. Two-band Bose-Hubbard model for many-body resonant tunneling in the Wannier-Stark system. Phys. Rev. A 88, 032119 (2013). Cited on p. 148.

[205] S. Choudhury and E. J. Mueller. Stability of a Floquet Bose-Einstein condensate in a one-dimensional optical lattice. Phys. Rev. A 90, 013621 (2014). Cited on p. 148.

[206] S. Choudhury and E. J. Mueller. Transverse collisional instabilities of a Bose-Einstein condensate in a driven one-dimensional lattice. arXiv:1410.4576 (2014). Cited on p. 148. 
[207] L. D'Alessio and A. Polkovnikov. Many-body energy localization transition in periodically driven systems. Ann. Phys. 333, 19-33 (2013). Cited on p. 148.

[208] M. Langemeyer and M. Holthaus. Energy flow in periodic thermodynamics. Phys. Rev. E 89, 012101 (2014). Cited on p. 148.

[209] A. Lazarides, A. Das, and R. Moessner. Periodic Thermodynamics of Isolated Quantum Systems. Phys. Rev. Lett. 112, 150401 (2014). Cited on p. 148.

[210] C. Sträter and A. Eckardt. Orbital-driven melting of a bosonic Mott insulator. arXiv:1407.7421 (2014). Cited on p. 149.

[211] A. S. Sørensen, E. Demler, and M. D. Lukin. Fractional Quantum Hall States of Atoms in Optical Lattices. Phys. Rev. Lett. 94, 086803 (2005). Cited on p. 149.

[212] M. Hafezi, A. S. Sørensen, E. Demler, and M. D. Lukin. Fractional quantum Hall effect in optical lattices. Phys. Rev. A 76, 023613 (2007). Cited on p. 149.

[213] R. Palmer and D. Jaksch. High-Field Fractional Quantum Hall Effect in Optical Lattices. Phys. Rev. Lett. 96, 180407 (2006). Cited on p. 149.

[214] G. Möller and N. R. Cooper. Composite Fermion Theory for Bosonic Quantum Hall States on Lattices. Phys. Rev. Lett. 103, 105303 (2009). Cited on p. 149.

[215] E. Orignac and T. Giamarchi. Meissner effect in a bosonic ladder. Phys. Rev. B 64, 144515 (2001). Cited on p. 149.

[216] M.-C. Cha and J.-G. Shin. Two peaks in the momentum distribution of bosons in a weakly frustrated two-leg optical ladder. Phys. Rev. A 83, 055602 (2011). Cited on p. 149.

[217] A. Dhar, M. Maji, T. Mishra, R. V. Pai, S. Mukerjee, and A. Paramekanti. BoseHubbard model in a strong effective magnetic field: Emergence of a chiral Mott insulator ground state. Phys. Rev. A 85, 041602 (2012). Cited on p. 149.

[218] A. Dhar, T. Mishra, M. Maji, R. V. Pai, S. Mukerjee, and A. Paramekanti. Chiral Mott insulator with staggered loop currents in the fully frustrated Bose-Hubbard model. Phys. Rev. B 87, 174501 (2013). Cited on p. 149.

[219] A. Petrescu and K. Le Hur. Bosonic Mott Insulator with Meissner Currents. Phys. Rev. Lett. 111, 150601 (2013). Cited on p. 149.

[220] M. Piraud, F. Heidrich-Meisner, I. P. McCulloch, S. Greschner, T. Vekua, and U. Schollwöck. Vortex and Meissner phases of strongly-interacting bosons on a twoleg ladder. arXiv:1409.7016 (2014). Cited on p. 149. 
[221] R. Wei and E. J. Mueller. Theory of bosons in two-leg ladders with large magnetic fields. Phys. Rev. A 89, 063617 (2014). Cited on p. 149.

[222] A. Tokuno and A. Georges. Ground states of a Bose-Hubbard ladder in an artificial magnetic field: field-theoretical approach. New J. Phys. 16, 073005 (2014). Cited on p. 149.

[223] S. Keßler and F. Marquardt. Single-site-resolved measurement of the current statistics in optical lattices. Phys. Rev. A 89, 061601 (2014). Cited on p. 149.

[224] D. Hügel and B. Paredes. Chiral ladders and the edges of quantum Hall insulators. Phys. Rev. A 89, 023619 (2014). Cited on p. 149.

[225] A. Celi, P. Massignan, J. Ruseckas, N. Goldman, I. B. Spielman, G. Juzeliūnas, and M. Lewenstein. Synthetic Gauge Fields in Synthetic Dimensions. Phys. Rev. Lett. 112, 043001 (2014). Cited on p. 149. 


\section{Acknowledgements}

First of all, I would like to thank my supervisor Immanuel Bloch for his great support and assistance during the last years. I learned a lot from him about physics during our discussions and meetings. I deeply appreciate his guidance and also the freedom he gave me in following my own research ideas. He is a great mentor and his enthusiasm and energy were always very inspiring and motivating. I am very grateful that he gave me the opportunity to work in his group which is one of the best in the world. He managed to create a unique scientific environment which consists of knowledgable people in the group as wells as a large network of international scientists which was the foundation for this work. In particular I want to thank all my collaborators Nigel Cooper, Eugene Demler, Jean Dalibard, Nathan Goldman, Belén Paredes, Marin Bukov, Fabian Grusdt, Dmitry Abanin and Takuya Kitagawa without whom all these projects would not have been possible.

Large credit also goes to the whole Boson team, Yu-Ao Chen, Stefan Trotzky, Sylvain Nascimbène, Julio Barreiro, Marcos Atala, Michael Lohse and Christian Schweizer. We spent a lot of time together in the lab measuring but also eating cakes and cookies and having a lot of fun! Many thanks to everybody for the great time and all the nice work, which was only possible due to the incredible work of the whole team. In particular I would like to thank Stefan, Sylvain and Yu-Ao, who where my supervisors during my master's thesis and the first year as a Ph.D. student. They taught me everything I needed to know about the lab and the underlying physics. Many thanks also to Marcos with whom I spent most of the time during my thesis. I enjoyed working with him, he is a great colleague and we had a lot of fun together also outside the lab with watching soccer and having barbecue. We also had two students in our team, Josselin Bernardoff who did an internship in our lab and became a very good friend of mine as well as Philip Zupancic who did his Bachelor's thesis in our group and was so kind to show me around in Boston.

Special thanks also to my office colleagues Francesco Scazza, Christian Hofrichter, Moritz Höfer and the rest of the Ytterbium team, Simon Fölling, Pieter De Groot, Luis Riegger, Diogo Rio Fernandes, for all the nice lunch and coffee discussions. It would have been much less fun without you guys! Our neighbors were always very friendly in borrowing us equipment while we were still building parts of the experiment. The same is true for the other labs in the second floor, the Fermi I and Fermi II lab as well as the labs in 
Garching. Many thanks to the whole group for the good atmosphere and the friendly and helpful environment.

I am also very thankful to Bodo Hecker, Karsten Förster and Oliver Mödl for their great job in designing and repairing all the electronical equipment in the lab. In particular I am grateful to Bodo who is never tired about explaining electronics to everybody who needs advice and is always very friendly and helpful. We also received great support and help in designing and fabricating mechanical equipment from Michael Pruscha, Markus Böhm and Anton Mayer at the Max-Planck-Institute of Quantum Optics in Garching and the workshop at the faculty of Physics at the LMU led by Jürgen Aust und Thomas Großhauser.

Many thanks for all the organization to Zorah Hauck, Ildiko Kecskesi, Marianne Kargl and Kristina Schuldt. They were always very kind in helping us with all the issues regarding the filling of a variety of different forms also non-work related ones and took care about the arrangement of several group events which were always great.

I acknowledge financial support from the Max-Planck Institute of Quantum Optics during the first few months of my Ph.D. and the Deutsche Telekom Stiftung for supporting me during three years of my doctoral thesis. The Deutsche Telekom Stiftung did not only provide financial support but established a mentoring program. In my case I had the pleasure of being introduced to Dr. Hans-Jürgen Schinzler from the Munich Reinsurance Company with whom I had many interesting conversations and who helped me and is still helping me with his advice and assistance.

Thanks to my family and all my friends for their support and for distracting me from time to time from the work in the lab! And last but not least a warm thank you to Josef Kaftanci for being always there for me and cheering me up whenever I was depressed and lost and for reminding me that there is also a life outside the lab! 